

\section{EDITORIAL BOARD}

ANTONIO CARCATERRA ERIC A. CARLEN

FRANCESCO DELL'ISOLA

RAFFAELE ESPOSITO

ALBERT FANNJIANG

Gilles A. FrancFort

PiERANGElo MARCATI

JEAN-JACQUES MARIGO

PETER A. MARKOWICH MARTIN OSTOJA-STARZEWSKI

PIERRE SEPPECHER

DAVID J. STEIGMANN

PAUl STEINMANN

PierRe M. Suquet

\section{MANAGING EDITORS}

MICOL AMAR

CORRADO LATTANZIO

ANGELA MADEO

MARTIN OSTOJA-STARZEWSKI

\section{ADVISORY BOARD}

ADNAN AKAY

Holm AltenbaCH

MICOL AMAR

HARM ASKES

TEODOR ATANACKOVIĆ

VICTOR BERDICHEVSKY

GuY BouchitTÉ

ANDREA BRAIDES

ROBERTO CAMASSA

MAURO CARFORE

ERIC DARVE

FELIX DARVE

ANNA DE MASI

Gianpietro Del Piero

EMMANUELE Di BENEDETTO

BERNOLD FIEDLER

IRENE M. GAMBA

SERGEY GAVRILYUK

TIMOTHY J. HEALEY

DOMINIQUE JEULIN

ROGER E. KHAYAT

CORRADO LATTANZIO

ROBERT P. LIPTON

ANGELO LUONGO

ANGEla MadeO

JUAN J. MANFREDI

CARLO MARCHIORO

GÉrard A. MAUGin

ROBERTO NATALINI

PATRIZIO NEFF

ANDREY PIATNITSKI

ERrico Presutti

MARIO PUlVIRENTI

LuCiO RuSSO

Miguel A. F. SANJUAN

Patrick SElvadurai

ALEXANDER P. SEYRANIAN

MIROSLAV ŠILHAVÝ

GUIDO SWEERS

ANTOINETTE TORDESILLAS

LEV TRUSKINOVSKY

JUAN J. L. VELÁZQUEZ

VINCENZO VESPRI

ANGELO VULPIANI msp.org/memocs

Università di Roma “La Sapienza”, Italia

Rutgers University, USA

(CO-CHAIR) Università di Roma "La Sapienza", Italia

(TREASURER) Università dell'Aquila, Italia

University of California at Davis, USA

(CO-CHAIR) Université Paris-Nord, France

Università dell' Aquila, Italy

École Polytechnique, France

DAMTP Cambridge, UK, and University of Vienna, Austria

(CHAIR MANAGING EDITOR) Univ. of Illinois at Urbana-Champaign, USA

Université du Sud Toulon-Var, France

University of California at Berkeley, USA

Universität Erlangen-Nürnberg, Germany

LMA CNRS Marseille, France

Università di Roma “La Sapienza”, Italia

Università dell' Aquila, Italy

Université de Lyon-INSA (Institut National des Sciences Appliquées), France (CHAIR MANAGING EDITOR) Univ. of Illinois at Urbana-Champaign, USA

Carnegie Mellon University, USA, and Bilkent University, Turkey

Otto-von-Guericke-Universität Magdeburg, Germany

Università di Roma "La Sapienza”, Italia

University of Sheffield, UK

University of Novi Sad, Serbia

Wayne State University, USA

Université du Sud Toulon-Var, France

Università di Roma Tor Vergata, Italia

University of North Carolina at Chapel Hill, USA

Università di Pavia, Italia

Stanford University, USA

Institut Polytechnique de Grenoble, France

Università dell'Aquila, Italia

Università di Ferrara and International Research Center MEMOCS, Italia

Vanderbilt University, USA

Freie Universität Berlin, Germany

University of Texas at Austin, USA

Université Aix-Marseille, France

Cornell University, USA

École des Mines, France

University of Western Ontario, Canada

Università dell' Aquila, Italy

Louisiana State University, USA

Università dell' Aquila, Italia

Université de Lyon-INSA (Institut National des Sciences Appliquées), France

University of Pittsburgh, USA

Università di Roma "La Sapienza", Italia

Université Paris VI, France

Istituto per le Applicazioni del Calcolo "M. Picone", Italy

Universität Duisburg-Essen, Germany

Narvik University College, Norway, Russia

Università di Roma Tor Vergata, Italy

Università di Roma "La Sapienza", Italia

Università di Roma “Tor Vergata", Italia

Universidad Rey Juan Carlos, Madrid, Spain

McGill University, Canada

Moscow State Lomonosov University, Russia

Academy of Sciences of the Czech Republic

Universität zu Köln, Germany

University of Melbourne, Australia

École Polytechnique, France

Bonn University, Germany

Università di Firenze, Italia

Università di Roma La Sapienza, Italia

MEMOCS (ISSN 2325-3444 electronic, 2326-7186 printed) is a journal of the International Research Center for the Mathematics and Mechanics of Complex Systems at the Università dell'Aquila, Italy.

Cover image: "Tangle" by @ John Horigan; produced using the Context Free program (contextfreeart.org).

\section{PUBLISHED BY}

mathematical sciences publishers

nonprofit scientific publishing

http://msp.org/

(C) 2013 Mathematical Sciences Publishers 


\title{
SELF-ORGANIZED STOCHASTIC TIPPING IN SLOW-FAST DYNAMICAL SYSTEMS
}

\author{
MATHiAs LinKeRHAND AND Claudius GROS
}

\begin{abstract}
Polyhomeostatic adaption occurs when evolving systems try to achieve a target distribution function for certain dynamical parameters, a generalization of the notion of homeostasis. Here we consider a single rate-encoding leaky integrator neuron model driven by white noise, adapting slowly its internal parameters, threshold and gain, in order to achieve a given target distribution for its timeaveraged firing rate. For the case of sparse encoding, when the target firing-rate distribution is bimodal, we observe the occurrence of spontaneous quasiperiodic adaptive oscillations resulting from fast transition between two quasistationary attractors. We interpret this behavior as self-organized stochastic tipping, with noise driving the escape from the quasistationary attractors.
\end{abstract}

\section{Introduction}

Self-regulation plays an important role in biological and technical systems. Homeostatically regulated steady states are a precondition to life, examples being the concentration of blood glucose controlled by insulin [Plum et al. 2006] and glucagon, the $\mathrm{pH}$ value of blood [Schaefer 1961; Tresguerres et al. 2010], and body temperature [Charkoudian 2003], which are all autoregulated in order to maintain stable conditions. Further examples are the concentration of ions, proteins, and transmitters in the brain; their respective levels are all self regulated [Marder and Goaillard 2006]. Furthermore, homeostasis is implemented and can be found in technical systems, for example in microrobotic swarms [Kernbach and Kernbach 2011]. Adaption typically introduces a slow time scale into the dynamical system [Gros 2010b], a process also called metalearning, a central notion in the context of neuromodulation [Doya 2002] and emotional control [Gros 2010a]. The resulting dynamical system then has both fast and slow variables and critical transitions in the form of tipping processes may occur [Kuehn 2011].

Classical homeostasis involves the regulation of a scalar quantity such as body temperature. More complex forms of homeostasis are also important in the realm

PACS2010: $05.10 . \mathrm{Gg}, 05.40 . \mathrm{Ca}, 05.45 .-\mathrm{a}, 05.45 . \mathrm{Tp}, 05.65 .+\mathrm{b}$.

Keywords: stochastic tipping, complex systems, chaos, intrinsic adaption, slow-fast, metalearning. 
of life. For example, an animal may want to achieve a certain time-averaged distribution of behaviors, like foraging, resting, and engaging socially, over the course of several days. This kind of adaptive behavior has been termed polyhomeostasis [Marković and Gros 2010; 2012]. It occurs when a dynamical system tries to achieve, via the continuous adaption of slow variables, a given target distribution for the time-averaged activity of a subset of fast variables. Polyhomeostatically adapting systems are typically slow-fast dynamical systems and their dynamical behavior can tip spontaneously from one state into another. Polyhomeostasis may therefore result in nontrivial dynamical phenomena. Tipping transitions from laminar flow to intermittent chaotic bursts of activities have been observed for networks of rate-encoding and polyhomeostatic adapting neurons [Marković and Gros 2010; 2012].

Tipping transitions can occur both in adaptive and in driven systems. Potential tipping scenarios are currently discussed intensively in the context of climate research [Lenton et al. 2008; Ashwin et al. 2012]. They may be related to a slow driving of external parameters [Baer et al. 1989], to noisy input inducing a stochastic escape from a local attractor [Gammaitoni et al. 1998; McDonnell and Abbott 2009], or through a dynamical effect when the rate of change of a control parameter reaches a certain threshold [Ashwin et al. 2012].

Here we study the phenomenon of self-organized tipping for a polyhomeostatic adapting system driven by a steady-state stochastic input. We examine a previously proposed model [Stemmler and Koch 1999; Triesch 2005] for regulating the firingrate distribution of individual neurons based on information-theoretical principles. This type of model has been studied previously for the case of discrete time systems and unimodal target firing-rate distributions [Marković and Gros 2010; 2012]. Here we examine the case of continuous time and bimodal target distribution functions, corresponding to sparse coding. For bimodal firing-rate distributions the neural activity tends to switch in between states close to minimal and maximal activity. Similar bimodal activity states are also observed in many other systems, for example, dynamical gene regulation networks [Davidson and Erwin 2006]. We find that bimodal target distributions may lead to self-organized bistability within a certain range of parameters.

We consider a single leaky integrator neuron with noisy input and a sigmoidal transfer function having two degrees of freedom. To achieve a special behaviorhere the temporal output distribution of the firing rate - we use polyhomeostasis to change the intrinsic parameters which are directly influencing the mapping of the membrane potential to the firing rate in order to obtain a specific output distribution. We derive these parameter-changing rules using stochastic adaption and show that two degrees of freedom already result in a good behavior approximation, for most of the parameters studied. For bimodal adaption target distributions we observe 
self-organized and quasiperiodic stochastic tipping in between two quasistationary attractors resulting from competing adaption gradients.

\section{Model}

Biological neurons integrate incoming signals and emit an axon potential, a spike, whenever the membrane potential has reached a certain threshold. The membrane potential then returns, after a short refractory period, rapidly to its resting value. This behavior can be captured using spiking integrate-and-fire neural models [Burkitt 2006]. In many circumstances the firing rate, the number of spikes per unit time, is important and rate-encoding neural models can be used [Borst and Theunissen 1999]. Here we consider a single rate-encoding leaky integrator driven by white noise $\xi(t)$,

$$
\dot{x}(t)=-\Gamma x(t)+\xi(t), \quad\left\langle\xi(t) \xi\left(t^{\prime}\right)\right\rangle=Q \delta\left(t-t^{\prime}\right),
$$

where $x>0$ is the membrane potential and $\Gamma>0$ the relaxation rate. The firing rate $y(t) \in[0,1]$ is a nonlinear function of the membrane potential $x(t)$, which we have selected as

$$
y(t)=g(x(t)), \quad g(x)=\frac{1}{1+e^{-a(x-b)}},
$$

where $a>0$ is the gain and $b$ is the threshold. The polynomial transfer function (2) maps the membrane potential $x \in[-\infty, \infty]$ to the normalized firing rate $y \in$ $[0,1]$ which approaches zero and unity for small and large membrane potentials, respectively, compare Figure 1. The slope of $g(x)$ is $a / 4$ at the threshold $b$.

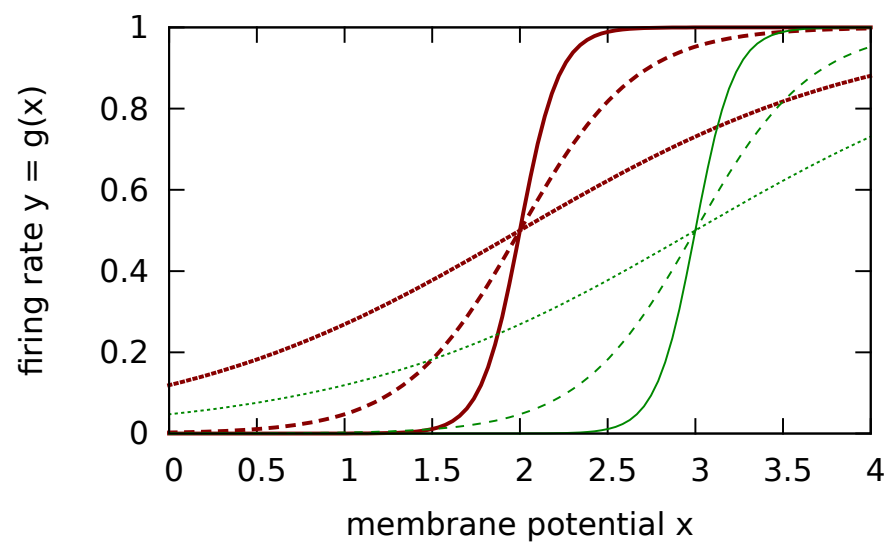

Figure 1. The transfer function $g(x)$ of (2) for thresholds $b=2$ (red lines) and $b=3$ (green lines) and various gains $a: 1$ (dotted), 3 (dashed), and 9 (solid). 
Usually the intrinsic parameters of the transfer function (2), $a$ and $b$, are taken as given by some a priori considerations. Here we will consider them to be slow variables, $a=a(t)$ and $b=b(t)$, adapting slowly such that a target dynamical behavior is approached on the average for the firing rate $y(t)$. The stochastic driving $\xi(t) \in\left[\Xi_{1}, \Xi_{2}\right]$ in (1) is simulated through white noise plateaus: The values are generated according to a uniform probability distribution (white noise), but they remain constant for short time intervals on the order of unity. The membrane potential averages the input driving noise, due to the leak rate $\Gamma$ in (1), its distribution function $\rho(x)$ having a mean $\mu_{\rho} \approx\left(\Xi_{1}+\Xi_{2}\right) /(2 \Gamma)$ and variance $\sigma_{\rho}^{2} \approx\left(\Xi_{2}-\Xi_{1}\right) /(2 \Gamma)$.

2.1. Polyhomeostatic adaption. The firing-rate statistic is given by

$$
p(z)=\frac{1}{T} \int_{t_{0}}^{t_{0}+T} \delta(z-y(t)) \mathrm{d} t, \quad \int_{0}^{1} p(z) \mathrm{d} z=1,
$$

where the length $T$ of the sliding observation window is substantially larger than the relaxation rate $1 / \Gamma$. The firing-rate distribution $p(z)$ is an important quantity characterizing the information processing capability of biological and artificial neurons. No information is encoded for a constant firing rate, the next value is always exactly the same as before, so no new information is transferred. One may assume that a certain distribution $q(y)$ of firing rates may constitute an optimal working regime. Possible functional dependencies for $q(y)$ can be derived by information-theoretical considerations, for example, maximizing information entropy, as discussed further below.

Considering a given target firing-rate distribution $q(y)$, the closeness of the actual firing-rate distribution $p(y)$ is measured by the Kullback-Leibler divergence $D_{\mathrm{KL}}$, their relative entropy [Gros 2010b]:

$$
D_{\mathrm{KL}}(p, q)=\int \mathrm{d} y p(y) \ln \frac{p(y)}{q(y)}, \quad D_{\mathrm{KL}}(p, q) \geq 0 .
$$

The Kullback-Leibler divergence is positive definite and vanishes only when the two distributions coincide. The KL-divergence is generically not symmetric but becomes symmetric in the limiting case of similar distributions $p$ and $q$, becoming equivalent in this limit to the $\chi^{2}$ test [Gros 2010b]. Our aim is now to rewrite (4) as an integral over the membrane potential $x$, using

$$
p(y) \mathrm{d} y=\rho(x) \mathrm{d} x, \quad p(y)=\frac{\rho(x)}{\mathrm{d} y / \mathrm{d} x},
$$

where $\rho(x)$ is the membrane potential distribution. Using $y=g(x)$ and (4) and (5), we obtain

$$
\frac{\partial D_{\mathrm{KL}}}{\partial \theta}=\int \mathrm{d} x \rho(x)\left[-\frac{1}{g^{\prime}} \frac{\partial g^{\prime}}{\partial \theta}-\frac{q^{\prime}}{q} \frac{\partial g}{\partial \theta}\right] \equiv \int \mathrm{d} x \rho(x) \frac{\partial d}{\partial \theta}
$$


for the derivative of the Kullback-Leibler divergence with respect to the intrinsic parameters $\theta=a, b$ of the transfer function $g(x)$; see (2).

We consider now the case in which the system does not dispose of prior information about the distribution of input stimuli and the thereby resulting distribution of membrane potential $\rho(x)$. The best strategy to minimize the Kullback-Leibler divergence is then to minimize the individual terms of the integral (6) through the stochastic adaption rules [Triesch 2005; Marković and Gros 2010]

$$
\frac{\mathrm{d} \theta}{\mathrm{d} t}=-\epsilon_{\theta} \frac{\partial d}{\partial \theta}, \quad \theta=a, b,
$$

for the intrinsic parameters of the transfer function $g(x)$, where the $\epsilon_{\theta}$ are appropriate small adaption rates.

2.2. Target firing-rate distribution. In order to evaluate (7), respectively (6), we need to specify the target firing-rate distribution $q(y)$. For this purpose we use information-theoretical considerations.

Given a continuous probability distribution function $q$ its Shannon entropy $H(q)$ can be defined as

$$
H(q)=-\int \mathrm{d} y q(y) \ln q(y) .
$$

Among all the real-valued distributions with specified mean $\mu$ and standard deviation $\sigma$ the Gaussian distribution [Gros 2010b]

$$
q(y) \propto \exp \left(-\frac{(y-\mu)^{2}}{2 \sigma^{2}}\right) \propto \exp \left(\lambda_{1} y+\lambda_{2} y^{2}\right)
$$

has maximal information entropy, with $\mu=-\lambda_{1} /\left(2 \lambda_{2}\right)$ and $2 \sigma^{2}=-1 / \lambda_{2}$, which is easily obtained using variational calculus:

$$
0=\delta\left[H(q)+\lambda_{1} \int \mathrm{d} y y q(y)+\lambda_{2} \int \mathrm{d} y y^{2} q(y)\right],
$$

where $-\lambda_{1}$ and $-\lambda_{2}$ are the respective Lagrange parameters. In Figure 2 examples for $q(y)$ are illustrated for several values of $\lambda_{1}$ and $\lambda_{2}$. The support of the target firing rates is compact, $y \in[0,1]$, and both negative and positive $\lambda_{1}$ and $\lambda_{2}$ can be considered. The normalization factor $\int_{0}^{1} \mathrm{~d} y q(y)$ cancels out in (6), since only ratios are involved.

For positive $\lambda_{2}>0$ and $\lambda_{1} \approx-\lambda_{2}$ one obtains bimodal target distributions. This is an interesting case, since sparse coding, which is realized when only a minority of neurons in a given network is active, and a majority is inactive [Olshausen and Field 2004], is characterized by a skewed bimodal distribution. 


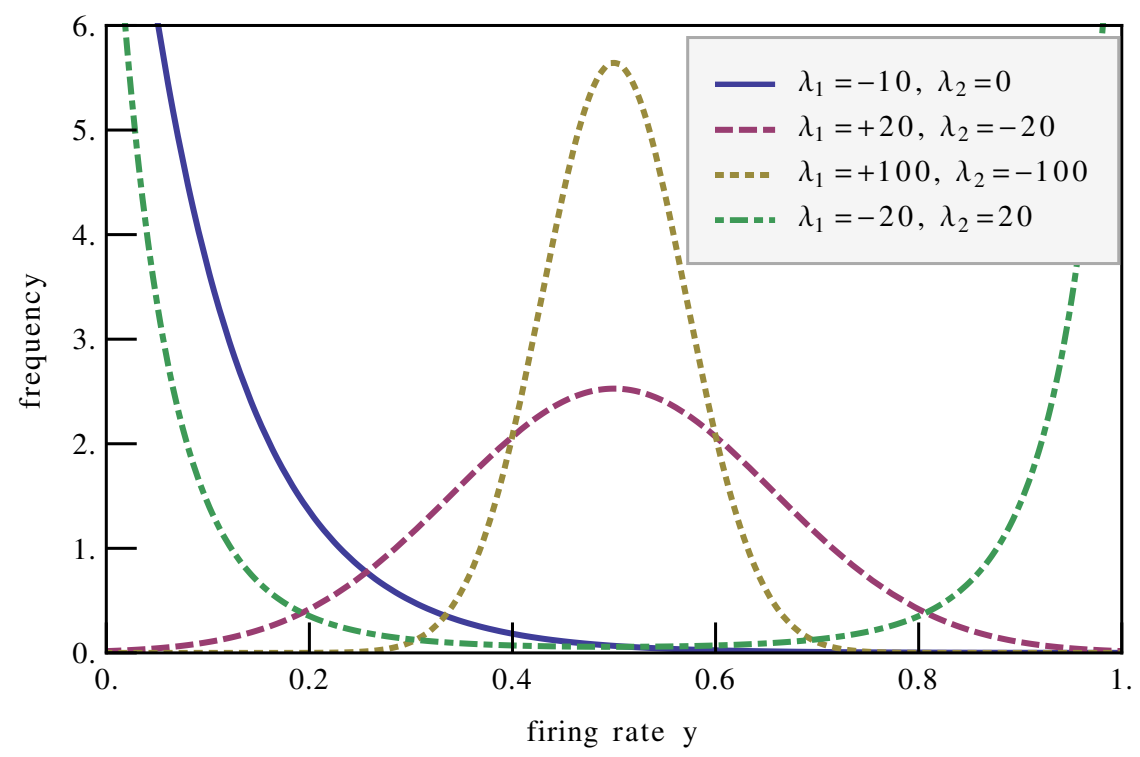

Figure 2. Target distribution $q(y)$, see (9), with some selected parameters $\lambda_{1}$ and $\lambda_{2}$. The target firing-rate distributions are bimodal for $\lambda_{2}>0$.

2.3. Stochastic adaption rules. From (9) and (2) we find the relations

$$
\frac{q^{\prime}(y)}{q(y)}=\lambda_{1}+2 \lambda_{2} y, \quad \frac{\partial g}{\partial x}=a g(1-g),
$$

and

$$
\frac{\partial g}{\partial a}=(x-b) g(1-g), \quad \frac{\partial g}{\partial b}=-a g(1-g),
$$

which we can use to evaluate the stochastic adaption rules (7) as

$$
\frac{\mathrm{d} a}{\mathrm{~d} t}=\epsilon_{a}\left[\frac{1}{a}+(x-b)\left[1-2 y+\left(\lambda_{1}+2 \lambda_{2} y\right)(1-y) y\right]\right]
$$

and

$$
\frac{\mathrm{d} b}{\mathrm{~d} t}=\epsilon_{b}\left[-a\left(1-2 y+\left(\lambda_{1}+2 \lambda_{2} y\right)(1-y) y\right)\right] .
$$

These two adaption rules will lead to an adaption of the time-averaged firing-rate distribution $p(y)$ towards the target distribution $q(x)$ whenever the adaption timescales $1 / \epsilon_{\theta}$ are substantially larger than the time constants of the neural dynamics, which in turn are determined by the time scale of the incoming stimuli and by the leak-rate $\Gamma$ in (1).

The transfer function $g(x)$ contains only two free parameters, the gain $a$ and the threshold $b$. Perfect adaption $p(y) \equiv q(y)$, for all $y \in[0,1]$, can hence not 
be expected. The system tries to minimize the Kullback-Leibler divergence by adapting the available degrees of freedom, which are just two in our case.

2.4. Numerical method. Equations (1), (10), and (11) form a set of first-order differential equations with respect to time. We solve them numerically using the Euler method with one evaluation per time step. We confirmed that this method is sufficiently accurate using the fourth-order Runge-Kutta method [Press et al. 2007]. The random white noise $\xi(t)$ is generated through a pseudorandom number generator with a uniform distribution. The values for the leak $\Gamma$, the time step $\Delta t$, and the learning rates $\epsilon_{a}$ and $\epsilon_{b}$ are shown in the corresponding figures.

\section{Results}

We performed a series of simulations with the aim of studying two issues. Polyhomeostatic adaption has been studied previously for the case of discrete time systems [Triesch 2005; Marković and Gros 2010]; here we examine the case of continuous time. The case of a bimodal target distribution is, in addition, highly interesting, as it confronts the system with a dilemma. The transfer function $g(x)$, compare Figure 1, is strictly monotonic. The distribution of the membrane potential $\rho(x)$ is hence unimodal. There is no easy way for the adapting neuron to achieve, as a steady-state time-average, a bimodal output firing-rate distribution $p(y)$. The question then is whether the system will find a way out of this dilemma through spontaneous behavioral changes.

3.1. Target distribution approximation. For most simulations we used, if not stated otherwise, $\Gamma=1$ for the leak rate and $\Delta t=10^{-1}$ for the integration time step. A typical time series is given in Figure 3. Note that the adaption of the intrinsic parameters $a$ and $b$ takes place on a slower time scale than that of the primary dynamic variables, $x$ and $y$, as typical for a slow-fast dynamical system.

Applying moderate to small learning rates $\epsilon_{a}=\epsilon_{b} \lesssim 0.01$ the neuron's firing rate $y$ approximates various types of target distributions $q$ quite well. In Figure 4 the achieved and the respective target firing-rate distributions are compared. The respective relative entropies are well minimized and presented in Table 1. Strictly speaking the stochastic adaption rules (10) and (11) are equivalent to approximating the firing-rate statistic (3), which is a time-averaged quantity, towards the target distribution function $q(y)$ only in the limit of very small adaption rates, $\epsilon_{a}$ and $\epsilon_{b}$. Small but finite values for the adaption rates, as used in our simulations, correspond to a trailing averaging procedure over a limited time interval, and the value of Kullback-Leibler divergence achieved hence depends weakly on the actual values used for the learning rates.

For very high learning rates, $\epsilon_{b} \gg 0.1$, the threshold $b$ follows the membrane potential $x$ nearly instantaneously, and both variables become highly correlated. 

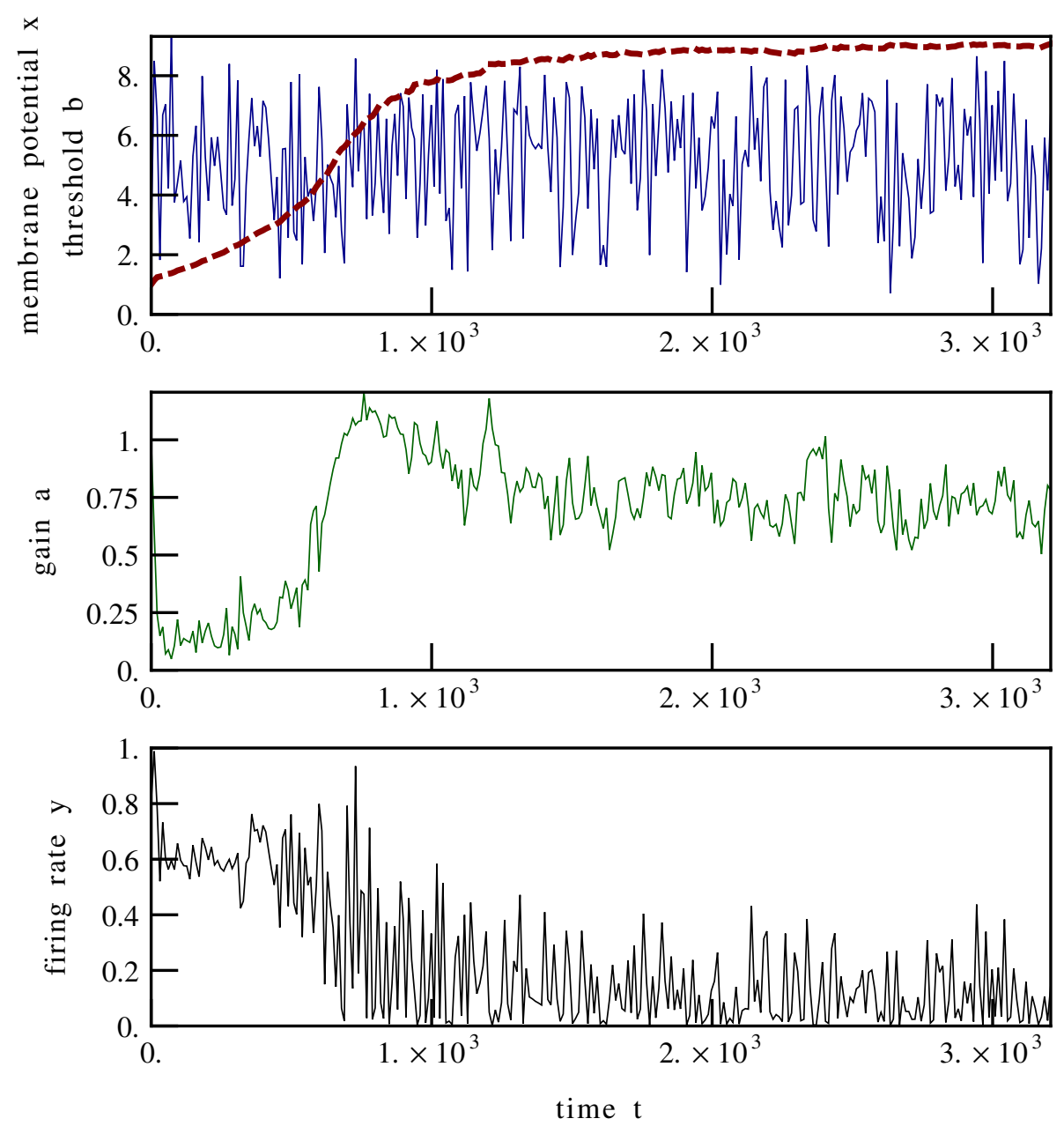

Figure 3. Typical time series for a unimodal target distribution $q(y)$ with $\lambda_{1}=-10, \lambda_{2}=0$, compare Figure 2. Plotted are the membrane potential $x$ (solid blue line, upper panel), the threshold $b$ (dashed red line, upper panel), the gain $a$ (solid green line, middle panel) and the firing rate $y$ (solid black line, lower panel). $\Delta t=10^{-1}, \epsilon_{a}=\epsilon_{b}=10^{-2}$, and $\Gamma=1$.

Therefore the firing-rate distribution $p$ cannot approximate the target distribution $q$ anymore. In fact, the resulting Kullback-Leibler divergence is then very high. The tipping in dynamic behavior as a function of adaption rate amplitude is typical for a rate-induced tipping transition [Ashwin et al. 2012].

3.2. Gain-threshold phase diagram. Due to the sigmoidal shape of the transfer function, several target distributions lead to specific fingerprints in the gain-threshold 

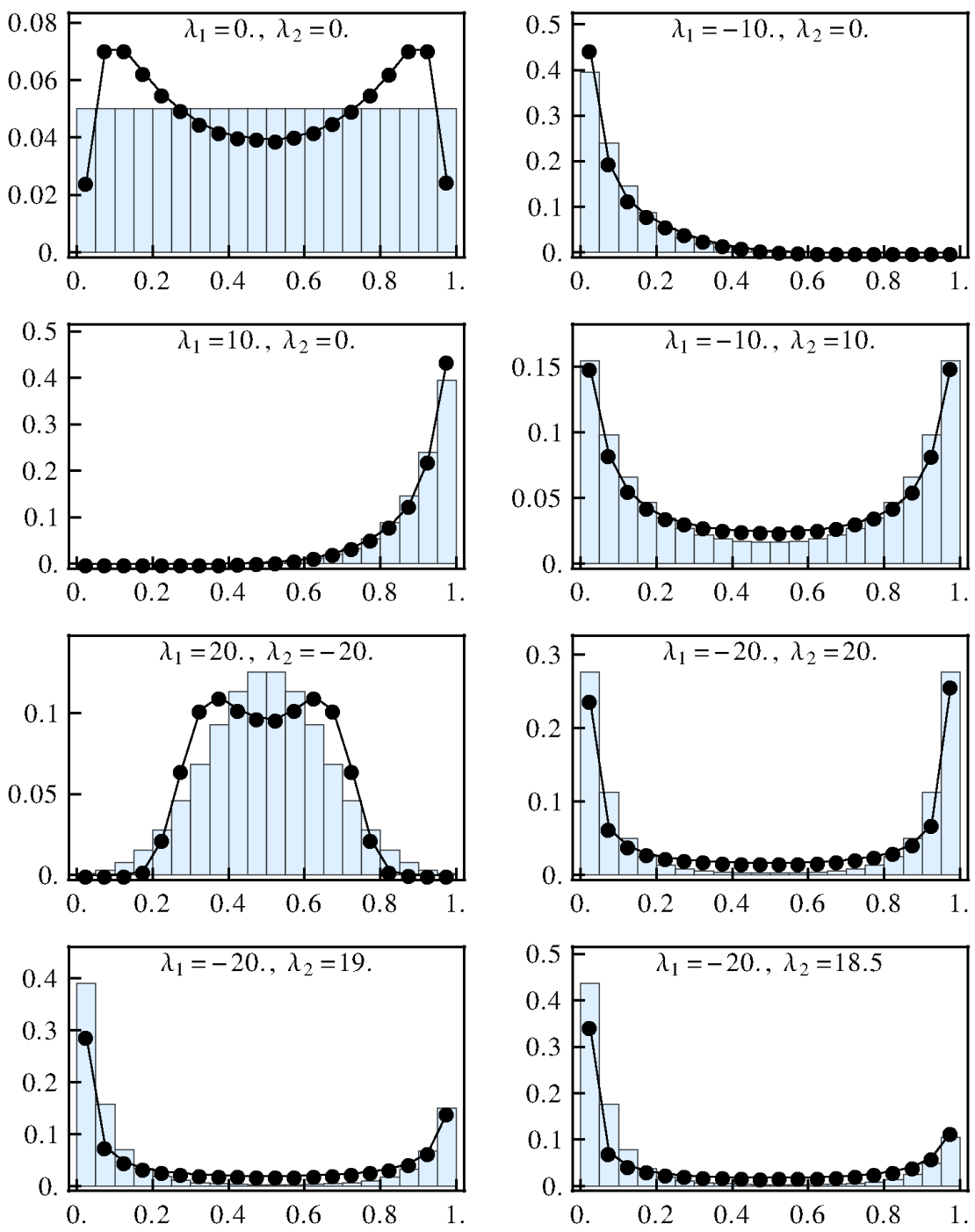

Figure 4. Target distribution $q$ (bars) versus achieved distribution $p$ (points) for different distributions. In each diagram $\lambda_{1}$ and $\lambda_{2}$ are given, while $\Delta t=10^{-1}, t_{\max }=10^{8}, \epsilon_{a}=\epsilon_{b}=10^{-2}, \Gamma=1$, and $\Xi=[0,10]$.

phase diagram which we present in Figure 5. The threshold, for example, for a left (right)-dominant target distribution is high (low) and is therefore sensitive to the mean $\mu=-\lambda_{1} /\left(2 \lambda_{2}\right)$ of $q(y)$. Small gains $a$ result in quite flat transfer functions $g(x)$, compare Figure 1, mapping the membrane potentials to similar firing rates $y$. High gains in $a$ discriminate, relative to the threshold $b$, on the other side 


\begin{tabular}{|rrlc|}
\hline$\lambda_{1}$ & $\lambda_{2}$ & Shape & $D_{K L}$ \\
0 & 0 & Uniform & 0.043 \\
-10 & 0 & Left-dominant & 0.034 \\
+10 & 0 & Right-dominant & 0.028 \\
-10 & +10 & Left/right-dominant & 0.018 \\
+20 & -20 & Hill & 0.076 \\
-20 & +20 & Left/right, symmetric & 0.175 \\
-20 & +19 & Left/right, left-skewed & 0.244 \\
-20 & +18.5 & Left/right, left-skewed & 0.283 \\
\hline
\end{tabular}

Table 1. The relative entropies $D_{K L}$ (4) of various target distributions (see Figure 2) compared to the corresponding achieved distribution, compare Figure 4.

between high and low membrane potentials. The gain is therefore smaller for hillshaped and flat target distributions, as compared to the left and right-dominant target distributions (for example, $\lambda_{1}=-20, \lambda_{2}=+20$ ) for which intermediate values are suppressed.

Left (right)-dominant target distributions (compare Table 1) correspond directly to high (low) transfer function thresholds. Uniform, hill, and other not unilateral dominant target distributions lead to intermediate transfer function thresholds with a wide variety of the transfer function gains. For symmetrical target distributions from hill-shaped to diametrical-shaped there is a transition from low to high gains.

3.3. Self-organized stochastic escape. While the left- and right-dominant unimodal target distributions are easily approximated due to the sigmoidal shape of the transfer function, the bimodal left and right-dominant target distributions put the system in a dilemma: Since intermediate values are to be suppressed the transfer function gain $a$ cannot be too small. Because of this there exist at least two quasistationary fixed points, one for the left part and one for the right part of the distribution.

For zero or small learning rates $\epsilon_{a}=\epsilon_{b} \approx 0$ the system is trapped in a single local fixed point. Only the left or right part of the target distribution is then approximated, and the Kullback-Leibler divergence is not well minimized.

Increasing the learning rates $\epsilon_{a}=\epsilon_{b}$ allows the system to escape stochastically from the respective local fixed points: the transfer function threshold $b$ conquers the local gradient and moves to the other fixed point and back (compare Figure 6). In the long-term observation the system therefore approximates both the left and the right part of the target distribution and hence minimizes the relative entropy, compare Table 2. These tipping transitions between the two quasistationary fixed points are illustrated in Figure 7, which shows a typical time series for a skewed 

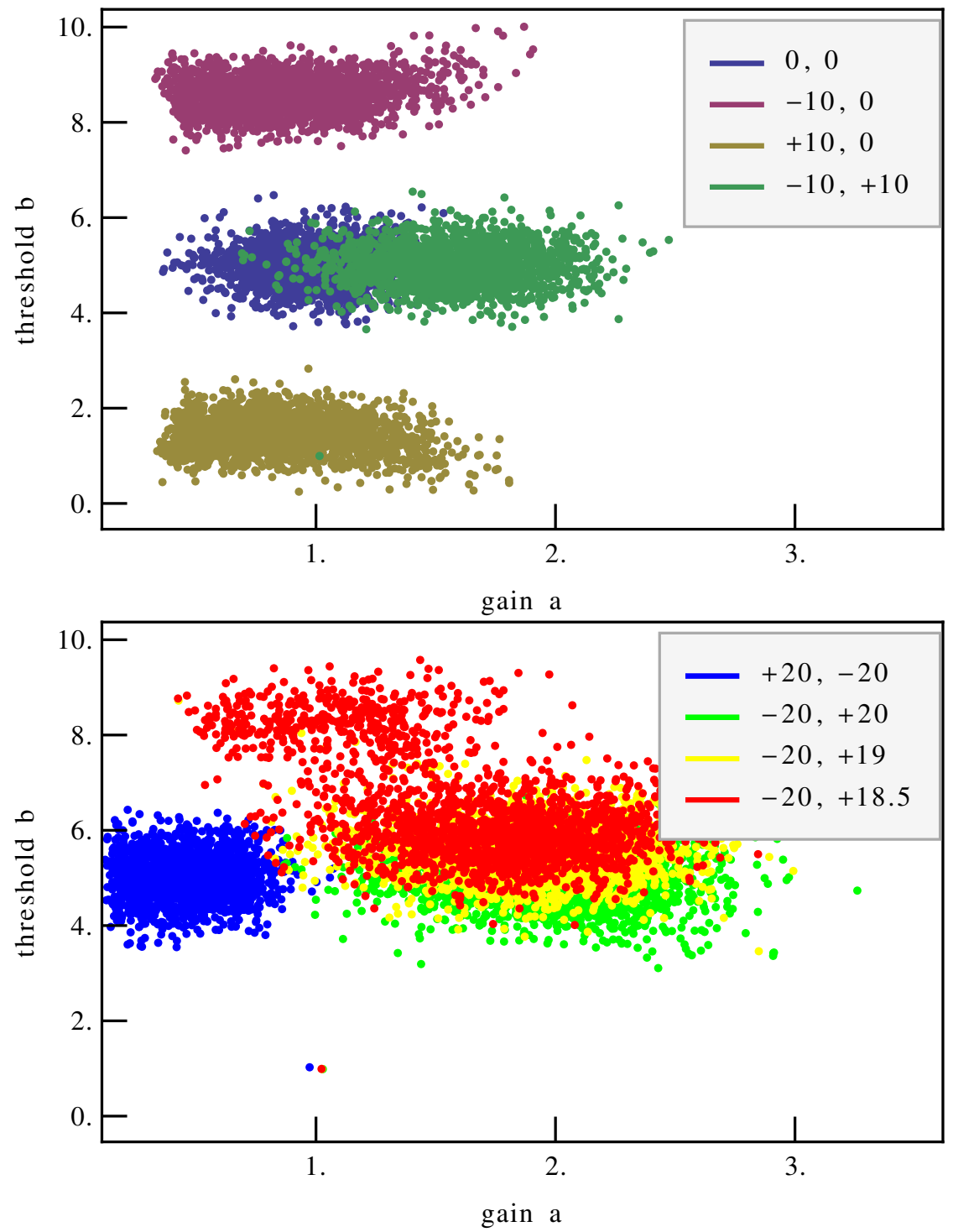

Figure 5. Phase diagram: Plotted are the gain $a(t)$ and the threshold $b(t)$ of the transfer function for various target distributions $\left(\lambda_{1}\right.$ and $\lambda_{2}$ given in the legend). The respective target and achieved firing-rate distributions are given in Figure 4.

target distribution. Note that there are two fixed points for the gain and threshold and a direct correspondence to the periods of high and low firing rates $y(t)$.

Very low learning rates $\epsilon_{a}$ and $\epsilon_{b}$ lead to deep and large basins of attraction for the respective fixed points, while on the other hand high learning rates result in the 


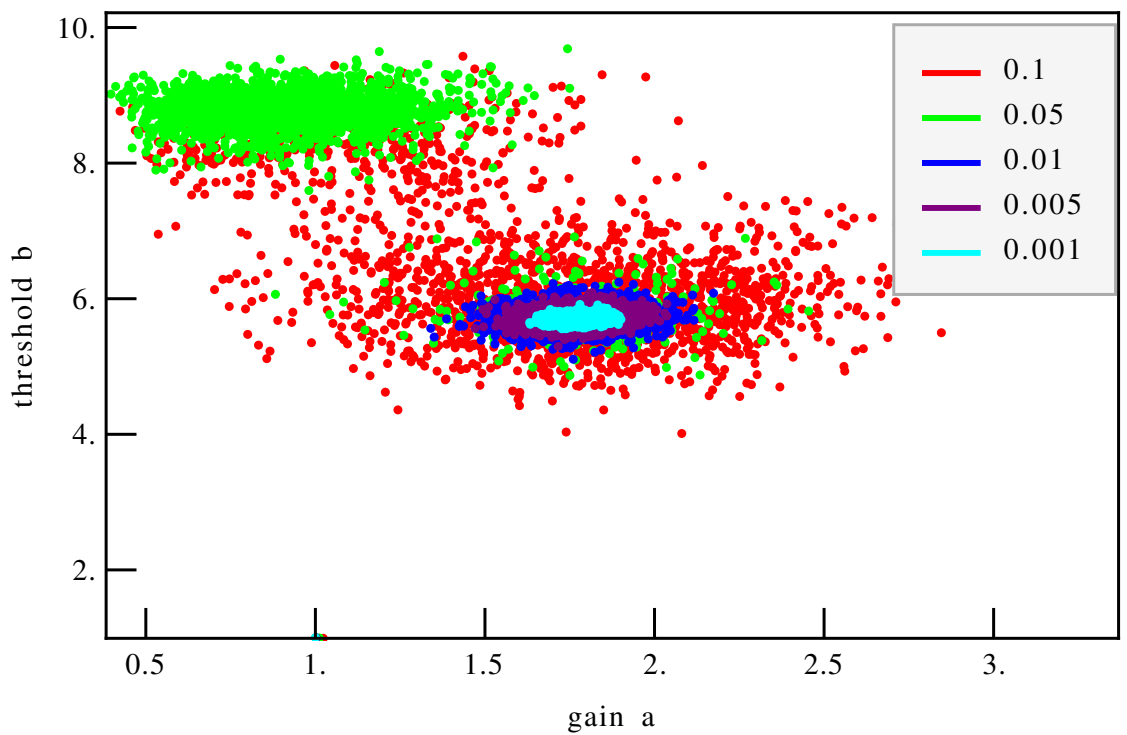

Figure 6. Stochastic escape: Phase diagram of the transfer function gain versus transfer function threshold for a convex leftskewed target distribution with various learning rates $\left(\epsilon_{a}=\epsilon_{b}\right.$ given in the legend), with $\Delta t=10^{-1}, \Gamma=1, \lambda_{1}=-20$, and $\lambda_{2}=18.5$.

close following of the threshold to the membrane potential which prohibits reaching the target distribution. This mechanism is reminiscent of the case of Langevin dynamics in a double-well potential [Hanggi 1986], where a stochastically driven particle may switch forth and back between two local minima [Gros 2010b]. The switching time is controlled for the double-well problem by the Kramers escape rate, which depends exponentially on the potential barrier height. It is difficult to formulate a quantitative mapping to the double-well problem. The local attractors visible in Figures 6 and 7, and the effective barriers in between them, are selforganized structures. Note that the strength $Q$ of the noise term (1) is constant and influences the transition rate only weakly, due to the continuous adaption of the transfer function, via (10) and (11), to the average strength of the stochastic driving.

\begin{tabular}{|lccccccc|}
\hline$\epsilon_{a}=\epsilon_{b}$ & $10^{-5}$ & $10^{-4}$ & $10^{-3}$ & $5 \cdot 10^{-3}$ & $10^{-2}$ & $5 \cdot 10^{-2}$ & $10^{-1}$ \\
$D_{K L}$ & 0.306 & 0.295 & 0.293 & 0.289 & 0.283 & 0.154 & 0.109 \\
\hline
\end{tabular}

Table 2. Relative entropies $D_{K L}$ (4) for the left-skewed target distribution $\left(\lambda_{1}=-20, \lambda_{2}=18.5\right)$ relative to the achieved distribution for various learning rates $\epsilon_{a}$ and $\epsilon_{b}$, compare Figure 6. 

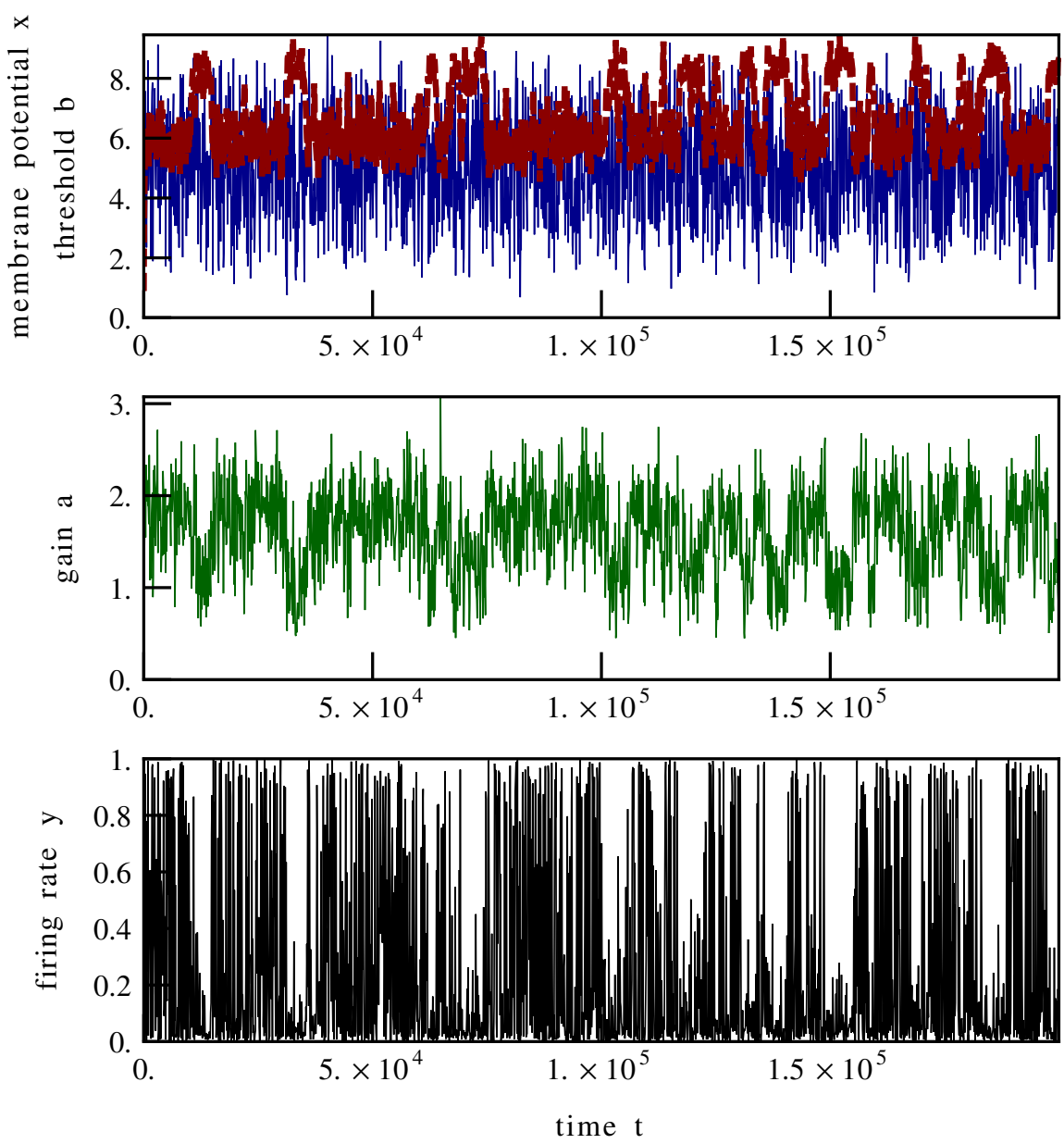

Figure 7. Time series of the membrane potential $x$ (top, blue line), threshold $b$ (top, red line), gain $a$ (middle, green line), and firing rate $y$ (bottom, black line), with $\Delta t=10^{-1}, \epsilon_{a}=\epsilon_{b}=10^{-1}, \Gamma=1$, $\lambda_{1}=-20$, and $\lambda_{2}=18.5$.

\section{Discussion}

We showed that polyhomeostatic adaption of a continuous-time leaky integrator leads to the desired firing-rate distributions. We also run further simulations using white noise and Gaussian noise input and replace the transfer function by other qualitatively different (but still sigmoidal) functions, see the Appendix. It turns out that the polyhomeostatic adaption as well as the self-organized stochastic escape are quite robust principles. However, the quality of the approximation (as seen by visual overlapping) and the value of the Kullback-Leibler divergence depend on the learning rates, and also on the input distribution and the input's strength. 
The stochastic tipping as a function of adaption rates has a close relation to the phenomenon of stochastic escape. The strength of the driving input noise is constant, but its influence is averaged out for very low adaption rates. Stochastic escape from one local attractor to another is not possible. The stochasticity of the input becomes relevant for intermediate values of adaption rates and stochastic transitions between the two quasistationary attractors are most frequent. Finally, for very large adaption rates, the system tips into another dynamical state, tracking the stochastic input signal nearly instantaneously. This sequence of behaviors is self organized and can be reached from any initial state.

\section{Appendix: Polynomial transfer function}

The polyhomeostatic adaption of the system does not change qualitatively by replacing the transfer function $g$. Instead it turns out that the system is robust against changing the transfer function as long as it remains sigmoidal. We also applied a transfer function

$$
g(x)=\frac{(x / b)^{a b}}{(x / b)^{a b}+1},
$$

with a polynomial decay to $g(0)=0$, which limits the membrane potential $x \geq 0$ to be nonnegative. It turns out that the shape of the target distribution $q$ is also well approximated using this transfer function. Stochastic escape from one fixed point to another and back can be observed in addition, since two fixed points are necessary for some target distributions.

The transfer function has an inflection point for exponents $a b>0$; it is absent for $a b<1$, compare Figure 8. The transfer function $g$ behaves as

$$
g(x) \approx \begin{cases}(x / b)^{a b}, & x \ll b, \\ \frac{1}{2}+\frac{1}{4} a(x-b), & x \approx b, \\ 1-(b / x)^{a b}, & x \gg b .\end{cases}
$$

The slope is $a / 4$ which approaches zero and unity for small and large membrane potentials respectively.

From (A.1) we find the relations

$$
\begin{gathered}
\frac{\partial g}{\partial x}=(1-g) g \frac{a b}{x} \\
\frac{\partial g}{\partial a}=(1-g) g b \ln \left(\frac{x}{b}\right), \quad \frac{\partial g}{\partial b}=(1-g) g a\left[\ln \left(\frac{x}{b}\right)-1\right],
\end{gathered}
$$

which we can use to evaluate the stochastic adaption rules (7) as

$$
\frac{\mathrm{d} a}{\mathrm{~d} t}=\epsilon_{a}\left[\frac{1}{a}-b \ln (x / b)\left[1-2 y+\left(\lambda_{1}+2 \lambda_{2} y\right)(1-y) y\right]\right]
$$




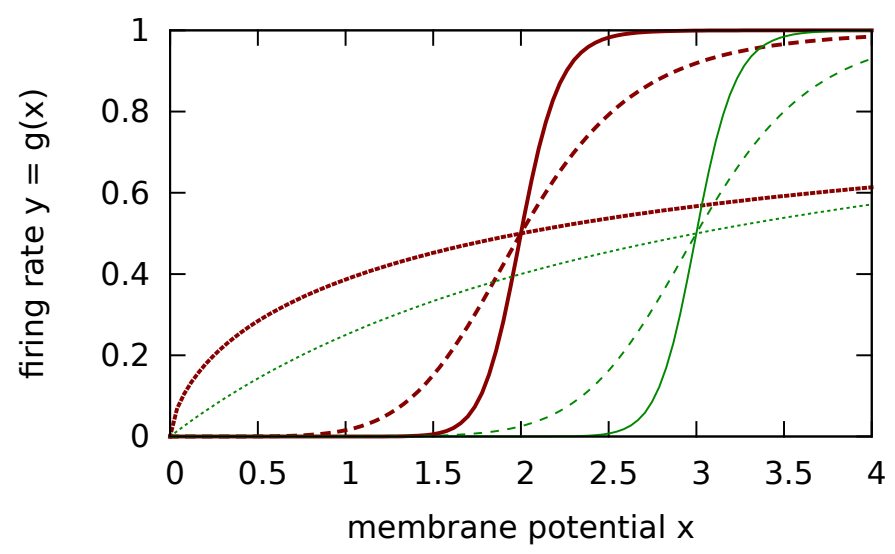

Figure 8. The transfer function $g(x)$, see (A.1), for thresholds $b=2$ (red lines) and $b=3$ (green lines) and various gains $a: 1 / 3$ (dotted), 3 (dashed), and 9 (solid). No inflection point is present for exponents $a b<1$.

and

$$
\frac{\mathrm{d} b}{\mathrm{~d} t}=\epsilon_{b}\left[\frac{1}{b}-a[\ln (x / b)-1]\left[1-2 y+\left(\lambda_{1}+2 \lambda_{2} y\right)(1-y) y\right]\right] .
$$

Applying this transfer function $g$ it turns out that the target distribution is well approximated also in this case, even though the membrane potential is restricted to nonnegative numbers. Table 3 lists the well-minimized Kullback-Leibler divergences for several target distributions.

\begin{tabular}{|rrlc|}
\hline$\lambda_{1}$ & $\lambda_{2}$ & Shape & $D_{K L}$ \\
0 & 0 & Uniform & 0.060131 \\
-10 & 0 & Left-dominant & 0.069351 \\
+10 & 0 & Right-dominant & 0.114578 \\
-10 & +10 & Left/right-dominant & 0.051811 \\
+20 & -20 & Hill & 0.148098 \\
-20 & +20 & Left/right, symmetric & 0.189217 \\
-20 & +19 & Left/right, left-skewed & 0.063934 \\
-20 & +18.5 & Left/right, left-skewed & 0.261215 \\
\hline
\end{tabular}

Table 3. Relative entropies of various target distributions compared to the corresponding achieved distribution $\left(\epsilon_{a}=\epsilon_{b}=10^{-2}\right.$, bins $=100)$. 
We conclude that the stochastic adaption rules are therefore generic and qualitatively independent on the concrete realization of the transfer function. However, quantitatively the resulting relative entropies depend on the choice of the transfer function which also influences the optimal adaption rates $\epsilon_{a}$ and $\epsilon_{b}$.

A.1 Self-organized stochastic escape. For the nonsymmetric convex target distribution $\left(\lambda_{1}=-20, \lambda_{2}=19\right)$ there are two fixed points. Since the target distribution cannot be well approximated by only one fixed point the system escapes stochastically from one to the other and back within a certain period, compare with Figures 9 and 10 . For small learning rates $\epsilon_{a}=\epsilon_{b} \lesssim 0.01$ the system is trapped in only one fixed point. The relative entropy therefore is not well minimized.

For intermediate learning rates $0.01 \lesssim \epsilon_{a}=\epsilon_{b} \lesssim 0.04$ the perturbation is high enough to stochastically escape from that fixed point and approach another one. Figures 9 and 10 show a typical time series for this tipping. This has also an effect on the relative entropy which is therefore even smaller than without stochastic escape (see Table 4).

For high learning rates $\epsilon_{a}=\epsilon_{b} \gtrsim 0.05$ the system's behavior changes: the transfer function is close to a Heaviside step function and the threshold follows the membrane potential quickly. In that state the achieved distribution is not close

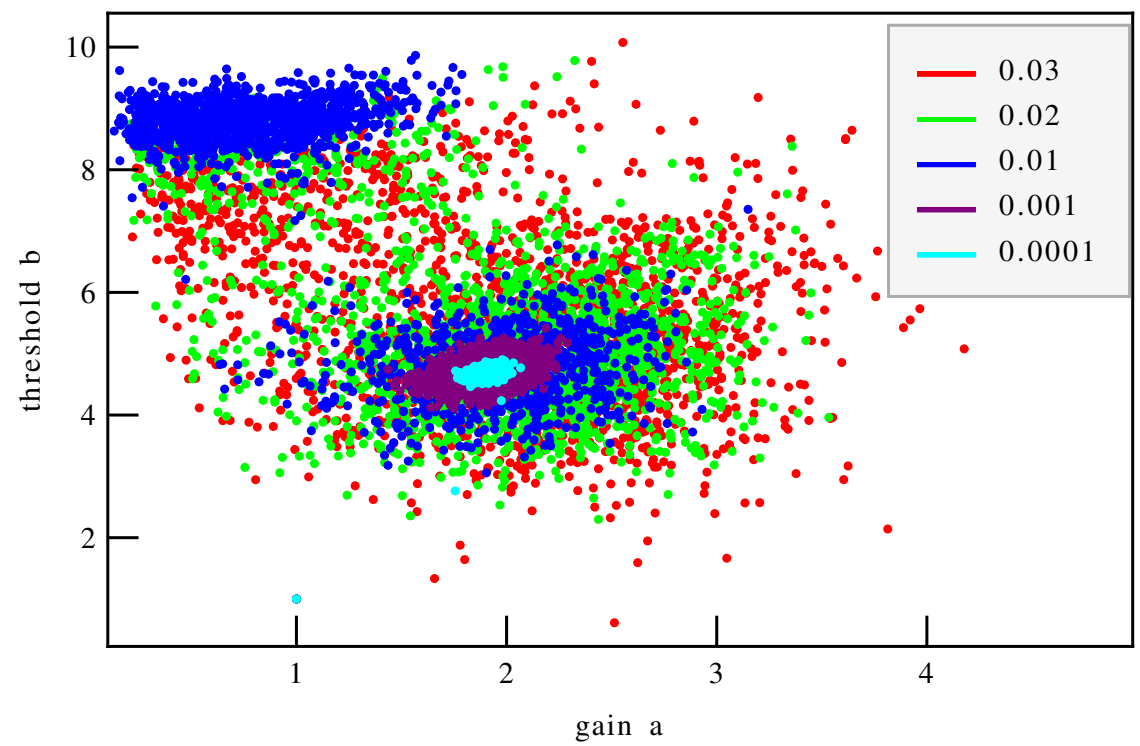

Figure 9. Stochastic escape: Phase diagram of the transfer function gain versus transfer function threshold for a convex leftskewed target distribution with various learning rates $\left(\epsilon_{a}\right.$ and $\epsilon_{b}$ given in the legend), with $\Delta t=10^{-2}, \Gamma=0.1, \lambda_{1}=-20$, and $\lambda_{2}=19$. 

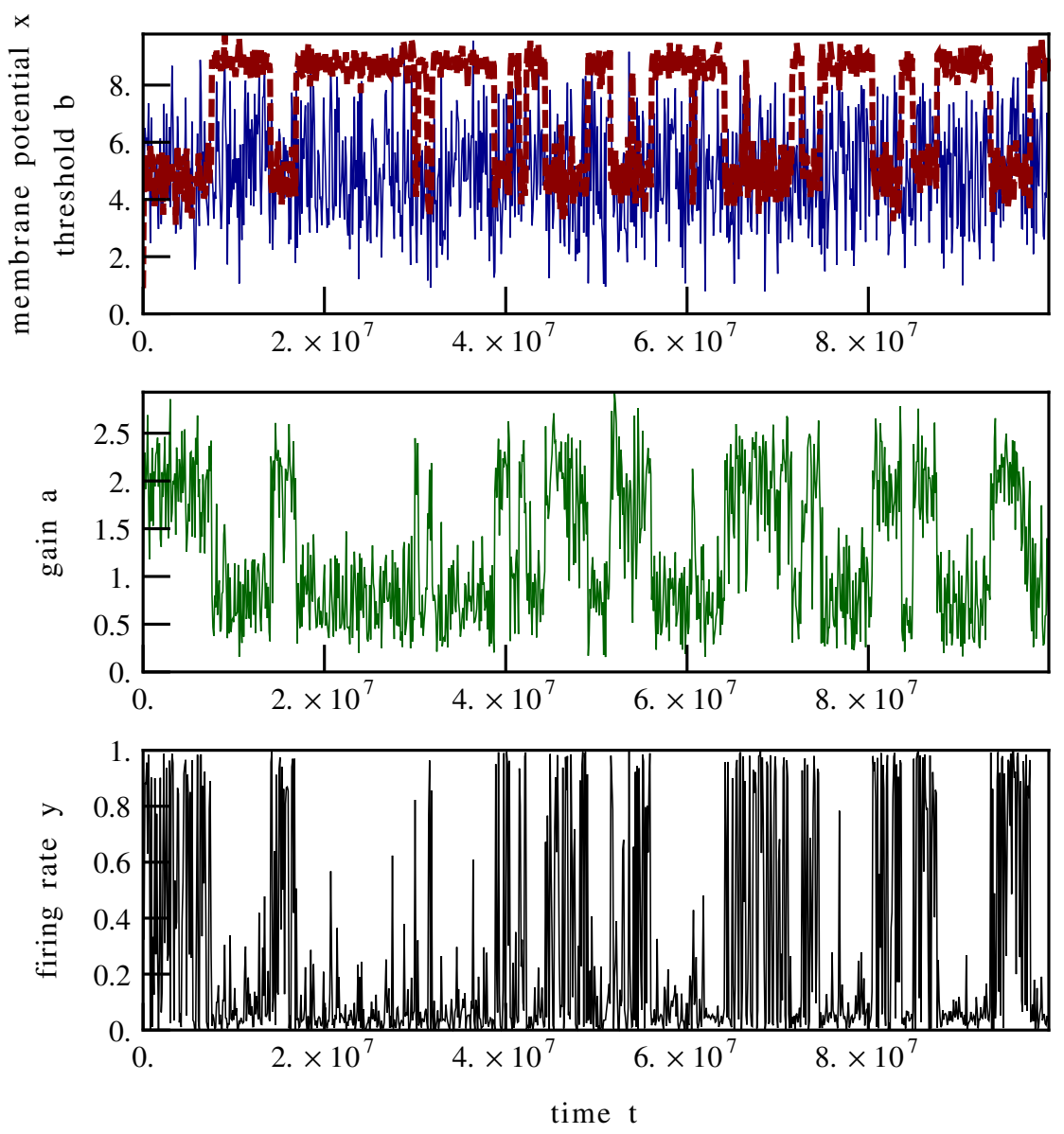

Figure 10. Time series: membrane potential $x$, transfer function threshold $b$ (dashed), transfer function gain $a$, and firing rate $y$, with $\Delta t=10^{-1}, \epsilon_{a}=\epsilon_{b}=10^{-2}, \Gamma=0.1, \lambda_{1}=-20$, and $\lambda_{2}=19$.

to the target distribution, therefore the relative entropy is not minimized anymore (see Table 4).

\begin{tabular}{|lccccccc|}
\hline$\epsilon_{a}=\epsilon_{b}$ & $10^{-4}$ & $10^{-3}$ & 0.01 & 0.03 & 0.04 & 0.05 & 0.06 \\
$D_{K L}$ & 0.376 & 0.368 & 0.064 & 0.043 & 0.017 & 1.892 & 1.591 \\
\hline
\end{tabular}

Table 4. Relative entropies of the left-skewed target distribution $\left(\lambda_{1}=-20, \lambda_{2}=19\right)$ compared to the achieved distribution for various learning rates $\epsilon_{a}$ and $\epsilon_{b}$. Note that the Kullback-Leibler divergence is not minimized for $\epsilon_{b} \gtrsim 0.05$ due to the fast correlation of the membrane potential and the transfer function threshold. 


\section{References}

[Ashwin et al. 2012] P. Ashwin, S. Wieczorek, R. Vitolo, and P. Cox, "Tipping points in open systems: bifurcation, noise-induced and rate-dependent examples in the climate system", Phil. Trans. R. Soc. A 370:1962 (2012), 1166-1184.

[Baer et al. 1989] S. M. Baer, T. Erneux, and J. Rinzel, "The slow passage through a Hopf bifurcation: delay, memory effects, and resonance", SIAM J. Appl. Math. 49:1 (1989), 55-71.

[Borst and Theunissen 1999] A. Borst and F. E. Theunissen, "Information theory and neural coding", Nat. Neurosci. 2:11 (1999), 947-957.

[Burkitt 2006] A. N. Burkitt, "A review of the integrate-and-fire neuron model, I: Homogeneous synaptic input”, Biol. Cybern. 95:1 (2006), 1-19.

[Charkoudian 2003] N. Charkoudian, "Skin blood flow in adult human thermoregulation: how it works, when it does not, and why", Mayo Clin. Proc. 78:5 (2003), 603-612.

[Davidson and Erwin 2006] E. H. Davidson and D. H. Erwin, "Gene regulatory networks and the evolution of animal body plans", Science 311:5762 (2006), 796-800.

[Doya 2002] K. Doya, "Metalearning and neuromodulation”, Neural Netw. 15:4-6 (2002), 495-506.

[Gammaitoni et al. 1998] L. Gammaitoni, P. Hänggi, P. Jung, and F. Marchesoni, "Stochastic resonance", Rev. Mod. Phys. 70:1 (1998), 223-288.

[Gros 2010a] C. Gros, "Cognition and emotion: perspectives of a closing gap", Cogn. Comput. 2:2 (2010), 78-85. arXiv 1002.3035

[Gros 2010b] C. Gros, Complex and adaptive dynamical systems: a primer, 2nd ed., Springer, Berlin, 2010.

[Hanggi 1986] P. Hanggi, "Escape from a metastable state", J. Stat. Phys. 42:1-2 (1986), 105-148.

[Kernbach and Kernbach 2011] S. Kernbach and O. Kernbach, "Collective energy homeostasis in a large-scale microrobotic swarm”, Robot. Auton. Sys. 59:12 (2011), 1090-1101.

[Kuehn 2011] C. Kuehn, "A mathematical framework for critical transitions: bifurcations, fast-slow systems and stochastic dynamics", Physica D 240:12 (2011), 1020-1035. arXiv 1101.2899

[Lenton et al. 2008] T. M. Lenton, H. Held, E. Kriegler, J. W. Hall, W. Lucht, S. Rahmstorf, and H. J. Schellnhuber, "Tipping elements in the earth's climate system", Proc. Nat. Acad. Sci. USA 105:6 (2008), 1786-1793.

[Marder and Goaillard 2006] E. Marder and J. M. Goaillard, "Variability, compensation and homeostasis in neuron and network function", Nat. Rev. Neurosci. 7 (2006), 563-574.

[Marković and Gros 2010] D. Marković and C. Gros, "Self-organized chaos through polyhomeostatic optimization”, Phys. Rev. Lett. 105:6 (2010), Article ID \#068702. arXiv 1001.0663

[Marković and Gros 2012] D. Marković and C. Gros, "Intrinsic adaptation in autonomous recurrent neural networks", Neural Comput. 24:2 (2012), 523-540.

[McDonnell and Abbott 2009] M. D. McDonnell and D. Abbott, "What is stochastic resonance? Definitions, misconceptions, debates, and its relevance to biology", PLoS Comput. Biol. 5:5 (2009), Article ID \#e1000348.

[Olshausen and Field 2004] B. A. Olshausen and D. J. Field, "Sparse coding of sensory inputs", Curr. Opin. Neurobiol. 14:4 (2004), 481-487.

[Plum et al. 2006] L. Plum, B. F. Belgardt, and J. C. BrÃ¹/4ning, "Central insulin action in energy and glucose homeostasis", J. Clin. Investig. 116:7 (2006), 1761-1766.

[Press et al. 2007] W. H. Press, S. A. Teukolsky, W. T. Vetterling, and B. P. Flannery, Numerical recipes: the art of scientific computing, 3rd ed., Cambridge University Press, Cambridge, 2007. 
[Schaefer 1961] E. K. Schaefer, "Blood $\mathrm{pH}$ and $\mathrm{pCO}_{2}$ homeostatis in chronic respiratory acidosis related to the use of amine and other buffers", Ann. NY Acad. Sci. 92 (1961), 401-413.

[Stemmler and Koch 1999] M. Stemmler and C. Koch, "How voltage-dependent conductances can adapt to maximize the information encoded by neuronal firing rate", Nat. Neurosci. 2:6 (1999), $521-527$.

[Tresguerres et al. 2010] M. Tresguerres, S. K. Parks, E. Salazar, L. R. Levin, G. G. Goss, and J. Buck, "Bicarbonate-sensing soluble adenylyl cyclase is an essential sensor for acid/base homeostasis”, Proc. Nat. Acad. Sci. USA 107:1 (2010), 442-447.

[Triesch 2005] J. Triesch, "A gradient rule for the plasticity of a neuron's intrinsic excitability", pp. 65-70 in Artificial neural networks: biological inspirations (ICANN 2005), I (Warsaw, 2005), edited by W. Duch et al., Lecture Notes in Computer Science 3696, Springer, Berlin, 2005.

Received 4 Apr 2012. Revised 13 Jul 2012. Accepted 3 Nov 2012.

MATHIAS LINKERHAND: linkerhand@th.physik.uni-frankfurt.de

Institute for Theoretical Physics, Goethe University, Max-von-Laue-Straße 1, D-60438 Frankfurt am Main, Germany

Claudius GRos: gros07@itp.uni-frankfurt.de

Institute for Theoretical Physics, Goethe University, Max-von-Laue-Straße 1, D-60438 Frankfurt am Main, Germany 


\title{
WELL-POSEDNESS FOR DISLOCATION-BASED GRADIENT VISCOPLASTICITY, II: GENERAL NONASSOCIATIVE MONOTONE PLASTIC FLOWS
}

\author{
SERGiY NESENENKO AND PATRIZIO NeFF
}

\begin{abstract}
In this work we extend the well-posedness for infinitesimal dislocation-based gradient viscoplasticity with linear kinematic hardening from the subdifferential case to general nonassociative monotone plastic flows. We assume an additive split of the displacement gradient into nonsymmetric elastic distortion and nonsymmetric plastic distortion. The thermodynamic potential is augmented with a term taking the dislocation density tensor Curl $p$ into account. The constitutive equations in the models we study are assumed to be only of monotone type. Based on the generalized version of Korn's inequality for incompatible tensor fields (the nonsymmetric plastic distortion) due to Neff et al. the existence of solutions of quasistatic initial-boundary value problems under consideration is shown using a time-discretization technique and a monotone operator method.
\end{abstract}

\section{Introduction}

We study the existence of solutions of quasistatic initial-boundary value problems arising in gradient viscoplasticity. The models we study use rate-dependent constitutive equations with internal variables to describe the deformation behavior of metals at infinitesimally small strain.

Our focus is on a phenomenological model on the macroscale not including the case of single-crystal plasticity. From a mathematical point of view, the maze of equations, slip systems, and physical mechanisms in single-crystal plasticity is only obscuring the mathematical structure of the problem.

Our model has been first presented in [Neff et al. 2009a]. It is inspired by [2000]. Contrary to more classical strain gradient approaches, the model features a nonsymmetric plastic distortion field $p \in M^{3}$ [Bardella 2010], a dislocation-based energy storage based solely on $|\mathrm{Curl} p|$, and second gradients of the plastic distortion in the

MSC2000: primary 35B65, 35D10, 74C10, 74D10; secondary 35J25, 34G20, 34G25, 47H04, $47 \mathrm{H} 05$.

Keywords: plasticity, gradient plasticity, viscoplasticity, rate-dependent response, nonassociative flow rule, dislocations, plastic spin, Rothe's time-discretization method, maximal monotone method, Korn's inequality for incompatible tensor fields. 
form of Curl Curl $p$ acting as dislocation-based kinematical backstresses. We only consider energetic length-scale effects and not higher gradients in the dissipation.

The uniqueness of classical solutions in the subdifferential case (associated plasticity) for rate-independent and rate-dependent formulations is shown in [Neff 2008b]. The existence question for the rate-independent model in terms of a weak reformulation is addressed in [Neff et al. 2009a]. The rate-independent model with isotropic hardening is treated in [Ebobisse and Neff 2010]. The first numerical results for a simplified rate-independent irrotational formulation (no plastic spin, symmetric plastic distortion $p$ ) are presented in [Neff et al. 2009b]. In [Giacomini and Lussardi 2008; Reddy et al. 2008] well-posedness for a rate-independent model of [Gurtin and Anand 2005] is shown under the decisive assumption that the plastic distortion is symmetric (the irrotational case), in which case we may really speak of a strain gradient plasticity model, since the gradient acts on the plastic strain.

In order to appreciate the simplicity and elegance of our model we sketch some of its ingredients. First, as is usual in plasticity theory, we split the total displacement gradient into nonsymmetric elastic and plastic distortions:

$$
\nabla u=e+p .
$$

For invariance reasons, the elastic energy contribution may only depend on the elastic strains $\operatorname{sym} e=\operatorname{sym}(\nabla u-p)$. While $p$ is nonsymmetric, a distinguishing feature of our model is that, similarly to classical approaches, only the symmetric part $\varepsilon_{p}:=\operatorname{sym} p$ of the plastic distortion appears in the local Cauchy stress $\sigma$, while the higher-order stresses are nonsymmetric. The reason for this is that we assume that $p$ has to obey the same transformation behavior as $\nabla u$ does, and thus the energy storage due to kinematical hardening should depend only on the plastic strains $\operatorname{sym} p$. For more on the basic invariance questions related to this issue dictating this type of behavior, see [Neff 2008a; Svendsen et al. 2009]. We assume as well plastic incompressibility: $\operatorname{tr} p=0$.

The thermodynamic potential of our model can therefore be written as

$$
\begin{aligned}
\int_{\Omega} \underbrace{(\underbrace{\mathbb{C}[x](\operatorname{sym}(\nabla u-p))(\operatorname{sym}(\nabla u-p))}_{\text {kinematical hardening }}}_{\text {elastic energy }} \\
+\underbrace{\frac{C_{1}}{2}|\operatorname{dev} \operatorname{sym} p|^{2}}_{\text {dislocation storage }}+\underbrace{\frac{C_{2}}{2}|\operatorname{Curl} p|^{2}}_{\text {external volume forces }}+\underbrace{u \cdot b}) d x .
\end{aligned}
$$

The positive definite elasticity tensor $\mathbb{C}$ is able to represent the elastic anisotropy of the material. The evolution equations for the plastic distortion $p$ are taken in such a way that the stored energy is nonincreasing along trajectories of $p$ at frozen displacement $u$; see [Neff et al. 2009a]. This ensures the validity of the second law 
of thermodynamics in the form of the reduced dissipation inequality.

For the reduced dissipation inequality we consider $u$ fixed in time and consider the time derivative of the free energy (and taking into account that Curl is a selfadjoint operator provided that the appropriate boundary conditions are specified), we have

$$
\begin{aligned}
\frac{d}{d t} \int_{\Omega} W\left(\nabla u\left(t_{0}\right)-p(t), p\right. & (t), \operatorname{Curl} p(t)) d x \\
= & \int_{\Omega} D_{1} W \cdot\left(-\partial_{t} p\right)+D_{2} W \cdot \partial_{t} p+D_{3} W \cdot \operatorname{Curl} \partial_{t} p d x \\
& =-\int_{\Omega}\left(D_{1} W-D_{2} W-\operatorname{Curl} D_{3} W\right) \cdot \partial_{t} p d x .
\end{aligned}
$$

Choosing $\partial_{t} p \in g\left(D_{1} W-D_{2} W-\operatorname{Curl} D_{3} W\right)$ with a monotone function $g$ we obtain the reduced dissipation inequality

$$
\frac{d}{d t} \int_{\Omega} W\left(\nabla u\left(t_{0}\right)-p(t), p(t), \operatorname{Curl} p(t)\right) d x \leq 0 .
$$

Adapted to our situation, the plastic flow has the form

$$
\partial_{t} p \in g\left(\sigma-C_{1} \operatorname{dev} \operatorname{sym} p-C_{2} \operatorname{Curl} \operatorname{Curl} p\right),
$$

where $\sigma=\mathbb{C}[x] \operatorname{sym}(\nabla u-p)$ is the elastic symmetric Cauchy stress of the material and $g$ is a multivalued monotone flow function which is not necessary the subdifferential of a convex plastic potential (associative plasticity). In this generality, our formulation comprises certain nonassociative plastic flows in which the yield condition and the flow direction are independent and governed by distinct functions. Moreover, the flow function $g$ is supposed to induce a rate-dependent response as all materials are rate dependent.

Clearly, in the absence of energetic length-scale effects $\left(C_{2}=0\right)$, the Curl Curl $p$ term is absent. In general we assume that $g$ maps symmetric tensors to symmetric tensors. Thus, for $C_{2}=0$ the plastic distortion remains symmetric and the model reduces to a classical plasticity model. Therefore, the energetic length scale is solely responsible for the plastic spin in the model. The appearance of the Curl Curl $p$ term in the argument of $g$ is clear: the argument of $g$ consists of the Eshelby stress tensor $\Sigma$ driving the plastic evolution, see [Neff et al. 2009a].

Regarding the boundary conditions necessary for the formulation of the higherorder theory we assume that the boundary is a perfect conductor, which means that the tangential component of $p$ vanishes on $\partial \Omega$. In the context of dislocation dynamics these conditions express the requirement that there is no flux of the Burgers vector across a hard boundary. Gurtin and Needleman [2005] introduce 
the following different types of boundary conditions for the plastic distortion: ${ }^{1}$

$$
\begin{aligned}
\partial_{t} p \times\left. n\right|_{\Gamma_{\text {hard }}}=0 & \text { "microhard" (perfect conductor), } \\
\left.\partial_{t} p\right|_{\Gamma_{\text {hard }}}=0 & \text { "hard-slip", } \\
\text { Curl } p \times\left. n\right|_{\Gamma_{\text {hard }}}=0 & \text { "microfree". }
\end{aligned}
$$

We specify a sufficient condition for the microhard boundary condition, namely

$$
p \times\left. n\right|_{\Gamma_{\text {hard }}}=0,
$$

and assume $\Gamma_{\text {hard }}=\partial \Omega$. This is the correct boundary condition for tensor fields in $H$ (Curl) which admits tangential traces.

We combine this with a new inequality extending Korn's inequality to incompatible tensor fields, namely, for all $p \in H$ (Curl) such that $p \times\left. n\right|_{\Gamma_{\text {hard }}}=0$, we have

$$
\underbrace{\|p\|_{L^{2}(\Omega)}}_{\text {plastic distortion }} \leq C(\Omega)(\underbrace{\|\operatorname{sym} p\|_{L^{2}(\Omega)}}_{\text {plastic strain }}+\underbrace{\|\operatorname{Curl} p\|_{L^{2}(\Omega)}}_{\text {dislocation density }}) .
$$

Here, $\Gamma_{\text {hard }} \subset \partial \Omega$ with full two-dimensional surface measure and domain $\Omega$ needs to be sliceable, that is, cuttable into finitely many simply connected subdomains with Lipschitz boundaries. This inequality has been derived in [Neff et al. 2011; $2012 \mathrm{a} ; 2012 \mathrm{~b}]$ and is precisely motivated by the well-posedness question for our model [Neff et al. 2009a]. Inequality (3) expresses the fact that controlling the plastic strain sym $p$ and the dislocation density $\operatorname{Curl} p$ in $L^{2}(\Omega)$ gives a control of the plastic distortion $p$ in $L^{2}(\Omega)$ provided the correct boundary conditions are specified: namely the microhard boundary condition. Since in the sequel we assume that $\operatorname{tr}(p)=0$ (plastic incompressibility) the quadratic terms in the thermodynamic potential provide a control of the right-hand side in (3).

It is worth noting that with $g$ only monotone and not necessarily a subdifferential the powerful energetic solution concept [Giacomini and Lussardi 2008; Mainik and Mielke 2009; Kratochvíl et al. 2010] cannot be applied. In this contribution we face the combined challenge of a gradient plasticity model based on the dislocation density tensor Curl $p$ involving the plastic spin, a general nonassociative monotone flow-rule, and a rate-dependent response.

Setting of the problem. Let $\Omega \subset \mathbb{R}^{3}$ be an open bounded set, the set of material points of the solid body, with a $C^{1}$-boundary. By $T_{e}$ we denote a positive number (time of existence), which can be chosen arbitrarily large, and for $0<t \leq T_{e}$,

$$
\Omega_{t}=\Omega \times(0, t) .
$$

\footnotetext{
${ }^{1}$ Here, $v \times n$ with $v \in M^{3}$ and where $n \in \mathbb{R}^{3}$ denotes a row by column operation.
} 
The sets $\mathcal{M}^{3}$ and $\mathscr{S}^{3}$ denote the sets of all $3 \times 3$ matrices and all symmetric $3 \times 3$ matrices, respectively. Let $\mathfrak{s l}(3)$ be the set of all traceless $3 \times 3$ matrices, that is,

$$
\mathfrak{s l}(3)=\left\{v \in M^{3} \mid \operatorname{tr} v=0\right\} .
$$

Unknown in our small strain formulation are the displacement $u(x, t) \in \mathbb{R}^{3}$ of the material point $x$ at time $t$ and the nonsymmetric infinitesimal plastic distortion $p(x, t) \in \mathfrak{s l}(3)$.

The model equations of the problem are

$$
\begin{aligned}
-\operatorname{div}_{x} \sigma(x, t) & =b(x, t), \\
\sigma(x, t) & =\mathbb{C}[x]\left(\operatorname{sym}\left(\nabla_{x} u(x, t)-p(x, t)\right)\right), \\
\partial_{t} p(x, t) & \in g\left(x, \Sigma^{\operatorname{lin}}(x, t)\right), \quad \Sigma^{\operatorname{lin}}=\Sigma_{e}^{\operatorname{lin}}+\Sigma_{\mathrm{sh}}^{\operatorname{lin}}+\Sigma_{\text {curl }}^{\operatorname{lin}}, \\
\Sigma_{\mathrm{e}}^{\operatorname{lin}} & =\sigma, \quad \Sigma_{\mathrm{sh}}^{\operatorname{lin}}=-C_{1} \operatorname{dev} \operatorname{sym} p, \quad \Sigma_{\mathrm{curl}}^{\operatorname{lin}}=-C_{2} \text { Curl Curl } p,
\end{aligned}
$$

which must be satisfied in $\Omega \times\left[0, T_{e}\right)$. Here, $C_{1}, C_{2} \geq 0$ are given material constants and $\Sigma^{\text {lin }}$ is the infinitesimal Eshelby stress tensor driving the evolution of the plastic distortion $p$. The initial condition and Dirichlet boundary condition are

$$
\begin{aligned}
p(x, 0) & =p^{(0)}(x), & x & \in \Omega, \\
p(x, t) \times n(x) & =0, & & (x, t) \in \partial \Omega \times\left[0, T_{e}\right), \\
u(x, t) & =0, & & (x, t) \in \partial \Omega \times\left[0, T_{e}\right),
\end{aligned}
$$

where $n$ is a normal vector on the boundary $\partial \Omega$. For simplicity we consider only the homogeneous boundary condition. The elasticity tensor $\mathbb{C}[x]: \mathscr{S}^{3} \rightarrow \mathscr{S}^{3}$ is a linear, symmetric, uniformly positive definite mapping. The mapping $x \mapsto \mathbb{C}[x]: \Omega \rightarrow \varphi^{3}$ is measurable. Classical linear kinematic hardening is included for $C_{1}>0$. Here, the nonlocal backstress contribution is given by the dislocation density motivated term $\Sigma_{\text {curl }}^{\operatorname{lin}}=-C_{2}$ Curl Curl $p$ together with the corresponding microhard boundary conditions.

For the model we require that the nonlinear constitutive mapping $(v \mapsto g(\cdot, v))$ : $\mathcal{M}^{3} \rightarrow 2^{\mathfrak{s l}(3)}$ is monotone ${ }^{2}$, that is, it satisfies

$$
0 \leq\left(v_{1}-v_{2}\right) \cdot\left(v_{1}^{*}-v_{2}^{*}\right),
$$

for all $v_{i} \in \mathcal{M}^{3}, v_{i}^{*} \in g\left(x, v_{i}\right), i=1,2$, and for a.e. $x \in \Omega$. We also require that

$$
0 \in g(x, 0), \quad \text { a.e. } x \in \Omega \text {. }
$$

The mapping $x \mapsto g(x, \cdot): \Omega \rightarrow 2^{\mathfrak{s l}(3)}$ is measurable (see Section 2 for the definition of the measurability of multivalued maps). Moreover, the function $g$ has the

\footnotetext{
${ }^{2}$ Here $2^{\mathfrak{s l}(3)}$ denotes the power set of $\mathfrak{s l}(3)$.
} 
following property:

$$
g(x, v) \in \mathscr{Y}^{3} \text { for any } v \in \mathscr{S}^{3} \text { and a.e. } x \in \Omega .
$$

Given are the volume force $b(x, t) \in \mathbb{R}^{3}$ and the initial datum $p^{(0)}(x) \in \mathfrak{s l}(3)$.

Remark 1.1. It is well known that classical viscoplasticity (without gradient effects) gives rise to a well-posed problem. We extend this result to our formulation of rate-dependent gradient plasticity. The presence of the classical linear kinematic hardening in our model is related to $C_{1}>0$ whereas the presence of the nonlocal gradient term is always related to $C_{2}>0$.

In the recent work by the authors [Nesenenko and Neff 2012] the existence of solutions for the initial boundary problem (4)-(9) is studied under the assumption that the monotone function $g$ is a subdifferential of a proper lower semicontinuous convex function $\phi: \mathcal{M}^{3} \rightarrow \overline{\mathbb{R}}(\overline{\mathbb{R}}:=\mathbb{R} \cup\{\infty\})$, that is, $g=\partial \phi$, and with the following different boundary condition:

$$
\operatorname{Curl} p(x, t) \times n(x)=0, \quad(x, t) \in \partial \Omega \times\left[0, T_{e}\right),
$$

instead of (8). It is required there that the function $\phi$ satisfies the following twosided estimate:

$$
a_{0}|v|^{q}-b_{0} \leq \phi(v) \leq a_{1}|v|^{q}+b_{1},
$$

for positive $a_{0}$ and $a_{1}$, some $b_{0}$ and $b_{1}$, and any $v \in \mathcal{M}^{3}$. Using methods of convex analysis we obtained in [Nesenenko and Neff 2012] the existence of weak solutions (see Definition 4.6) for the problem (4)-(7) + (12) + (9), with $g=\partial \phi$, under the restrictions on $g$ given above. We note that the existence result derived in that paper is also valid for the new problem (4)-(9), that is, with the boundary condition (8) instead of (12), and of course the subdifferential structural assumption on $g$. In this work, assuming $\Omega \subset \mathbb{R}^{3}$ is a sliceable domain with a $C^{1}$-boundary and the homogeneous initial condition for $p$, that is, $p^{(0)}(x)=0$ for $x \in \Omega$, we show the existence of strong solutions (see Definition 4.5) for the problem (4)-(9) with the monotone function $g$ belonging to the class $\mathbb{M}\left(\Omega, M^{3}, q, \alpha, m\right)$ defined in Section 4. The derivation of this result is based on the inequality (3), which is recently obtained in [Neff et al. 2011;2012c] under the assumption that $\Omega$ is a sliceable domain, and on the monotonicity assumption for the function $g$. We note that in the case of the sliceable domain $\Omega$ the methods used in this work allow us to show the existence of strong solutions for (4)-(9) with $g=\partial \phi$, that is, the weak solutions for (4)-(9) with $g=\partial \phi$ derived in [Nesenenko and Neff 2012] are the strong solutions in the sense of Definition 4.5 in this case. However, we do not know how to extend our results on the existence of strong solutions to domains $\Omega$ which are not sliceable and to the nonhomogeneous initial condition. We note as well that the existence of strong solutions for the initial boundary problem formed 
by (4)-(7), (9), and (12), with $g \in \mathbb{M}\left(\Omega, M^{3}, q, \alpha, m\right)$ or $g=\partial \phi$ with $\phi$ satisfying (13) for any domain $\Omega$, is an open problem too.

Notation. Throughout we choose the numbers $q$ and $q^{*}$ satisfying the following conditions:

$$
1<q, q^{*}<\infty \quad \text { and } \quad 1 / q+1 / q^{*}=1,
$$

where $|\cdot|$ denotes a norm in $\mathbb{R}^{k}, k \in \mathbb{N}$. Moreover, the following notations are used in this work. The space $W^{m, q}\left(\Omega, \mathbb{R}^{k}\right)$ with $q \in[1, \infty]$ consists of all functions in $L^{q}\left(\Omega, \mathbb{R}^{k}\right)$ with weak derivatives in $L^{q}\left(\Omega, \mathbb{R}^{k}\right)$ up to order $m$. If $m$ is not integer, then $W^{m, q}\left(\Omega, \mathbb{R}^{k}\right)$ denotes the corresponding Sobolev-Slobodecki space. We set $H^{m}\left(\Omega, \mathbb{R}^{k}\right)=W^{m, 2}\left(\Omega, \mathbb{R}^{k}\right)$. The norm in $W^{m, q}\left(\Omega, \mathbb{R}^{k}\right)$ is denoted by $\|\cdot\|_{m, q, \Omega}$ $\left(\|\cdot\|_{q}:=\|\cdot\|_{0, q, \Omega}\right)$. The operator $\Gamma_{0}$ defined by

$$
\Gamma_{0}: v \in W^{1, q}\left(\Omega, \mathbb{R}^{k}\right) \mapsto W^{1-1 / q, q}\left(\partial \Omega, \mathbb{R}^{k}\right)
$$

denotes the usual trace operator. The space $W_{0}^{m, q}\left(\Omega, \mathbb{R}^{k}\right)$ with $q \in[1, \infty]$ consists of all functions $v$ in $W^{m, q}\left(\Omega, \mathbb{R}^{k}\right)$ with $\Gamma_{0} v=0$. One can define the bilinear form on the product space $L^{q}\left(\Omega, M^{3}\right) \times L^{q^{*}}\left(\Omega, M^{3}\right)$ by

$$
(\xi, \zeta)_{\Omega}=\int_{\Omega} \xi(x) \cdot \zeta(x) d x .
$$

The space

$$
L_{\text {Curl }}^{q}\left(\Omega, M^{3}\right)=\left\{v \in L^{q}\left(\Omega, M^{3}\right) \mid \operatorname{Curl} v \in L^{q}\left(\Omega, M^{3}\right)\right\}
$$

is a Banach space with respect to the norm

$$
\|v\|_{q, \mathrm{Curl}}=\|v\|_{q}+\|\operatorname{Curl} v\|_{q} .
$$

By $H(\mathrm{Curl})$ we denote the space of measurable functions in $L_{\text {Curl }}^{2}\left(\Omega, M^{3}\right)$, that is, $H(\mathrm{Curl})=L_{\text {Curl }}^{2}\left(\Omega, M^{3}\right)$. The well-known result on the generalized trace operator can be easily adapted to functions with values in $\mathcal{M}^{3}$ (see [Sohr 2001, §II.1.2]). Then, according to this result, there is a bounded operator $\Gamma_{n}$ on $L_{\text {Curl }}^{q}\left(\Omega, M^{3}\right)$ :

$$
\Gamma_{n}: v \in L_{\text {Curl }}^{q}\left(\Omega, M^{3}\right) \mapsto\left(W^{1-1 / q^{*}, q^{*}}\left(\partial \Omega, M^{3}\right)\right)^{*}
$$

with

$$
\Gamma_{n} v=v \times\left. n\right|_{\partial \Omega} \text { if } v \in C^{1}\left(\bar{\Omega}, M^{3}\right),
$$

where $X^{*}$ denotes the dual of a Banach space $X$. Next,

$$
L_{\text {Curl }, 0}^{q}\left(\Omega, M^{3}\right)=\left\{w \in L_{\text {Curl }}^{q}\left(\Omega, M^{3}\right) \mid \Gamma_{n}(w)=0\right\} .
$$

We also define the space $Z_{\text {Curl }}^{q}\left(\Omega, M^{3}\right)$ by

$$
Z_{\text {Curl }}^{q}\left(\Omega, M^{3}\right)=\left\{v \in L_{\text {Curl }, 0}^{q}\left(\Omega, M^{3}\right) \mid \operatorname{Curl} \operatorname{Curl} v \in L^{q}\left(\Omega, M^{3}\right)\right\},
$$


which is a Banach space with respect to the norm

$$
\|v\|_{Z_{\text {Curl }}^{q}}=\|v\|_{q, \text { Curl }}+\|\operatorname{Curl} \operatorname{Curl} v\|_{q} .
$$

For functions $v$ defined on $\Omega \times[0, \infty)$ we denote by $v(t)$ the mapping $x \mapsto v(x, t)$, which is defined on $\Omega$. The space $L^{q}\left(0, T_{e} ; X\right)$ denotes the Banach space of all Bochner-measurable functions $u:\left[0, T_{e}\right) \rightarrow X$ such that $t \mapsto\|u(t)\|_{X}^{q}$ is integrable on $\left[0, T_{e}\right)$. Finally, we frequently use the spaces $W^{m, q}\left(0, T_{e} ; X\right)$, which consist of Bochner-measurable functions having $q$-integrable weak derivatives up to order $m$.

\section{Maximal monotone operators}

In this section we recall some basics about monotone and maximal monotone operators. For more details see [Barbu 1976; Pascali and Sburlan 1978; Hu and Papageorgiou 1997], for example.

Let $V$ be a reflexive Banach space with the norm $\|\cdot\|$, and let $V^{*}$ be its dual space with the norm $\|\cdot\|_{*}$. The brackets $\langle\cdot, \cdot\rangle$ denote the dual pairing between $V$ and $V^{*}$. Under $V$ we shall always mean a reflexive Banach space throughout this section. For a multivalued mapping $A: V \rightarrow 2^{V^{*}}$ we define the effective domain of $A$ as

$$
D(A)=\{v \in V \mid A v \neq \varnothing\}
$$

and the graph of $A$ as

$$
\text { Gr } A=\left\{\left[v, v^{*}\right] \in V \times V^{*} \mid v \in D(A), v^{*} \in A v\right\} .
$$

Definition 2.1. A mapping $A: V \rightarrow 2^{V^{*}}$ is called monotone if the inequality

$$
\left\langle v^{*}-u^{*}, v-u\right\rangle \geq 0
$$

holds for all $\left[v, v^{*}\right],\left[u, u^{*}\right] \in \operatorname{Gr} A$. A monotone mapping $A: V \rightarrow 2^{V^{*}}$ is called maximal monotone if the inequality

$$
\left\langle v^{*}-u^{*}, v-u\right\rangle \geq 0 \text { for all }\left[u, u^{*}\right] \in \operatorname{Gr} A
$$

implies $\left[v, v^{*}\right] \in \mathrm{Gr} A$.

A mapping $A: V \rightarrow 2^{V^{*}}$ is called generalized pseudomonotone if the set $A v$ is closed, convex, and bounded for all $v \in D(A)$ and, for every pair of sequences $\left\{v_{n}\right\}$ and $\left\{v_{n}^{*}\right\}$ such that $v_{n}^{*} \in A v_{n}, v_{n} \rightarrow v_{0}, v_{n}^{*} \rightarrow v_{0}^{*} \in V^{*}$ and

$$
\limsup _{n \rightarrow \infty}\left\langle v_{n}^{*}, v_{n}-v_{0}\right\rangle \leq 0
$$

we have $\left[v_{0}, v_{0}^{*}\right] \in \operatorname{Gr} A$ and $\left\langle v_{n}^{*}, v_{n}\right\rangle \rightarrow\left\langle v_{0}^{*}, v_{0}\right\rangle$. 
A mapping $A: V \rightarrow 2^{V^{*}}$ is called strongly coercive if either $D(A)$ is bounded or $D(A)$ is unbounded and the condition

$$
\frac{\left\langle v^{*}, v-w\right\rangle}{\|v\|} \rightarrow+\infty \quad \text { as } \quad\|v\| \rightarrow \infty, \quad\left[v, v^{*}\right] \in \operatorname{Gr} A,
$$

is satisfied for each $w \in D(A)$.

It is well known [Pascali and Sburlan 1978, p. 105] that if $A$ is a maximal monotone operator, then for any $v \in D(A)$ the image $A v$ is a closed convex subset of $V^{*}$ and the graph $\mathrm{Gr} A$ is demiclosed. ${ }^{3}$ A maximal monotone operator is also generalized pseudomonotone; see [Barbu 1976; Pascali and Sburlan 1978; Hu and Papageorgiou 1997].

Remark 2.2. We recall that the subdifferential of a lower semicontinuous and convex function is maximal monotone; see, for example, [Phelps 1993, Theorem 2.25].

Definition 2.3. The duality mapping $J: V \rightarrow 2^{V^{*}}$ is defined by

$$
J(v)=\left\{v^{*} \in V^{*} \mid\left\langle v^{*}, v\right\rangle=\|v\|^{2}=\left\|v^{*}\right\|_{*}^{2}\right\}
$$

for all $v \in V$.

Without loss of generality (due to Asplund's theorem) we can assume that both $V$ and $V^{*}$ are strictly convex, that is, that the unit ball in the corresponding space is strictly convex. By virtue of [Barbu 1976, Theorem II.1.2], the equation

$$
J\left(v_{\lambda}-v\right)+\lambda A v_{\lambda} \ni 0
$$

has a solution $v_{\lambda} \in D(A)$ for every $v \in V$ and $\lambda>0$ if $A$ is maximal monotone. The solution is unique; see [Barbu 1976, p. 41].

Definition 2.4. Setting

$$
v_{\lambda}=j_{\lambda}^{A} v \quad \text { and } \quad A_{\lambda} v=-\lambda^{-1} J\left(v_{\lambda}-v\right)
$$

we define two single-valued operators: the Yosida approximation $A_{\lambda}: V \rightarrow V^{*}$ and the resolvent $j_{\lambda}^{A}: V \rightarrow D(A)$ with $D\left(A_{\lambda}\right)=D\left(j_{\lambda}^{A}\right)=V$.

By this definition, one immediately sees that $A_{\lambda} v \in A\left(j_{\lambda}^{A} v\right)$. For the main properties of the Yosida approximation we refer to [Barbu 1976; Pascali and Sburlan 1978; $\mathrm{Hu}$ and Papageorgiou 1997] and mention only that both are continuous operators and that $A_{\lambda}$ is bounded and maximal monotone.

Next, the maximality of the sum of two maximal monotone operators is given by the following result.

${ }^{3}$ A set $A \in V \times V^{*}$ is demiclosed if, whenever $v_{n}$ converges strongly to $v_{0}$ in $V$ and $v_{n}^{*}$ converges weakly to $v_{0}^{*}$ in $V^{*}$ (or $v_{n}$ converges weakly to $v_{0}$ in $V$ and $v_{n}^{*}$ converges strongly to $v_{0}^{*}$ in $V^{*}$ ) and $\left[v_{n}, v_{n}^{*}\right] \in \operatorname{Gr} A$, we have $\left[v, v^{*}\right] \in \operatorname{Gr} A$. 
Theorem 2.5. Let $V$ be a reflexive Banach space, and let $A$ and $B$ be maximal. Suppose that the condition

$$
D(A) \cap \operatorname{int} D(B) \neq \varnothing
$$

is fulfilled. Then the sum $A+B$ is a maximal monotone operator.

Proof. See [Pascali and Sburlan 1978, Theorem III.3.6] or [Barbu 1976, Theorem II.1.7].

For deeper results on the maximality of the sum of two maximal monotone operators we refer the reader to the book [Simons 1998]. The next surjectivity result plays an important role in the existence theory for monotone operators.

Theorem 2.6. If $V$ is a (strictly convex) reflexive Banach space and $A: V \rightarrow 2^{V^{*}}$ is maximal monotone and coercive, then $A$ is surjective.

Proof. See [Pascali and Sburlan 1978, Theorem III.2.10].

Measurability of multivalued mappings. In this subsection we present briefly some facts about measurable multivalued mappings. We assume that $V$, and hence $V^{*}$, is separable and denote the set of maximal monotone operators from $V$ to $V^{*}$ by $\mathfrak{M}\left(V \times V^{*}\right)$. Further, let $(S, \Sigma(S), \mu)$ be a $\sigma$-finite $\mu$-complete measurable space.

Definition 2.7. A function $A: S \rightarrow \mathfrak{M}\left(V \times V^{*}\right)$ is measurable if, for every open set $U \in V \times V^{*}$, the set

$$
\{x \in S \mid A(x) \cap U \neq \varnothing\}
$$

is measurable in $S$. Here "open set" could be replaced by "closed set", "Borel set", "open ball", or "closed ball", with an equivalent result.

The next result states that the notion of measurability for maximal monotone mappings can be equivalently defined in terms of the measurability for appropriate single-valued mappings.

Proposition 2.8. Let $A: S \rightarrow \mathfrak{M}\left(V \times V^{*}\right)$, let $\lambda>0$ and let $E$ be dense in $V$. The following are equivalent:

(a) A is measurable,

(b) for every $v \in E, x \mapsto j_{\lambda}^{A(x)} v$ is measurable, and

(c) $v \in E, x \mapsto A_{\lambda}(x) v$ is measurable.

Proof. See [Damlamian et al. 2007, Proposition 2.11].

For further reading on measurable multivalued mappings we refer the reader to [Castaing and Valadier 1977; Hu and Papageorgiou 1997; Pankov 1997]. 
Canonical extensions of maximal monotone operators. Given a mapping

$$
A: S \rightarrow \mathfrak{M}\left(V \times V^{*}\right),
$$

one can define a monotone graph from $L^{q}(S, V)$ to $L^{q^{*}}\left(S, V^{*}\right)$, where $1 / q+$ $1 / q^{*}=1$, as follows.

Definition 2.9. Let $A: S \rightarrow \mathfrak{M}\left(V \times V^{*}\right)$, the canonical extension of $A$ from $L^{q}(S, V)$ to $L^{q^{*}}\left(S, V^{*}\right)$, where $1 / q+1 / q^{*}=1$, is defined by:

$\operatorname{Gr} \mathscr{A}=\left\{\left[v, v^{*}\right] \in L^{p}(S, V) \times L^{q}\left(S, V^{*}\right) \mid\left[v(x), v^{*}(x)\right] \in \operatorname{Gr} A(x)\right.$ for a.e. $\left.x \in S\right\}$.

Monotonicity of $\mathscr{A}$ as defined in Definition 2.9 is obvious, while its maximality follows from the next proposition.

Proposition 2.10. Let $A: S \rightarrow \mathfrak{M}\left(V \times V^{*}\right)$ be measurable. If $\operatorname{Gr} \mathscr{A} \neq \varnothing$, then $\mathscr{A}$ is maximal monotone.

Proof. See [Damlamian et al. 2007, Proposition 2.13].

We have to point out here that the maximality of $A(x)$ for almost every $x \in S$ does not imply the maximality of $\mathscr{A}$ as the latter can be empty [Damlamian et al. 2007]: $S=(0,1)$ and $\operatorname{Gr} A(x)=\left\{\left[v, v^{*}\right] \in \mathbb{R}^{m} \times \mathbb{R}^{m} \mid v^{*}=x^{-1 / q}\right\}$.

\section{Some properties of the Curl Curl operator}

In this section we present some results concerning the Curl Curl operator, which are relevant to further investigations. For the Curl Curl operator with a slightly different domain of definition similar results are obtained in [Nesenenko and Neff $2012, \S 4]$. Here we adopt the results of that papers to our purposes.

Lemma 3.1 (self-adjointness of Curl Curl). Let $\Omega \subset \mathbb{R}^{3}$ be an open bounded set with a Lipschitz boundary and $A: L^{2}\left(\Omega, M^{3}\right) \rightarrow L^{2}\left(\Omega, M^{3}\right)$ be the linear operator defined by

$$
A v=\operatorname{Curl} \operatorname{Curl} v
$$

with $\operatorname{dom}(A)=Z_{\text {Curl }}^{2}\left(\Omega, M^{3}\right)$. The operator $A$ is self-adjoint and nonnegative.

Proof. Consider the closed linear operator $S: L^{2}\left(\Omega, M^{3}\right) \rightarrow L^{2}\left(\Omega, M^{3}\right)$ defined by

$$
S v=\operatorname{Curl} v, \quad v \in \operatorname{dom}(S)=L_{\operatorname{Curl}, 0}^{2}\left(\Omega, M^{3}\right) .
$$

It is easily seen that its adjoint is given by

$$
S^{*} v=\operatorname{Curl} v, \quad v \in \operatorname{dom}\left(S^{*}\right)=L_{\text {Curl }}^{2}\left(\Omega, M^{3}\right) .
$$

Then, by [Kato 1966, Theorem V.3.24], the operator $S^{*} S$ with

$$
\operatorname{dom}\left(S^{*} S\right)=\left\{v \in \operatorname{dom}(S) \mid S v \in \operatorname{dom}\left(S^{*}\right)\right\},
$$


which is exactly the operator $A$, is self-adjoint in $L^{2}\left(\Omega, M^{3}\right)$. The nonnegativity of $A$ follows from its representation by the operator $S$, that is, $A=S^{*} S$, and the identity

$$
(A v, u)_{\Omega}=\left(S^{*} S v, u\right)_{\Omega}=(S v, S u)_{\Omega},
$$

which holds for all $v \in \operatorname{dom}(A)$ and $u \in \operatorname{dom}(S)$.

Corollary 3.2. The operator $A: L^{2}\left(\Omega, M^{3}\right) \rightarrow L^{2}\left(\Omega, M^{3}\right)$ defined in Lemma 3.1 is maximal monotone.

Proof. According to [Brézis 1970, Theorem 1], a linear monotone operator $A$ is maximal monotone if it is a densely defined closed operator whose adjoint $A^{*}$ is monotone. The statement of the corollary follows then directly from Lemma 3.1 and the mentioned result of Brézis.

Boundary value problems. Let $\Omega \subset \mathbb{R}^{3}$ be an open bounded set with a Lipschitz boundary. For every $v \in L^{2}\left(\Omega, M^{3}\right)$ we define a functional $\Psi$ on $L^{2}\left(\Omega, M^{3}\right)$ by

$$
\Psi(v)= \begin{cases}\frac{1}{2} \int_{\Omega}|\operatorname{Curl} v(x)|^{2} d x, & v \in L_{\text {Curl }, 0}^{2}\left(\Omega, M^{3}\right), \\ +\infty, & \text { otherwise. }\end{cases}
$$

It is easy to check that $\Psi$ is proper, convex, and lower semicontinuous. The next lemma gives a precise description of the subdifferential $\partial \Psi$.

Lemma 3.3. We have that $\partial \Psi=$ Curl Curl with

$$
\operatorname{dom}(\partial \Psi)=Z_{\text {Curl }}^{2}\left(\Omega, M^{3}\right) .
$$

Proof. Let $A: L^{2}\left(\Omega, M^{3}\right) \rightarrow L^{2}\left(\Omega, M^{3}\right)$ be the linear operator defined by

$$
A v=\operatorname{Curl} \operatorname{Curl} v
$$

and $\operatorname{dom}(A)=Z_{\mathrm{Curl}}^{2}\left(\Omega, \mathcal{M}^{3}\right)$. Due to Lemma 3.1, the identity

$$
\int_{\Omega} \operatorname{Curl} \operatorname{Curl} v(x) \cdot w(x) d x=\int_{\Omega} \operatorname{Curl} v(x) \cdot \operatorname{Curl} w(x) d x
$$

holds for any $v, w \in Z_{\text {Curl }}^{2}\left(\Omega, M^{3}\right)$. Therefore, using (14) we obtain

$$
\int_{\Omega} \operatorname{Curl} \operatorname{Curl} v \cdot(w-v) d x=\int_{\Omega} \operatorname{Curl} v \cdot(\operatorname{Curl} w-\operatorname{Curl} v) d x \leq \Psi(w)-\Psi(v)
$$

for every $v, w \in \operatorname{dom}(A)$. This shows that $A \subset \partial \Psi$. Since $A$ is maximal monotone (see Corollary 3.2) we conclude that $A=\partial \Psi$. 


\section{Existence of strong solutions}

In this section we prove the main existence result for (4)-(9). To show the existence of weak solutions a time-discretization method is used in this work. In the first step, we prove the existence of the solutions of the time-discretized problem in appropriate Hilbert spaces based on the Helmholtz projection in $L^{2}\left(\Omega, \varphi^{3}\right)$ (Appendix A) and monotone operator methods (Section 2). In order to be able to apply the monotone operator method to the time-discretized problem we regularize it by a linear positive definite term. In the second step, we derive the uniform a priori estimates for the solutions of the time-discretized problem using the polynomial growth of the function $g$ (see Definition 4.1) and then we pass to the weak limit in the equivalent formulation of the time-discretized problem employing the weak lower semicontinuity of lower semicontinuous convex functions and the maximal monotonicity of $g$.

Main result. First, we define the class of maximal monotone functions we deal with in this work.

Definition 4.1. For $m \in L^{1}(\Omega, \mathbb{R}), \alpha \in \mathbb{R}_{+}$, and $q>1, \mathbb{M}\left(\Omega, \mathbb{R}^{k}, q, \alpha, m\right)$ is the set of multivalued functions $h: \Omega \times \mathbb{R}^{k} \rightarrow 2^{\mathbb{R}^{k}}$ with the following properties:

- $v \mapsto h(x, v)$ is maximal monotone for almost all $x \in \Omega$,

- the mapping $x \mapsto j_{\lambda}(x, v): \Omega \rightarrow \mathbb{R}^{k}$ is measurable for all $\lambda>0$, where $j_{\lambda}(x, v)$ is the inverse of $v \mapsto v+\lambda h(x, v)$,

- for a.e. $x \in \Omega$ and every $v^{*} \in h(x, v)$

$$
\alpha\left(\frac{|v|^{q}}{q}+\frac{\left|v^{*}\right|^{q^{*}}}{q^{*}}\right) \leq\left(v, v^{*}\right)+m(x)
$$

where $1 / q+1 / q^{*}=1$.

Remark 4.2. The condition (15) is equivalent to the following two inequalities:

$$
\left|v^{*}\right|^{q^{*}} \leq m_{1}(x)+\alpha_{1}|v|^{q}, \quad\left(v, v^{*}\right) \geq m_{2}(x)+\alpha_{2}|v|^{q},
$$

for a.e. $x \in \Omega$ and every $v^{*} \in h(x, v)$ and with suitable functions $m_{1}, m_{2} \in L^{1}(\Omega, \mathbb{R})$ and numbers $\alpha_{1}, \alpha_{2} \in \mathbb{R}_{+}$.

Remark 4.3. Viscoplasticity is typically included in the former conditions by choosing the function $g$ to be in Norton-Hoff form, that is,

$$
g(\Sigma)=\left[|\Sigma|-\sigma_{\mathrm{y}}\right]_{+}^{r} \frac{\Sigma}{|\Sigma|}, \quad \Sigma \in \mathcal{M}^{3},
$$

where $\sigma_{\mathrm{y}}$ is the flow stress and $r$ is some parameter together with $[x]_{+}:=\max (x, 0)$. If $g: M^{3} \mapsto \mathscr{9}^{3}$ then the flow is called irrotational (no plastic spin). 
In case of a nonassociative flow rule, $g$ is not a subdifferential but may, for example, be written as

$$
g(\Sigma)=\mathscr{F}_{1}(\Sigma) \partial \mathscr{F}_{2}(\Sigma),
$$

where $\mathscr{F}_{1}$ describes the yield function and $\mathscr{F}_{2}$ the flow direction.

The main properties of the class $\mathbb{M}\left(\Omega, \mathbb{R}^{k}, q, \alpha, m\right)$ are collected in the following proposition.

Proposition 4.4. Let $\mathscr{H}$ be a canonical extension of a function $h: \mathbb{R}^{k} \rightarrow 2^{\mathbb{R}^{k}}$, which belongs to $\mathbb{M}\left(\Omega, \mathbb{R}^{k}, q, \alpha, m\right)$. Then $\mathcal{H}$ is maximal monotone and surjective, and $D(\mathscr{H})=L^{p}\left(\Omega, \mathbb{R}^{k}\right)$.

Proof. See [Damlamian et al. 2007, Corollary 2.15].

Next, we define two notions of solution for the initial boundary value problem (4)-(9). Both notions are introduced without assuming the homogeneity of the initial condition (7).

Definition 4.5 (strong solution). A function $(u, \sigma, p)$ such that

$$
\begin{gathered}
(u, \sigma) \in H^{1}\left(0, T_{e} ; H_{0}^{1}\left(\Omega, \mathbb{R}^{3}\right) \times L^{2}\left(\Omega, \varphi^{3}\right)\right), \quad \Sigma^{\operatorname{lin}} \in L^{q}\left(\Omega_{T_{e}}, M^{3}\right), \\
p \in H^{1}\left(0, T_{e} ; L_{\text {Curl }}^{2}\left(\Omega, M^{3}\right)\right) \cap L^{2}\left(0, T_{e} ; Z_{\text {Curl }}^{2}\left(\Omega, M^{3}\right)\right),
\end{gathered}
$$

is called a strong solution of the initial boundary value problem (4)-(9) if, for every $t \in\left[0, T_{e}\right]$, the function $(u(t), \sigma(t))$ is a weak solution of the boundary value problem (73) with $\hat{\varepsilon}_{p}=\operatorname{sym} p(t)$, and the condition $\hat{b}=b(t)$, the evolution inclusion (6) and the initial condition (7) are satisfied pointwise.

For the reader's convenience we give here also the definition of a weak solution for the problem (4)-(9) in the case when the monotone function $g$ is a subdifferential of a proper lower semicontinuous convex function $\phi$, that is, $g=\partial \phi$.

Definition 4.6 (weak solution). A function $(u, \sigma, p)$ such that

$$
\begin{gathered}
(u, \sigma) \in W^{1, q^{*}}\left(0, T_{e} ; W_{0}^{1, q^{*}}\left(\Omega, \mathbb{R}^{3}\right) \times L^{q^{*}}\left(\Omega, \mathscr{S}^{3}\right)\right), \quad \Sigma^{\operatorname{lin}} \in L^{q}\left(\Omega_{T_{e}}, M^{3}\right), \\
p \in W^{1, q^{*}}\left(0, T_{e} ; L^{q^{*}}\left(\Omega, \mathcal{M}^{3}\right)\right) \cap L^{q^{*}}\left(0, T_{e} ; Z_{\text {Curl }}^{q^{*}}\left(\Omega, M^{3}\right)\right),
\end{gathered}
$$

with

$$
(\sigma, \operatorname{dev} \operatorname{sym} p, \operatorname{Curl} p) \in L^{\infty}\left(0, T_{e} ; L^{2}\left(\Omega, \mathscr{S}^{3} \times \mathcal{M}^{3} \times \mathcal{M}^{3}\right)\right),
$$

is called a weak solution of the initial boundary value problem (4)-(9) if for every $t \in\left[0, T_{e}\right]$ the function $(u(t), \sigma(t))$ is a weak solution of the boundary value problem (73) with $\hat{\varepsilon}_{p}=\operatorname{sym} p(t)$ and $\hat{b}=b(t)$, the initial condition (7) is satisfied, and 
the inequality 4

$$
\begin{aligned}
& \frac{1}{2} \int_{\Omega} \mathbb{C}^{-1}[x] \sigma(x, t) \cdot \sigma(x, t) d x+C_{1}\|\operatorname{dev} \operatorname{sym} p(t)\|_{2}^{2}+C_{2}\|\operatorname{Curl} p(t)\|_{2}^{2} \\
& +\int_{0}^{t} \int_{\Omega}\left(\phi^{*}\left(x, \partial_{s} p(x, s)\right)+\phi\left(x, \Sigma^{\operatorname{lin}}(x, s)\right)\right) d x d s \leq \int_{0}^{t}\left(b(s), \partial_{s} u(s)\right)_{\Omega} d s \\
& \quad+\frac{1}{2} \int_{\Omega} \mathbb{C}^{-1}[x] \sigma^{(0)}(x) \cdot \sigma^{(0)}(x) d x+C_{1}\left\|\operatorname{dev} \operatorname{sym} p^{(0)}\right\|_{2}^{2}+C_{2}\left\|\operatorname{Curl} p^{(0)}\right\|_{2}^{2}
\end{aligned}
$$

holds for all $t \in\left(0, T_{e}\right)$, with the function $\sigma^{(0)} \in L^{2}\left(\Omega, \varphi^{3}\right)$ determined by (73) for $\hat{\varepsilon}_{p}=\operatorname{sym} p^{(0)}$ and $\hat{b}=b(0)$.

In our previous paper [Nesenenko and Neff 2012] it is shown that under some additional regularity the weak solutions of the problem (4)-(9) with $g=\partial \phi$ become strong solutions of (4)-(9) in the sense of Definition 4.5.

Next, we state the main result of this work.

Theorem 4.7. Suppose that $1<q^{*} \leq 2 \leq q<\infty$. Assume that $\Omega \subset \mathbb{R}^{3}$ is a sliceable domain with a $C^{1}$-boundary and $\mathbb{C} \in L^{\infty}\left(\Omega, \varphi^{3}\right)$. Let the functions $b \in$ $W^{1, q}\left(0, T_{e} ; L^{q}\left(\Omega, \mathbb{R}^{3}\right)\right)$ be given. Assume that $g \in \mathbb{M}\left(\Omega, M^{3}, q, \alpha, m\right)$ and that for a.e. $x \in \Omega$ the relations

$$
p^{(0)}(x)=0 \quad \text { and } \quad 0 \in g\left(x, \sigma^{(0)}(x)\right)
$$

hold, where the function $\sigma^{(0)} \in L^{2}\left(\Omega, \varphi^{3}\right)$ is determined by (73) for $\hat{\varepsilon}_{p}=0$ and $\hat{b}=b(0)$.

Then there exists a solution $(u, \sigma, p)$ of the initial boundary value problem (4)-(9).

In order to deal with the measurable elasticity tensor $\mathbb{C}$, we reformulate the problem (4)-(9) as follows: Let the function $(\hat{v}, \hat{\sigma}) \in W^{1, q}\left(0, T_{e}, W_{0}^{1, q}\left(\Omega, \mathbb{R}^{3}\right) \times\right.$ $\left.L^{q}\left(\Omega, \mathscr{S}^{3}\right)\right)$ be a solution of the linear elasticity problem formed by

$$
\begin{aligned}
-\operatorname{div}_{x} \hat{\sigma}(x, t) & =b(x, t), & & x \in \Omega, \\
\hat{\sigma}(x, t) & =\hat{\mathbb{C}}\left(\operatorname{sym}\left(\nabla_{x} \hat{v}(x, t)\right),\right. & & x \in \Omega, \\
\hat{v}(x, t) & =0, & & x \in \partial \Omega,
\end{aligned}
$$

where $\hat{\mathbb{C}}: \mathscr{S}^{3} \rightarrow \mathscr{S}^{3}$ is any positive definite linear mapping independent of $(x, t)$. Such a function $(\hat{v}, \hat{\sigma})$ exists (see Appendix A). Then the solution $(u, \sigma, p)$ of the initial boundary value problem (4)-(9) has the form

$$
(u, \sigma, p)=(\tilde{v}+\hat{v}, \tilde{\sigma}+\hat{\sigma}, p),
$$

where the function $(\tilde{v}, \tilde{\sigma}, p)$ solves the problem

\footnotetext{
${ }^{4}$ Here $\phi^{*}$ is the Legendre-Fenchel conjugate of $\phi$.
} 


$$
\begin{aligned}
& -\operatorname{div}_{x} \tilde{\sigma}(x, t)=0, \\
& \tilde{\sigma}(x, t)=\mathbb{C}[x]\left(\operatorname{sym}\left(\nabla_{x} \tilde{v}(x, t)-p(x, t)\right)\right)+(\mathbb{C}[x]-\hat{\mathbb{C}})\left(\operatorname{sym}\left(\nabla_{x} \hat{v}(x, t)\right)\right), \\
& \partial_{t} p(x, t) \in g\left(x, \Sigma^{\operatorname{lin}}(x, t)\right), \quad \Sigma^{\operatorname{lin}}=\Sigma_{e}^{\operatorname{lin}}+\Sigma_{\mathrm{sh}}^{\operatorname{lin}}+\Sigma_{\text {curl }}^{\operatorname{lin}} \\
& \left(\Sigma_{\mathrm{e}}^{\operatorname{lin}}=\tilde{\sigma}+\hat{\sigma}, \quad \Sigma_{\mathrm{sh}}^{\operatorname{lin}}=-C_{1} \operatorname{dev} \operatorname{sym} p, \quad \Sigma_{\text {curl }}^{\operatorname{lin}}=-C_{2} \text { Curl Curl } p\right), \\
& p(x, 0)=0, \quad x \in \Omega, \\
& p(x, t) \times n(x)=0, \quad(x, t) \in \partial \Omega \times\left[0, T_{e}\right), \\
& \tilde{v}(x, t)=0, \quad(x, t) \in \partial \Omega \times\left[0, T_{e}\right) .
\end{aligned}
$$

Here, the function $(\hat{v}, \hat{\sigma})$ given as the solution of (19) is considered as known. Next, we show that the problem (22)-(27) has a solution. This will prove the existence of solutions for (4)-(9).

Proof. We will show the existence of solutions using Rothe's method (a timediscretization method, see [Roubíček 2005] for details). In order to introduce a time-discretized problem, let us fix any $m \in \mathbb{N}$ and set

$$
h:=\frac{T_{e}}{2^{m}}, \quad p_{m}^{0}:=0, \quad \hat{\sigma}_{m}^{n}:=\frac{1}{h} \int_{(n-1) h}^{n h} \hat{\sigma}(s) d s \in L^{q}\left(\Omega, \mathbb{R}^{3}\right), \quad n=1, \ldots, 2^{m} .
$$

Then we are looking for functions $u_{m}^{n} \in H^{1}\left(\Omega, \mathbb{R}^{3}\right), \sigma_{m}^{n} \in L^{2}\left(\Omega, \mathscr{Y}^{3}\right)$, and $p_{m}^{n} \in$ $Z_{\text {Curl }}^{2}\left(\Omega, M^{3}\right)$ with $p_{m}^{n}(x) \in \mathfrak{s l}(3)$ for a.e. $x \in \Omega$ and

$$
\Sigma_{n, m}^{\operatorname{lin}}:=\sigma_{m}^{n}+\hat{\sigma}_{m}^{n}-C_{1} \operatorname{dev} \operatorname{sym} p_{m}^{n}-\frac{1}{m} p_{m}^{n}-C_{2} \operatorname{Curl} \operatorname{Curl} p_{m}^{n} \in L^{q}\left(\Omega, M^{3}\right)
$$

solving the problem given by

$$
\begin{aligned}
& -\operatorname{div}_{x} \sigma_{m}^{n}(x)=0, \\
& \sigma_{m}^{n}(x)=\mathbb{C}[x]\left(\operatorname{sym}\left(\nabla_{x} u_{m}^{n}(x)-p_{m}^{n}(x)\right)\right)+(\mathbb{C}[x]-\hat{\mathbb{C}})(\hat{\mathbb{C}})^{-1} \hat{\sigma}_{m}^{n}(x), \\
& \frac{p_{m}^{n}(x)-p_{m}^{n-1}(x)}{h} \in g\left(\Sigma_{n, m}^{\operatorname{lin}}(x)\right),
\end{aligned}
$$

together with the boundary conditions

$$
\begin{aligned}
p_{m}^{n}(x) \times n(x) & =0, \quad x \in \partial \Omega, \\
u_{m}^{n}(x) & =0, \quad x \in \partial \Omega .
\end{aligned}
$$

Next, we adopt the reduction technique proposed in [Alber and Chełmiński 2004] to the equations above. Let $\left(u_{m}^{n}, \sigma_{m}^{n}, p_{m}^{n}\right)$ be a solution of the boundary value problem (28)-(32). Equations (28), (29), and (32) form a boundary value problem for the solution $\left(u_{m}^{n}, \sigma_{m}^{n}\right)$ of the problem of linear elasticity. Due to the linearity of 
this problem we can write these components of the solution in the form

$$
\left(u_{m}^{n}, \sigma_{m}^{n}\right)=\left(U_{m}^{n}, \Sigma_{m}^{n}\right)+\left(w_{m}^{n}, \tau_{m}^{n}\right)
$$

with the solution $\left(w_{m}^{n}, \tau_{m}^{n}\right)$ of the Dirichlet boundary value problem (73) for the data $\hat{b}=0, \hat{\varepsilon}_{p}=(\mathbb{C}-\hat{\mathbb{C}})(\hat{\mathbb{C}})^{-1} \hat{\sigma}_{m}^{n}$, and with the solution $\left(U_{m}^{n}, \Sigma_{m}^{n}\right)$ of the problem (73) for the data $\hat{b}=0, \hat{\varepsilon}_{p}=\operatorname{sym}\left(p_{m}^{n}\right)$. We thus obtain

$$
\operatorname{sym}\left(\nabla_{x} u_{m}^{n}\right)-\operatorname{sym}\left(p_{m}^{n}\right)=\left(P_{2}-I\right) \operatorname{sym}\left(p_{m}^{n}\right)+\operatorname{sym}\left(\nabla_{x} w_{m}^{n}\right),
$$

where the operator $P_{2}$ is defined in Definition A.8. We insert this equation into (29) and get that (30) can be rewritten in the form

$$
\begin{aligned}
& \frac{p_{m}^{n}-p_{m}^{n-1}}{h} \in \mathscr{G}\left(-M_{m} p_{m}^{n}-C_{2} \operatorname{Curl} \operatorname{Curl} p_{m}^{n}+\left(\hat{\sigma}_{m}^{n}+\tau_{m}^{n}\right)\right), \\
& p_{m}^{n}(x) \times n(x)=0, \quad x \in \partial \Omega,
\end{aligned}
$$

where

$$
M_{m}:=\left(\mathbb{C} Q_{2}+L\right) \operatorname{sym}+\frac{1}{m} I: L^{2}\left(\Omega, M^{3}\right) \rightarrow L^{2}\left(\Omega, M^{3}\right),
$$

with the Helmholtz projection $Q_{2}$ and the operator $L$ defined by (76). Here $\mathscr{G}$ denotes the canonical extension of $g$. Next, the problem (33) reads

$$
\Psi\left(p_{m}^{n}\right) \ni \hat{\sigma}_{m}^{n}+\tau_{m}^{n},
$$

where

$$
\Psi(v)=\varphi^{-1}\left(\frac{v-p_{m}^{n-1}}{h}\right)+M_{m}(v)+\partial \Phi(v) .
$$

Here, the functional $\Phi: L^{2}\left(\Omega, \mu^{3}\right) \rightarrow \overline{\mathbb{R}}$ is given by

$$
\Phi(v):= \begin{cases}\frac{1}{2} \int_{\Omega}|\operatorname{Curl} v(x)|^{2} d x, & v \in L_{\text {Curl }, 0}^{2}\left(\Omega, M^{3}\right), \\ +\infty, & \text { otherwise. }\end{cases}
$$

That $\Phi$ is a proper convex lower semicontinuous functional and Curl Curl $=\partial \Phi$ is proved in Section 3. Since $M_{m}$ is bounded, self-adjoint, and positive definite (see Corollary A.10 and the definition of $M_{m}$ ), it is maximal monotone by [Barbu 1976, Theorem II.1.3]. The last thing which we have to verify is whether the operator

$$
\Psi=\mathscr{G}^{-1}+M_{m}+\partial \Phi
$$

is maximal monotone. Since $g \in \mathbb{M}\left(\Omega, \mathcal{M}^{3}, q, \alpha, m\right)$, using the boundedness of $M_{m}$ we conclude that the domains of $\mathscr{G}^{-1}$ and $M_{m}$ are equal to the whole space 
$L^{2}\left(\Omega, M^{3}\right)$. Therefore, Theorem 2.5 guarantees that the sum $\mathscr{G}^{-1}+M_{m}+\partial \Phi$ is maximal monotone with

$$
\operatorname{dom}(\Psi)=\operatorname{dom}(\partial \Phi):=Z_{\text {Curl }}^{2}\left(\Omega, M^{3}\right) .
$$

Since $M_{m}$ is coercive in $L^{2}\left(\Omega, M^{3}\right)$, which obviously yields the coercivity of $\Psi$, the operator $\Psi$ is surjective by Theorem 2.6. Thus, we conclude that (35), as well as the problem (33), has the solutions with the required regularity, that is,

$$
p_{m}^{n} \in Z_{\text {Curl }}^{2}\left(\Omega, M^{3}\right) .
$$

By the constructions this implies that the boundary value problem (28)-(32) is solvable as well (for more details we refer the reader to [Alber and Chełmiński 2004]). Moreover, $p_{m}^{n}(x) \in \mathfrak{s l}(3)$ for a.e. $x \in \Omega$.

Rothe approximation functions. For any family $\left\{\xi_{m}^{n}\right\}_{n=0, \ldots, 2 m}$ of functions in a reflexive Banach space $X$, we define the piecewise affine interpolant $\xi_{m} \in C\left(\left[0, T_{e}\right], X\right)$ by

$$
\xi_{m}(t):=\left(\frac{t}{h}-(n-1)\right) \xi_{m}^{n}+\left(n-\frac{t}{h}\right) \xi_{m}^{n-1} \quad \text { for }(n-1) h \leq t \leq n h,
$$

and the piecewise constant interpolant $\bar{\xi}_{m} \in L^{\infty}\left(0, T_{e} ; X\right)$ by

$$
\bar{\xi}_{m}(t):=\xi_{m}^{n} \quad \text { for }(n-1) h<t \leq n h, n=1, \ldots, 2^{m} \text {, and } \bar{\xi}_{m}(0):=\xi_{m}^{0} .
$$

For further analysis we recall the following property of $\bar{\xi}_{m}$ and $\xi_{m}$ :

$$
\left\|\xi_{m}\right\|_{L^{q}\left(0, T_{e} ; X\right)} \leq\left\|\bar{\xi}_{m}\right\|_{L^{q}\left(-h, T_{e} ; X\right)} \leq\left(h\left\|\xi_{m}^{0}\right\|_{X}^{q}+\left\|\bar{\xi}_{m}\right\|_{L^{q}\left(0, T_{e} ; X\right)}^{q}\right)^{1 / q}
$$

where $\bar{\xi}_{m}$ is formally extended to $t \leq 0$ by $\xi_{m}^{0}$ and $1 \leq q \leq \infty$; see [Roubíček 2005]. A priori estimates. Multiplying (28) by $\frac{u_{m}^{n}-u_{m}^{n-1}}{h}$ and integrating over $\Omega$ we get

$$
\left(\sigma_{m}^{n}, \operatorname{sym}\left(\nabla_{x}\left(u_{m}^{n}-u_{m}^{n-1}\right) / h\right)\right)_{\Omega}=0 .
$$

Equations (29) and (30) imply that for a.e. $x \in \Omega$

$$
\begin{aligned}
& \sigma_{m}^{n} \cdot\left(\operatorname{sym}\left(\nabla_{x}\left(u_{m}^{n}-u_{m}^{n-1}\right) / h\right)-\mathbb{C}^{-1}[x]\left(\sigma_{m}^{n}-\sigma_{m}^{n-1}\right) / h\right) \\
& +\sigma_{m}^{n} \cdot\left(\mathbb{C}^{-1}[x](\mathbb{C}[x]-\hat{\mathbb{C}})(\hat{\mathbb{C}})^{-1}\left(\hat{\sigma}_{m}^{n}-\hat{\sigma}_{m}^{n-1}\right) / h\right) \\
& -\frac{p_{m}^{n}-p_{m}^{n-1}}{h} \cdot\left(C_{1} \operatorname{dev} \operatorname{sym} p_{m}^{n}+\frac{1}{m} p_{m}^{n}+C_{2} \operatorname{Curl} \operatorname{Curl} p_{m}^{n}\right)+\frac{p_{m}^{n}-p_{m}^{n-1}}{h} \cdot \hat{\sigma}_{m}^{n} \\
& =g^{-1}\left(\frac{p_{m}^{n}-p_{m}^{n-1}}{h}\right) \cdot\left(\frac{p_{m}^{n}-p_{m}^{n-1}}{h}\right) \text {. }
\end{aligned}
$$

After integrating the last identity over $\Omega$, the above computations imply 


$$
\begin{aligned}
& \frac{1}{h}\left(\mathbb{C}^{-1} \sigma_{m}^{n}, \sigma_{m}^{n}-\sigma_{m}^{n-1}\right)_{\Omega}+C_{1} \frac{1}{h}\left(\operatorname{dev} \operatorname{sym}\left(p_{m}^{n}-p_{m}^{n-1}\right), \operatorname{dev} \operatorname{sym} p_{m}^{n}\right)_{\Omega} \\
& +\frac{1}{m} \frac{1}{h}\left(p_{m}^{n}-p_{m}^{n-1}, p_{m}^{n}\right)_{\Omega}+C_{2} \frac{1}{h}\left(\operatorname{Curl}\left(p_{m}^{n}-p_{m}^{n-1}\right), \operatorname{Curl} p_{m}^{n}\right)_{\Omega} \\
& +\frac{\alpha}{q}\left\|\Sigma_{n, m}^{\operatorname{lin}}\right\|_{q}^{q}+\frac{\alpha}{q^{*}}\left\|\frac{p_{m}^{n}-p_{m}^{n-1}}{h}\right\|_{q^{*}}^{q^{*}} \\
& \quad \leq \int_{\Omega} m(x) d x+\frac{1}{h}\left(\sigma_{m}^{n}, \overline{\mathbb{C}}\left(\hat{\sigma}_{m}^{n}-\hat{\sigma}_{m}^{n-1}\right)\right)_{\Omega}+\frac{1}{h}\left(\hat{\sigma}_{m}^{n}, p_{m}^{n}-p_{m}^{n-1}\right)_{\Omega},
\end{aligned}
$$

where $\overline{\mathbb{C}}:=\mathbb{C}^{-1}(\mathbb{C}-\hat{\mathbb{C}})(\hat{\mathbb{C}})^{-1}$. Multiplying by $h$ and summing the obtained relation for $n=1, \ldots, l$ for any fixed $l \in\left[1,2^{m}\right]$ we derive the following inequality (here $\left.\mathbb{B}:=\mathbb{C}^{-1}\right)$ :

$$
\begin{aligned}
\frac{1}{2}\left(\left\|\mathbb{B}^{1 / 2} \sigma_{m}^{l}\right\|_{2}^{2}+C_{1}\left\|\operatorname{dev} \operatorname{sym} p_{m}^{l}\right\|_{2}^{2}+\frac{1}{m}\left\|p_{m}^{l}\right\|_{2}^{2}+C_{2}\left\|\operatorname{Curl} p_{m}^{l}\right\|_{2}^{2}\right) \\
+\frac{h \alpha}{q} \sum_{n=1}^{l}\left\|\Sigma_{n, m}^{\operatorname{lin}}\right\|_{q}^{q}+\frac{h \alpha}{q^{*}} \sum_{n=1}^{l}\left\|\frac{p_{m}^{n}-p_{m}^{n-1}}{h}\right\|_{q^{*}}^{q^{*}} \leq C^{(0)}+\int_{\Omega} m(x) d x \\
+h \sum_{n=1}^{l}\left(\sigma_{m}^{n}, \overline{\mathbb{C}} \frac{\hat{\sigma}_{m}^{n}-\hat{\sigma}_{m}^{n-1}}{h}\right)_{\Omega}+h \sum_{n=1}^{l}\left(\hat{\sigma}_{m}^{n}, \frac{p_{m}^{n}-p_{m}^{n-1}}{h}\right)_{\Omega},
\end{aligned}
$$

where ${ }^{5}$

$$
2 C^{(0)}:=\left\|\mathbb{B}^{1 / 2} \sigma^{(0)}\right\|_{2}^{2} .
$$

Since $\hat{\sigma}_{m}^{n} \in L^{q}\left(\Omega, \mathscr{Y}^{3}\right)$, using Young's inequality with $\epsilon>0$ we get that

$$
\begin{aligned}
\left(\hat{\sigma}_{m}^{n}, \frac{p_{m}^{n}-p_{m}^{n-1}}{h}\right)_{\Omega} & \\
& \leq\left\|\hat{\sigma}_{m}^{n}\right\|_{q}\left\|\frac{p_{m}^{n}-p_{m}^{n-1}}{h}\right\|_{q^{*}} \leq C_{\epsilon}\left\|\hat{\sigma}_{m}^{n}\right\|_{q}^{q}+\epsilon\left\|\frac{p_{m}^{n}-p_{m}^{n-1}}{h}\right\|_{q^{*}}^{q^{*}},
\end{aligned}
$$

where $C_{\epsilon}$ is a positive constant appearing in the Young inequality. Analogically, we obtain

$$
\left(\sigma_{m}^{n}, \overline{\mathbb{C}} \frac{\hat{\sigma}_{m}^{n}-\hat{\sigma}_{m}^{n-1}}{h}\right)_{\Omega} \leq \epsilon\left\|\sigma_{m}^{n}\right\|_{2}^{2}+C_{\epsilon}\left\|\frac{\hat{\sigma}_{m}^{n}-\hat{\sigma}_{m}^{n-1}}{h}\right\|_{2}^{2},
$$

${ }^{5}$ Here we use the inequality

$\sum_{n=1}^{l}\left(\phi_{m}^{n}-\phi_{m}^{n-1}, \phi_{m}^{n}\right)_{\Omega}=\frac{1}{2} \sum_{n=1}^{l}\left(\left\|\phi_{m}^{n}\right\|_{2}^{2}-\left\|\phi_{m}^{n-1}\right\|_{2}^{2}\right)+\frac{1}{2} \sum_{n=1}^{l}\left\|\phi_{m}^{n}-\phi_{m}^{n-1}\right\|_{2}^{2} \geq \frac{1}{2}\left\|\phi_{m}^{l}\right\|_{2}^{2}-\frac{1}{2}\left\|\phi_{m}^{0}\right\|_{2}^{2}$,

valid for any family of functions $\phi_{m}^{0}, \phi_{m}^{1}, \ldots, \phi_{m}^{m}$. 
with some other constant $C_{\epsilon}$. Combining inequalities (39), (40), and (41), and choosing an appropriate value for $\epsilon>0$, we obtain the estimate

$$
\begin{aligned}
& \frac{1}{2}\left(\left\|\mathbb{B}^{1 / 2} \sigma_{m}^{l}\right\|_{2}^{2}+C_{1}\left\|\operatorname{dev} \operatorname{sym} p_{m}^{l}\right\|_{2}^{2}\right.\left.+\frac{1}{m}\left\|p_{m}^{l}\right\|_{2}^{2}+C_{2}\left\|\operatorname{Curl} p_{m}^{l}\right\|_{2}^{2}\right) \\
&+h \hat{C}_{\epsilon} \sum_{n=1}^{l}\left(\left\|\Sigma_{n, m}^{\operatorname{lin}}\right\|_{q}^{q}+\left\|\frac{p_{m}^{n}-p_{m}^{n-1}}{h}\right\|_{q^{*}}^{q^{*}}\right) \\
& \leq C^{(0)}+\int_{\Omega} m(x) d x+h \epsilon \sum_{n=1}^{l}\left\|\sigma_{m}^{n}\right\|_{2}^{2}+h \tilde{C}_{\epsilon} \sum_{n=1}^{l}\left(\left\|\hat{\sigma}_{m}^{n}\right\|_{q}^{q}+\left\|\frac{\hat{\sigma}_{m}^{n}-\hat{\sigma}_{m}^{n-1}}{h}\right\|_{2}^{2}\right),
\end{aligned}
$$

where $\tilde{C}, \tilde{C}_{\epsilon}$, and $\hat{C}_{\epsilon}$ are some positive constants. Now, taking [Roubíček 2005, Remark 8.15] and the definition of Rothe's approximation functions into account we rewrite (42) as follows:

$$
\begin{aligned}
& \left\|\mathbb{B}^{1 / 2} \bar{\sigma}_{m}(t)\right\|_{2}^{2}+C_{1}\left\|\operatorname{dev} \operatorname{sym} \bar{p}_{m}(t)\right\|_{2}^{2}+\frac{1}{m}\left\|\bar{p}_{m}(t)\right\|_{2}^{2}+C_{2}\left\|\operatorname{Curl} \bar{p}_{m}(t)\right\|_{2}^{2} \\
& +2 \hat{C}_{\epsilon} \int_{0}^{T_{e}} \int_{\Omega}\left(\left|\partial_{t} p_{m}(x, t)\right|^{q^{*}}+\left|\bar{\Sigma}_{m}^{\operatorname{lin}}(x, t)\right|^{q}\right) d x d t \\
& \quad \leq 2 C^{(0)}+\|m\|_{1, \Omega}+\epsilon\left\|\sigma_{m}\right\|_{2, \Omega \times\left(0, T_{e}\right)}^{2}+2 \tilde{C}_{\epsilon}\|\hat{\sigma}\|_{W^{1, q}\left(0, T_{e} ; L^{q}\left(\Omega, \varphi^{3}\right)\right)}^{q} .
\end{aligned}
$$

From (43) we get immediately that

$$
\begin{aligned}
\bar{C}_{\epsilon}\left\|\sigma_{m}\right\|_{2, \Omega \times(0, t)}^{2}+C_{1} \| \operatorname{dev} \operatorname{sym} & \bar{p}_{m}(t)\left\|_{2}^{2}+\frac{1}{m}\right\| \bar{p}_{m}(t)\left\|_{2}^{2}+C_{2}\right\| \operatorname{Curl} \bar{p}_{m}(t) \|_{2}^{2} \\
+2 \hat{C}_{\epsilon}\left(\left\|\partial_{t} p_{m}\right\|_{q^{*}, \Omega \times\left(0, T_{e}\right)}^{q^{*}}\right. & \left.+\left\|\bar{\Sigma}_{m}^{\operatorname{lin}}\right\|_{q, \Omega \times\left(0, T_{e}\right)}^{q}\right) \\
& \leq 2 C^{(0)}+\|m\|_{1, \Omega}+2 \tilde{C}_{\epsilon}\|\hat{\sigma}\|_{W^{1, q}\left(0, T_{e} ; L^{q}\left(\Omega, \varphi^{3}\right)\right)}^{q},
\end{aligned}
$$

where $\bar{C}_{\epsilon}$ is some other constant depending on $\epsilon$. Altogether, from estimate (44) we get that

$$
\left\{p_{m}\right\}_{m} \text { is uniformly bounded in } W^{1, q^{*}}\left(0, T_{e} ; L^{q^{*}}\left(\Omega, \mathcal{M}^{3}\right)\right),
$$

$\left\{\operatorname{dev} \operatorname{sym} \bar{p}_{m}\right\}_{m}$ is uniformly bounded in $L^{\infty}\left(0, T_{e} ; L^{2}\left(\Omega, M^{3}\right)\right)$,

$$
\left\{\sigma_{m}\right\}_{m} \text {, is uniformly bounded in } L^{2}\left(0, T_{e} ; L^{2}\left(\Omega, \varphi^{3}\right)\right) \text {, }
$$

$\left\{\operatorname{Curl} \bar{p}_{m}\right\}_{m}$ is uniformly bounded in $L^{\infty}\left(0, T_{e} ; L^{2}\left(\Omega, M^{3}\right)\right)$,

$\left\{\bar{\Sigma}_{m}^{\operatorname{lin}}\right\}_{m}$ is uniformly bounded in $L^{q}\left(0, T_{e} ; L^{q}\left(\Omega, M^{3}\right)\right)$,

$$
\left\{\frac{1}{\sqrt{m}} \bar{p}_{m}\right\}_{m} \text { is uniformly bounded in } L^{\infty}\left(0, T_{e} ; L^{2}\left(\Omega, M^{3}\right)\right) \text {. }
$$

In particular, the uniform boundedness of the sequences in (45)-(50) yields 


$$
\left\{u_{m}\right\}_{m} \text { is uniformly bounded in } W^{1, q^{*}}\left(0, T_{e} ; W_{0}^{1, q^{*}}\left(\Omega, \mathbb{R}^{3}\right)\right) \text {, }
$$

$\left\{\operatorname{Curl} \operatorname{Curl} \bar{p}_{m}\right\}_{m}$ is uniformly bounded in $L^{2}\left(0, T_{e} ; L^{2}\left(\Omega, M^{3}\right)\right)$.

In view of (38), the estimates (45)-(52) further imply that the sequences $\left\{\sigma_{m}\right\}_{m}$, $\left\{\operatorname{dev} \operatorname{sym} p_{m}\right\}_{m},\left\{\operatorname{Curl} p_{m}\right\}_{m},\left\{p_{m} / \sqrt{m}\right\}_{m},\left\{\Sigma_{m}^{\operatorname{lin}}\right\}_{m}$, and $\left\{\text { Curl Curl } p_{m}\right\}_{m}$ are also uniformly bounded in the corresponding spaces. As a result, we have

$$
\left\{p_{m}\right\}_{m} \text { is uniformly bounded in } L^{q^{*}}\left(0, T_{e} ; Z_{\text {Curl }}^{q^{*}}\left(\Omega, M^{3}\right)\right) .
$$

Furthermore, due to (3), (46), (48), and (52) we obtain that

$$
\left\{\bar{p}_{m}\right\}_{m} \text { and }\left\{p_{m}\right\}_{m} \text { are uniformly bounded in } L^{2}\left(0, T_{e} ; Z_{\text {Curl }}^{2}\left(\Omega, M^{3}\right)\right) .
$$

Moreover, (36) and (37) yield $\left\{p_{m}(x, t), \bar{p}_{m}(x, t)\right\}_{m} \in \mathfrak{s l}(3)$ for a.e. $(x, t) \in \Omega_{T_{e}}$.

Additional regularity of discrete solutions. In order to get the additional a priori estimates, we extend the function $b$ to $t<0$ by setting $b(t)=b(0)$. The extended function $b$ is in the space $W^{1, p}\left(-2 h, T_{e} ; W^{-1, p}\left(\Omega, \mathbb{R}^{3}\right)\right)$. Then, we set $b_{m}^{0}=b_{m}^{-1}:=$ $b(0)$. Let us further set

$$
p_{m}^{-1}:=p_{m}^{0}-h \mathscr{G}\left(\Sigma_{0, m}^{\operatorname{lin}}\right) .
$$

The assumption (18) implies that $p_{m}^{-1}=0$. Next, we define functions $\left(u_{m}^{-1}, \sigma_{m}^{-1}\right)$ and $\left(u_{m}^{0}, \sigma_{m}^{0}\right)$ as solutions of the linear elasticity problem (73) to the data $\hat{b}=b_{m}^{-1}$, $\hat{\gamma}=0$, and $\hat{\varepsilon}_{p}=0$ and $\hat{b}=b_{m}^{0}, \hat{\gamma}=0$, and $\hat{\varepsilon}_{p}=0$, respectively. Obviously, the following estimate holds:

$$
\left\{\left\|\frac{u_{m}^{0}-u_{m}^{-1}}{h}\right\|_{2},\left\|\frac{\sigma_{m}^{0}-\sigma_{m}^{-1}}{h}\right\|_{2}\right\} \leq C,
$$

where $C$ is some positive constant independent of $m$. Taking now the incremental ratio of (30) for $n=1, \ldots, 2^{m}$, we obtain ${ }^{6}$

$$
\text { rt } p_{m}^{n}-\mathrm{rt} p_{m}^{n-1}=\mathscr{G}\left(\Sigma_{n, m}^{\operatorname{lin}}\right)-\mathscr{G}\left(\Sigma_{(n-1), m}^{\operatorname{lin}}\right) .
$$

Let us now multiply the last identity by $-\left(\Sigma_{n, m}^{\operatorname{lin}}-\Sigma_{(n-1), m}^{\operatorname{lin}}\right) / h$. Then, using the monotonicity of $\mathscr{G}$ we obtain

$$
\begin{aligned}
& \frac{1}{m}\left(\mathrm{rt} p_{m}^{n}-\mathrm{rt} p_{m}^{n-1}, \mathrm{rt} p_{m}^{n}\right)_{\Omega} \\
& +\left(\mathrm{rt} p_{m}^{n}-\mathrm{rt} p_{m}^{n-1}, \operatorname{dev} \operatorname{sym}\left(\mathrm{rt} p_{m}^{n}\right)\right)_{\Omega}+\left(\mathrm{rt} p_{m}^{n}-\mathrm{rt} p_{m}^{n-1}, \operatorname{CurlCurl}\left(\mathrm{rt} p_{m}^{n}\right)\right)_{\Omega} \\
& \quad \leq\left(\mathrm{rt} p_{m}^{n}-\mathrm{rt} p_{m}^{n-1}, \mathrm{rt} \sigma_{m}^{n}\right)_{\Omega}+\left(\mathrm{rt} p_{m}^{n}-\mathrm{rt} p_{m}^{n-1}, \mathrm{rt} \hat{\sigma}_{m}^{n}\right)_{\Omega} .
\end{aligned}
$$

${ }^{6}$ For simplicity we use the notation $\operatorname{rt} \phi_{m}^{n}:=\left(\phi_{m}^{n}-\phi_{m}^{n-1}\right) / h$, where $\phi_{m}^{0}, \phi_{m}^{1}, \ldots, \phi_{m}^{m}$ is any family of functions. 
With (28) and (29) the previous inequality can be rewritten as follows:

$$
\begin{aligned}
& \frac{1}{m}\left(\mathrm{rt} p_{m}^{n}-\mathrm{rt} p_{m}^{n-1}, \mathrm{rt} p_{m}^{n}\right)_{\Omega}+\left(\mathrm{rt} p_{m}^{n}-\mathrm{rt} p_{m}^{n-1}, \operatorname{dev} \operatorname{sym}\left(\mathrm{rt} p_{m}^{n}\right)\right)_{\Omega} \\
& +\left(\operatorname{rt} p_{m}^{n}-\mathrm{rt} p_{m}^{n-1}, \operatorname{Curl} \operatorname{Curl}\left(\operatorname{rt} p_{m}^{n}\right)\right)_{\Omega}+\left(\operatorname{rt} \sigma_{m}^{n}-\mathrm{rt} \sigma_{m}^{n-1}, \mathbb{C}^{-1} \mathrm{rt} \sigma_{m}^{n}\right)_{\Omega} \\
& \leq\left(\mathrm{rt} \hat{\sigma}_{m}^{n}-\mathrm{rt} \hat{\sigma}_{m}^{n-1}, \overline{\mathbb{C}} \mathrm{rt} \sigma_{m}^{n}\right)_{\Omega}+\left(\mathrm{rt} p_{m}^{n}-\mathrm{rt} p_{m}^{n-1}, \mathrm{rt} \hat{\sigma}_{m}^{n}\right)_{\Omega} \text {. }
\end{aligned}
$$

As in the proof of (39), multiplying the last inequality by $h$ and summing with respect to $n$ from 1 to $l$ for any fixed $l \in\left[1,2^{m}\right]$ we get the estimate

$$
\begin{aligned}
& \frac{h}{m}\left\|\mathrm{rt} p_{m}^{l}\right\|_{2}^{2}+h \| \operatorname{dev} \text { sym rt } p_{m}^{l}\left\|_{2}^{2}+h\right\| \mathbb{B}^{1 / 2} \mathrm{rt} \sigma_{m}^{l}\left\|_{2}^{2}+h\right\| \operatorname{Curl} \mathrm{rt} p_{m}^{l} \|_{2}^{2} \\
& \leq 2 h C^{(0)}+2 h \sum_{n=1}^{l}\left(\mathrm{rt} \hat{\sigma}_{m}^{n}, \mathrm{rt} p_{m}^{n}-\mathrm{rt} p_{m}^{n-1}\right)_{\Omega}+2 h \sum_{n=1}^{l}\left(\mathrm{rt} \hat{\sigma}_{m}^{n}-\mathrm{rt} \hat{\sigma}_{m}^{n-1}, \overline{\mathbb{C}} \mathrm{rt} \sigma_{m}^{n}\right)_{\Omega},
\end{aligned}
$$

where now $C^{(0)}$ is defined by

$$
2 C^{(0)}:=\left\|\mathbb{B}^{1 / 2} \mathrm{rt} \sigma_{m}^{0}\right\|_{2}^{2} .
$$

We note that (55) yields the uniform boundness of $C^{(0)}$ with respect to $m$. Summing now (56) for $l=1, \ldots, 2^{m}$ we derive the inequality

$$
\begin{aligned}
\frac{1}{m}\left\|\partial_{t} p_{m}\right\|_{2, \Omega_{T_{e}}}^{2} & +\left\|\operatorname{dev} \operatorname{sym}\left(\partial_{t} p_{m}\right)\right\|_{2, \Omega_{T_{e}}}^{2}+\left\|\operatorname{Curl}\left(\partial_{t} p_{m}\right)\right\|_{2, \Omega_{T_{e}}}^{2} \\
& +C\left\|\partial_{t} \sigma_{m}\right\|_{2, \Omega_{T_{e}}}^{2} \leq C\left\|\partial_{t} \hat{\sigma}_{m}\right\|_{2, \Omega_{T_{e}}}\left(\left\|\partial_{t} \sigma_{m}\right\|_{2, \Omega_{T_{e}}}+\left\|\partial_{t} p_{m}\right\|_{2, \Omega_{T_{e}}}\right) .
\end{aligned}
$$

Using now inequality (3), the condition $\partial_{t} p_{m}(x, t) \in \mathfrak{s l}(3)$ for a.e. $(x, t) \in \Omega_{T_{e}}$, and Young's inequality with $\epsilon>0$ in (57), we obtain that

$$
\frac{1}{m}\left\|\partial_{t} p_{m}\right\|_{2, \Omega_{T_{e}}}^{2}+C_{\epsilon}\left\|\partial_{t} p_{m}\right\|_{2, \Omega_{T_{e}}}^{2}+C_{\epsilon}\left\|\partial_{t} \sigma_{m}\right\|_{2, \Omega_{T_{e}}}^{2} \leq C\left\|\partial_{t} \hat{\sigma}_{m}\right\|_{2, \Omega_{T_{e}}}^{2} .
$$

Since $\hat{\sigma}_{m}$ is uniformly bounded in $W^{1, q}\left(\Omega_{T_{e}}, \mathscr{S}^{3}\right)$, estimates (57) and (58) imply

$\left\{\operatorname{dev} \operatorname{sym} \partial_{t} p_{m}\right\}_{m}$ is uniformly bounded in $L^{2}\left(0, T_{e} ; L^{2}\left(\Omega, M^{3}\right)\right)$,

$\left\{\partial_{t} \sigma_{m}\right\}_{m}$ is uniformly bounded in $L^{2}\left(0, T_{e} ; L^{2}\left(\Omega, M^{3}\right)\right)$,

$\left\{\text { Curl } \partial_{t} p_{m}\right\}_{m}$ is uniformly bounded in $L^{2}\left(0, T_{e} ; L^{2}\left(\Omega, M^{3}\right)\right)$,

$\left\{\frac{1}{\sqrt{m}} \partial_{t} p_{m}\right\}_{m}$ is uniformly bounded in $L^{2}\left(0, T_{e} ; L^{2}\left(\Omega, M^{3}\right)\right)$,

$$
\left\{p_{m}\right\}_{m} \text { is uniformly bounded in } H^{1}\left(0, T_{e} ; L_{\text {Curl }}^{2}\left(\Omega, \mathcal{M}^{3}\right)\right) \text {. }
$$

Existence of solutions. By estimates (45)-(54) and (59)-(63) and at the expense of extracting a subsequence, we have that the sequences in (45)-(54) and (59)-(63) converge with respect to weak and weak-star topologies in corresponding spaces, 
respectively. Next, we claim that weak limits of $\left\{\bar{p}_{m}\right\}_{m}$ and $\left\{p_{m}\right\}_{m}$ coincide. Indeed, using (45) this can be shown as follows:

$$
\begin{aligned}
\left\|p_{m}-\bar{p}_{m}\right\|_{\Omega_{T_{e}}}^{2} & =\sum_{n=1}^{m} \int_{(n-1) h}^{n h}\left\|\left(p_{m}^{n}-p_{m}^{n-1}\right) \frac{t-n h}{h}\right\|_{2}^{2} d t \\
& =\frac{h^{2+1}}{2+1} \sum_{n=1}^{m}\left\|\frac{p_{m}^{n}-p_{m}^{n-1}}{h}\right\|_{2}^{2}=\frac{h^{2}}{2+1}\left\|\frac{d p_{m}}{d t}\right\|_{2, \Omega_{T_{e}}}^{2},
\end{aligned}
$$

which implies that $\bar{p}_{m}-p_{m}$ converges strongly to 0 in $L^{2}\left(\Omega_{T_{e}}, M^{3}\right)$. The proof of the fact that the difference $\bar{\sigma}_{m}-\sigma_{m}$ converges weakly to 0 in $L^{2}\left(\Omega_{T_{e}}, \varphi^{3}\right)$ can be performed as on p. 210 of [Roubíček 2005]. For the reader's convenience we reproduce the reasoning used there. Let us choose some appropriate number $d \in$ $\mathbb{N}$ and then fix any integer $n_{0} \in\left[1,2^{d}\right]$. Let $h_{0}=T_{e} / 2^{n_{0}}$. Consider functions $I_{\left[h_{0}\left(n_{0}-1\right), h_{0} n_{0}\right]} v$ with $v \in L^{2}\left(\Omega, \mathscr{Y}^{3}\right)$, where $I_{K}$ denotes the indicator function of a set $K$. We note that, according to Proposition 1.36 of the same reference, the linear combinations of all such functions are dense in $L^{2}\left(\Omega_{T_{e}}, \varphi^{3}\right)$. Then for any $h \leq h_{0}{ }^{7}$ we have

$$
\begin{aligned}
& \left(\sigma_{m}-\bar{\sigma}_{m}, I_{\left[h_{0}\left(n_{0}-1\right), h_{0} n_{0}\right]} v\right)_{\Omega_{T_{e}}} \\
& =\int_{h_{0}\left(n_{0}-1\right)}^{h_{0} n_{0}}\left(\sigma_{m}(t)-\bar{\sigma}_{m}(t), v\right)_{\Omega} d t=\sum_{n=\frac{h_{0}}{h}\left(n_{0}-1\right)+1}^{h_{0} n_{0} / h} \int_{(n-1) h}^{n h}\left(\left(\sigma_{m}^{n}-\sigma_{m}^{n-1}\right) \frac{t-n h}{h}, v\right)_{\Omega} d t \\
& =-\frac{h}{2}\left(\sigma_{m}^{h_{0} n_{0} / h}-\sigma_{m}^{h_{0}\left(n_{0}-1\right) / h}, v\right)_{\Omega}=-\frac{h}{2}\left(\bar{\sigma}_{m}\left(h_{0} n_{0}\right)-\bar{\sigma}_{m}\left(h_{0}\left(n_{0}-1\right)\right), v\right)_{\Omega} .
\end{aligned}
$$

Employing (47) we get that $\bar{\sigma}_{m}-\sigma_{m}$ converges weakly to 0 in $L^{2}\left(\Omega_{T_{e}}, \mathscr{\varphi}^{3}\right)$. Next, by (50) the sequence $\left\{p_{m} / m\right\}_{m}$ converges strongly to 0 in $L^{2}\left(\Omega_{T_{e}}, M^{3}\right)$. Summarizing all observations made above we may conclude that the limit functions denoted by $\tilde{v}, \tilde{\sigma}, p$, and $\Sigma^{\text {lin }}$ have the following properties:

$$
\begin{gathered}
(\tilde{v}, \tilde{\sigma}) \in H^{1}\left(0, T_{e} ; H_{0}^{1}\left(\Omega, \mathbb{R}^{3}\right) \times L^{2}\left(\Omega, \mathscr{S}^{3}\right)\right), \quad \Sigma^{\operatorname{lin}} \in L^{q}\left(\Omega_{T_{e}}, M^{3}\right), \\
p \in H^{1}\left(0, T_{e} ; L_{\text {Curl }}^{2}\left(\Omega, M^{3}\right)\right) \cap L^{2}\left(0, T_{e} ; Z_{\text {Curl }}^{2}\left(\Omega, M^{3}\right)\right) .
\end{gathered}
$$

Moreover, $p(x, t) \in \mathfrak{s l}(3)$ holds for a.e. $(x, t) \in \Omega_{T_{e}}$. Before passing to the weak limit, we note that the Rothe approximation functions satisfy the equations

$$
\begin{aligned}
-\operatorname{div}_{x} \bar{\sigma}_{m}(x, t) & =\bar{b}_{m}(x, t), \\
\sigma_{m}(x, t) & =\mathbb{C}\left(\operatorname{sym}\left(\nabla_{x} u_{m}(x, t)-p_{m}(x, t)\right)\right)+(\mathbb{C}[x]-\hat{\mathbb{C}})(\hat{\mathbb{C}})^{-1} \hat{\sigma}_{m}(x), \\
\partial_{t} p_{m}(x, t) & \in g\left(\bar{\Sigma}_{m}^{\operatorname{lin}}(x, t)\right),
\end{aligned}
$$

${ }^{7}$ We recall that $h$ is chosen to be equal to $T_{e} / 2^{m}$ for some $m \in \mathbb{N}$. 
together with the initial and boundary conditions

$$
\begin{aligned}
p_{m}(x, 0)=0, & x \in \Omega, \\
p_{m}(x, t) \times n(x)=0, & x \in \partial \Omega, \\
u_{m}(x, t)=0, & x \in \partial \Omega .
\end{aligned}
$$

Passing to the weak limit in (64), (65), and (69) we obtain that the limit functions $\tilde{v}, \tilde{\sigma}, p$, and $\Sigma^{\operatorname{lin}}$ satisfy (22) and (27). To show that the limit functions satisfy also (24) we proceed as follows: As above, the system (64)-(69) can be rewritten as

$$
\begin{aligned}
\int_{0}^{T_{e}} & \int_{\Omega}\left(g^{-1}\left(\partial_{t} p_{m}(x, t)\right) \cdot \partial_{t} p_{m}(x, t)\right) d x d t \\
=-( & \left.\frac{d \sigma_{m}}{d t}, \mathbb{C}^{-1} \bar{\sigma}_{m}\right)_{\Omega_{T_{e}}}-C_{1}\left(\frac{d p_{m}}{d t}, \operatorname{dev} \operatorname{sym} \bar{p}_{m}\right)_{\Omega_{T_{e}}}-\frac{1}{m}\left(\frac{d p_{m}}{d t}, \bar{p}_{m}\right)_{\Omega_{T_{e}}} \\
& \quad-C_{2}\left(\frac{d p_{m}}{d t}, \operatorname{Curl} \operatorname{Curl} \bar{p}_{m}\right)_{\Omega_{T_{e}}}+\left(\hat{\sigma}_{m}, \partial_{t} p_{m}\right)_{\Omega_{T_{e}}}+\left(\overline{\mathbb{C}} \bar{\sigma}_{m}, \partial_{t} \hat{\sigma}_{m}\right)_{\Omega_{T_{e}}} .
\end{aligned}
$$

Due to (59)-(63) and Lemma B.11 we can pass to the weak limit inferior in (70) to get the following inequality:

$$
\begin{aligned}
\limsup _{m \rightarrow \infty} \int_{0}^{T_{e}} \int_{\Omega}\left(g^{-1}\left(\partial_{t} p_{m}(x, t)\right) \cdot \partial_{t} p_{m}(x, t)\right) d x d t \\
\leq\left(\partial_{t} p, \tilde{\sigma}+\hat{\sigma}-\operatorname{dev} \operatorname{sym} p-\operatorname{Curl} \text { Curl } p\right)_{\Omega_{T_{e}}} .
\end{aligned}
$$

Let $\mathscr{G}$ denote the canonical extension of $g$. Then (71) reads as follows:

$$
\limsup _{m \rightarrow \infty}\left(\mathscr{G}^{-1}\left(\partial_{t} p_{m}\right), \partial_{t} p_{m}\right)_{\Omega_{T_{e}}} \leq\left(\partial_{t} p, \tilde{\sigma}+\hat{\sigma}-\operatorname{dev} \operatorname{sym} p-\operatorname{Curl} \text { Curl } p\right)_{\Omega_{T_{e}}}
$$

Since $\mathscr{G}^{-1}$ is pseudomonotone, inequality (72) yields that, for a.e. $(x, t) \in \Omega_{T_{e}}$,

$$
\partial_{t} p(x, s) \in g(\tilde{\sigma}(x, t)+\hat{\sigma}(x, t)-\operatorname{dev} \operatorname{sym} p(x, t)-\operatorname{Curl} \operatorname{Curl} p(x, t)) .
$$

Therefore, we conclude that the limit functions $\tilde{v}, \tilde{\sigma}, p$, and $\Sigma^{\text {lin }}$ satisfy (22)-(27) and the existence of strong solutions is herewith established.

This completes the proof of Theorem 4.7.

\section{Appendix A: Helmholtz's projection}

In this section we present some results concerning projection operators to spaces of tensor fields, which are symmetric gradients, and to spaces of tensor fields with vanishing divergence. For details the reader is referred to [Alber and Chełmiński 2007]. 
In linear elasticity theory it is well known (see [Giusti 2003, Theorem 10.15]) that a Dirichlet boundary value problem formed by the equations

$$
\begin{aligned}
-\operatorname{div}_{x} \sigma(x) & =\hat{b}(x), & & x \in \Omega, \\
\sigma(x) & =\mathbb{C}[x]\left(\operatorname{sym}\left(\nabla_{x} u(x)\right)-\hat{\varepsilon}_{p}(x)\right), & & x \in \Omega, \\
u(x) & =0, & & x \in \partial \Omega,
\end{aligned}
$$

for given $\hat{b} \in W^{-1, q}\left(\Omega, \mathbb{R}^{3}\right)$ and $\hat{\varepsilon}_{p} \in L^{q}\left(\Omega, \mathscr{\varphi}^{3}\right)$ has a unique weak solution $(u, \sigma) \in W_{0}^{1, q}\left(\Omega, \mathbb{R}^{3}\right) \times L^{q}\left(\Omega, \mathscr{Y}^{3}\right)$ provided the open set $\Omega$ has a $C^{1}$-boundary and $\mathbb{C} \in C\left(\bar{\Omega}, \mathscr{S}^{3}\right)$. Here the number $q$ satisfies $1<q<\infty$. For $\hat{b}=0$ the solution of (73) satisfies the inequality

$$
\left\|\operatorname{sym}\left(\nabla_{x} u\right)\right\|_{q} \leq C\left\|\hat{\varepsilon}_{p}\right\|_{q}
$$

with some positive constant $C$.

Definition A.8. For every $\hat{\varepsilon}_{p} \in L^{q}\left(\Omega, \mathscr{S}^{3}\right)$ we define a linear operator $P_{q}: L^{q}\left(\Omega, \mathscr{S}^{3}\right)$ $\rightarrow L^{q}\left(\Omega, \mathscr{\varphi}^{3}\right)$ by

$$
P_{q} \hat{\varepsilon}_{p}:=\operatorname{sym}\left(\nabla_{x} u\right),
$$

where $u \in W_{0}^{1, q}\left(\Omega, \mathbb{R}^{3}\right)$ is the unique weak solution of (73) to the given function $\hat{\varepsilon}_{p}$ and $\hat{b}=0$.

Next, a subset $\varphi^{q}$ of $L^{q}\left(\Omega, \mathscr{Y}^{3}\right)$ is defined by

$$
\varphi^{q}=\left\{\operatorname{sym}\left(\nabla_{x} u\right) \mid u \in W_{0}^{1, q}\left(\Omega, \mathbb{R}^{3}\right)\right\} .
$$

The main properties of $P_{q}$ are stated in the following lemma.

Lemma A.9. For every $1<q<\infty$ the operator $P_{p}$ is a bounded projector onto the subset $\mathscr{G}^{q}$ of $L^{q}\left(\Omega, \mathscr{Y}^{3}\right)$. The projector $\left(P_{q}\right)^{*}$ adjoint with respect to the bilinear form $[\xi, \zeta]_{\Omega}:=(\xi, \zeta)_{\Omega}$ on $L^{q}\left(\Omega, \varphi^{3}\right) \times L^{q^{*}}\left(\Omega, \varphi^{3}\right)$ satisfies

$$
\left(P_{q}\right)^{*}=P_{q^{*}}, \quad \text { where } \frac{1}{q^{*}}+\frac{1}{q}=1 .
$$

Due to Lemma A.9 the projection operator

$$
Q_{q}=\left(I-P_{q}\right): L^{q}\left(\Omega, \mathscr{Y}^{3}\right) \rightarrow L^{q}\left(\Omega, \mathscr{Y}^{3}\right)
$$

is well defined and generalizes the classical Helmholtz projection.

Let $L: \varphi^{3} \rightarrow \varphi^{3}$ be the linear, positive semidefinite mapping given by

$$
L v=C_{1} \operatorname{dev} v .
$$

The next result is needed for the subsequent analysis. 
Corollary A.10. Let

$$
\left(\mathbb{C} P_{q}+L\right)^{*}
$$

be the operator adjoint to $\mathbb{C} P_{q}+L: L^{q}\left(\Omega, \mathscr{Y}^{3}\right) \rightarrow L^{q}\left(\Omega, \mathscr{S}^{3}\right)$ with respect to the bilinear form $(\xi, \zeta)_{\Omega}$ on the product space $L^{q}\left(\Omega, \varphi^{3}\right) \times L^{q^{*}}\left(\Omega, \varphi^{3}\right)$. Then $\left(\mathbb{C} P_{q}+L\right)^{*}=\mathbb{C} P_{q^{*}}+L$. Moreover, the operator $\mathbb{C} Q_{2}+L$ is nonnegative and self-adjoint.

For the proof of this result the reader is referred to [Alber and Chełmiński 2004].

\section{Appendix B}

In this appendix we prove the following lemma (see [Roubíček 2005]).

Lemma B.11. Let $X$ be a reflexive Banach space embedded continuously and densely into a Hilbert space $H$, let the functions $\phi_{m}$ and $\bar{\phi}_{m}$ be defined by (36) and (37) for any family of functions $\phi_{m}^{0}, \phi_{m}^{1}, \ldots, \phi_{m}^{m}$, respectively, and let $\phi$ be a weak limit of $\phi_{m}$. Then the following inequality:

$$
\limsup _{m \rightarrow \infty}\left\langle\frac{d \phi_{m}}{d t}, \bar{\phi}_{m}\right\rangle_{L^{q}\left(X^{*}\right), L^{p}(X)} \geq\left\langle\frac{d \phi}{d t}, \phi\right\rangle_{L^{q}\left(X^{*}\right), L^{p}(X)}
$$

holds, where $\langle\cdot, \cdot\rangle_{L^{q}\left(X^{*}\right), L^{p}(X)}$ denotes the dual pairing between $L^{p}(X)$ and $L^{q}\left(X^{*}\right)$. Proof. The last inequality results from the next line by taking lim sup from both sides and using the lower semicontinuity of the norm

$$
\begin{aligned}
\left\langle\frac{d \phi_{m}}{d t},\right. & \left.\bar{\phi}_{m}\right\rangle_{L^{q}\left(X^{*}\right), L^{p}(X)} \\
= & \sum_{n=1}^{m} \int_{h(n-1)}^{h n}\left\langle\frac{\phi_{m}^{n}-\phi_{m}^{n-1}}{h}, \phi_{m}^{n}\right\rangle_{X^{*}, X} d t=\sum_{n=1}^{m}\left\langle\phi_{m}^{n}-\phi_{m}^{n-1}, \phi_{m}^{n}\right\rangle_{X^{*}, X} \\
& =\sum_{n=1}^{m} \frac{1}{2}\left\|\phi_{m}^{n}\right\|_{H}^{2}-\frac{1}{2}\left\|\phi_{m}^{n-1}\right\|_{H}^{2}+\frac{1}{2}\left\|\phi_{m}^{n}-\phi_{m}^{n-1}\right\|_{H}^{2} \geq \frac{1}{2}\left\|\phi_{m}^{m}\right\|_{H}^{2}-\frac{1}{2}\left\|\phi_{m}^{0}\right\|_{H}^{2} .
\end{aligned}
$$

The proof is completed by generalized integration by parts.

\section{References}

[Alber and Chełmiński 2004] H.-D. Alber and K. Chełmiński, "Quasistatic problems in viscoplasticity theory, I: Models with linear hardening”, pp. 105-129 in Operator theoretical methods and applications to mathematical physics, edited by I. Gohberg et al., Operator Theory: Advances and Applications 147, Birkhäuser, Basel, 2004.

[Alber and Chełmiński 2007] H.-D. Alber and K. Chełmiński, "Quasistatic problems in viscoplasticity theory, II: Models with nonlinear hardening”, Math. Models Methods Appl. Sci. 17:2 (2007), 189-213. 
[Barbu 1976] V. Barbu, Nonlinear semigroups and differential equations in Banach spaces, Editura Academiei Republicii Socialiste România, Bucharest, 1976.

[Bardella 2010] L. Bardella, "Size effects in phenomenological strain gradient plasticity constitutively involving the plastic spin”, Int. J. Eng. Sci. 48:5 (2010), 550-568.

[Brézis 1970] H. Brézis, “On some degenerate nonlinear parabolic equations”, pp. 28-38 in Nonlinear functional analysis, I (Chicago, IL, 1968), edited by F. E. Browder, Proceedings of Symposia in Pure Mathematics 18, American Mathematical Society, Providence, RI, 1970.

[Castaing and Valadier 1977] C. Castaing and M. Valadier, Convex analysis and measurable multifunctions, Lecture Notes in Mathematics 580, Springer, Berlin, 1977.

[Damlamian et al. 2007] A. Damlamian, N. Meunier, and J. Van Schaftingen, "Periodic homogenization of monotone multivalued operators", Nonlinear Anal. 67:12 (2007), 3217-3239.

[Ebobisse and Neff 2010] F. Ebobisse and P. Neff, "Existence and uniqueness for rate-independent infinitesimal gradient plasticity with isotropic hardening and plastic spin", Math. Mech. Solids 15:6 (2010), 691-703.

[Giacomini and Lussardi 2008] A. Giacomini and L. Lussardi, "Quasi-static evolution for a model in strain gradient plasticity”, SIAM J. Math. Anal. 40:3 (2008), 1201-1245.

[Giusti 2003] E. Giusti, Direct methods in the calculus of variations, World Scientific, River Edge, NJ, 2003.

[Gurtin and Anand 2005] M. E. Gurtin and L. Anand, "A theory of strain-gradient plasticity for isotropic, plastically irrotational materials, I: Small deformations", J. Mech. Phys. Solids 53:7 (2005), 1624-1649.

[Gurtin and Needleman 2005] M. E. Gurtin and A. Needleman, "Boundary conditions in smalldeformation, single-crystal plasticity that account for the Burgers vector", J. Mech. Phys. Solids 53:1 (2005), 1-31.

[Hu and Papageorgiou 1997] S. Hu and N. S. Papageorgiou, Handbook of multivalued analysis, I: Theory, Mathematics and its Applications 419, Kluwer, Dordrecht, 1997.

[Kato 1966] T. Kato, Perturbation theory for linear operators, Grundlehren der mathematischen Wissenschaften 132, Springer, Berlin, 1966.

[Kratochvíl et al. 2010] J. Kratochvíl, M. Kružík, and R. Sedláček, "Energetic approach to gradient plasticity", Z. Angew. Math. Mech. 90:2 (2010), 122-135.

[Mainik and Mielke 2009] A. Mainik and A. Mielke, "Global existence for rate-independent gradient plasticity at finite strain”, J. Nonlinear Sci. 19:3 (2009), 221-248.

[Menzel and Steinmann 2000] A. Menzel and P. Steinmann, "On the continuum formulation of higher gradient plasticity for single and polycrystals", J. Mech. Phys. Solids 48:8 (2000), 17771796. Erratum in 49:5, (2001), 1179-1180.

[Neff 2008a] P. Neff, "Remarks on invariant modelling in finite strain gradient plasticity", Tech. Mech. 28:1 (2008), 13-21.

[Neff 2008b] P. Neff, "Uniqueness of strong solutions in infinitesimal perfect gradient plasticity with plastic spin”, pp. 129-140 in IUTAM Symposium on Theoretical, Computational and Modelling Aspects of Inelastic Media (Cape Town, 2008), edited by B. D. Reddy, IUTAM Bookseries 11, Springer, Berlin, 2008.

[Neff et al. 2009a] P. Neff, K. Chełmiński, and H.-D. Alber, "Notes on strain gradient plasticity: finite strain covariant modelling and global existence in the infinitesimal rate-independent case", Math. Models Methods Appl. Sci. 19:2 (2009), 307-346. 
[Neff et al. 2009b] P. Neff, A. Sydow, and C. Wieners, "Numerical approximation of incremental infinitesimal gradient plasticity”, Int. J. Numer. Methods Eng. 77:3 (2009), 414-436.

[Neff et al. 2011] P. Neff, D. Pauly, and K.-J. Witsch, "A canonical extension of Korn's first inequality to $H$ (Curl) motivated by gradient plasticity with plastic spin”, C. R. Acad. Sci. Paris Sér. I Math. 349:23-24 (2011), 1251-1254.

[Neff et al. 2012a] P. Neff, D. Pauly, and K.-J. Witsch, "Maxwell meets Korn: a new coercive inequality for tensor fields in $\mathbb{R}^{N \times N}$ with square-integrable exterior derivative", Math. Methods Appl. Sci. 35:1 (2012), 65-71.

[Neff et al. 2012b] P. Neff, D. Pauly, and K.-J. Witsch, “On a canonical extension of Korn's first and Poincaré's inequalities to H(Curl)", J. Math. Sci. (NY) 185:5 (2012), 721-727.

[Neff et al. 2012c] P. Neff, D. Pauly, and K.-J. Witsch, "Poincaré meets Korn via Maxwell: extending Korn's first inequality to incompatible tensor fields", preprint SM-E-753, Universität DuisburgEssen, Fakultät für Mathematik, 2012. arXiv 1203.2744

[Nesenenko and Neff 2012] S. Nesenenko and P. Neff, "Well-posedness for dislocation based gradient visco-plasticity, I: Subdifferential case”, SIAM J. Math. Anal. 44:3 (2012), 1694-1712.

[Pankov 1997] A. Pankov, G-convergence and homogenization of nonlinear partial differential operators, Mathematics and its Applications 422, Kluwer, Dordrecht, 1997.

[Pascali and Sburlan 1978] D. Pascali and S. Sburlan, Nonlinear mappings of monotone type, Martinus Nijhoff, The Hague, 1978.

[Phelps 1993] R. R. Phelps, Convex functions, monotone operators and differentiability, 2nd ed., Lecture Notes in Mathematics 1364, Springer, Berlin, 1993.

[Reddy et al. 2008] B. D. Reddy, F. Ebobisse, and A. T. McBride, "Well-posedness of a model of strain gradient plasticity for plastically irrotational materials.”, Int. J. Plast. 24:1 (2008), 55-73.

[Roubíček 2005] T. Roubíček, Nonlinear partial differential equations with applications, International Series of Numerical Mathematics 153, Birkhäuser, Basel, 2005.

[Simons 1998] S. Simons, Minimax and monotonicity, Lecture Notes in Mathematics 1693, Springer, Berlin, 1998.

[Sohr 2001] H. Sohr, The Navier-Stokes equations: an elementary functional analytic approach, Birkhäuser, Basel, 2001.

[Svendsen et al. 2009] B. Svendsen, P. Neff, and A. Menzel, "On constitutive and configurational aspects of models for gradient continua with microstructure”, Z. Angew. Math. Mech. 89:8 (2009), 687-697.

Received 30 Apr 2012. Revised 5 Sep 2012. Accepted 20 Oct 2012.

SERGIY NESENENKO: nesenenko@mathematik.tu-darmstadt.de

Fachbereich Mathematik, Technische Universität Darmstadt, Schlossgartenstrasse 7,

D-64289 Darmstadt, Germany

PATRIZIO NeFF: patrizio.neff@uni-due.de

Fakultät für Mathematik, Universität Duisburg-Essen, Campus Essen, Universitätsstrasse 2,

D-45141 Essen, Germany 


\title{
SYMMETRY CLASSES FOR EVEN-ORDER TENSORS
}

\author{
Marc Olive AND Nicolas AufFray
}

\begin{abstract}
We give a complete general answer to the problem, recurrent in continuum mechanics, of determining of the number and type of symmetry classes of an even-order tensor space. This kind of investigation was initiated for the space of elasticity tensors, and since then different authors have solved this problem for other kinds of physics, such as photoelectricity, piezoelectricity, flexoelectricity, and strain-gradient elasticity. All these problems were treated using the same computational method, which, though effective, has the drawback of not providing general results. Furthermore, its complexity increases with the tensorial order. Here we provide general theorems that directly give the desired results for any even-order constitutive tensor. As an illustration of this method, and for the first time, the symmetry classes of all even-order tensors of Mindlin second strain-gradient elasticity are provided.
\end{abstract}

\section{Introduction}

Physical motivation. In the last years there has been increased interest in generalized continuum theories; see, for example, [Forest 1998; dell'Isola et al. 2009; 2012; Lebée and Sab 2011]. These works, based on the pioneering articles [Toupin 1962; Mindlin 1964; 1965], propose extended kinematic formulations, to take into account size effects within the continuum. The price to be paid for this is the appearance of tensors of order greater than four in the constitutive relations. These higher-order objects are difficult to handle and extracting physically meaningful information from them is not straightforward. The aim of this paper is to provide general results concerning the type and number of anisotropic systems an evenorder tensor can have.

Such results have important applications, at least, for the modeling and numerical implementation of nonclassical linear constitutive laws:

Modeling: The purpose of modeling is, given a material and a set of physical variables of interest, to construct the more general constitutive law (a linear one, in the present context) that describes the behavior of that material. An example of such a method is provided in [Thionnet and Martin 2006], where,

MSC2010: 15A72, 20C35, 74B99.

Keywords: anisotropy, symmetry classes, higher-order tensors, generalized continuum theories, strain-gradient elasticity. 
given a set of variables $V$ and the material symmetry group $S$, the authors derive mechanical behavior laws using the theory of invariants and continuum thermodynamics. In such regard our results will say, without making any computation whether or not $S$ is contained in the set of symmetry classes of $\mathscr{L}\left(v, v^{\prime}\right)$ the space of linear maps from $v \in V$ to $v^{\prime} \in V$.

Numerical implementation: To implement a new linear constitutive law in a finite-element code, one has to know the complete set of matrices needed to model the associated anisotropic behavior. In that regard, our result is a precious guideline, as it tells you how many matrices there are and how to construct them. This is illustrated in the case of three-dimensional strain gradient elasticity in [Auffray et al. 2013].

Constitutive tensors symmetry classes. In mechanics, constitutive laws are usually expressed in terms of tensorial relations between the gradients of primary variables and their fluxes [Gu and He 2011]. As is well known, this feature is not restricted to linear behaviors, since tensorial relations appear in the tangential formulation of nonlinear ones [Triantafyllidis and Bardenhagen 1996]. It is also known that a general tensorial relation can be divided into classes according to its symmetry properties. Such classes are known in mechanics as symmetry classes [Forte and Vianello 1996], and in mathematical physics as isotropic classes or strata [Abud and Sartori 1983; Auffray et al. 2011].

In the case of second-order tensors, the determination of symmetry classes is rather simple. Using spectral analysis it can be concluded that any second-order symmetric tensor ${ }^{1}$ can either be orthotropic $\left(\left[\mathrm{D}_{2}\right]\right)$, transverse isotropic $([\mathrm{O}(2)])$, or isotropic ([SO(3)]). Such tensors are commonly used to describe, for example, heat conduction and electric permittivity.

For higher-order tensors, the determination of the set of symmetry classes is more involved, and is mostly based on an approach introduced in [Forte and Vianello 1996] for the case of elasticity. Let us briefly detail this case.

The vector space of elasticity tensors, denoted by $\mathbb{E}$ la throughout this paper, is the subspace of fourth-order tensors endowed with the following index symmetries:

Minor symmetries: $E_{i j k l}=E_{j i k l}=E_{j i l k}$.

Major symmetry: $E_{i j k l}=E_{k l i j}$.

Symmetries will be specified using notation such as $E_{(i j)(k l)}$, where (..) indicates invariance under permutation of the indices in parentheses, and $\ldots \ldots$ indicates invariance with respect to permutations of the underlined blocks. Index symmetries encode the physics described by the mathematical operator. The minor symmetries

\footnotetext{
${ }^{1}$ Such a tensor is related to a symmetric matrix, which can be diagonalized in an orthogonal basis. The stated result is related to this diagonalization.
} 
stem from the fact that rigid body motions do not induce deformation (the symmetry of $\varepsilon$ ), and that the material is not subjected to volumic couple (the symmetry of $\sigma$ ). The major symmetry is the consequence of the existence of free energy. An elasticity tensor, $\mathbf{E}$, can be viewed as a symmetric linear operator on $\mathbb{T}_{(i j)}$, the space of symmetric second-order tensors. According to [Forte and Vianello 1996], for the classical action of $\mathrm{SO}(3)$, Ela is divided into eight symmetry classes (see page 183 for the notation):

$$
[\mathbb{E l a}]=\left\{[\mathbb{1}],\left[\mathrm{Z}_{2}\right],\left[\mathrm{D}_{2}\right],\left[\mathrm{D}_{3}\right],\left[\mathrm{D}_{4}\right],[\mathrm{O}(2)],[\mathrm{O}],[\mathrm{SO}(3)]\right\},
$$

which correspond, respectively, to the following physical classes: ${ }^{2}$ triclinic, monoclinic, orthotropic, trigonal, tetragonal, transverse isotropic, cubic, and isotropic. Besides this fundamental result, the interest of the Forte and Vianello paper was to provide a general method to determine the symmetry classes of any tensor space [Auffray et al. 2011]. Other results have been obtained by this method since then:

\begin{tabular}{|clcrl|}
\hline Property & Tensor & $\begin{array}{c}\text { Number } \\
\text { of classes }\end{array}$ & Action & Studied in \\
Photoelasticity & $T_{(i j)(k l)}$ & 12 & $\mathrm{SO}(3)$ & [Forte and Vianello 1997] \\
Piezoelectricity & $T_{(i j) k}$ & 15 & $\mathrm{O}(3)$ & [Geymonat and Weller 2002] \\
$\begin{array}{c}\text { Flexoelectricity } \\
\begin{array}{c}\text { A set of tensors } \\
\text { of order six }\end{array}\end{array} T_{(i j) k l}$ & 12 & $\mathrm{SO}(3)$ & [Le Quang and He 2011] \\
\hline
\end{tabular}

The limitations of the Forte-Vianello approach. The method introduced by Forte and Vianello is actually the most general. ${ }^{3}$ But, at the same time, it suffers from at least two limitations:

(1) The computation of the harmonic decomposition.

(2) The specificity of the study for each kind of tensor.

In its original setting, the method requires the computation of the explicit harmonic decomposition of the studied tensor, that is, its decomposition into the sum of its $\mathrm{SO}(3)$-irreducible components, also known as harmonic tensors ${ }^{4}$. Its explicit computation, which is generally based on an algorithm introduced by Spencer [1970], turns out to be intractable in practice as the tensorial order increases. But

\footnotetext{
${ }^{2}$ These symmetry classes are subgroups of the group $\mathrm{SO}(3)$ of space rotations. This is because the elasticity tensor is of even order. To treat odd-order tensors, the full orthogonal group $\mathrm{O}(3)$ has to be considered.

${ }^{3}$ Some other methods can be found in the literature, such as counting the symmetry planes [Chadwick et al. 2001], or studying the SU(2)-action on Ela [Bóna et al. 2004], and others, but these methods are difficult to generalize to arbitrary vector spaces.

${ }^{4}$ Harmonic tensors are completely symmetric and traceless. They inherit this name because of a well-known isomorphism in $\mathbb{R}^{3}$ between these tensors and harmonic polynomials [Backus 1970].
} 
this is not a real problem, since the only information needed is the number of different harmonic tensors of each order appearing in the decomposition, that is, the isotypic decomposition. Based on arguments presented in [Jerphagnon et al. 1978], there exists a direct procedure to obtain this isotypic decomposition from the tensor index symmetries [Auffray 2008]. Such an approach has been used in [Le Quang et al. 2012] to obtain the symmetry classes of sixth-order tensors.

As each kind of tensor space requires specific study, this specificity constitutes the other limitation of the method. This remark has to be considered together with the observation that, for even-order tensors it seems that there exist only two possibilities. Namely, a tensor space has as many classes as

- the full symmetric tensor space (for example, Ela is divided into eight classes, like the full symmetric tensor space [Forte and Vianello 1996]), or

- the generic tensor space ${ }^{5}$ (other fourth-order tensor spaces such as those of photoelasticity [Forte and Vianello 1997], flexoelectricity [Le Quang and $\mathrm{He}$ 2011], etc., are divided into 12 classes, like the generic tensor space).

The same observation can also be made for second and sixth-order tensors [Le Quang et al. 2012]. Understanding the general rule behind this observation would be an important result in mechanics. Its practical implication is the direct determination of the number and the type of symmetry classes for any constitutive law, no matter its order. This result is valuable for understanding generalized continuum theories, in which higher-order tensors are involved in constitutive laws.

Organization of the paper. In Section 2, the main results of this paper, Theorems I, II, and III, are stated. As an application, the symmetry classes of the even-order constitutive tensor spaces of Mindlin second strain-gradient elasticity are determined. Results concerning the sixth-order coupling tensor and the eighth-order second strain-gradient tensor are given for the first time. Obtaining the same results with the Forte-Vianello approach would have been much more difficult. Other sections are dedicated to the construction of our proofs. In Section 3, the mathematical framework used to obtain our result is introduced. Thereafter, we study the symmetry classes of a couple of harmonic tensors, which is the main purpose of the tool named the clips operator. We then give the associated results for couples of SO(3)-closed subgroups (Theorem 4.6 and Table 2). Thanks to these results, and with the help of previous work on the topic [Ihrig and Golubitsky 1984], we obtain in Section 5 some general results concerning symmetry classes for general evenorder tensors. In Section 6 our main results are finally proved. The Appendix is devoted to proofs and the calculus of clips operations.

${ }^{5}$ The $n$-th order generic tensor is a $n$-th order tensor with no index symmetry. 


\section{Main results}

In this section, our main results are stated. In the first subsection, the construction of constitutive tensor spaces (CTS) is discussed. This construction allows us to formulate our main results in the next subsection. Finally, the application of these results to Mindlin second strain-gradient elasticity (SSGE) is considered. Precise mathematical definitions of the symmetry classes are given in Section 3.

Construction of CTS. Linear constitutive laws are linear maps between the gradients of primary physical quantities and their fluxes. Each of these physical quantities (see Table 1 on the next page) is in fact related to subspaces ${ }^{6}$ of tensors spaces; these subspaces will be called state tensor spaces (STS). These STS will be the primitive notion from which the CTS will be constructed.

Notation. $\mathscr{L}(F, G)$ will indicate the vector space of linear maps from $F$ to $G$.

Now we consider two STS, $\mathbb{E}_{1}=\mathbb{T}_{G}$ and $\mathbb{E}_{2}=\mathbb{T}_{f}$, respectively of order $p$ and order $q$, possibly with index symmetries. As a consequence, they belong to subspaces of $\bigotimes^{p} \mathbb{R}^{3}$ and $\bigotimes^{q} \mathbb{R}^{3}$. A constitutive tensor $C$ is a linear map between $\mathbb{E}_{1}$ and $\mathbb{E}_{2}$, that is, an element of the space $\mathscr{L}\left(\mathbb{E}_{1}, \mathbb{E}_{2}\right)$. This space is isomorphic, modulo the use of an euclidean metric, to $\mathbb{E}_{1} \otimes \mathbb{E}_{2}$. Physical properties lead to some index symmetries on $C \in \mathbb{E}_{1} \otimes \mathbb{E}_{2}$; thus the vector space of such $C$ is some vector subspace $\mathbb{T}_{C}$ of $\mathbb{E}_{1} \otimes \mathbb{E}_{2}$.

Now, each of the spaces $\mathbb{E}_{1}, \mathbb{E}_{2}$, and $\mathbb{E}_{1} \otimes \mathbb{E}_{2}$ has natural $\mathrm{O}(3)$ actions. In this paper, we are concerned with cases in which $p+q=2 n$. In such a situation, it is known that the $\mathrm{O}(3)$-action on $\mathbb{E}_{1} \otimes \mathbb{E}_{2}$ reduces to that of $\mathrm{SO}(3)$ [Forte and Vianello 1996]. We therefore have

$$
\mathscr{L}\left(\mathbb{E}_{1}, \mathbb{E}_{2}\right) \simeq \mathbb{E}_{1} \otimes \mathbb{E}_{2} \subset \mathbb{T}^{p} \otimes \mathbb{T}^{q}=\mathbb{T}^{p+q=2 n} .
$$

Here are some examples of this construction:

\begin{tabular}{|c|c|c|c|c|}
\hline Property & $\mathbb{E}_{1}$ & $\mathbb{E}_{2}$ & $\begin{array}{l}\text { Tensor product } \\
\text { for CTS }\end{array}$ & $\begin{array}{l}\text { Number } \\
\text { of classes }\end{array}$ \\
\hline Elasticity & $\mathbb{T}_{(i j)}$ & $\mathbb{T}_{(i j)}$ & Symmetric & 8 \\
\hline Photoelasticity & $\mathbb{T}_{(i j)}$ & $\mathbb{T}_{(i j)}$ & Standard & 12 \\
\hline Flexoelectricity & $\mathbb{T}_{(i j) k}$ & $\mathbb{T}_{i}$ & Standard & 12 \\
\hline First-gradient elasticity & $\mathbb{T}_{(i j) k}$ & $\mathbb{T}_{(i j) k}$ & Symmetric & 17 \\
\hline
\end{tabular}

This table shows two kinds of CTS, describing respectively:

- coupled physics (tensors such as photoelasticity and flexoelectricity, encoding the coupling between two different physics), and

\footnotetext{
${ }^{6}$ Because of some symmetries.
} 


\begin{tabular}{|lll|}
\hline Physical notion & Mathematical object & Mathematical space \\
Gradient & Tensor state $\mathbf{T}_{1} \in \bigotimes^{p} \mathbb{R}^{3}$ & $\mathbb{\mathbb { T }}_{G}$ : tensor space with \\
& index symmetries \\
Fluxes of gradient & Tensor state $\mathbf{T}_{2} \in \bigotimes^{q} \mathbb{R}^{3}$ & $\mathbb{T}_{f}:$ tensor space with \\
& & index symmetries \\
Linear constitutive law & $C \in \mathscr{L}\left(\mathbb{T}_{G}, \mathbb{T}_{f}\right)$ & $\mathbb{T}_{C} \subset \mathscr{L}\left(\mathbb{T}_{G}, \mathbb{T}_{f}\right)$ \\
\hline
\end{tabular}

Table 1. Physical and mathematical links.

- proper physics (tensors such as classical and first-gradient elasticities, describing a single physical phenomenon).

On the mathematical side this implies:

- Coupled physics: the spaces $\mathbb{E}_{1}$ and $\mathbb{E}_{2}$ may differ, and when $\mathbb{E}_{1}=\mathbb{E}_{2}$ linear maps are not self-adjoint.

- Proper physics: we have $\mathbb{E}_{1}=\mathbb{E}_{2}$ and linear maps are self-adjoint. ${ }^{7}$

Therefore, the elasticity tensor is a self-adjoint linear map between the vector space of deformation tensors and the vector space of stress tensors. These two spaces are modeled on $\mathbb{T}_{(i j)}$. The vector space of elasticity tensors is therefore completely determined by $\mathbb{T}_{(i j)}$ and the symmetric nature of the tensor product, that is, $\mathbb{E l a}=\mathbb{T}_{(i j)} \otimes{ }^{S} \mathbb{T}_{(k l)}$, where $\otimes^{S}$ denotes the symmetric tensor product. On the side of coupling tensors, flexoelectricity is a linear map between $\mathbb{E}_{1}=\mathbb{T}_{(i j) k}$, the space of deformation gradients, and $\mathbb{E}_{2}=\mathbb{\mathbb { V }}_{l}$, the electric polarization; therefore $\mathbb{F l e x}=\mathbb{T}_{(i j) k} \otimes \mathbb{T}_{l}$.

Symmetry classes of even-order tensor spaces. Consider an even-order CTS $\mathbb{T}^{2 n}$. It is known [Jerphagnon et al. 1978] that this space can be decomposed orthogonally ${ }^{8}$ into a full symmetric space and a complementary one which is isomorphic to a tensor space of order $2 n-1$, that is:

$$
\mathbb{T}^{2 n}=\mathbb{S}^{2 n} \oplus \mathbb{C}^{2 n-1} \text {. }
$$

Let us introduce:

$\mathbb{S}^{2 n}:$ the vector space of $2 n$-th order completely symmetric tensors.

$\mathbb{G}^{2 n}$ : the vector space of $2 n$-th order tensors with no index symmetries. ${ }^{9}$

The following observation is obvious:

$$
\mathbb{S}^{2 n} \subseteq \mathbb{T}^{2 n} \subseteq \mathbb{G}^{2 n},
$$

\footnotetext{
${ }^{7}$ This is a consequence of the assumption of the existence of a free energy.

${ }^{8}$ The related dot product is constructed by $2 n$ products of the $\mathbb{R}^{3}$ canonical one.

${ }^{9}$ Formally this space is constructed as $\mathbb{G}^{2 n}=\bigotimes^{2 n} \mathbb{R}^{3}$.
} 
and therefore, if we denote by $\mathfrak{I}$ the operator which gives to a tensor space the set of its symmetry classes, we obtain:

$$
\Im\left(\mathbb{S}^{2 n}\right) \subseteq \Im\left(\mathbb{T}^{2 n}\right) \subseteq \mathfrak{I}\left(\mathbb{G}^{2 n}\right) .
$$

The symmetry group of even-order tensors is conjugate to $\mathrm{SO}(3)$-closed subgroups [Zheng and Boehler 1994; Forte and Vianello 1996]. Classification of SO(3)-closed subgroups is a classical result that can be found in many references [Ihrig and Golubitsky 1984; Sternberg 1994]. These subgroups are, up to conjugacy:

Lemma 2.1. Every closed subgroup of $\mathrm{SO}(3)$ is conjugate to precisely one group from the following list:

$$
\left\{\mathbb{1}, \mathrm{Z}_{n}, \mathrm{D}_{n}, \mathscr{T}, \mathcal{O}, \mathscr{T}, \mathrm{SO}(2), \mathrm{O}(2), \mathrm{SO}(3)\right\} .
$$

Among these groups, we can distinguish:

Planar groups: $\left\{\mathbb{1}, \mathrm{Z}_{n}, \mathrm{D}_{n}, \mathrm{SO}(2), \mathrm{O}(2)\right\}$, which are $\mathrm{O}(2)$-closed subgroups.

Exceptional groups: $\{\mathscr{T}, \mathcal{O}, \mathscr{I}, \mathrm{SO}(3)\}$, of which the first three are rotational symmetry groups of Platonic polyhedra.

Let us detail first the set of planar subgroups. We fix a base $(\mathbf{i} ; \mathbf{j} ; \mathbf{k})$ of $\mathbb{R}^{3}$, and denote by $\mathbf{Q}(\mathbf{v} ; \theta) \in \mathrm{SO}(3)$ the rotation about $\mathbf{v} \in \mathbb{R}^{3}$, with angle $\theta \in[0 ; 2 \pi)$.

- $\mathbb{1}$ is the identity.

- $\mathrm{Z}_{n}(n \geq 2)$ is the cyclic group of order $n$, generated by the $n$-fold rotation $\mathbf{Q}(\mathbf{k} ; \theta=2 \pi / n)$. which is the symmetry group of a chiral polygon.

- $\mathrm{D}_{n}(n \geq 2)$ is the dihedral group of order $2 n$ generated by $\mathrm{Z}_{n}$ and $\mathbf{Q}(\mathbf{i} ; \pi)$, which is the symmetry group of a regular polygon.

- $\mathrm{SO}(2)$ is the subgroup of rotations $\mathbf{Q}(\mathbf{k} ; \theta)$ with $\theta \in[0 ; 2 \pi)$.

- $\mathrm{O}(2)$ is the subgroup generated by $\mathrm{SO}(2)$ and $\mathbf{Q}(\mathbf{i} ; \pi)$.

The classes of exceptional subgroups are: $\mathscr{T}$, the tetrahedral group of order 12 which fixes a tetrahedron, $\mathbb{O}$, the octahedral group of order 24 which fixes an octahedron (or a cube), and $\mathscr{I}$, the subgroup of order 60 which fixes an icosahedron (or a dodecahedron).

In Section 6, the symmetry classes of $\mathbb{S}^{2 n}$ and $\mathbb{G}^{2 n}$ are obtained:

Lemma 2.2. The symmetry classes of $\mathbb{S}^{2 n}$ are:

$$
\begin{aligned}
\mathfrak{I}\left(\mathbb{S}^{2}\right) & =\left\{\left[\mathrm{D}_{2}\right],[\mathrm{O}(2)],[\mathrm{SO}(3)]\right\}, \\
\mathfrak{I}\left(\mathbb{S}^{4}\right)=\left\{[\mathbb{1}],\left[\mathrm{Z}_{2}\right],\left[\mathrm{D}_{2}\right],\left[\mathrm{D}_{3}\right],\left[\mathrm{D}_{4}\right],[\mathrm{O}(2)],[\mathrm{O}],[\mathrm{SO}(3)]\right\}, & \\
\mathfrak{I}\left(\mathbb{S}^{2 n}\right)=\left\{[\mathbb{1}],\left[\mathrm{Z}_{2}\right], \ldots,\left[\mathrm{Z}_{2(n-1)}\right],\left[\mathrm{D}_{2}\right], \ldots,\left[\mathrm{D}_{2 n}\right],[\mathrm{O}(2)],[\mathcal{T}],[\mathrm{O}],[\mathcal{F}],[\mathrm{SO}(3)]\right\}, & \text { if } n \geq 3 .
\end{aligned}
$$


Lemma 2.3. The symmetry classes of $\mathbb{G}^{2 n}$ are:

$$
\begin{aligned}
\mathfrak{I}\left(\mathbb{G}^{2}\right) & =\left\{[\mathbb{1}],\left[\mathrm{Z}_{2}\right],\left[\mathrm{D}_{2}\right],[\mathrm{SO}(2)],[\mathrm{O}(2)],[\mathrm{SO}(3)]\right\}, \\
\mathfrak{I}\left(\mathbb{G}^{4}\right) & =\left\{[\mathbb{1}],\left[\mathrm{Z}_{2}\right],\left[\mathrm{Z}_{3}\right],\left[\mathrm{Z}_{4}\right],\left[\mathrm{D}_{2}\right],\left[\mathrm{D}_{3}\right],\left[\mathrm{D}_{4}\right],[\mathrm{SO}(2)],[\mathrm{O}(2)],[\mathscr{T}],[\mathcal{O}],[\mathrm{SO}(3)]\right\}, \\
\mathfrak{I}\left(\mathbb{G}^{2 n}\right) & =\left\{[\mathbb{1}],\left[\mathrm{Z}_{2}\right], \ldots,\left[\mathrm{Z}_{2 n}\right],\left[\mathrm{D}_{2}\right], \ldots,\left[\mathrm{D}_{2 n}\right],[\mathrm{SO}(2)],[\mathrm{O}(2)],[\mathscr{T}],[\mathcal{O}],[\mathscr{F}],[\mathrm{SO}(3)]\right\}, \\
& \text { if } n \geq 3 .
\end{aligned}
$$

The following table lists how many classes there are for each $n$ :

\begin{tabular}{|rrrc|}
\hline$n=$ & 1 & 2 & $\geq 3$ \\
$\# \Im\left(\mathbb{S}^{2 n}\right)$ & 3 & 8 & $2(2 n+1)$ \\
$\# \Im\left(\mathbb{G}^{2 n}\right)$ & 6 & 12 & $4 n+5$ \\
\hline
\end{tabular}

The symmetry classes of $\mathbb{T}^{2 n}$ are clarified by the following theorem:

Theorem I. Let $\mathbb{T}^{2 n}$ be a tensor space. Then either $\mathfrak{I}\left(\mathbb{T}^{2 n}\right)=\mathfrak{I}\left(\mathbb{S}^{2 n}\right)$ or $\mathfrak{I}\left(\mathbb{T}^{2 n}\right)=$ $\Im\left(\mathbb{G}^{2 n}\right)$.

In other words, the number and type of classes are the same as those of

- $\mathbb{S}^{2 n}$, the space of $2 n$-order completely symmetric tensors, in which case the number of classes is minimal, or

- $\mathbb{G}^{2 n}$, the space of $2 n$-order generic tensors, in which case the number of classes is maximal.

In fact, as specified by the following theorems, in most situations the number of classes is indeed maximal.

Theorem II (coupling tensors). Let us consider $\mathbb{T}^{2 p}$ the space of coupling tensors between two physics described by two tensor vector spaces $\mathbb{E}_{1}$ and $\mathbb{E}_{2}$. If these tensor spaces are of orders greater than or equal to one, then $\Im\left(\mathbb{T}^{2 p}\right)=\Im\left(\mathbb{G}^{2 p}\right)$.

Theorem III (proper tensors). Let us consider $\mathbb{T}^{2 p}$, the space of tensors of a proper physics described by the tensor vector space $\mathbb{E}$. If this tensor space is of order $p \geq 3$, and is solely defined in terms of its index symmetries, then $\mathfrak{I}\left(\mathbb{T}^{2 p}\right)=\mathfrak{I}\left(\mathbb{G}^{2 p}\right)$.

Remark 2.4. Exceptions occur for

$p=1$, when the space of symmetric second-order tensors is obtained, and $p=2$, when, in the case of $\mathbb{T}_{(i j)}$, the space of elasticity tensors is obtained.

In each of these situations the number of classes is minimal. There is no other situation where this case occurs. It should therefore be concluded that the space of elasticity tensors is exceptional. 
Second strain-gradient elasticity (SSGE). Application of the former theorems will be made on the even-order tensors of SSGE. First, the constitutive equations will be summed up, and then the results will be stated. It worth noting that obtaining the same results with the Forte-Vianello approach would have been far more complicated.

Constitutive laws. In the second strain-gradient theory of linear elasticity [Mindlin 1965; Forest et al. 2011], the constitutive law gives the symmetric Cauchy stress tensor ${ }^{10} \sigma^{(2)}$ and the hyperstress tensors $\tau^{(3)}$ and $\omega^{(4)}$ in terms of the infinitesimal strain tensor $\varepsilon^{(2)}$ and its gradients $\eta^{(3)}=\varepsilon^{(2)} \otimes \nabla$ and $\kappa^{(4)}=\varepsilon^{(2)} \otimes \nabla \otimes \nabla$ through the three linear relations:

$$
\begin{aligned}
\sigma^{(2)} & =\mathbf{E}^{(4)}: \varepsilon^{(2)}+\mathbf{M}^{(5)} \therefore \eta^{(3)}+\mathbf{N}^{(6)}:: \kappa^{(4)}, \\
\tau^{(3)} & =\mathbf{M}^{T(5)}: \varepsilon+\mathbf{A}^{(6)} \therefore \eta^{(3)}+\mathbf{O}^{(7)}:: \kappa^{(4)}, \\
\omega^{(4)} & =\mathbf{N}^{T(6)}: \varepsilon^{(2)}+\mathbf{O}^{T(7)} \therefore \eta^{(3)}+\mathbf{B}^{(8)}:: \kappa^{(4)},
\end{aligned}
$$

where $:, \therefore$, and :: denote, respectively, the double, third, and fourth contracted products. Above, ${ }^{11} \sigma_{(i j)}, \varepsilon_{(i j)}, \tau_{(i j) k}, \eta_{(i j) k}=\varepsilon_{(i j), k}, \omega_{(i j)(k l)}$, and $\kappa_{(i j)(k l)}=\varepsilon_{(i j),(k l)}$ are, respectively, the matrix components of $\sigma^{(2)}, \varepsilon^{(2)}, \tau^{(3)}, \eta^{(3)}, \omega^{(4)}$, and $\kappa^{(4)}$ relative to an orthonormal basis $(\mathbf{i} ; \mathbf{j} ; \mathbf{k})$ of $\mathbb{R}^{3}$. And $E_{(i j)} \frac{(l m)}{}, M_{(i j)(l m) n}, N_{(i j)(k l)(m n)}$, $A_{\underline{(i j) k} \underline{(l m) n},}, O_{(i j) k(l m)(n o)}$, and $B_{(i j)(k l)} \underline{(m n)(o p)}$ are the matrix components of the related elastic stiffness tensors.

Symmetry classes. The symmetry classes of the elasticity tensors and of the first strain-gradient elasticity tensors has been studied in [Forte and Vianello 1996; Le Quang et al. 2012]. Hence, here we solely consider the spaces of coupling tensors $\mathbf{N}^{(6)}$ and of second strain-gradient elasticity tensors $\mathbf{B}^{(8)}$.

- We define $\mathbb{C}$ es to be the space of coupling tensors between classical elasticity and second strain-gradient elasticity:

$$
\mathbb{C} e s=\left\{\mathbf{N}^{(6)} \in \mathbb{G}^{6} \mid N_{(i j)(k l)(m n)}\right\} .
$$

A direct application of Theorem II leads to the result that $\Im(\mathbb{C} e s)=\left\{[\mathbb{1}],\left[\mathrm{Z}_{2}\right], \ldots,\left[\mathrm{Z}_{6}\right],\left[\mathrm{D}_{2}\right], \ldots,\left[\mathrm{D}_{6}\right],[\mathrm{SO}(2)],[\mathrm{O}(2)],[\mathscr{T}],[\mathrm{O}],[\mathscr{g}],[\mathrm{SO}(3)]\right\}$

Therefore $\mathbb{C}$ es is divided into 17 symmetry classes.

- We define Sgr to be the space of second strain-gradient elasticity tensors:

$$
\operatorname{Sgr}=\left\{\mathbf{O}^{(8)} \in \mathbb{G}^{8} \mid O_{\underline{(i j)(k l)}} \underline{(m n)(o p)}\right\} .
$$

${ }^{10}$ In this subsection only, tensor orders will be indicated by superscripts in parentheses.

${ }^{11}$ The comma classically indicates the partial derivative with respect to spatial coordinates. Superscript $T$ denotes transposition. Transposition is defined by permuting the $p$ first indices with the $q$ last, where $p$ is the tensorial order of the image of a $q$-order tensor. 
A direct application of Theorem III leads to the result that $\mathfrak{I}(\operatorname{Sgr})=\left\{[\mathbb{1}],\left[\mathrm{Z}_{2}\right], \ldots,\left[\mathrm{Z}_{8}\right],\left[\mathrm{D}_{2}\right], \ldots,\left[\mathrm{D}_{8}\right],[\mathrm{SO}(2)],[\mathrm{O}(2)],[\mathscr{T}],[\mathrm{O}],[\mathscr{F}],[\mathrm{SO}(3)]\right\}$

Therefore Sgr is divided into 21 symmetry classes.

\section{Mathematical framework}

In this section the mathematical framework of symmetry analysis is introduced. In the first two subsections the notions of symmetry group and class are introduced; the last is devoted to the introduction of irreducible spaces. The presentation is rather general, and will be specialized to tensor spaces only at the end of the section.

Isotropy/symmetry groups. Let $\rho$ be a representation of a compact real Lie group ${ }^{12}$ $G$ on a finite dimensional $\mathbb{R}$-linear space $\mathscr{E}$ :

$$
\rho: G \rightarrow \mathrm{GL}(\mathscr{E}) .
$$

This action will also be denoted by

$$
g \cdot \mathbf{x}=\rho(g)(\mathbf{x}),
$$

where $g \in G$ and $\mathbf{x} \in \mathscr{E}$. For any element of $\mathscr{E}$, the set of operations $g$ in $G$ leaving this element invariant is defined as

$$
\Sigma_{\mathbf{x}}:=\{g \in G \mid g \cdot \mathbf{x}=\mathbf{x}\} .
$$

This set is known to physicists as the symmetry group of $\mathbf{x}$ and to mathematicians as the stabilizer or isotropy subgroup of $\mathbf{x}$. Owing to $G$-compactness, every isotropy subgroup is a closed subgroup of $G$. Conversely, a dual notion can be defined for $G$-elements. For any subgroup $K$ of $G$, the set of $K$-invariant elements in $\mathscr{E}$ is defined as

$$
\mathscr{E}^{K}:=\{\mathbf{x} \in \mathscr{E} \mid k \cdot \mathbf{x}=\mathbf{x} \text { for all } k \in K\}
$$

Such a set is referred to as a fixed point set and is a linear subspace of $\mathscr{E}$. In this context we will set $\mathrm{d}(K)=\operatorname{dim} \mathscr{E}^{K}$. It has to be observed that fixed-point sets are group inclusion reversing, that is, for subgroups $K_{1}$ and $K_{2}$ of $G$, we have the property

$$
K_{1} \subset K_{2} \Longrightarrow \mathscr{E}^{K_{2}} \subset \mathscr{E}^{K_{1}}
$$

For a given isotropy group $K$, the former sets are linked by the property

$$
\mathbf{x} \in \mathscr{E}^{\mathscr{E}} K \Longrightarrow K \subset \Sigma_{\mathbf{x}}
$$

${ }^{12}$ In the following $G$ will always represent a compact real Lie group, so this specification will mostly be omitted. 
Isotropy/symmetry classes. We aim to describe objects that have the same symmetry properties but may differ by their orientations in space. The first point is to define the set of all the positions an object can have. To that aim we consider the $G$-orbit of an element $\mathbf{x}$ of $\mathscr{E}$ :

$$
\operatorname{Orb}(\mathbf{x}):=\{g \cdot \mathbf{x} \mid g \in G\} \subset \mathscr{E} .
$$

Due to $G$-compactness this set is a submanifold of $\mathscr{E}$. Elements of $\operatorname{Orb}(\mathbf{x})$ will be said to be $G$-related. A fundamental observation is that $G$-related vectors have conjugate symmetry groups. More precisely, ${ }^{13}$

$$
\operatorname{Orb}(\mathbf{x})=\operatorname{Orb}(\mathbf{y}) \Longrightarrow \Sigma_{\mathbf{x}}=g \Sigma_{\mathbf{y}} g^{-1} \text { for some } g \in G \text {. }
$$

Let us define the conjugacy class of a subgroup $K \subset G$ by

$$
[K]=\left\{K^{\prime} \subset G \mid K^{\prime}=g K g^{-1} \text { for some } g \in G\right\} .
$$

An isotropy class (or symmetry class) $[\Sigma]$ is defined as the conjugacy class of an isotropy subgroup $\Sigma$. This definition implies that there exists a vector $\mathbf{x} \in \mathscr{E}$ such that $\Sigma=\Sigma_{\mathbf{X}}$ and $\Sigma^{\prime} \in[\Sigma]$; furthermore $\Sigma^{\prime}=g \Sigma g^{-1}$ for some $g \in G$. The notion of isotropy class is a good notion to define the symmetry property of an object modulo its orientation: a symmetric group is related to a specific vector, but we deal with orbits, which are related to isotropy classes because of (3-1). Due to $G$ compactness there is only a finite number of isotropy classes [Bredon 1972], and we introduce the notation

$$
\Im(\mathscr{I}):=\left\{[\mathbb{1}] ;\left[\Sigma_{1}\right] ; \ldots ;\left[\Sigma_{l}\right]\right\},
$$

the set of all isotropy classes. In the case $G=\mathrm{SO}(3)$ this result is known as Hermann's theorem [Herman 1945; Auffray 2008]. The elements of $\mathfrak{I}(\mathscr{E})$ are conjugate to $\mathrm{SO}$ (3)-closed subgroups; this collection was introduced in Lemma 2.1.

Irreducible spaces. For every linear subspace $\mathscr{F}$ of $\mathscr{E}$, we set

$$
g \cdot \mathscr{F}:=\{g \cdot \mathbf{x} \mid g \in G, \mathbf{x} \in \mathscr{F}\}
$$

and we say that $\mathscr{F}$ is $G$-stable if $g \cdot \mathscr{F} \subset \mathscr{F}$ for every $g \in G$. It is clear that, for every representation, the subspaces $\{0\}$ and $\mathscr{E}$ are always $G$-stable. If, for a representation $\rho$ on $\mathscr{E}$, the only $G$-invariant spaces are the proper ones, the representation will be said to be irreducible. For a compact Lie group, the Peter-Weyl theorem [Sternberg 1994] ensures that every representation can be split into a direct sum of irreducible

\footnotetext{
${ }^{13}$ With the classical coset notation, if $H$ is a subgroup of $G$ and $g \in G$ is not in the subgroup $H$, then a left coset of $H$ in $G$ is defined

$$
g H=\{g h: h \in H\},
$$
}

and symmetrically for a right coset. 
ones. Furthermore, in the case $G=\mathrm{SO}(3)$, those irreducible representations are explicitly known.

There is a natural action of $\mathrm{SO}(3)$ on the space of $\mathbb{R}^{3}$-harmonic polynomials. If $p$ is a harmonic polynomial and $\mathbf{x} \in \mathbb{R}^{3}$, then for every $g \in \mathrm{SO}$ (3) we write

$$
g \cdot p(\mathbf{x})=p\left(g^{-1} \cdot \mathbf{x}\right) .
$$

Harmonic polynomials form a graded vector space, and to each subspace of a given degree is associated an $\mathrm{SO}(3)$-irreducible representation. $\mathscr{H}^{k}$ will be the vector space of harmonic polynomials of degree $k$, with $\operatorname{dim} \mathscr{H}^{k}=2 k+1$. If we take a vector space $V$ to be an $\mathrm{SO}(3)$-representation, it can be decomposed into $\mathrm{SO}(3)$-irreducible spaces:

$$
V=\bigoplus \mathscr{H}^{k_{i}}
$$

Grouping together irreducible spaces of the same order, one obtains the $\mathrm{SO}(3)$ isotypic decomposition of a representation:

$$
V=\bigoplus_{i=0}^{n} \alpha_{i} \mathscr{H}^{i},
$$

where $\alpha_{i}$ is the multiplicity of the irreducible space $\mathscr{H}^{i}$ in the decomposition, and $n$ is the order of the highest-order irreducible space of the decomposition.

Application to tensor spaces. In mechanics, $V$ is a vector subspace of $\bigotimes^{p} \mathbb{R}^{3}$. In $\mathbb{R}^{3}$ there exists an isomorphism, $\phi$, between harmonic polynomial spaces and harmonic tensor spaces [Backus 1970; Forte and Vianello 1996]. Therefore all that has been said for harmonic polynomials can be translated in terms of harmonic tensors. A detailed discussion on this isomorphism can be found in [Backus 1970]. Therefore $\mathbb{H}^{k}=\varphi\left(\mathscr{H}^{k}\right)$ is the space of harmonic tensors, that is, the space of completely symmetric and traceless tensors. According to this isomorphism, any tensor space $\mathbb{T}^{n}$ can be decomposed into $\mathrm{SO}(3)$-irreducible tensors:

$$
\mathbb{T}^{n}=\bigoplus_{i=0}^{n} \alpha_{i} \boxplus^{i} .
$$

The symmetry group of $\mathbf{T} \in \mathbb{T}^{n}$ is the intersection of the symmetry groups of all its harmonic components: ${ }^{14}$

$$
\Sigma_{\mathbf{T}}=\bigcap_{i=0}^{n}\left(\bigcap_{j=0}^{\alpha_{i}} \Sigma_{\mathrm{H}^{i, j}}\right) .
$$

${ }^{14}$ In the notation $\mathrm{H}^{i, j}$, the first superscript refers to the order of the harmonic tensor, while the second indexes the multiplicity of $\mathrm{H}^{i}$ in the decomposition. 
In the same way, $\Im\left(\mathbb{T}^{n}\right)$ will be obtained as a function of the symmetry classes of the irreducible representations involved in the harmonic decomposition of $\mathbb{T}^{n}$. The symmetry classes of $\mathrm{SO}(3)$-irreducible representations are explicitly known [Ihrig and Golubitsky 1984; Golubitsky et al. 1988]; what is unknown is how to combine these results to determine the symmetry classes of $V$ (or $\mathbb{T}^{n}$ ).

\section{The clips operation}

The aim of this section is to construct symmetry classes of a reducible representation from irreducible ones. With that goal a new class-operator, named the clips operator, will be defined. The main result of this section is given in Table 2, which contains all clips operations between $\mathrm{SO}(3)$-closed subgroups. It is worth noting that this table contains more results than strictly needed for the proofs of our theorems. Nevertheless, we believe that these results are interesting on their own and may find application in other problems. The explicit proofs of these results can be found in the Appendix.

Here we consider the intersection of two symmetry classes only. Extensions to more general reducible representations will be treated in Section 5. Let us start with the following lemma:

Lemma 4.1. Let $\mathscr{E}$ be a representation of a compact Lie group $G$ that splits into a direct sum of two $G$-stable subspaces:

$$
\mathscr{E}=\mathscr{E}_{1} \oplus \mathscr{E}_{2} \text {, where } g \cdot \mathscr{E}_{1} \subset \mathscr{E}_{1} \text { and } g \cdot \mathscr{E}_{2} \subset \mathscr{E}_{2} \text { for all } g \in G \text {. }
$$

If we denote by $\mathfrak{I}$ the set of all isotropy classes associated with $\mathscr{E}$ and by $\mathfrak{I}_{i}$ the set of all isotropy classes associated with $\mathscr{E}_{i}(i=1,2)$, then $[\Sigma] \in \mathfrak{I}$ if and only if there exist $\left[\Sigma_{1}\right] \in \mathfrak{I}_{1}$ and $\left[\Sigma_{2}\right] \in \mathfrak{I}_{2}$ such that $\Sigma=\Sigma_{1} \cap \Sigma_{2}$.

Proof. If we take $\left[\Sigma_{1}\right] \in \mathfrak{I}_{1}$ and $\left[\Sigma_{2}\right] \in \mathfrak{I}_{2}$, we know there exist two vectors $\mathbf{x}_{1} \in \mathscr{E}_{1}$ and $\mathbf{x}_{2} \in \mathscr{E}_{2}$ such that $\Sigma_{i}=\Sigma_{\mathbf{x}_{i}}(i=1,2)$. Then, let $\mathbf{x}:=\mathbf{x}_{1}+\mathbf{x}_{2}$.

For every $g \in \Sigma_{1} \cap \Sigma_{2}$ we have $g \cdot \mathbf{x}_{1}+g \cdot \mathbf{x}_{2}=\mathbf{x}_{1}+\mathbf{x}_{2}=\mathbf{x}$; thus $\Sigma_{1} \cap \Sigma_{2} \subset \Sigma_{\mathbf{x}}$. Conversely for every $g \in \Sigma_{\mathbf{x}}$ we have

$$
g \cdot \mathbf{x}=\mathbf{x}=g \cdot \mathbf{x}_{1}+g \cdot \mathbf{x}_{2} .
$$

But, since the $\mathscr{E}_{i}$ are $G$-stable and form a direct sum, we conclude that $g \cdot \mathbf{x}_{i}=\mathbf{x}_{i}$ $(i=1,2)$. The reverse inclusion is proved.

The other implication is similar: if we take $[\Sigma] \in \mathfrak{I}$ then we have $\Sigma=\Sigma_{\mathbf{x}}$ for some $\mathbf{x} \in \mathscr{E}$, and $\mathbf{x}$ can be decomposed into $\mathbf{x}_{1}+\mathbf{x}_{2}$. The same proof as above shows that $\Sigma=\Sigma_{\mathbf{x}_{1}} \cap \Sigma_{\mathbf{x}_{2}}$.

Lemma 4.1 shows that the isotropy classes of a direct sum are related to intersections of isotropy subgroups. But as intersection of classes is meaningless, the 
results cannot be directly extended. To solve this problem, a tool called the clips operator will be introduced. We will make sure of a lemma:

Lemma 4.2. For every two $G$-classes $\left[\Sigma_{i}\right](i=1,2)$, and for every $g_{1}$ and $g_{2}$ in $G$, there exists $g=g_{1}^{-1} g_{2}$ in $G$ such that

$$
\left[g_{1} \Sigma_{1} g_{1}^{-1} \cap g_{2} \Sigma_{2} g_{2}^{-1}\right]=\left[\Sigma_{1} \cap g \Sigma_{2} g^{-1}\right] .
$$

Proof. Let $g=g_{1}^{-1} g_{2}$ and

$$
\Sigma=g_{1} \Sigma_{1} g_{1}^{-1} \cap g_{2} \Sigma_{2} g_{2}^{-1} .
$$

For every $\gamma \in \Sigma$ we have $\gamma=g_{1} \gamma_{1} g_{1}^{-1}=g_{2} \gamma_{2} g_{2}^{-1}$ for some $\gamma_{i} \in \Sigma_{i}(i=1,2)$; then

$$
g_{1} \gamma g_{1}^{-1}=\gamma_{1} \in \Sigma_{1} \text { and } g_{1} \gamma g_{1}^{-1}=g \gamma_{2} g^{-1} \in g \Sigma_{2} g^{-1} \text {. }
$$

Thus we have $g_{1} \Sigma g_{1}^{-1} \subset \Sigma_{1} \cap g \Sigma_{2} g^{-1}$, and conversely. Since $g_{1} \Sigma g_{1}^{-1}$ is conjugate to $\Sigma$, we have proved the lemma.

Definition 4.3 (clips operator). We define the action of the clips operator $\odot$ on $G$-classes $\left[\Sigma_{1}\right]$ and $\left[\Sigma_{2}\right]$ by setting

$$
\left[\Sigma_{1}\right] \odot\left[\Sigma_{2}\right]:=\left\{\left[\Sigma_{1} \cap g \Sigma_{2} g^{-1}\right] \mid g \in G\right\},
$$

which is a subset of $G$-classes.

If we denote by $\mathbb{1}$ the identity subgroup, we have some immediate properties:

Proposition 4.4. For every $G$-class $[\Sigma]$ we have

$$
[\mathbb{1}] \odot[\Sigma]=\{[\mathbb{1}]\} \text { and }[G] \odot[\Sigma]=\{[\Sigma]\} \text {. }
$$

Given two $G$-representations $\mathscr{E}_{1}$ and $\mathscr{E}_{2}$, if we denote by $\mathfrak{I}_{i}$ the set of all isotropy classes of $\mathscr{E}_{i}$, the action of the clips operator can be extended to these sets via

$$
\mathfrak{I}_{1} \odot \mathfrak{I}_{2}:=\bigcup_{\substack{\Sigma_{1} \in \mathfrak{I}_{1} \\ \Sigma_{2} \in \mathfrak{I}_{2}}}\left[\Sigma_{1}\right] \odot\left[\Sigma_{2}\right] .
$$

Then, by Lemma 4.1, we obtain:

Corollary 4.5. For every two G-representations $\mathscr{E}_{1}$ and $\mathscr{E}_{2}$, if $\mathfrak{I}_{1}$ denotes the isotropy classes of $\mathscr{E}_{1}$ and $\mathfrak{I}_{2}$ the isotropy classes of $\mathscr{E}_{2}$, then $\mathfrak{I}_{1} \odot \mathfrak{I}_{2}$ are all the isotropy classes of $\mathscr{E}_{1} \oplus \mathscr{E}_{2}$.

Theorem 4.6. For any two $\mathrm{SO}(3)$-closed subgroups $\Sigma_{1}$ and $\Sigma_{2}$, we have $\mathbb{1} \in$ $\left[\Sigma_{1}\right] \odot\left[\Sigma_{2}\right]$. The remaining classes in the clips product $\left[\Sigma_{1}\right] \odot\left[\Sigma_{2}\right]$ are given in Table 2. 


\begin{tabular}{|c|c|c|c|c|c|c|c|}
\hline () & {$\left[\mathrm{Z}_{n}\right]$} & {$\left[\mathrm{D}_{n}\right]$} & {$[\mathscr{T}]$} & [0] & {$[\mathscr{I}]$} & {$[\mathrm{SO}(2)]$} & {$[\mathrm{O}(2)]$} \\
\hline$\left[\mathrm{Z}_{m}\right]$ & {$\left[\mathrm{Z}_{d}\right]$} & & & & & & \\
\hline$\left[\mathrm{D}_{m}\right]$ & $\begin{array}{l}{\left[\mathrm{Z}_{d_{2}}\right]} \\
{\left[\mathrm{Z}_{d}\right]}\end{array}$ & $\begin{array}{c}{\left[\mathrm{Z}_{d_{2}}\right]} \\
{\left[\mathrm{Z}_{d^{\prime}}\right],\left[\mathrm{Z}_{d z}\right]} \\
{\left[\mathrm{Z}_{d}\right],\left[\mathrm{D}_{d}\right]}\end{array}$ & & & & & \\
\hline$[\mathscr{T}]$ & $\begin{array}{l}{\left[\mathrm{Z}_{d_{2}}\right]} \\
{\left[\mathrm{Z}_{d_{3}}\right]}\end{array}$ & $\begin{array}{c}{\left[\mathrm{Z}_{2}\right]} \\
{\left[\mathrm{Z}_{d_{3}}\right],\left[\mathrm{D}_{d_{2}}\right]}\end{array}$ & $\begin{array}{l}{\left[\mathrm{Z}_{2}\right]} \\
{\left[\mathrm{Z}_{3}\right]} \\
{[\mathscr{T}]}\end{array}$ & & & & \\
\hline$[0]$ & $\begin{array}{l}{\left[\mathrm{Z}_{d_{2}}\right]} \\
{\left[\mathrm{Z}_{d_{3}}\right]} \\
{\left[\mathrm{Z}_{d_{4}}\right]}\end{array}$ & $\begin{array}{c}{\left[\mathrm{Z}_{2}\right]} \\
{\left[\mathrm{Z}_{d_{3}}\right],\left[\mathrm{Z}_{d_{4}}\right]} \\
{\left[\mathrm{D}_{d_{2}}\right],\left[\mathrm{D}_{d_{3}}\right]} \\
{\left[\mathrm{D}_{d_{4}}\right]}\end{array}$ & $\begin{array}{l}{\left[\mathrm{Z}_{2}\right]} \\
{\left[\mathrm{Z}_{3}\right]} \\
{[\mathscr{T}]}\end{array}$ & $\begin{array}{c}{\left[\mathrm{Z}_{2}\right]} \\
{\left[\mathrm{D}_{2}\right],\left[\mathrm{Z}_{3}\right]} \\
{\left[\mathrm{D}_{3}\right],\left[\mathrm{Z}_{4}\right]} \\
{\left[\mathrm{D}_{4}\right],[\mathrm{O}]}\end{array}$ & & & \\
\hline$[\mathscr{I}]$ & $\begin{array}{l}{\left[\mathrm{Z}_{d_{2}}\right]} \\
{\left[\mathrm{Z}_{d_{3}}\right]} \\
{\left[\mathrm{Z}_{d_{5}}\right]}\end{array}$ & $\begin{array}{c}{\left[\mathrm{Z}_{2}\right]} \\
{\left[\mathrm{Z}_{d_{3}}\right],\left[\mathrm{Z}_{d_{5}}\right]} \\
{\left[\mathrm{D}_{d_{2}}\right]} \\
{\left[\mathrm{D}_{d_{3}}\right],\left[\mathrm{D}_{d_{5}}\right]}\end{array}$ & $\begin{array}{l}{\left[\mathrm{Z}_{2}\right]} \\
{\left[\mathrm{Z}_{3}\right]} \\
{[\mathscr{T}]}\end{array}$ & $\begin{array}{c}{\left[\mathrm{Z}_{2}\right]} \\
{\left[\mathrm{Z}_{3}\right],\left[\mathrm{D}_{3}\right]} \\
{[\mathscr{T}]}\end{array}$ & $\begin{array}{c}{\left[\mathrm{Z}_{2}\right]} \\
{\left[\mathrm{Z}_{3}\right],\left[\mathrm{D}_{3}\right]} \\
{\left[\mathrm{Z}_{5}\right],\left[\mathrm{D}_{5}\right]} \\
{[\mathscr{\Phi}]}\end{array}$ & & \\
\hline$[\mathrm{SO}(2)]$ & {$\left[\mathrm{Z}_{n}\right]$} & $\begin{array}{l}{\left[\mathrm{Z}_{2}\right]} \\
{\left[\mathrm{Z}_{n}\right]}\end{array}$ & $\begin{array}{l}{\left[Z_{2}\right]} \\
{\left[Z_{3}\right]}\end{array}$ & $\begin{array}{c}{\left[\mathrm{Z}_{2}\right]} \\
{\left[\mathrm{Z}_{3}\right],\left[\mathrm{Z}_{4}\right]}\end{array}$ & $\begin{array}{c}{\left[\mathrm{Z}_{2}\right]} \\
{\left[\mathrm{Z}_{3}\right],\left[\mathrm{Z}_{5}\right]}\end{array}$ & {$[\mathrm{SO}(2)]$} & \\
\hline$[\mathrm{O}(2)]$ & $\begin{array}{c}{\left[\mathrm{Z}_{d_{2}}\right]} \\
{\left[\mathrm{Z}_{n}\right]}\end{array}$ & $\begin{array}{l}{\left[\mathrm{Z}_{2}\right]} \\
{\left[\mathrm{D}_{n}\right]}\end{array}$ & $\begin{array}{l}{\left[\mathrm{D}_{2}\right]} \\
{\left[\mathrm{Z}_{3}\right]}\end{array}$ & $\begin{array}{c}{\left[\mathrm{D}_{2}\right]} \\
{\left[\mathrm{D}_{3}\right],\left[\mathrm{D}_{4}\right]}\end{array}$ & $\begin{array}{c}{\left[\mathrm{D}_{2}\right]} \\
{\left[\mathrm{D}_{3}\right],\left[\mathrm{D}_{5}\right]}\end{array}$ & $\begin{array}{c}{\left[\mathrm{Z}_{2}\right]} \\
{[\mathrm{SO}(2)]}\end{array}$ & $\begin{array}{c}{\left[\mathrm{Z}_{2}\right]} \\
{[\mathrm{O}(2)]}\end{array}$ \\
\hline
\end{tabular}

Table 2. Action of the clips operation on $\mathrm{SO}(3)$-subgroups.

Conventions: $\mathrm{Z}_{1}:=\mathrm{D}_{1}:=\mathbb{1} ; d_{2}:=\operatorname{gcd}(n, 2) ; d_{3}:=\operatorname{gcd}(n, 3)$; $d_{5}:=\operatorname{gcd}(n, 5) ; d_{2}^{\prime}:=\operatorname{gcd}(m, 2) ; d z:=2$ if $d=1,1$ otherwise; $d_{4}:=4$ if $4 \mid n, 1$ otherwise.

\section{Isotropy classes of harmonic tensors}

We now turn to the construction of the symmetry classes of a reducible representation from its irreducible components. The first subsection states the main results on symmetry classes of irreducible representations. Thereafter we derive from the results of the previous section the basic properties of reducible representations. These results will be used in Section 6 to prove the theorems stated in Section 2.

From now on, all results will be expressed in terms of tensor spaces.

Isotropy classes of irreducibles. The following result was obtained in [Ihrig and Golubitsky 1984; Golubitsky et al. 1988]: 
Theorem 5.1. Let $\mathrm{SO}(3)$ act on $\mathbb{U}^{k}$. The following groups are symmetry classes of $\mathbb{\boxplus}^{k}$ :

(a) $\mathbb{1}$ for $k \geq 3$.

(b) $\mathrm{Z}_{n}(n \geq 2)$ for $n \leq k$ when $k$ is odd, $n \leq k / 2$ when $k$ is even.

(c) $\mathrm{D}_{n}(n \geq 2)$ for $n \leq k$.

(d) $\mathscr{T}$ for $k=3,6,7$ or $k \geq 9$.

(e) o for $k \neq 1,2,3,5,7,11$.

(f) $\mathscr{I}$ for $k=6,10,12,15,18$ or $k \geq 20$ and $k \neq 23,29$.

(g) $\mathrm{SO}(2)$ for $k$ odd.

(h) $\mathrm{O}(2)$ for $k$ even.

(i) $\mathrm{SO}(3)$ for any $k$.

For future purposes, let us introduce some notation. For each integer $k$, we let:

$$
\begin{aligned}
\Gamma_{\mathscr{T}(k)} & := \begin{cases}\mathscr{T} & \text { if } \mathscr{T} \in \mathfrak{I}^{k}, \\
\varnothing & \text { otherwise; }\end{cases} \\
\Gamma_{\mathscr{O}(k)} & := \begin{cases}\mathcal{O} & \text { if } \mathcal{O} \in \mathfrak{I}^{k}, \\
\varnothing & \text { otherwise; }\end{cases} \\
\Gamma_{\mathscr{I}(k)} & := \begin{cases}\mathscr{I} & \text { if } \mathscr{I} \in \mathfrak{I}^{k}, \\
\varnothing & \text { otherwise; }\end{cases} \\
\Sigma(k) & := \begin{cases}\mathrm{SO}(2) & \text { if } \mathrm{SO}(2) \in \mathfrak{I}^{k}, \\
\varnothing & \text { otherwise; }\end{cases} \\
\Omega(k) & := \begin{cases}\mathrm{O}(2) & \text { if } \mathrm{O}(2) \in \mathfrak{I}^{k}, \\
\varnothing & \text { otherwise; }\end{cases}
\end{aligned}
$$

where $\mathfrak{I}^{k}$ is the set of symmetry classes of $\mathbb{\sharp}^{k}$.

Isotropy classes of direct sum. We have this obvious lemma, directly deduced from Theorem 5.1:

Lemma 5.2.

$$
\begin{array}{ll}
\Gamma_{\mathscr{T}(k)} \neq \varnothing \Longrightarrow \quad\left\{\left[\mathrm{D}_{2}\right],\left[\mathrm{D}_{3}\right]\right\} \subset \mathfrak{I}^{k}, \\
\Gamma_{\mathscr{O}(k)} \neq \varnothing \Longrightarrow\left\{\left[\mathrm{D}_{2}\right],\left[\mathrm{D}_{3}\right],\left[\mathrm{D}_{4}\right]\right\} \subset \mathfrak{I}^{k}, \\
\Gamma_{\mathscr{I}(k)} \neq \varnothing \Longrightarrow\left\{\left[\mathrm{D}_{2}\right], \ldots,\left[\mathrm{D}_{5}\right]\right\} \subset \mathfrak{I}^{k} .
\end{array}
$$

We denote by $\mathfrak{I}(k, n)$ the $(n-1)$-fold self clips product of $\mathfrak{I}^{k}$, which is the set of isotropy classes of a $n$-tuple of $k$-th harmonic tensors [Auffray et al. 2011], that is, $n \mathbb{\natural}^{k}$. The basic operations are, for all integers $k \geq 1$ and $n \geq 2$,

$$
\mathfrak{I}(k, n):=\mathfrak{I}^{k} \odot \Im(k, n-1) \quad \text { and } \quad \mathfrak{I}(k, 1):=\mathfrak{I}^{k} \text {. }
$$


On the simple example of $\mathbb{H}^{2}$, the following fact can be observed:

$$
\mathfrak{I}(2, n):=\mathfrak{I}^{2} \odot \Im(2, n-1)=\mathfrak{I}^{2} \odot \mathfrak{I}^{2}=\left\{[\mathbb{1}],\left[\mathrm{Z}_{2}\right],\left[\mathrm{D}_{2}\right],[\mathrm{O}(2)],[\mathrm{SO}(3)]\right\} .
$$

This result can be generalized:

Corollary 5.3. For all integers $n \geq 2$ and $k \geq 2$, the isotropy classes of $n \mathbb{\boxplus}^{k}$ are

$$
\begin{aligned}
\mathfrak{I}(k, n)= & \mathfrak{I}^{k} \odot \mathfrak{I}^{k} \\
= & \left\{[\mathbb{1}],\left[\mathrm{Z}_{2}\right], \ldots,\left[\mathrm{Z}_{k}\right],\right. \\
& \left.\quad\left[\mathrm{D}_{2}\right], \ldots,\left[\mathrm{D}_{k}\right],\left[\Gamma_{\mathscr{T}(k)}\right],\left[\Gamma_{\mathscr{O}(k)}\right],\left[\Gamma_{\mathscr{I}(k)}\right],[\Sigma(k)],[\Omega(k)],[\operatorname{SO}(3)]\right\} .
\end{aligned}
$$

Proof. From Theorem 5.1 we know that $\left[\mathrm{D}_{l}\right] \in \mathfrak{I}^{k}$ for $2 \leq l \leq k$; furthermore, $[\mathrm{SO}(3)] \in \mathfrak{I}^{k}$. Then from Proposition 4.4 we know that, for all integers $2 \leq l \leq k$ we will have (by induction), for all $n \geq 2,\left[\mathrm{D}_{l}\right] \in \mathfrak{I}(k, n)$. Then, when we compute $\Im(k, n) \odot \mathfrak{I}^{k}$ we will have $\left[\mathrm{D}_{l}\right] \odot\left[\mathrm{D}_{l}\right]=\left\{[\mathbb{1}],\left[\mathrm{Z}_{l}\right],\left[\mathrm{D}_{l}\right]\right\}$. Neither $[\mathrm{O}(2)]$ nor $[\mathrm{SO}(2)]$, with cyclic or dihedral conjugacy classes, generates other cases. The same occurs for the clips product of cyclic groups. Now, because of Lemma 5.2 we also see that no exceptional conjugacy class generates other cases.

Corollary 5.4. For all integers $2 \leq 2 p<2 q$, we have

$$
\begin{aligned}
& \mathfrak{I}(2 p, 2 q):= \mathfrak{I}^{2 p} \odot \mathfrak{I}^{2 q} \\
&=\left\{[\mathbb{1}],\left[\mathrm{Z}_{2}\right], \ldots,\left[\mathrm{Z}_{\max (q ; 2 p)}\right],\left[\mathrm{D}_{2}\right], \ldots,\left[\mathrm{D}_{2 q}\right],\left[\Gamma_{T}(2 p) \cup \Gamma_{T}(2 q)\right],\right. \\
&\left.\quad\left[\Gamma_{O}(2 p) \cup \Gamma_{O}(2 q)\right],\left[\Gamma_{I}(2 p) \cup \Gamma_{I}(2 q)\right],[\mathrm{O}(2)],[\mathrm{SO}(3)]\right\} .
\end{aligned}
$$

Proof. Because $[\mathrm{SO}(3)] \in \mathfrak{I}^{k_{i}}(i=1,2)$, it is clear that we will have all $\left[\mathrm{D}_{l}\right]$ for $2 \leq l \leq 2 q$. Then we will have all $\left[\mathrm{Z}_{i}\right] \odot[\mathrm{SO}(3)]$, for $1 \leq i \leq p$. We also have $\left[\mathrm{Z}_{j}\right] \in\left[\mathrm{D}_{j}\right] \odot\left[\mathrm{D}_{j}\right]$, for $1 \leq j \leq 2 q$; this shows that

$$
\left\{[\mathbb{1}],\left[\mathrm{Z}_{2}\right], \ldots,\left[\mathrm{Z}_{\max (q ; 2 p)}\right]\right\} \subset \Im \mathfrak{I}[2 p, 2 q] .
$$

Now, we can observe that the clips product of dihedral groups and $[\mathrm{O}(2)]$ does not generate cyclic groups, and Lemma 5.2 shows that no other cases can be generated with exceptional subgroups.

\section{Isotropy classes of constitutive tensors}

The symmetry classes of an even-order tensor space. Let us consider the CTS $\mathbb{T}^{2 n}$. It is known that this space can be decomposed orthogonally into a full symmetric space and a complementary one which is isomorphic to a tensor space of order $2 n-1$ [Jerphagnon et al. 1978]:

$$
\mathbb{T}^{2 n}=\mathbb{S}^{2 n} \oplus \mathbb{C}^{2 n-1}
$$


Let us consider the $\mathrm{SO}(3)$-isotypic decomposition of $\mathbb{T}^{2 n}$ :

$$
\mathbb{T}^{2 n}=\bigoplus_{k=0}^{2 n} \alpha_{k} \mathbb{H}^{k}, \text { with } \alpha_{2 n}=1
$$

The part related to $\mathbb{S}^{2 n}$ solely contains even-order harmonic tensors with multiplicity one [Jerphagnon et al. 1978], that is,

$$
\mathbb{S}^{2 n}=\bigoplus_{k=0}^{n} \mathbb{M}^{2 k} \text { and } \mathbb{C}^{2 n-1}=\bigoplus_{k=0}^{2 n-1} \alpha_{k}^{\prime} \mathbb{M}^{k} \text { with } \alpha_{k}^{\prime}=\left\{\begin{array}{l}
\alpha_{k} \text { for } k \text { odd } \\
\alpha_{k}-1 \text { for } k \text { even }
\end{array}\right.
$$

Using the clips operator, the symmetry classes of $\mathbb{T}^{2 n}$ can be expressed:

$$
\mathfrak{I}\left(\mathbb{T}^{2 n}\right):=\mathfrak{I}\left(\mathbb{S}^{2 n}\right) \odot \mathfrak{I}\left(\mathbb{C}^{2 n-1}\right) .
$$

Let us first determine the symmetry classes of $\mathbb{S}^{2 n}$. Using the results of the previous section, we have:

\section{Lemma 6.1.}

$$
\begin{aligned}
\Im\left(\mathbb{S}^{2}\right) & =\left\{\left[\mathrm{D}_{2}\right],[\mathrm{O}(2)],[\mathrm{SO}(3)]\right\} \\
\mathfrak{I}\left(\mathbb{S}^{4}\right) & =\left\{[\mathbb{1}],\left[\mathrm{Z}_{2}\right],\left[\mathrm{D}_{2}\right],\left[\mathrm{D}_{3}\right],\left[\mathrm{D}_{4}\right],[\mathrm{O}(2)],[\mathrm{O}],[\mathrm{SO}(3)]\right\} \\
\mathfrak{I}\left(\mathbb{S}^{2 n}\right) & =\left\{[\mathbb{1}],\left[\mathrm{Z}_{2}\right], \ldots,\left[\mathrm{Z}_{2(n-1)}\right],\left[\mathrm{D}_{2}\right], \ldots,\left[\mathrm{D}_{2 n}\right],[\mathrm{O}(2)],[\mathscr{T}],[\mathrm{O}],[\mathcal{F}],[\mathrm{SO}(3)]\right\} \\
& \text { if } n \geq 3 .
\end{aligned}
$$

In particular,

$$
\# \Im\left(\mathbb{S}^{2}\right)=3, \quad \# \Im\left(\mathbb{S}^{4}\right)=8, \quad \# \Im\left(\mathbb{S}^{2 n}\right)=2(2 n+1) .
$$

Proof. The case $n=1$ is obtained as a direct application of Theorem 5.1 and Proposition 4.4. For $n \geq 2$, let us consider Corollary 5.4 in the case of $k_{1}=2(n-1)$ and $k_{2}=2 n$ :

$$
\begin{aligned}
& \Im(2(n-1), 2 n) \\
& \quad:=\left\{[\mathbb{1}],\left[\mathrm{Z}_{2}\right], \ldots,\left[\mathrm{Z}_{2(n-1)}\right],\left[\mathrm{D}_{2}\right], \ldots,\left[\mathrm{D}_{2 n}\right],\left[\Gamma_{T}(2(n-1)) \cup \Gamma_{T}(2 n)\right],\right. \\
& \left.\quad\left[\Gamma_{O}(2(n-1)) \cup \Gamma_{O}(2 n)\right],\left[\Gamma_{I}(2(n-1)) \cup \Gamma_{I}(2 n)\right],[\mathrm{O}(2)],[\mathrm{SO}(3)]\right\} .
\end{aligned}
$$

In the collection of planar isotropy classes, $\left[\mathrm{Z}_{2 n-1}\right]$ and $\left[\mathrm{Z}_{2 n}\right]$ are missing. It should be observed that the clips product $\mathfrak{I}[2(n-1), 2 n] \odot \mathfrak{I}^{2(n-2)}$ can never complete the sequence.

For exceptional groups it can be observed that for any $n \geq 3$ the $\mathrm{SO}(3)$-irreducible decomposition will contain $\mathbb{W}^{6}$. As $\{[\mathscr{T}],[\mathcal{O}],[\mathscr{G}]\}$ are isotropy classes for $\mathbb{T}^{6}$, it would be the same for any space that contains $\mathbb{W}^{6}$.

Therefore, for $n \geq 3$, 
$\Im\left(\mathbb{S}^{2 n}\right)=\left\{[\mathbb{1}],\left[\mathrm{Z}_{2}\right], \ldots,\left[\mathrm{Z}_{2(n-1)}\right],\left[\mathrm{D}_{2}\right], \ldots,\left[\mathrm{D}_{2 n}\right],[\mathrm{O}(2)],[\mathscr{T}],[\mathrm{O}],[\mathscr{F}],[\mathrm{SO}(3)]\right\}$ and \#I $\left(\mathbb{S}^{2 n}\right)=2(2 n+1)$.

For the case $n=2$, we obtain the same result but without the classes $\mathscr{T}$ and $\mathscr{I}$ and, in such a case, $\# \Im\left(\mathbb{S}^{4}\right)=8$.

Definition 6.2. For a given $\mathrm{SO}(3)$ representation on the tensor space $\mathbb{T}^{2 n}(n \geq 3)$, we define

$\mathscr{b}(2 n)$

$=\left\{[\mathbb{1}],\left[\mathrm{Z}_{2}\right], \ldots,\left[\mathrm{Z}_{2 n}\right],\left[\mathrm{D}_{2}\right], \ldots,\left[\mathrm{D}_{2 n}\right],[\mathrm{SO}(2)],[\mathrm{O}(2)],[\mathcal{T}],[\mathrm{O}],[\mathscr{G}],[\mathrm{SO}(3)]\right\}$.

We also define

$$
\begin{aligned}
\mathscr{C}(2) & =\left\{[\mathbb{1}],\left[\mathrm{Z}_{2}\right],\left[\mathrm{D}_{2}\right],[\mathrm{SO}(2)],[\mathrm{O}(2)],[\mathrm{SO}(3)]\right\} \\
\mathscr{C}(4) & =\left\{[\mathbb{1}],\left[\mathrm{Z}_{2}\right], \ldots,\left[\mathrm{Z}_{4}\right],\left[\mathrm{D}_{2}\right], \ldots,\left[\mathrm{D}_{4}\right],[\mathrm{SO}(2)],[\mathrm{O}(2)],[\mathscr{T}],[\mathrm{O}],[\mathrm{SO}(3)]\right\}
\end{aligned}
$$

One can observe that these sets are in fact all the isotropy classes allowed by Hermann's theorem, and we clearly have

$$
\# \mathscr{C}(2)=6, \quad \# \mathscr{C}(4)=12, \quad \# \mathscr{C}(2 n, n \geq 3)=4 n+5 .
$$

Definition 6.3. Let $\mathbb{T}^{2 n}$ be a tensor space which $\mathrm{SO}(3)$-irreducible decomposition is $\mathbb{T}^{2 n} \simeq \bigoplus_{k=0}^{2 n} \alpha_{k} \mathbb{T}^{k}$. $\mathbb{T}^{2 n}$ is said to be even-harmonic (EH) if $\alpha_{2 p+1}=0$ for each $0 \leq p \leq(n-1)$.

Lemma 6.4. The vector space $\mathbb{G}^{2 n}$ of $2 n$-th order tensors with no index symmetries is not $\mathrm{EH}$.

Proof. For $n \geq 1$, the induced reducible $\mathrm{SO}(3)$-representation on $\mathbb{G}^{2 n}=\bigotimes^{2 n} \mathbb{R}^{3}$ is constructed by tensorial products of the vectorial one. Such a construction implies odd-order tensors in the harmonic decomposition of $\mathbb{G}^{2 n}$.

Now we can prove Theorem I, which we restate in the following form:

Theorem I. Let $\mathbb{T}^{2 n}$ be a tensor space, with $n \geq 3$. If $\mathbb{T}^{2 n}$ is EH then $\Im\left(\mathbb{T}^{2 n}\right)=$ $\Im\left(\mathbb{S}^{2 n}\right)$; otherwise, $\mathfrak{I}\left(\mathbb{T}^{2 n}\right)=\mathfrak{I}\left(\mathbb{G}^{2 n}\right)$.

Proof. We consider the $\mathrm{SO}(3)$-irreducible decomposition of $\mathbb{T}^{2 n}$, which can be written $\mathbb{T}^{2 n} \simeq \mathbb{S}^{2 n} \oplus \mathbb{C}^{2 n-1}$. The following inclusions always hold:

$$
\Im\left(\mathbb{S}^{2 n}\right) \subseteq \Im\left(\mathbb{T}^{2 n}\right) \subseteq \Im\left(\mathbb{G}^{2 n}\right) \subseteq \mathscr{C}(2 n) .
$$

If $\mathbb{T}^{2 n}$ is not $\mathrm{EH}$, there exists at least one $k \in \mathbb{N}$ such that $\alpha_{2 k+1} \neq 0$; then

$$
\mathfrak{I}\left(\mathbb{S}^{2 n}\right) \odot[\mathrm{SO}(2)] \subseteq \Im\left(\mathbb{S}^{2 n}\right) \odot \mathfrak{I}\left(\mathbb{C}^{2 n-1}\right)=\mathfrak{I}\left(\mathbb{T}^{2 n}\right),
$$

since, from Theorem 5.1, any odd-order harmonic tensor admits [SO(2)] as a symmetry class. From Lemma 6.1, dihedral groups are contained up to $2 n$ in $\mathfrak{I}\left(\mathbb{S}^{2 n}\right)$, 
so the missing cyclic groups of $\mathfrak{I}\left(\mathbb{S}^{2 n}\right)$ are obtained by clips products with [SO(2)]. Therefore

$$
\Im\left(\mathbb{S}^{2 n}\right) \odot \mathrm{SO}(2)=\mathscr{C}(2 n) ;
$$

hence $\mathfrak{I}\left(\mathbb{T}^{2 n}\right)=\mathscr{C}(2 n)$. Since $\mathbb{G}^{2 n}$ is not EH, $\mathfrak{I}\left(\mathbb{G}^{2 n}\right)=\mathscr{C}(2 n)$. We conclude, as desired, that if $\mathbb{T}^{2 n}$ is not EH then $\mathfrak{I}\left(\mathbb{T}^{2 n}\right)=\mathfrak{I}\left(\mathbb{G}^{2 n}\right)$.

Conversely, if $\mathbb{T}^{2 n}$ is $\mathrm{EH}, \mathbb{C}^{2 n-1}$ contains only even-order irreducible spaces and its leading harmonic spaces are, at most, of order $2(n-1)$. If the orders of the leading harmonic spaces are strictly less than $2(n-1)$, the same analysis as for Lemma 6.1 leads to the same conclusion. Now suppose that $\alpha_{2(n-1)}^{\prime} \geq 1$; using all the previous results, we have

$$
\begin{aligned}
\mathfrak{I}\left(\mathbb{T}^{2 n}\right) & =\mathfrak{I}\left(\mathbb{S}^{2 n}\right) \odot \Im\left(\mathbb{C}^{2 n-1}\right) \\
& =\left(\mathfrak{I}^{2 n} \odot \mathfrak{I}^{2(n-1)}\right) \odot\left(\mathfrak{I}\left(2(n-1), \alpha_{2(n-1)}^{\prime}\right) \odot \Im\left(2(n-2), \alpha_{2(n-2)}^{\prime}\right)\right) \\
& =\left(\mathfrak{I}^{2 n} \odot \mathfrak{I}(2(n-1), 2)\right)=\mathfrak{I}\left(\mathbb{S}^{2 n}\right) \odot \mathfrak{I}^{2(n-1)} .
\end{aligned}
$$

Since $\mathfrak{I}^{2(n-1)}$ does not contain [SO(2)], the missing classes cannot be generated; therefore $\mathfrak{I}\left(\mathbb{T}^{2 n}\right)=\mathfrak{I}\left(\mathbb{S}^{2 n}\right)$.

We must conclude, then, that for any $2 n$-order tensor space, the symmetry classes are the same as those of either $\mathbb{S}^{2 n}$ or $\mathbb{G}^{2 n}$. In the next subsection we investigate under what conditions each of the possibilities holds.

Construction of a CTS. This last subsection will be devoted to the proof of our main result. The space of constitutive tensors is a subspace of linear maps from $\mathbb{E}_{1}$ to $\mathbb{E}_{2}$. As seen in Section 2,

$$
\mathscr{L}\left(\mathbb{E}_{1}, \mathbb{E}_{2}\right) \simeq \mathbb{E}_{1} \otimes \mathbb{E}_{2} \subset \mathbb{T}^{p} \otimes \mathbb{T}^{q} \simeq \mathbb{T}^{2 n=p+q} .
$$

These vector spaces describe the physical quantities involved in the problem under study. We know, from the previous section, that any CTS has as many symmetry classes as either the complete symmetric tensor space, or the generic tensor space. Here we are interested in obtaining the conditions both on $\mathbb{E}_{1}$ to $\mathbb{E}_{2}$ and on the tensor product (symmetric or not) under which $\mathbb{T}^{2 n}$ is even-harmonic, and therefore has a minimal number of symmetry classes. Distinction will be made between coupling and proper tensor spaces, in the sense previously defined in Section 2.

Coupling tensor spaces. We consider here two STS given by their $\mathrm{SO}(3)$ isotypic decompositions:

$$
\mathbb{E}_{1}=\mathbb{T}^{p}=\bigoplus_{i=0}^{p} \beta_{i} \mathbb{M}^{i} \quad \text { and } \quad \mathbb{E}_{2}=\mathbb{T}^{q}=\bigoplus_{i=0}^{q} \gamma_{j} \mathbb{M}^{j},
$$

with $\beta_{p}=\gamma_{q}=1$.

Lemma 6.5. If $\mathbb{E}_{1} \neq \mathbb{E}_{2}, p>q$ and if $\mathbb{T}^{p} \otimes \mathbb{\mathbb { T }}^{q}$ is $E H$, then $\mathbb{T}^{p}$ is $E H$ and $\mathbb{T}^{q}=\mathbb{T}^{0}$. 
Proof. It is sufficient to consider the tensor product of the two leading irreducible spaces, and to use the Clebsch-Gordan product for SO(3) [Jerphagnon et al. 1978; Auffray 2008]. We obtain

$$
\mathbb{M}^{p} \otimes \mathbb{M}^{q}=\bigoplus_{i=|p-q|}^{p+q} \mathbb{\boxplus}^{i}
$$

Therefore $p$ must be even, and $q=0$. Therefore $\mathbb{T}^{q}=\gamma_{0} \mathbb{\boxplus}^{0}$, and by hypothesis $\gamma_{0}=1$. Thus $\mathbb{T}^{p}$ has to be $\mathrm{EH}$.

Lemma 6.6. If $\mathbb{E}_{1}=\mathbb{E}_{2}$ (and then $p=q$ ) and if $\mathbb{T}^{p} \otimes \mathbb{T}^{p}$ is $E H$ then $\beta_{i}=\gamma_{i}$. Furthermore, if $\mathscr{L}\left(\mathbb{E}_{1}\right)$ is not self-adjoint then $\mathbb{T}^{p}=\mathbb{U}^{0}$.

Proof. The demonstration is the same as the preceding proof.

As a direct application of the two preceding two lemmas, we have:

Theorem II. Consider $\mathbb{T}^{2 p}$, the space of coupling tensors between two physics described by two tensor vector spaces $\mathbb{E}_{1}$ and $\mathbb{E}_{2}$. If these tensor spaces have order at least one, then $\mathfrak{I}\left(\mathbb{T}^{2 n}\right)=\mathfrak{I}\left(\mathbb{G}^{2 n}\right)$.

Proper tensor spaces. In this case we have:

Lemma 6.7. Let $\mathbb{E}=\mathbb{E}_{1}=\mathbb{E}_{2}=\mathbb{T}^{p}$. Assume $\mathbb{T}^{2 p} \subset \mathscr{L}(\mathbb{E})$ is self-adjoint and $E H$. Then:

- If $p=2 m+1$, then $\mathbb{\square}^{2 m+1}=\mathbb{U}^{2 m+1}$.

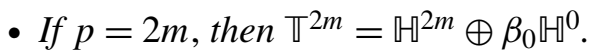

Proof. Because $\mathscr{L}(\mathbb{E})$ is self-adjoint the tensor product is replaced by the symmetric tensor product, and if $\mathbb{T}^{p}=\bigoplus_{i=0}^{p} \beta_{i} \mathbb{M}^{i}$, the symmetric tensor product $\mathbb{T}^{p} \otimes^{S} \mathbb{T}^{p}$ can be decomposed into a direct sum of

$$
\beta_{i}^{2} \mathbb{M}^{i} \otimes \mathbb{S}^{S} \mathbb{\boxplus}^{i} \text { and } \beta_{i} \beta_{j} \mathbb{M}^{i} \otimes \mathbb{M}^{j} \text {, with } i<j \in\{0, \ldots, p\},
$$

with the following Clebsch-Gordan rule for the symmetric product:

$$
\mathbb{M}^{k} \otimes \mathbb{M}^{k}=\bigoplus_{i=0}^{k} \mathbb{H}^{2 i} .
$$

Therefore, we cannot have the tensor product $\mathbb{M}^{i} \otimes \mathbb{W}^{j}$ for $1 \leq i \leq p-1$ and $i \neq j$; thus we deduce that

$$
\mathbb{T}^{p}=\beta_{0} \mathbb{\boxplus}^{0} \oplus \mathbb{\boxplus}^{p} \text { and } \mathbb{T}^{p} \otimes^{S} \mathbb{T}^{p}=\beta_{0} \mathbb{\boxplus}^{0} \oplus\left(\mathbb{\boxplus}^{p} \otimes^{S} \mathbb{\boxplus}^{p}\right) \oplus 2 \beta_{0} \mathbb{\boxplus}^{p} .
$$

Then either $p$ is odd, and $\beta_{0}=0$, or $p$ is even, and $\mathbb{T}^{p}=\beta_{0} \mathbb{H}^{0} \oplus \mathbb{M}^{p}$.

We therefore obtain: 
Theorem III. Consider $\mathbb{T}^{2 p}$, the space of tensors of a proper physics described by the tensor vector space $\mathbb{E}$. If this tensor space is of order $p \geq 3$, and is solely defined in terms of its index symmetries, then $\mathfrak{I}\left(\mathbb{T}^{2 n}\right)=\mathfrak{I}\left(\mathbb{G}^{2 n}\right)$.

Proof. Any tensor subspace defined in terms of its index symmetries contains, as a subspace, the space of full symmetric tensors. Since $p=3$, the harmonic decomposition of $\mathbb{S}^{3}$ does not satisfy the condition of Lemma 6.7. Direct application of this lemma leads to the conclusion.

Remark 6.8. It can be observed that CTS having a minimum number of classes can nevertheless be constructed. They consist in spaces of self-adjoint linear applications between harmonic spaces, which are defined both from complete symmetry under index permutations and a traceless property.

\section{Conclusion}

In this paper the symmetry class determination of even-order tensors has been studied. Based on a new geometric approach, a complete and general answer to this recurrent problem in continuum mechanics has been given. Application of our results solves problems directly that would have been difficult to manage with the Forte-Vianello method. As an example, and for the first time, the symmetry classes of the even-order tensors involved in Mindlin second strain-gradient elasticity were given. To reach this goal a geometric tool, called the clips operator, has been introduced. Its main properties and all the products for $\mathrm{SO}(3)$-closed-subgroups were also provided. We believe that these results may find applications in other contexts. Using the geometrical framework introduced in this paper, some extensions of the current method can be considered:

- Extending this approach to odd-order tensors.

- Taking into account the coexistence of different symmetry properties for the physical properties of architectured multimaterials.

These extensions will be the objects of forthcoming papers.

\section{Appendix: Clips operation on SO(3)-subgroups}

Here we establish results concerning the clips operator on $\mathrm{SO}(3)$-subgroups. The geometric idea to study the intersection of symmetry classes relies on the symmetry determination of composite figures the symmetry groups of which are the intersection of two elementary figures. As an example we consider the rotation $\mathbf{r}=\mathbf{Q}(\mathbf{k} ; \pi / 3)$; determining $\mathrm{D}_{4} \cap \mathbf{r} \mathrm{D}_{4} \mathbf{r}^{t}$ is tantamount to establishing the set of transformations letting the composite Figure 1 invariant. 


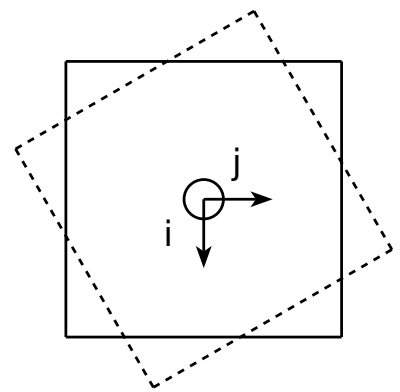

Figure 1. Composite figure associated with $\mathrm{D}_{4} \cap \mathbf{r D}_{4} \mathbf{r}^{t}$, where $\mathbf{r}=\mathbf{Q}(\mathbf{k} ; \pi / 3)$.

Parametrization of subgroups. We will define geometric elements for each $\mathrm{SO}(3)$ closed subgroup:

- The cyclic group $\mathrm{Z}_{n}$ is characterized by the $O z$ axis; it will be denoted by $\mathrm{Z}_{n}^{0}:=\mathrm{Z}_{n}$.

- The same convention is retained for the dihedral group $\mathrm{D}_{n}$, that is, $\mathrm{D}_{n}^{0}:=\mathrm{D}_{n}$.

- For the cube $\mathscr{C}_{0}$ (see Figure $2 \mathrm{a}$ ) we defined its vertex collection $\left\{A_{i}\right\}_{i=1 \ldots 8}=$ $( \pm 1 ; \pm 1 \pm 1) ; \mathscr{C}_{0}$ is $\mathbb{O}^{0}$-invariant.

- For the tetrahedron we consider Figure $2 \mathrm{a}$ and define $\mathscr{T}_{0}$ to be the tetrahedron $A_{1} A_{3} A_{7} A_{5} ; \mathscr{T}_{0}$ is $\mathscr{T}^{0}$-invariant.

- For the dodecahedron (see Figure $2 b$ ), we denote by $\mathscr{D}_{0}$ the figure with the following vertices (where $\phi$ is the golden ratio):

- twelve vertices of type $\left( \pm a / 2, \pm \phi^{2} a / 2,0\right)$ circularly permuted and

- eight vertices of a cube with coordinates $( \pm \phi a / 2, \pm \phi a / 2, \pm \phi a / 2)$.

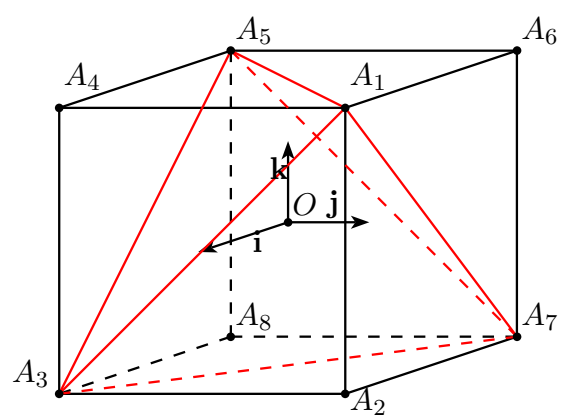

(a)

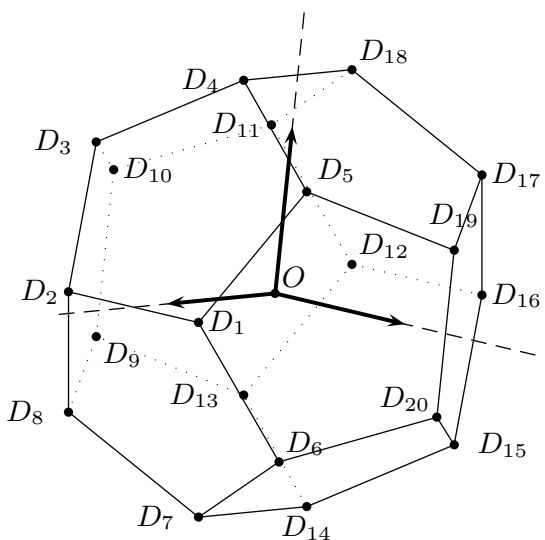

(b)

Figure 2. Cube $\mathscr{C}_{0}$ (a) and dodecahedron $\mathscr{D}_{0}(\mathrm{~b})$. 
Axes and subgroup classes. For every $\mathrm{SO}(3)$-subgroup, we defined its $g$ conjugate in the following way: $K^{g}=g K^{0} g^{t}$, where the superscript $g$ indicates the transformation, and 0 the initial configuration. To proceed towards our analysis we need to introduce the following group decomposition [Ihrig and Golubitsky 1984; Golubitsky et al. 1988].

Definition A.1. Let $K_{1}, K_{2}, \ldots, K_{s}$ be subgroups of $\Sigma$. Then $\Sigma$ is the direct union of the $K_{i}$ if

$$
\Sigma=\bigcup_{i=1}^{s} K_{i} \quad \text { and } \quad K_{i} \cap K_{j}=\{e\} \text { if } i \neq j .
$$

In this case we write $K=\biguplus_{i=1}^{s} K_{i}$.

We give some important details about the geometric structure of an $\mathrm{SO}(3)$ subgroup:

- $\mathrm{Z}_{n}^{0}$ is characterized by the $O z$ axis, generated by $\mathbf{k}$. For every rotation $g \in$ $\mathrm{SO}(3)$, we denote by $a$ the axis generated by $g \mathbf{k}$ and let $Z_{n}^{a}=Z_{n}^{g}$ indicate the rotation axis.

- $\mathrm{D}_{n}^{0}$ is characterized by its primary axis $O z$ and several secondary axes $b_{l}$. Therefore

$$
\mathrm{D}_{n}^{0}=\mathrm{Z}_{n}^{0} \biguplus_{l=0}^{n-1} \mathrm{Z}_{2}^{b_{l}}
$$

Each $b_{l}$ is perpendicular to $O z$. They are related by the $Z_{n}^{0}$ generator. $\mathrm{D}_{n}^{0}$ is chosen such that one $b_{l}$ is generated by $\mathbf{i}$. For every rotation $g \in \mathrm{SO}(3)$ we define $a$ - generated by $g \mathbf{k}$ - to be the primary axis and $b-$ generated by $g \mathbf{i}$ - to be the secondary one; this is denoted by

$$
\mathrm{D}_{n}^{a, b}=\mathrm{D}_{n}^{g}
$$

- The subgroup $\mathscr{T}^{0}$ can be split into a direct union of cyclic subgroups [Ihrig and Golubitsky 1984]:

$$
\mathscr{T}^{0}=\biguplus_{i=1}^{4} \mathrm{Z}_{3}^{v t_{i}} \uplus \biguplus_{j=1}^{3} \mathrm{Z}_{2}^{e t_{j}},
$$

where the vertex axes of the tetrahedron are written $v t_{i}$ and the edge axes $e t_{j}$; the details of these axes appear in Figure 2a. Each conjugate subgroup $\mathscr{T}^{g}$ will be characterized by the set of its axes $\left(g v t_{i}, g e t_{i}\right), g \in \mathrm{SO}(3)$.

- The octahedral subgroup $0^{0}$ splits into

$$
\mathrm{O}^{0}=\biguplus_{i=1}^{3} \mathrm{Z}_{4}^{f c_{i}} \uplus \biguplus_{j=1}^{4} \mathrm{Z}_{3}^{v c_{j}} \uplus \biguplus_{l=1}^{6} \mathrm{Z}_{2}^{e c_{l}},
$$




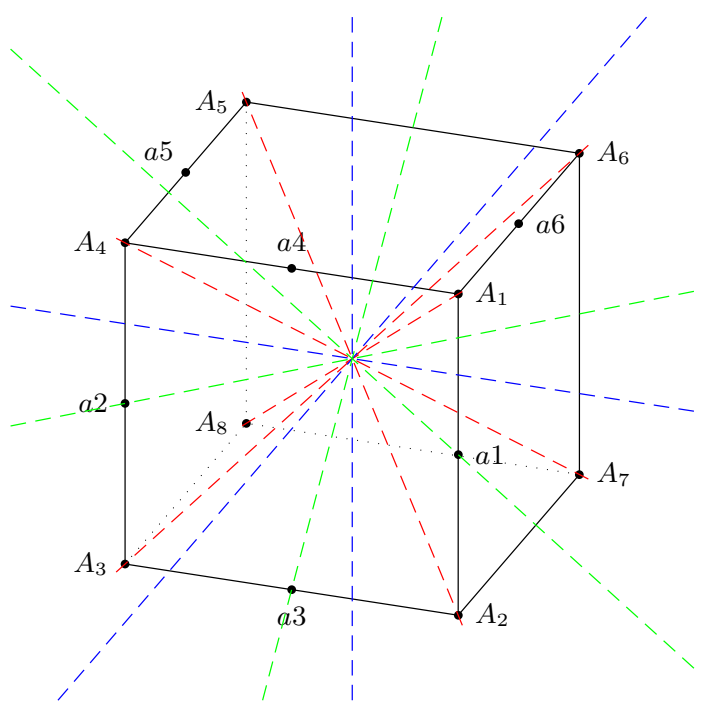

Figure 3. Symmetry axes of $\mathscr{C}_{0}$.

where the vertex, edge, and face axes are denoted respectively by $v c_{i}, e c_{j}$, and $f c_{j}$. Details can be found in Figure 3. For every rotation $g \in \mathrm{SO}(3), \mathbb{O}^{g}$ is characterized by its set of transformed axes $\left(g f c_{i}, g e c_{j}, g v c_{l}\right)$.

- The icosahedral group $\mathscr{\Phi}^{0}$ splits into

$$
g^{0}=\biguplus_{i=1}^{6} \mathrm{Z}_{5}^{f d_{i}} \uplus \biguplus_{j=1}^{10} \mathrm{Z}_{3}^{v d_{j}} \uplus \biguplus_{l=1}^{15} \mathrm{Z}_{2}^{e d_{l}},
$$

where the vertex, edge, and face axes are denoted respectively by $v d_{i}, e d_{j}$, and $f d_{j}$; the details can be found in Figure $2 \mathrm{~b}$. The vertex axes $v d_{j}$ are characterized by the vertices $D_{j}$ for $j=1 \ldots 10$.

\section{Planar subgroups.}

Cyclic subgroups. We begin with the following lemma.

Lemma A.2. For every two integers $m$ and $n$ greater than 2, and for every two axes $a$ and $b$ :

- If $a \neq b$ then $\mathrm{Z}_{n}^{a} \cap \mathrm{Z}_{m}^{b}=\mathbb{1}$.

- If $a=b$ then by setting $d:=\operatorname{gcd}(m, n)$ we will have $\mathrm{Z}_{n}^{a} \cap \mathrm{Z}_{m}^{b}=\mathrm{Z}_{d}^{a}$.

Proof. Let $g \in \mathrm{Z}_{n}^{a} \cap \mathrm{Z}_{m}^{b}$, with $a \neq b$. Both $a$ and $b$ are generated by two noncollinear eigenvectors for $g$, with eigenvalue 1 . As $\operatorname{det} g=1$ the third eigenvalue is also 1 , therefore $g=e$. Thus we have the first point of the lemma. If now we take, for example, a common rotation of $Z_{n}^{0}$ and $Z_{m}^{0}$, then this rotation corresponds to an 
angle $\theta=2 l \pi / n=2 r \pi / m$ with $r$ and $l$ integers. Thus $l m=r n$ and, setting $m=d m_{1}$ and $n=d n_{1}$, we will have $l m_{1}=r n_{1}$. As $m_{1}$ and $n_{1}$ are relatively prime, we deduce that

$$
l=\alpha n_{1} \text { and then } \theta=\frac{2 l \pi}{n}=\frac{2 \alpha \pi}{d} \in \mathrm{Z}_{d}^{0} .
$$

The converse inclusion is obvious, so we can conclude the lemma.

A direct application of Lemma A.2 to the intersection $Z_{n}^{0} \cap Z_{n}^{g}$ leads to the result:

Lemma A.3. For all integers $n$ and $m$, we set $d=\operatorname{gcd}(n, m) ;$ we have $\left[\mathrm{Z}_{n}\right] \odot\left[\mathrm{Z}_{m}\right]=$ $\left\{[\mathbb{1}],\left[\mathrm{Z}_{d}\right]\right\}$.

Dihedral subgroups. Let us consider first the intersection $\Gamma=\mathrm{D}_{n}^{0} \cap \mathrm{Z}_{m}^{a}$. As $\mathrm{D}_{n}^{0}=$ $\mathrm{Z}_{n}^{0} \biguplus_{l=1}^{n} \mathrm{Z}_{2}^{b_{l}}$ the following cases have to be considered:

- When $O z=a$, the intersection $\Gamma=\mathrm{Z}_{n}^{0} \cap \mathrm{Z}_{m}^{a}$ and one can apply Lemma A.2.

- When, for some $l$, we have $a=b_{l}$, then $Z_{n}^{0} \cap Z_{m}^{a}=\mathbb{1}$ and one has to consider $\mathrm{Z}_{2}^{b} \cap \mathrm{Z}_{m}^{a}$, which equals the identity as soon as $m$ is odd.

Lemma A.4. For every two integers $n$ and $m$, we set $d:=\operatorname{gcd}(n, m)$ and $d_{2}(m):=$ $\operatorname{gcd}(m, 2)$; then we have

$$
\left[\mathrm{D}_{n}\right] \odot\left[\mathrm{Z}_{m}\right]=\left\{[\mathbb{1}],\left[\mathrm{Z}_{d_{2}(m)}\right],\left[\mathrm{Z}_{d}\right]\right\} .
$$

Now consider the second kind of intersection:

$$
\Gamma=\mathrm{D}_{n}^{0} \cap \mathrm{D}_{m}^{g}=\left(\mathrm{D}_{n}^{0}=\mathrm{Z}_{n}^{0} \uplus \biguplus_{l=1}^{n} \mathrm{Z}_{2}^{b_{l}}\right) \cap\left(\mathrm{D}_{m}^{g}=\mathrm{Z}_{m}^{a} \uplus \biguplus_{l=1}^{m} \mathrm{Z}_{2}^{g b_{l}}\right) .
$$

The following cases have to be considered:

- When $O z=a$ and $O x=g b_{l} 0$ for some $l$ : if $d=1$ then $\Gamma=Z_{2}^{b_{0}}$, otherwise $\Gamma=\mathrm{D}_{m}^{0}$.

- When $O z=a$ and $O x \neq g b_{l}: \Gamma=Z_{d}^{0}$.

- When $O z=g b_{l}$ for some $l$ : if $n$ is even then $\Gamma=\mathrm{Z}_{2}$, otherwise $\Gamma=\mathbb{1}$. The results are the same when the primary axis of $\mathrm{D}_{m}^{g}$ coincides with a secondary axis of $\mathrm{D}_{n}^{0}$.

Lemma A.5. For all integers $n$ and $m$, we set $d:=\operatorname{gcd}(n, m)$ and

$$
d_{2}(m):=\operatorname{gcd}(m, 2), \quad d_{2}(n):=\operatorname{gcd}(n, 2), \quad d z:=\left\{\begin{array}{l}
2 \text { if } d=1, \\
1 \text { otherwise } .
\end{array}\right.
$$

Then we have $\left[\mathrm{D}_{n}\right] \odot\left[\mathrm{D}_{m}\right]=\left\{[\mathbb{1}],\left[\mathrm{Z}_{d_{2}(n)}\right],\left[\mathrm{Z}_{d_{2}(m)}\right],\left[\mathrm{Z}_{d z}\right],\left[\mathrm{Z}_{d}\right],\left[\mathrm{D}_{d}\right]\right.$. 
Clips operations on exceptional and maximum subgroups. Here we are concerned with the subgroups $\mathscr{T}^{0}, \mathrm{O}^{0}, g^{0}, \mathrm{SO}(2)^{0}$, and $\mathrm{O}(2)^{0}$. For these studies, we will use results concerning their proper subgroups [Ihrig and Golubitsky 1984]. This information is summed up in the following diagram [Auffray et al. 2011], whose arrows are to be understood as inclusion of conjugates.

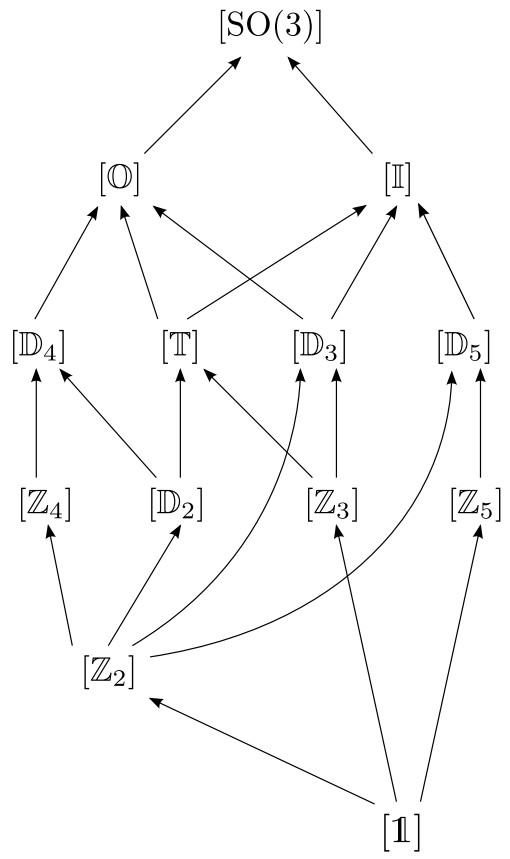

Figure 4. Exceptional subgroups in the poset of the closed subgroup of $\mathrm{SO}(3)$.

Tetrahedral subgroup. We revisit the decomposition (A.2):

$$
\mathscr{T}^{0}=\mathrm{Z}_{3}^{v t_{1}} \uplus \mathrm{Z}_{3}^{v t_{2}} \uplus \mathrm{Z}_{3}^{v t_{3}} \uplus \mathrm{Z}_{3}^{v t_{4}} \uplus \mathrm{Z}_{2}^{e t_{1}} \uplus \mathrm{Z}_{2}^{e t_{2}} \uplus \mathrm{Z}_{2}^{e t_{3}} .
$$

We begin by studying $\mathscr{T}^{0} \cap \mathrm{Z}_{n}^{a}$. As a consequence of Lemma A.2, the primary axis of $Z_{n}^{a}$ must be an edge axis or a face axis of the tetrahedron. We therefore obtain:

Lemma A.6. For every integer $n$, we set $d_{2}(n):=\operatorname{gcd}(n, 2)$ and $d_{3}(n):=\operatorname{gcd}(3, n)$; then we have $\left[\mathrm{Z}_{n}\right] \odot[\mathscr{T}]=\left\{[\mathbb{1}],\left[\mathrm{Z}_{d_{2}(n)}\right],\left[\mathrm{Z}_{d_{3}(n)}\right]\right\}$.

Now let us consider $\Gamma=\mathscr{T}^{0} \cap \mathrm{D}_{n}^{g}$. We will use the primary and secondary axes of the dihedral subgroup:

$$
\mathrm{D}_{n}^{g}=\mathrm{Z}_{n}^{a} \biguplus \mathrm{Z}_{2}^{b}
$$

We recall that the vertex axes of the tetrahedron are denoted by $v t_{i}$ and the edge axes are denoted by $e t_{j}$. It is clear that $\Gamma$ is a subgroup of $\mathscr{T}^{0}$. Furthermore: 
- As soon as $3 \mid n$ if $a=v t_{i}$ then $\Gamma=Z_{3}$ is maximal.

- When $2 \mid n$ we can find $g$ such that $a=e t_{j}$. Then if $b=e t_{j}, \Gamma=\mathrm{D}_{2}$; otherwise $\Gamma=\mathrm{Z}_{2}$.

- In any case, when we only have $b=e t_{j}$, then $\Gamma=Z_{2}$.

Finally we can deduce the lemma:

Lemma A.7. For every integer $n$ we denote $d_{2}(n):=\operatorname{gcd}(2, n)$ and $d_{3}(n):=$ $\operatorname{gcd}(3, n)$; then we have $\left[\mathrm{D}_{n}\right] \odot[\mathcal{T}]=\left\{[\mathbb{1}],\left[\mathrm{Z}_{2}\right],\left[\mathrm{Z}_{d_{3}(n)}\right],\left[\mathrm{D}_{d_{2}(n)}\right]\right\}$.

Now, for the study of $\Gamma=\mathscr{T}^{0} \cap \mathscr{T}^{g}$ the arguments will be based on $\mathscr{T}$ subgroups as well as on the axes.

- First, we can find a $g$ such that all the axes are modified; in this case $\Gamma=\mathbb{1}$.

- A rotation around a face or an edge axis can be found such that only this axis is left fixed. Then $\Gamma=Z_{2}$ or $\Gamma=Z_{3}$ depending on the fixed axis.

- If we have $\Gamma \supset \mathrm{D}_{2}$ then we can deduce that $g$ carries two edge axes onto two edge axes. After a given permutation of axes (which leaves fixed $\mathscr{T}^{0}$ ) we can suppose that $g$ leaves fixed axes $v t_{1}$ and $v t_{2}$; we then conclude that $g$ fixes also the axis $v t_{3}$ and then $\mathscr{T}^{g}=\mathscr{T}^{0}$. Thus we have here $\Gamma=\mathscr{T}^{0}$.

We deduce here the following lemma:

Lemma A.8. We have $[\mathscr{T}] \odot[\mathscr{T}]=\left\{[\mathbb{1}],\left[Z_{2}\right],\left[Z_{3}\right],[\mathscr{T}]\right.$.

Octahedral subgroup. We begin by taking back the decomposition (A.3):

$$
0^{0}=\biguplus_{i=1}^{3} \mathrm{Z}_{4}^{f c_{i}} \uplus \biguplus_{j=1}^{4} \mathrm{Z}_{3}^{v c_{j}} \uplus \biguplus_{l=1}^{6} \mathrm{Z}_{2}^{e c_{l}} .
$$

As in the case of the tetrahedron, we directly get the lemma:

Lemma A.9. For every integer $n$, we write

$$
d_{2}(n)=\operatorname{gcd}(n, 2), \quad d_{3}(n)=\operatorname{gcd}(n, 3), \quad d_{4}(n)= \begin{cases}4 & \text { if } 4 \mid n, \\ 1 & \text { otherwise. }\end{cases}
$$

Then we have $\left[\mathrm{Z}_{n}\right] \odot[0]=\left\{[\mathbb{1}],\left[\mathrm{Z}_{d_{2}(n)}\right],\left[\mathrm{Z}_{d_{3}(n)}\right],\left[\mathrm{Z}_{d_{4}(n)}\right]\right.$.

To study the clips operation with dihedral groups, we proceed in the same way as for the tetrahedron subgroup. The purpose is to examine axes of the cube and dihedral group. The arguments are about the same in each case. Therefore we will only detail the cases where $4 \nmid n, 3 \mid n$ and $n$ is odd.

- If $a=v c_{j}$ : then if $b=f c_{j}, \Gamma=\mathrm{D}_{3}$; otherwise $\Gamma=\mathrm{Z}_{3}$.

- If $a=f c_{j}$ : then if $b=f c_{j}, \Gamma=\mathrm{Z}_{2}$; otherwise $\Gamma=\mathbb{1}$.

- If $a=e c_{j}$ : then if $b=e c_{j}$ or $b=f c_{j}, \Gamma=\mathrm{Z}_{2}$. 
All these arguments leads us to the following lemma:

Lemma A.10. For every integer $n$, we set

$$
d_{2}(n):=\operatorname{gcd}(n, 2), \quad d_{3}(n):=\operatorname{gcd}(n, 3), \quad d_{4}(n):= \begin{cases}4 & \text { if } 4 \mid n, \\ 1 & \text { otherwise. }\end{cases}
$$

Then we have $\left[\mathrm{D}_{n}\right] \odot[0]=\left\{[\mathbb{1}],\left[\mathrm{Z}_{2}\right],\left[\mathrm{Z}_{d_{3}(n)}\right],\left[\mathrm{Z}_{d_{4}(n)}\right],\left[\mathrm{D}_{d_{2}(n)}\right],\left[\mathrm{D}_{d_{3}(n)}\right],\left[\mathrm{D}_{d_{4}(n)}\right]\right\}$.

Now, we take $\Gamma=\mathscr{O}^{0} \cap \mathscr{T}^{g}$ and one can observe that if $\Gamma$ is necessarily a common subgroup of $0^{0}$ and $\mathscr{T}^{g}$, then its class must contain (in the sense of the partial order) $[\mathbb{1}],\left[\mathrm{Z}_{2}\right],\left[\mathrm{Z}_{3}\right],\left[\mathrm{D}_{2}\right]$, or $[\mathscr{T}]$. After that:

- There exists a rotation $g$ around an edge axis of $\mathscr{T}^{0}$ (that is, a common face axis of the cube) such that only this axis is fixed; and then $\Gamma=Z_{2}$.

- There exists a rotation $g$ around a vertex axis of $\mathscr{T}^{0}$ (that is, a common vertex axis of the cube) such that only this axis is fixed; and then $\Gamma=Z_{3}$.

- As soon as $\Gamma \supset \mathrm{D}_{2}$, as in the tetrahedral case, we necessarily have $\Gamma=\mathscr{T}^{0}$.

We conclude here the lemma:

Lemma A.11. We have $[\mathscr{T}] \odot[\mathcal{O}]=\left\{[\mathbb{1}],\left[\mathrm{Z}_{2}\right],\left[\mathrm{Z}_{3}\right],[\mathscr{T}]\right\}$.

For the study of $\Gamma=0^{0} \cap \mathbb{O}^{g}$ we will also use arguments based on subgroups. Some results are nevertheless more subtle:

- First, there exists a rotation $g$ that fixes only one edge axis, and in that case $\Gamma=\mathrm{Z}_{2}$.

- Then there exists a rotation that leaves fixed only one vertex axis, and in that case $\Gamma=\mathrm{Z}_{3}$.

- There exists also a rotation that leaves fixed only one face axis, and no other axis is fixed. See the figure below, which illustrates the case $\Gamma=Z_{4}$.

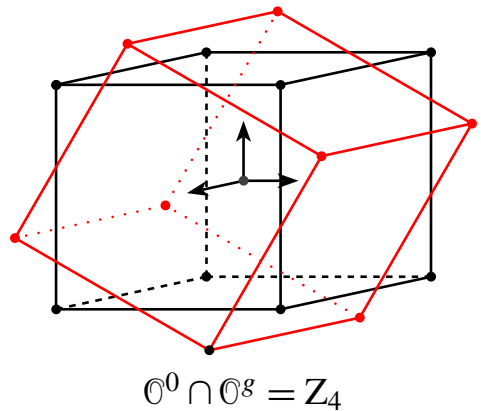

- We can also find a rotation that leaves fixed a face axis and which brings an edge axis onto a face axis. Indeed, when we take $g=\mathbf{Q}(\mathbf{i} ; \pi / 4)$ we obtain $\Gamma=Z_{4}^{\mathbf{i}} \uplus Z_{2}^{\mathbf{k}}=\mathrm{D}_{4}$; see left figure on the next page. 

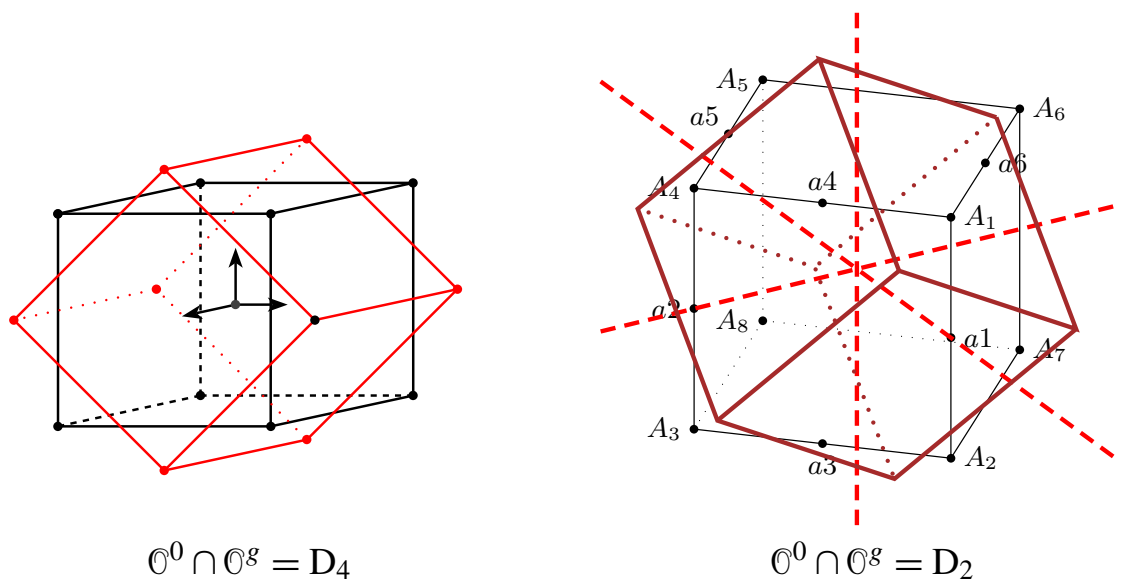

- If we take $g=\mathbf{Q}(\mathbf{k} ; \pi / 4) \circ \mathbf{Q}(\mathbf{i} ; \pi / 4)$ we directly obtain $\Gamma=\mathrm{D}_{2}$. We can exactly compute that $g f c_{3}=e c_{6}, g e c_{1}=f c_{1}$, and $g e c_{2}=e c_{5}$ and no other axes correspond; see figure above and to the right.

- If we take $g=\mathbf{Q}\left(v c_{1}, \pi\right)$ we will find $\Gamma=\mathrm{D}_{3}$ with $v c_{1}$ as primary axis and ec5 a secondary axis.

- If $\Gamma \supset \mathscr{T}$ then, necessarily, $g$ leaves fixed the three edge axes of the tetrahedron, and then $g$ will fix the cube $\mathscr{C}_{0}$; thus $\Gamma=0^{0}$.

Finally we get the lemma:

Lemma A.12. We have $[0] \odot[0]=\left\{[\mathbb{1}],\left[\mathrm{Z}_{2}\right],\left[\mathrm{D}_{2}\right],\left[\mathrm{Z}_{3}\right],\left[\mathrm{D}_{3}\right],\left[\mathrm{Z}_{4}\right],\left[\mathrm{D}_{4}\right],[\mathrm{O}]\right\}$.

Icosahedral subgroup. We take the decomposition (A.4):

$$
g^{0}=\biguplus_{i=1}^{6} \mathrm{Z}_{5}^{f d_{i}} \uplus \biguplus_{j=1}^{10} \mathrm{Z}_{3}^{v d_{j}} \uplus \biguplus_{l=1}^{15} \mathrm{Z}_{2}^{e d_{l}} .
$$

As in the previous situations, we directly get the lemma:

Lemma A.13. For every integer $n$, we set

$$
d_{2}:=\operatorname{gcd}(n, 2), \quad d_{3}:=\operatorname{gcd}(n, 3), \quad d_{5}:=\operatorname{gcd}(n, 5) .
$$

Then we have $\left[\mathrm{Z}_{n}\right] \odot[\mathscr{F}]=\left\{[\mathbb{1}],\left[\mathrm{Z}_{d_{2}}\right],\left[\mathrm{Z}_{d_{3}}\right],\left[\mathrm{Z}_{d_{5}}\right]\right\}$.

Now, for the study of $g^{0} \cap \mathrm{D}_{n}^{g}$ we again use the arguments about axes:

- If $a=f t_{j}$ or $a=v t_{j}$, then $\Gamma \in\left\{Z_{d_{3}}, Z_{d_{5}}, \mathrm{D}_{d_{3}} \mathrm{D}_{d_{5}}\right\}$.

- If $a=e t_{j}$ then $\Gamma \in\left\{Z_{d_{2}}, \mathrm{D}_{d_{2}}\right\}$.

When we argue on the secondary axis of $\mathrm{D}_{n}^{g}$, we see that we can always have $\mathrm{Z}_{2}$. Finally we get the lemma: 
Lemma A.14. For every integer $n$, we set

$$
d_{2}:=\operatorname{gcd}(n, 2), \quad d_{3}:=\operatorname{gcd}(n, 3), \quad d_{5}:=\operatorname{gcd}(n, 5) .
$$

Then we have

$$
\left.\left[\mathrm{D}_{n}\right] \odot[\mathscr{I}]=\left\{[\mathbb{1}],\left[\mathrm{Z}_{2}\right],\left[\mathrm{Z}_{d_{3}}\right],\left[\mathrm{Z}_{d_{5}}\right],\left[\mathrm{D}_{d_{2}}\right],\left[\mathrm{D}_{d_{3}}\right],\left[\mathrm{D}_{d_{5}}\right]\right\}\right\} .
$$

For the intersection $\mathscr{I}^{0} \cap \mathscr{T}^{g}$ it is clear, because of the inclusion $\mathscr{T}^{0} \subset \mathscr{I}^{0}$, that we can obtain all the classes of $[\mathscr{T}] \odot[\mathscr{T}]$. If now this intersection contains a subgroup $\mathrm{D}_{2}$, we will necessarily have

$$
\mathrm{D}_{2}=\mathrm{Z}_{2}^{\text {get }_{1}} \uplus \mathrm{Z}_{2}^{\text {get }_{3}} \uplus \mathrm{Z}_{2}^{\text {get }} \text {, }_{3},
$$

where get $_{i}$ are the three edge axis of the tetrahedron $g \mathscr{T}_{0}$. These three axes will then have to correspond to three perpendicular axes of the dodecahedron. After permutation of the axes, which leaves the dodecahedron fixed, we can suppose that these three axes are generated by the three vectors of the basis. But, then, the vertex axes of the tetrahedron will correspond to vertex axes of the embedded cube in the dodecahedron. We can then deduce that the intersection will be the whole $\mathscr{T}$ subgroup.

Now we have to study $\Gamma=g^{0} \cap \mathcal{O}^{g}$. For that, we refer to the common subgroups of [O] and [F]. Such subgroups can clearly be taken from the poset on page 203. First, it is clear that, when the cube related to $0^{g}$ is the embedded cube in the dodecahedron, we will have $\Gamma=\mathscr{T}$.

We also can find a rotation $g$ such that $\Gamma$ contains $\mathrm{D}_{3}$ : indeed, $g$ has to bring the vertex axis of the cube $v c_{1}$ onto the vertex axis of the dodecahedron $v d_{5}$ and the edge axis of the cube $e c_{5}$ onto the edge axis of the dodecahedron $e d_{7}$. With the maximality argument, we can deduce that $\Gamma=\mathrm{D}_{3}$. We now have to examine the case of $\mathrm{D}_{2}, \mathrm{Z}_{3}$, and $\mathrm{Z}_{2}$ :

- When $\Gamma \supset \mathrm{D}_{2}$, then, after permuting of the axes of the dodecahedron, we can suppose that $g$ leaves fixed the three axes of the basis vector. But these three axes are axes of rotation of order 2 of the dodecahedron. Thus, $g$ will fix the cube $\mathscr{C}_{0}$ and we can deduce that $\mathbb{O}^{g}=\mathcal{O}^{0}$. We then have $\Gamma=\mathscr{T}$.

- We can find a rotation $g$ around a vertex axis, for example, $v d_{5}$ such that $\Gamma=\mathrm{Z}_{3}$.

- As above, we can find a rotation around an edge axis, such that $\Gamma=\mathrm{Z}_{2}$.

We finally conclude with the formula:

$$
[\mathrm{O}] \odot[\mathscr{F}]=\left\{[\mathbb{1}],\left[\mathrm{Z}_{2}\right],\left[\mathrm{Z}_{3}\right],\left[\mathrm{D}_{3}\right],[\mathscr{T}]\right\} .
$$

For the intersection $\Gamma=\mathscr{I}^{0} \cap \mathscr{I}^{g}$ we will have to study the case of classes [ $\left.\mathscr{T}\right],\left[\mathrm{D}_{3}\right]$, $\left[\mathrm{D}_{5}\right],\left[\mathrm{D}_{2}\right],\left[\mathrm{Z}_{3}\right],\left[\mathrm{Z}_{5}\right]$, and $\left[\mathrm{Z}_{2}\right]$ : 
- When $\Gamma \supset \mathscr{T}$ or $\Gamma \supset \mathrm{D}_{2}$, it then contains all the three second-order rotations around each base axis, which will be three edge axes of the dodecahedron. Thus we can deduce that $g$, after permutation of these axes, leaves fixed three perpendicular axes, and then $g$ leaves fixed $\mathscr{l}^{0}$; finally $\Gamma=\mathscr{I}$.

- There exists a rotation $g$ around an edge axis so that $\Gamma=Z_{2}$. The same argument leads us to $Z_{3}$ and $Z_{5}$.

- If we take $g$ to be the second-order rotation around the axis $v d_{3}$, we can compute that this rotation only leaves fixed the axes $v d_{3}, e d_{6}, e d_{8}$, and $e d_{15}$, and then $\Gamma=\mathrm{D}_{3}$.

- If we take $g$ to be the second-order rotation around the face axis $f d_{1}$ we can also compute that it only leaves fixed the axes $f d_{1}, e d_{7}, e d_{11}, e d_{12}$, and $e d_{14}$, thus $\Gamma=\mathrm{D}_{5}$.

\section{References}

[Abud and Sartori 1983] M. Abud and G. Sartori, "The geometry of spontaneous symmetry breaking”, Ann. Phys. 150:2 (1983), 307-372.

[Auffray 2008] N. Auffray, "Décomposition harmonique des tenseurs: méthode spectrale", $C$. $R$. Mécanique 336:4 (2008), 370-375.

[Auffray et al. 2011] N. Auffray, B. Kolev, and M. Petitot, "Invariant-based approach to symmetry class detection”, preprint, 2011. arXiv 1111.0861

[Auffray et al. 2013] N. Auffray, H. Le Quang, and Q.-C. He, "Matrix representations for 3D straingradient elasticity", J. Mech. Phys. Solids (2013).

[Backus 1970] G. Backus, “A geometrical picture of anisotropic elastic tensors”, Rev. Geophys. 8:3 (1970), 633-671.

[Bóna et al. 2004] A. Bóna, I. Bucataru, and M. A. Slawinski, "Characterization of elasticity-tensor symmetries using SU(2)”, J. Elasticity 75:3 (2004), 267-289.

[Bredon 1972] G. E. Bredon, Introduction to compact transformation groups, Pure and Applied Mathematics 46, Academic Press, New York, 1972.

[Chadwick et al. 2001] P. Chadwick, M. Vianello, and S. C. Cowin, "A new proof that the number of linear elastic symmetries is eight”, J. Mech. Phys. Solids 49:11 (2001), 2471-2492.

[dell'Isola et al. 2009] F. dell'Isola, G. Sciarra, and S. Vidoli, “Generalized Hooke's law for isotropic second gradient materials", Proc. R. Soc. Lond. A 465:2107 (2009), 2177-2196.

[dell'Isola et al. 2012] F. dell'Isola, P. Seppecher, and A. Madeo, "How contact interactions may depend on the shape of Cauchy cuts in $N$ th gradient continua: approach 'à la D'Alembert"', $Z$. Angew. Math. Phys. 63:6 (2012), 1119-1141.

[Forest 1998] S. Forest, "Mechanics of generalized continua: construction by homogenization", $J$. Phys. (France) IV 8:4 (1998), 39-48.

[Forest et al. 2011] S. Forest, N. M. Cordero, and E. P. Busso, "First vs. second gradient of strain theory for capillarity effects in an elastic fluid at small length scales", Comput. Mater. Sci. 50:4 (2011), 1299-1304. 
[Forte and Vianello 1996] S. Forte and M. Vianello, "Symmetry classes for elasticity tensors", J. Elasticity 43:2 (1996), 81-108.

[Forte and Vianello 1997] S. Forte and M. Vianello, "Symmetry classes and harmonic decomposition for photoelasticity tensors", Int. J. Eng. Sci. 35:14 (1997), 1317-1326.

[Geymonat and Weller 2002] G. Geymonat and T. Weller, "Classes de symétrie des solides piézoélectriques”, C. R. Acad. Sci. Paris Sér. I Math. 335:10 (2002), 847-852.

[Golubitsky et al. 1988] M. Golubitsky, I. Stewart, and D. G. Schaeffer, Singularities and groups in bifurcation theory, vol. 2, Applied Mathematical Sciences 69, Springer, New York, 1988.

[Gu and He 2011] S. T. Gu and Q. C. He, "Interfacial discontinuity relations for coupled multifield phenomena and their application to the modeling of thin interphases as imperfect interfaces", $J$. Mech. Phys. Solids 59:7 (2011), 1413-1426.

[Herman 1945] B. Herman, "Some theorems of the theory of anisotropic media", C. R. (Doklady) Acad. Sci. URSS (N. S.) 48 (1945), 89-92.

[Ihrig and Golubitsky 1984] E. Ihrig and M. Golubitsky, "Pattern selection with O(3) symmetry", Physica D 13:1-2 (1984), 1-33.

[Jerphagnon et al. 1978] J. Jerphagnon, D. Chemla, and R. Bonneville, "The description of the physical properties of condensed matter using irreducible tensors", Adv. Phys. 27:4 (1978), 609650 .

[Le Quang and He 2011] H. Le Quang and Q.-C. He, "The number and types of all possible rotational symmetries for flexoelectric tensors", Proc. R. Soc. Lond. A 467:2132 (2011), 2369-2386.

[Le Quang et al. 2012] H. Le Quang, N. Auffray, Q.-C. He, and G. Bonnet, "Symmetry groups and symmetry classes for sixth-order tensors", preprint, 2012. Submitted to Proc. R. Soc. Lond. A.

[Lebée and Sab 2011] A. Lebée and K. Sab, "A bending-gradient model for thick plates, I: Theory", Int. J. Solids Struct. 48 (2011), 2878-2888.

[Mindlin 1964] R. D. Mindlin, "Micro-structure in linear elasticity", Arch. Ration. Mech. Anal. 16 (1964), 51-78.

[Mindlin 1965] R. D. Mindlin, "Second gradient of strain and surface-tension in linear elasticity", Int. J. Solids Struct. 1:4 (1965), 417-438.

[Spencer 1970] A. J. M. Spencer, "A note on the decomposition of tensors into traceless symmetric tensors”, Int. J. Eng. Sci. 8 (1970), 475-481.

[Sternberg 1994] S. Sternberg, Group theory and physics, Cambridge University Press, Cambridge, 1994.

[Thionnet and Martin 2006] A. Thionnet and C. Martin, "A new constructive method using the theory of invariants to obtain material behavior laws", Int. J. Solids Struct. 43:2 (2006), 325-345.

[Toupin 1962] R. A. Toupin, "Elastic materials with couple-stresses", Arch. Ration. Mech. Anal. 11 (1962), 385-414.

[Triantafyllidis and Bardenhagen 1996] N. Triantafyllidis and S. Bardenhagen, "The influence of scale size on the stability of periodic solids and the role of associated higher order gradient continuum models", J. Mech. Phys. Solids 44:11 (1996), 1891-1928.

[Zheng and Boehler 1994] Q.-S. Zheng and J.-P. Boehler, "The description, classification, and reality of material and physical symmetries", Acta Mech. 102:1-4 (1994), 73-89.

Received 16 May 2012. Revised 2 Oct 2012. Accepted 2 Dec 2012. 
MARC OLIVE: molive@cmi.univ-mrs.fr

Laboratoire d'Analyse, Topologie, Probabilités, Centre de Mathématiques et Informatique,

CNRS, UMR 7353, Aix-Marseille Université, Technopôle Château-Gombert, 39 rue F. Joliot-Curie, 13453 Marseille, France

NICOLAS AUFFRAY: Nicolas.Auffray@univ-mlv.fr

Laboratoire Modélisation et Simulation Multi Echelle, Université Paris-Est, CNRS, UMR 8208, 5 boulevard Descartes, 77454 Marne-la-Vallée, France 


\title{
A DIRECT APPROACH TO NONLINEAR SHELLS WITH APPLICATION TO SURFACE-SUBSTRATE INTERACTIONS
}

\author{
MiroslaV ŠILHAVÝ
}

\begin{abstract}
The paper develops a direct, intrinsic approach to the equilibrium equations of bodies coated by a thin film with a nonlinear shell like structure. The forms of the equations in the reference and actual configurations are considered. The equations are shown to coincide with those obtained by using coordinate systems on the film or on the thin shell.
\end{abstract}

\section{Introduction}

This paper presents equilibrium equations of the system consisting of a bulk solid and attached boundary film. The film is assumed to exhibit resistance to flexural deformations in that its energy is a nonlinear function of the boundary first-order deformation gradient and of a second-order tensor that represents a suitable version of its curvature in the deformed state. Such a theory was developed by Steigmann and Ogden [1997a; 1997b; 1999] in dimensions $n=2$ (plane deformations) and $n=3$ (full three-dimensional deformations). The case $n=2$ has also been treated by Fried and Todres [Fried and Todres 2005]. The cited works by Steigmann and Ogden generalize the situation in [Gurtin and Murdoch 1975; Podio-Guidugli 1988; Podio-Guidugli and Vergara-Caffarelli 1990; Steigmann and Li 1995; Steinmann 2008], where the film is modeled as a nonlinear membrane, i.e., its energy is assumed to depend only on the first surface deformation gradient.

Steigmann and Ogden used a variational principle to derive the equilibrium equations (among other things) and to show that they coincide with those of thin nonlinear shells; see [Sanders 1963; Cohen and De Silva 1966; Naghdi 1971; Pietraszkiewicz 1989].

The purpose of the present paper is to derive a direct, index-free, form of the balance equations. This approach allows a more unified understanding of the underlying mechanics than the coordinate-based approach, where one is typically forced to cover the manifold with coordinate patches.

The formalism I adopt is different from the intrinsic approaches in [Delfour and Zolésio 1997] and [Favata and Podio-Guidugli 2011]. The basic feature of

MSC2010: primary 74K25; secondary 74K 35 .

PACS2010: primary 68.35.Md; secondary 68.35.Gy.

Keywords: thin films, nonlinear shells, surface geometry, variation of surface energy, equilibrium equations. 
the present work is the treatment of the surface quantities as tensors in the threedimensional space and not just on the tangent space to the shell at the given point. This allows for a fully tensorial form of the equilibrium equations. Both the referential and the actual configurations are considered. The main results are the intrinsic form of the first variation of the surface energy Proposition 5.2 and the associated equilibrium equations (15), the fully intrinsic form (17) of the effective second-order stress tensor, the spatial intrinsic form of the equilibrium equations Proposition 6.1, and the tangential and normal components of the equilibrium equations Proposition 6.2. Up to the last mentioned item, no coordinate system is invoked to derive the results. However, for reasons of comparison with the existing coordinate approaches, in Section 7 I give the coordinate form of the main results and show that they coincide with those obtained by different methods.

As for the intrinsic tensor calculus, only tensors in euclidean space will be employed, of orders $0,1,2$, and 3. Tensors of orders 0 and 1 are scalars and vectors from $\mathrm{R}^{n}$ ( $n \geq 2$; typically $n=2$ or $n=3$ ). Second-order tensors are either $\mathrm{R}$ valued bilinear forms on $\mathrm{R}^{n} \times \mathrm{R}^{n}$ or linear transformations from $\mathrm{R}^{n}$ to $\mathrm{R}^{n}$; we do not distinguish these two interpretations graphically. The set of all second-order tensors is denoted by Lin. The third-order tensors are mostly interpreted as $\mathrm{R}^{n}$ valued bilinear forms on $\mathrm{R}^{n} \times \mathrm{R}^{n}$. The set of all third-order tensors is denoted by $T$.

\section{Geometry of deformation of a coated body}

We identify the material points of the body with their positions $\mathbf{x}$ in a reference configuration $\Omega \subset \mathrm{R}^{n}$, where $n \geq 2$ is arbitrary but in applications $n=2$ or $n=3$. We assume that $\Omega$ is a bounded open set with sufficiently smooth boundary $\partial \Omega$ with the unit outer normal $๓$. We consider the bulk solid to be coated with an elastic surface $\mathcal{S} \subset \partial \Omega$. We assume that $\mathcal{S}$ is a relatively open subset of $\partial \Omega$ with a smooth boundary $\partial \mathcal{S}$ without corners.

The deformation of the coated body is described by a sufficiently smooth map from the closure $\mathrm{cl} \Omega$ of $\Omega$ so that the deformation $\mathrm{y}$ of the coating, i.e., the restriction of $\mathbf{y}$ to the closure $\operatorname{cl} \mathcal{S}$ of $\mathcal{S}$, is well defined and sufficiently smooth.

The deformation $\mathbf{y}$ of the bulk body is described by the bulk deformation gradient

$$
\mathbf{F}=\nabla \mathbf{y}
$$

which is assumed to exist, be continuous, and of positive determinant, at every point of $\mathrm{cl} \Omega$. Here $\nabla$ indicates the gradient with respect to the position in the reference configuration and at a given point of $\operatorname{cl} \Omega, \mathbf{F}$ is interpreted as a linear transformation from $\mathrm{R}^{n}$ to $\mathrm{R}^{n}$.

The surface deformation $\searrow$ of the coating is described by the surface deformation gradient and by the referential version of the curvature tensor of the coating in the 
deformed configuration to be defined below. For the referential surface $\mathcal{S}$ of the coating we introduce the curvature tensor $\mathbb{b}$ (also called the Weingarten tensor), defined by

$$
\llcorner=\nabla \infty,
$$

where $\nabla$ denotes the surface gradient. We here adopt the following convention for the surface differentiation of maps with values is a finite-dimensional vector space $V$ defined on a manifold $\mathcal{M}$ of dimension $k$ in $\mathrm{R}^{n}$ : if $f: \mathcal{M} \rightarrow V$ is a smooth map then for every $\mathbf{x} \in \mathcal{M}$ the surface gradient $\nabla f(\mathbf{x})$ of $f$ at $\mathbf{x}$ is a linear map from the whole space $\mathrm{R}^{n}$ to $V$ which satisfies $\nabla f(\mathbf{x}) \mathbb{P}(\mathbf{x})=\nabla f(\mathbf{x})$, where $\mathbb{P}(\mathbf{x})$ is a projection from $\mathrm{R}^{n}$ onto the tangent space $\operatorname{Tan}(\mathcal{M}, \mathbf{x})$ of $\mathcal{M}$ at $\mathbf{x}$, and

$$
\lim _{\substack{\overline{\mathbf{x}} \rightarrow \mathbf{x} \\ \mathbf{\mathbf { x }} \in \mathcal{M}, \overline{\mathbf{x}} \neq \mathbf{x}}} \frac{|f(\overline{\mathbf{x}})-f(\mathbf{x})-\nabla f(\mathbf{x})(\overline{\mathbf{x}}-\mathbf{x})|}{|\overline{\mathbf{x}}-\mathbf{x}|}=0 .
$$

This convention differs from the alternative view [Federer 1969, Subsection 3.1.22; Gurtin and Murdoch 1975; Gurtin 2000], where the surface gradient at the given point is interpreted as a linear transformation $\operatorname{from} \operatorname{Tan}(\mathcal{M}, \mathbf{x})$ to $V$. The latter is just the restriction of our $\nabla f(\mathbf{x})$ to $\operatorname{Tan}(\mathcal{M}, \mathbf{x})$. Below we apply the same convention to the derivatives of the response functions for the surface energy with respect to the surface deformation gradient and curvature. Our convention has the advantage that the surface gradient at different points of $\mathcal{M}$ is an element of the same linear space and one can thus iterate the procedure to define the second surface gradient $\nabla^{2} f(\mathbf{x})$ of $f$ at $x \in \mathcal{M}$ as the surface gradient of the surface gradient. Thus $\nabla^{2} f(\mathbf{x})=\nabla(\nabla f)(\mathbf{x})$ and we interpret $\nabla^{2} f(\mathbf{x})$ as a bilinear transformation from $\mathrm{R}^{n} \times \mathrm{R}^{n}$ to $V$, defined by

$$
\nabla^{2} f(\mathbf{x})(\mathbf{a}, \mathbf{b})=\nabla(\nabla f \mathbf{b}) \mathbf{a}
$$

for every $\mathbf{a}, \mathbf{b} \in \mathrm{R}^{n}$. A comparison with [Murdoch and Cohen 1979/80] shows that the second gradient as defined there is similarly the present second gradient restricted to $\operatorname{Tan}(\mathcal{M}, \mathbf{x}) \times \operatorname{Tan}(\mathcal{M}, \mathbf{x})$. In [Steigmann and Ogden 1999] the notion of the second gradient is employed in the special case of the second surface gradient. We shall see below that this notion of the second surface gradient coincides with the present one also as far as the definition domain is concerned. We note that the bilinear map $\nabla^{2} f(\mathbf{x})$ is generally nonsymmetric, but its restriction to $\operatorname{Tan}(\mathcal{M}, \mathbf{x}) \times$ $\operatorname{Tan}(\mathcal{M}, \mathbf{x})$ is symmetric. If $V=\mathrm{R}^{n}$, i.e., if $f$ is a scalar function, we identify the linear transformation $\nabla f(\mathbf{x})$ from $\mathrm{R}^{n}$ to $\mathrm{R}$ with an equally denoted element of $\mathrm{R}^{n}$ via the identification $\nabla f(\mathbf{x}) \mathbf{a}=\nabla f(\mathbf{x}) \cdot \mathbf{a}$ for each $\mathbf{a} \in \mathrm{R}^{n}$. The equation $\nabla f(\mathbf{x}) \mathbb{P}(\mathbf{x})=\nabla f(\mathbf{x})$ then reduces to $\mathbb{P}(\mathbf{x}) \nabla f(\mathbf{x})=\nabla f(\mathbf{x})$, i.e., $\nabla f(\mathbf{x})$ is an element of the tangent space to $\mathcal{M}$ at $\mathbf{x}$. Similarly, the second gradient $\nabla^{2} f(\mathbf{x})$ is identified with a second-order tensor in Lin via $\nabla^{2} f(\mathbf{x})(\mathbf{a}, \mathbf{b})=\mathbf{a} \cdot \nabla^{2} f(\mathbf{x}) \mathbf{b}$ for each $\mathbf{a}, \mathbf{b} \in \mathrm{R}^{n}$. 
We refer to [Šilhavý 2011, Appendix A and B] for more details on the present conventions on the derivatives and gradients.

We define the surface deformation gradient $F$ of $y$ by

$$
F=\nabla y .
$$

At the given point $\mathbf{x}$ of $\mathcal{S}$, $F$ is a second-order tensor on $\mathrm{R}^{n}$ which is assumed to map $\operatorname{Tan}(\mathcal{S}, \mathbf{x})$ onto $\operatorname{Tan}(\overline{\mathcal{S}}, \mathbb{y}(\mathbf{x}))$, where $\overline{\mathcal{S}}=\mathbb{y}(\mathcal{S})$ is the actual configuration of the coating. We denote by $\mathbb{P}$ the orthogonal projection onto the tangent space to $\mathcal{S}$ and by $\overline{\mathbb{P}}$ the orthogonal projection onto the tangent space to $\overline{\mathcal{S}}$. Then we have

$$
F P=F, \quad \bar{P} F=F .
$$

The tensor $\mathbb{F}$ is always noninvertible. However, we denote by $\mathbb{F}^{-1}$ the pseudoinverse, which at a given point of $\mathcal{S}$ is a linear transformation from $\mathrm{R}^{n}$ to $\mathrm{R}^{n}$ satisfying

$$
F^{-1} \leftleftarrows=P, \quad F^{-1}=\bar{P} .
$$

Then $F^{-1}$ always exists and is determined uniquely. If, for a given point of $\mathcal{S}, \mathbf{F}$ is the bulk deformation gradient at that point, then

$$
F=\mathbf{F} P, \quad F^{-1}=\mathbf{F}^{-1} \bar{P},
$$

where $\mathbf{F}^{-1}$ is the inverse of $\mathbf{F}$ in the standard sense.

We assume that the response of the coating depends on the first and second deformation gradients, but on the second deformation gradient $\nabla^{2} y$ only through a combination that can be regarded as the curvature tensor of the deformed configuration $\overline{\mathcal{S}}$ viewed from the reference configuration. That is, we introduce a bilinear form $\mathbb{K}$ which is identified with an equally denoted second-order tensor by

$$
\mathbb{R}(\mathbf{a}, \mathbf{b})=\bar{\pi} \cdot \nabla^{2} \mathbb{Y}(\mathbb{P} \mathbf{a}, \mathbb{P} \mathbf{b})
$$

for every $\mathbf{a}, \mathbf{b} \in \mathrm{R}^{n}$, where

$$
\bar{\infty}=\frac{\operatorname{cof}[\infty 0}{\mid \operatorname{cof}[50 \mid}
$$

is the unit outer normal to $\overline{\mathcal{S}}$. Here cof $F$ is the cofactor tensor of $F$. If for any map $\mathrm{B}$ on $\mathrm{R}^{n} \times \mathrm{R}^{n}$ we introduce the symbol $\mathrm{B} \circ(\mathrm{P}, \mathbb{P})$ to denote the map on $\mathrm{R}^{n} \times \mathrm{R}^{n}$ given by

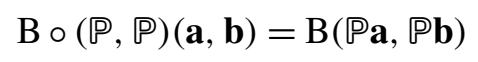

for any $\mathbf{a}, \mathbf{b} \in \mathrm{R}^{n}$, then we have

$$
\mathbb{K}=\overline{\mathfrak{N}} \cdot \nabla^{2} \mathfrak{y} \circ(\mathbb{P}, \mathbb{P})
$$

and

$$
\mathbb{K}=\mathbb{K} \circ(\mathbb{P}, \mathbb{P}) .
$$


Also, when viewed as a second-order tensor, $\mathbb{K}$ satisfies

$$
P R P=K .
$$

It is useful to note that the curvature $\bar{\square}=\bar{\nabla} \overline{0}$ of the surface $\overline{\mathcal{S}}$ is

$$
\overline{\mathrm{L}}=-\mathbb{F}^{-\mathrm{T}} \mathfrak{K F}^{-1} \text {. }
$$

Here $\bar{\nabla}$ is the surface gradient on $\overline{\mathcal{S}}$, i.e., the surface gradient as defined above, but for maps on $\mathcal{M}=\overline{\mathcal{S}}$, and $\mathbb{F}^{-\mathrm{T}}:=\left(\mathbb{F}^{-1}\right)^{\mathrm{T}}$ where $\mathrm{T}$ denotes the transposition.

If $\mathcal{M} \subset \mathrm{R}^{n}$ is a smooth manifold of dimension $k$ and if

$$
\text { Q }: \mathcal{M} \rightarrow \operatorname{Lin}\left(\mathrm{R}^{n}, V\right)
$$

is a map on $\mathcal{M}$ with values in the space $\operatorname{Lin}\left(\mathrm{R}^{n}, V\right)$ of linear transformations from $\mathrm{R}^{n}$ to a finite-dimensional inner product space $V$, we define the surface divergence div $@: \mathcal{M} \rightarrow V$ by

$$
\mathrm{a} \cdot \operatorname{diov} Q=\operatorname{tr}\left(\nabla\left(\mathbb{Q}^{\mathrm{T}} \mathrm{a}\right)\right)
$$

for each $a \in V$ where the transpose

$$
\mathbb{Q}^{\mathrm{T}}: \mathcal{M} \rightarrow \operatorname{Lin}\left(V, \mathrm{R}^{n}\right)
$$

is defined by $\mathbf{b} \cdot \mathbb{Q}^{\mathrm{T}} \mathbf{a}=\mathbb{Q} \mathbf{b} \cdot \mathbf{a}$ for each $\mathbf{b} \in \mathrm{R}^{n}$ and $\mathbf{a} \in V$. It follows directly from the definition that if $\mathrm{a}: \mathcal{M} \rightarrow V$ then

$$
\operatorname{div}\left(Q^{\mathrm{T}} \mathbf{a}\right)=\mathbf{a} \cdot \operatorname{div} Q+Q \cdot \nabla \mathbf{a},
$$

where

$$
\text { Q. } \nabla \mathbf{a}:=\operatorname{tr}\left(Q^{\mathrm{T}} \nabla \mathbf{a}\right) \text {. }
$$

If $V=\mathrm{R}$, we identify $\mathbb{Q}: \mathcal{M} \rightarrow \operatorname{Lin}\left(\mathrm{R}^{n}, \mathrm{R}\right)$ with a vector field $\mathbb{q}: \mathcal{M} \rightarrow \mathrm{R}^{n}$ by $Q \mathbf{a}=\mathfrak{q} \cdot \mathbf{a}$ for each $\mathbf{a} \in \mathrm{R}^{n}$ and define the divergence of $q$ to be the divergence of Q; thus div q is a scalar field defined by

$$
\text { di̊v } q=\operatorname{tr}(\nabla q)
$$

and (3) can be rewritten as a $\operatorname{div} Q=\operatorname{div}\left(Q^{\mathrm{T}} \mathrm{a}\right)$ for each $\mathrm{a} \in V$.

We say that $\mathbb{Q}$ as in (2) is superficial if $\mathbb{Q}=\mathbb{Q}$. In particular if $\mathbb{Q}: \mathcal{M} \rightarrow$ $\operatorname{Lin}\left(\mathrm{R}^{n}, \mathrm{R}\right)$ and $\mathbb{q}: \mathcal{M} \rightarrow \mathrm{R}^{n}$ are related as above, $\mathbb{Q}$ is superficial if and only if $\mathbb{q}$ is tangential, i.e., $\mathbb{q}$ is an element of the tangent space at every point: $\mathbb{P} q=\mathbb{q}$.

We define the relative boundary $\partial \mathcal{M}$ of $\mathcal{M}$ by $\partial \mathcal{M}=\operatorname{cl} \mathcal{M} \backslash \mathcal{M}$ where $\operatorname{cl} \mathcal{M}$ is the closure of $\mathcal{M}$ in $\mathrm{R}^{n}$. We assume that $\partial \mathcal{M}$ is sufficiently smooth. We denote by 00 the relative normal to $\partial \mathcal{M}$. This is a map which, at a given point of $\partial \mathcal{M}$, is an element to the tangent space to $\mathcal{M}$ defined, e.g., by $\boldsymbol{c o s}^{\prime}=\nabla \varphi /|\nabla \varphi|$ where $\varphi$ is a function defined locally on $\mathcal{M}$ such that the equation $\varphi=0$ expresses locally $\partial \mathcal{M}$. We here assume that $\mathcal{M}$ can be extended to a smooth manifold of dimension 
$k$ in $\mathrm{R}^{n}$ which contains $\operatorname{cl} \mathcal{M}$; this makes the tangent space to $\mathcal{M}$ defined also at the points of $\partial \mathcal{M}$ and the equation $\varphi=0$ makes sense. The surface divergence theorem asserts that if @ as in (2) is superficial then

$$
\int_{\mathcal{M}} \operatorname{diov} Q d \mathcal{H}^{k}=\int_{\partial \mathcal{M}} \text { Qmo } d \mathcal{H}^{k-1} \text {. }
$$

Here $\mathcal{H}^{k}$ is the $k$-dimensional area measure on $\mathcal{M}$ and $\mathcal{H}^{k-1}$ the $(k-1)$-dimensional area measure on $\partial \mathcal{M}$.

Furthermore, the surface Piola transformation asserts that if $\mathbb{Q}$ is as in (2) is superficial, if $\boldsymbol{\Phi}: \mathcal{M} \rightarrow \overline{\mathcal{M}}:=\boldsymbol{\Phi}(\mathcal{M})$ is a diffeomorphism and if $\overline{\mathbb{Q}}$ is a field defined on $\overline{\mathcal{M}}$ by

$$
\overline{\mathbb{Q}}=\mathfrak{j}^{-1} @ F^{\mathrm{T}},
$$

where $\mathfrak{j}$ is the jacobian of $\boldsymbol{\Phi}, \mathbb{F}:=\nabla \boldsymbol{\Phi}$, then $\overline{\mathbb{Q}}$ is superficial and

$$
\overline{\operatorname{div}} \bar{Q}=j^{-1} \operatorname{diov} Q
$$

where $\overline{\text { div }}$ is the surface divergence on $\overline{\mathcal{M}}$, i.e., the surface divergence as defined above, but for fields defined on $\overline{\mathcal{M}}$. Below we need the cases $\mathcal{M}=\mathcal{S}$ and $\mathcal{M}=\partial \mathcal{S}$. Moreover, we shall employ $V=\mathrm{R}, V=\mathrm{R}^{n}$ and $V=$ Lin, i.e., Q will be a vector field, second-order tensor field and third-order tensor field. We refer to [Marsden and Hughes 1983, Chapter 1] for abstract formulations of Stokes' theorem and surface Piola transformation on manifolds from which the present euclidean cases follow.

\section{Constitutive assumptions}

We assume that the bulk body is made of a nonlinear hyperelastic material with the bulk stored energy $\tilde{f}: \operatorname{Lin}_{+} \rightarrow \mathrm{R}$, where $\operatorname{Lin}_{+}$is the set of all second-order tensors with positive determinant. For a given deformation $\mathbf{y}: \operatorname{cl} \Omega \rightarrow \mathrm{R}^{n}$ the stored energy field is given by the constitutive equation

$$
f(\mathbf{x})=\tilde{f}(\mathbf{F}(\mathbf{x})), \quad \mathbf{x} \in \operatorname{cl} \Omega,
$$

where $\mathbf{F}$ is the bulk deformation gradient. For the coating $\mathcal{S}$ we assume that for each $\mathbf{x} \in \mathcal{S}$ we have a surface stored energy function $\tilde{\mathbf{f}}_{\mathbf{x}}: D_{\mathbf{x}} \rightarrow \mathrm{R}^{n}$ where $D_{\mathbf{x}}$ is the set of all pairs $(\mathbb{F}, \mathbb{K}) \in \operatorname{Lin} \times$ Lin such that $F \mathbb{P}(\mathbf{x})=\mathbb{F}$ and $\mathbb{K}$ is symmetric and satisfies $\mathbb{P}(\mathbf{x}) \mathbb{K} \mathbb{P}(\mathbf{x})=\mathbb{K}$. For a given deformation $\mathbb{y}: \mathcal{S} \rightarrow \mathrm{R}^{n}$ the field of superficial stored energy $\mathrm{f}$ on $\mathcal{S}$ is given by the constitutive equation

$$
\mathfrak{f}(\mathbf{x})=\tilde{\mathfrak{f}}_{\mathbf{x}}(\mathbb{F}(\mathbf{x}), \mathbb{K}(\mathbf{x})), \quad \mathbf{x} \in \mathcal{S},
$$

where $\mathbb{F}$ and $\mathbb{K}$ are defined in Section 2. We note that the response function for the superficial stored energy depends on $\mathbf{x}$ since we are forced to assume that the 
domain $D_{\mathbf{x}}$ is different for different $\mathbf{x} \in \mathcal{S}$. The same applies for the derivatives of $\tilde{\mathbf{f}}_{\mathbf{x}}$. However, below we simplify the notation and suppress the dependence of $\tilde{\mathbf{f}}_{\mathbf{X}}$ on $\mathbf{x}$ and write simply $\tilde{\mathbf{f}}$ in place of $\tilde{\mathfrak{f}}_{\mathbf{X}}$. The same convention applies for the derivatives of $\tilde{\mathbf{f}}$. The constitutive assumption (7) is employed in [Steigmann and Ogden 1999] in a coordinate form, who refer to [Hilgers and Pipkin 1992; Cohen and De Silva 1966] for earlier employments of the same hypothesis. In $n=2$ (plane deformations of the bulk body), equivalent hypotheses have been made in [Steigmann and Ogden 1997a; 1997b; Fried and Todres 2005]. See Section 7 (below) for the coordinate version of this assumption for $n=3$.

Following [Steigmann and Ogden 1999], we also treat the coating $\mathcal{S}$ as a general second grade material, i.e., we treat the superficial stored energy as a function of the first and second surface gradients. More precisely, we introduce a third-order tensor $\mathbb{G}$ interpreted as an $\mathrm{R}^{n}$-valued bilinear form on $\mathrm{R}^{n} \times \mathrm{R}^{n}$ given by

$$
G(\mathbf{a}, \mathbf{b})=\nabla^{2} y(P \mathbf{a}, \mathbb{P} \mathbf{b})
$$

for all $\mathbf{a}, \mathbf{b} \in \mathrm{R}^{n}$, note that

$$
\mathbb{K}(\mathbf{a}, \mathbf{b})=\overline{\mathbb{0}} \cdot \mathbb{G}(\mathbf{a}, \mathbf{b}),
$$

and introduce a response function $\hat{\mathrm{f}} \equiv \hat{\mathfrak{f}}_{\mathbf{x}}: E_{\mathbf{x}} \rightarrow \mathrm{R}$ related to $\tilde{\mathrm{f}}$ by

$$
\hat{\mathbf{f}}(\varpi, G)=\tilde{\mathfrak{f}}(『, \mathbb{K})
$$

where $\mathbb{G}$ and $\mathbb{K}$ are related by (8). The domain $E_{\mathbf{x}}$ of $\hat{\mathbf{f}}$ consists of all pairs (臣, G) $\in$ Lin $\times T$, where $T$ is the space of all $\mathrm{R}^{n}$-valued bilinear forms on $\mathrm{R}^{n} \times \mathrm{R}^{n}$, satisfying

$$
F=F \mathbb{P}, \quad \mathbb{G}=\mathbb{G} \circ(\mathbb{P}, \mathbb{P}) .
$$

The field $\mathrm{f}$ is then given by the constitutive equation

$$
\mathbf{f}(\mathbf{x})=\hat{f}(\mathbb{F}(\mathbf{x}), \mathbb{G}(\mathbf{x})), \quad x \in \mathcal{S} .
$$

\section{The total energy}

The total energy $F$ of the bulk body plus the coating is assumed in the form

$$
F(y)=E_{b}(\mathbf{y})+E_{c}(\mathbf{y})+W(y)
$$

for each deformation $\mathbf{y}: \operatorname{cl} \Omega \rightarrow R^{n}$, where $E_{b}(y)$ is the internal energy of the bulk body, $E_{c}(y)$ is the internal energy of the coating, and $W(y)$ is the potential energy of the loads. Here

$$
\mathrm{E}_{\mathrm{b}}(\mathbf{y})=\int_{\Omega} f d \mathcal{L}^{n},
$$


where $f$ is given by the constitutive equation (6) and $\mathcal{L}^{n}$ is the $n$-dimensional volume in $\mathrm{R}^{n}$,

$$
\mathrm{E}_{\mathrm{c}}(\mathbf{y}) \equiv \mathrm{E}_{\mathrm{c}}(\mathrm{y})=\int_{\mathcal{S}} \mathbf{f} d \mathcal{H}^{n-1}
$$

where $\mathrm{f}$ is given by the constitutive equation (7) and $\mathcal{H}^{n-1}$ is the $(n-1)$-dimensional area on $\partial \Omega$, and

$$
\mathrm{W}(\mathbf{y})=-\int_{\Omega} \mathbf{y} \cdot \mathbf{b} d \mathcal{L}^{n}-\int_{\partial \Omega} \mathbf{y} \cdot \mathbf{s} d \mathcal{H}^{n-1}
$$

here $\mathbf{b}: \Omega \rightarrow \mathrm{R}^{n}$ is a prescribed body force and $\mathbf{s}: \partial \Omega \rightarrow \mathrm{R}^{n}$ is a prescribed surface traction on the boundary of the body.

We assume that the response functions for the bulk and surface energies are sufficiently smooth and define the first variation $\delta \mathrm{F}(\mathbf{y}, \mathbf{v})$ of the total energy corresponding to the variation $\mathbf{v}: \operatorname{cl} \Omega \rightarrow \mathrm{R}^{n}$ by

$$
\delta \mathrm{F}(\mathbf{y}, \mathbf{v})=\left.\frac{d \mathrm{~F}(\mathbf{y}+t \mathbf{v})}{d t}\right|_{t=0} .
$$

We define the first variations $\delta \mathrm{E}_{\mathrm{b}}(\mathbf{y}, \mathbf{v})$ and $\delta \mathrm{E}_{\mathrm{c}}(\mathbf{y}, \mathbf{v})$ of the internal energies and the first variation $\delta \mathrm{W}(\mathbf{y}, \mathbf{v})$ of the potential energy of loads analogously.

\section{The first variation of total energy and the Euler Lagrange equations}

Proposition 5.1. For every deformation $\mathbf{y}: \operatorname{cl} \Omega \rightarrow \mathrm{R}^{n}$ and every variation of deformation $\mathbf{v}: \operatorname{cl} \Omega \rightarrow \mathrm{R}^{n}$, we have

$$
\delta \mathrm{E}_{\mathrm{b}}(\mathbf{y}, \mathbf{v})=-\int_{\Omega} \mathbf{v} \cdot \operatorname{div} \mathbf{S} d \mathcal{L}^{n}+\int_{\partial \Omega} \mathbf{v} \cdot \mathbf{S} \propto 0 \mathcal{H}^{n-1}
$$

where $\mathbf{S}$ is the bulk referential stress given by the constitutive equation

$$
\mathbf{S}(\mathbf{x})=\tilde{\mathbf{S}}(\mathbf{F}(\mathbf{x})), \quad \text { with } \mathbf{x} \in \operatorname{cl} \Omega, \tilde{\mathbf{S}}=\partial_{\mathbf{F}} \tilde{f} .
$$

Furthermore,

$$
\delta \mathrm{W}(\mathbf{y}, \mathbf{v})=-\int_{\Omega} \mathbf{v} \cdot \mathbf{b} d \mathcal{L}^{n}-\int_{\partial \Omega} \mathbf{v} \cdot \mathbf{s} d \mathcal{H}^{n-1}
$$

This is standard.

Proposition 5.2. We have

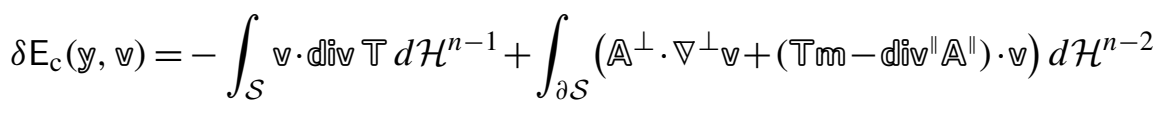

for each deformation $\mathrm{y}: \mathrm{cl} \mathcal{S} \rightarrow \mathrm{R}^{n}$ and for each variation $\mathbb{\vee}: \mathrm{cl} \mathcal{S} \rightarrow \mathrm{R}^{n}$ of deformation, where $\mathcal{H}^{n-2}$ is the (n-2)-dimensional area measure on $\partial \mathcal{S}$,

$$
\checkmark=S-(\operatorname{div} \mathbb{A}) P
$$


with $\mathbb{S}$ and $\mathbb{A}$ the referential surface stress and the referential surface couple stress given by the constitutive equations

$$
\mathfrak{S}(\mathbf{x})=\hat{\mathbb{S}}(\mathbb{F}(\mathbf{x}), \mathbb{G}(\mathbf{x})), \quad \mathbb{A}(\mathbf{x})=\hat{\mathbb{A}}(\mathbb{F}(\mathbf{x}), \mathbb{G}(\mathbf{x})), \quad \mathbf{x} \in \mathrm{cl} \mathcal{S},
$$

with

$$
\hat{\mathfrak{S}}=\partial_{\mathbb{F}} \hat{\mathbf{f}}, \quad \hat{\mathbb{A}}=\partial_{\mathbb{G}} \hat{\mathfrak{f}},
$$

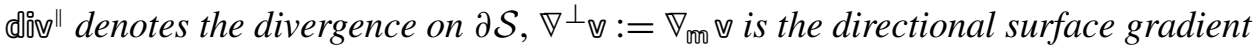
of $\mathbb{\vee}$ in the direction of the normal $\mathrm{m}$, and $\mathbb{A}^{\perp}$ and $\mathbb{A}^{\|}$are fields on $\partial \mathcal{S}$ given by

$$
\mathbb{A}^{\perp}=\mathbb{A}(\infty 0, \infty), \quad \mathbb{A}^{\|} \mathbf{a}=\mathbb{A}(\mathbb{P} \| \mathbf{a}, \infty)
$$

for each $\mathbf{a} \in \mathrm{R}^{n}$, where $\mathbb{P} \|$ is the orthogonal projection from $\mathrm{R}^{n}$ onto the tangent space of $\partial \mathcal{S}$ at the given point.

We here recall that the response function $\hat{\mathrm{f}}$ is defined on the domain $E_{\mathbf{x}}$ which consists of all pairs $(\mathbb{F}, \mathbb{G}) \in \operatorname{Lin} \times T$ such that (9) hold. Thus for a given point of $\mathcal{S}$, the domain of $\hat{\mathbb{f}}$ is a linear subspace of the product $\operatorname{Lin} \times T$. The partial derivatives of $\hat{f}$ in (12) follow our convention about derivatives on submanifolds of an euclidean space and interpret the total derivative (differential) $D \hat{\mathbf{f}}$ of $\hat{\mathbf{f}}$ as an element of the space $\operatorname{Lin} \times T$, which satisfies

$$
\Pi D \hat{\mathfrak{f}}=\hat{\mathfrak{f}}
$$

where $\Pi$ is the orthogonal projection from $\operatorname{Lin} \times T$ onto $E_{\mathbf{x}}$. The value $\mathrm{D} \hat{\mathrm{f}}$ is a pair in Lin $\times T$ and we write $D \hat{\mathbb{f}}=\left(\partial_{\mathbb{F}} \hat{\mathbf{f}}, \partial_{\mathbb{R}} \hat{\mathbf{f}}\right)$ with $\partial_{\mathbb{F}} \hat{\mathbf{f}} \in \operatorname{Lin}, \partial_{\mathbb{K}} \hat{\mathbf{f}} \in T$ for the "components" of D $\hat{f}$. Equation (13) and the definition of $E_{\mathbf{x}}$ gives

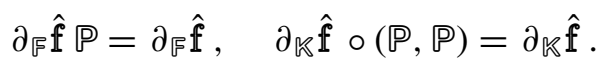

Proof. The definition of the variation gives

$$
\delta \mathrm{E}_{\mathrm{c}}(\mathbb{Y}, \mathbb{v})=\int_{\mathcal{S}}\left(\mathbb{S} \cdot \mathbb{\nabla} \mathbb{v}+\mathbb{A} \cdot\left(\nabla^{2} \mathbb{v}(\mathbb{P}, \mathbb{P})\right)\right) d \mathcal{H}^{n-1} .
$$

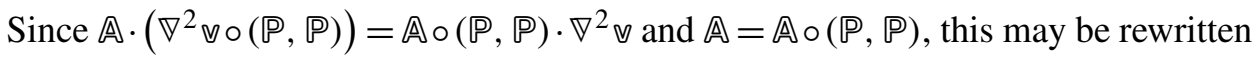
as

$$
\delta \mathrm{E}_{\mathrm{c}}(\mathrm{g}, \mathbb{v})=\int_{\mathcal{S}}\left(\mathbb{S} \cdot \nabla \mathbb{v}+\mathbb{A} \cdot \nabla^{2} \mathbb{v}\right) d \mathcal{H}^{n-1}
$$

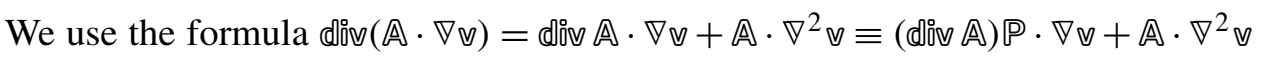
[see (4)] and employ the surface divergence theorem noting that $\mathbb{A}$ is superficial to obtain

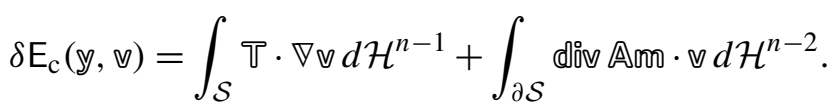


Next we use the formula $\operatorname{div}\left(\nabla^{\mathrm{T}} \mathbb{v}\right)=(\operatorname{div} \nabla) \cdot \vee+\nabla \cdot \nabla \vee$ and employ the surface divergence theorem to obtain

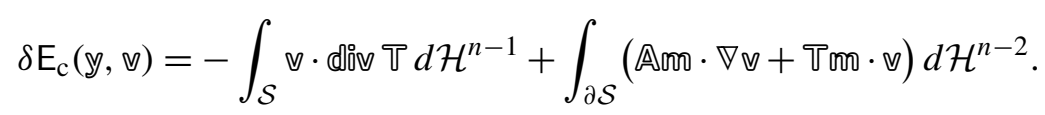

The second integral on the right hand side is further transformed as follows. We write $\nabla \vee=\nabla^{\perp} \vee+\nabla^{\|} \vee$ where $\nabla^{\|}$denotes the surface gradient relative to $\partial \mathcal{S}$ to obtain

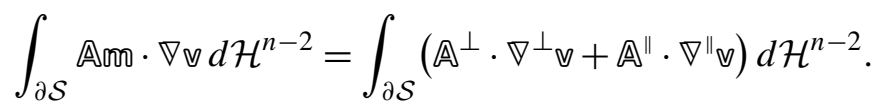

Recalling that $\partial^{2} \mathcal{S}=\varnothing$, we use the surface divergence theorem to obtain

$$
\int_{\partial \mathcal{S}} \operatorname{diov}^{\|}\left(\mathbb{A}^{\| T} \cdot \mathbb{v}\right) d \mathcal{H}^{n-2}=0 .
$$

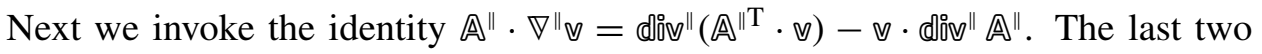
relations provide

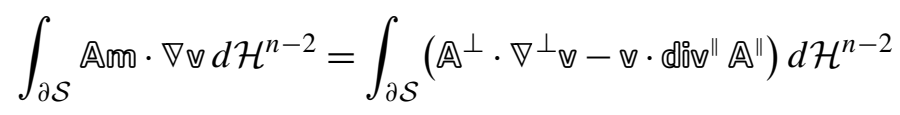

and this reduces (14) to (10).

Proposition 5.3. If $\delta \mathrm{F}(\mathbf{y}, \mathbf{v})=0$ for a given deformation $\mathbf{y}: \mathrm{cl} \Omega \rightarrow \mathrm{R}^{n}$ and all variations of deformation $\mathbf{v}: \mathrm{cl} \Omega \rightarrow \mathrm{R}^{n}$, we have the equations

$$
\begin{aligned}
& \operatorname{div} \mathbf{S}+\mathbf{b}=\mathbf{0} \quad \text { on } \Omega, \\
& \mathbf{S} 0 \mathbf{0}=\mathbf{s} \\
& \operatorname{div} \tau+p=\mathbf{0} \\
& \mathbb{\Delta}^{\perp}=0, \quad \nabla m-\operatorname{div} \| \mathbb{A}^{\|}=\mathbf{0} \text { on } \partial \mathcal{S},
\end{aligned}
$$

where

$$
\mathbb{P}=\mathbf{s}-\mathbf{S} \curvearrowleft .
$$

If $n=3$ and ↔ is the counterclockwise unit tangent vector to $\partial \mathcal{S}$ then

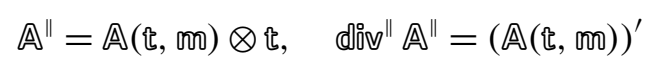

where' denotes the derivative with respect to the arc length parameter on $\partial \mathcal{S}$.

Proof. Collecting the expressions in Propositions 5.1 and 5.2, we obtain

$$
\begin{aligned}
& \delta \mathrm{F}(\mathbf{y}, \mathbf{v})=-\int_{\Omega} \mathbf{v} \cdot(\operatorname{div} \mathbf{S}+\mathbf{b}) d \mathcal{L}^{n}+\int_{\partial \Omega \backslash \mathcal{S}} \mathbf{v} \cdot\left(\mathbf{S}_{\bullet}-\mathbf{s}\right) d \mathcal{H}^{n-1} \\
& -\int_{\mathcal{S}} \mathbb{v} \cdot(\operatorname{div} 『-\mathbf{S} \oplus+\mathbf{s}) d \mathcal{H}^{n-1}
\end{aligned}
$$

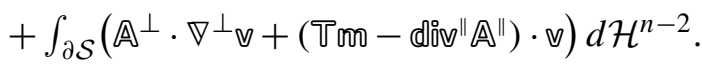


We first consider all variations $\mathbf{v}$ with compact support contained in the open set $\Omega$, then the integrals over $\partial \Omega \backslash \mathcal{S}$, over $\mathcal{S}$ and over $\partial \mathcal{S}$ vanish and the arbitrariness of $\mathbf{v}$ gives (15) 1 . With this knowledge the volume integral in (16) disappears. We then consider variations $\mathbf{v}$ such that $\mathbf{v}=0$ on $\operatorname{cl} \mathcal{S}$; then the integrals over $\mathcal{S}$ and $\partial \mathcal{S}$ vanish and the arbitrariness of the values of $\mathbf{v}$ on $\partial \Omega \backslash \mathcal{S}$ gives $(15)_{2}$. With this knowledge, also the integral over $\Omega \backslash \mathcal{S}$ disappears from (16). Then we consider variations $\vee=\mathbf{v} \mid \mathrm{cl} \mathcal{S}$ such that $\vee$ has compact support in the relatively open set $\mathcal{S}$. This gives (15) $)_{3}$ and the integral over $\mathcal{S}$ in (16) disappears. We are thus left with only the integral over $\partial \mathcal{S}$ in (16). Since the variations $\nabla^{\perp} \mathbb{\vee}$ and $\mathbb{} \mid \partial \mathcal{S}$ can be chosen independently, we obtain $(15)_{4}$.

We recall that our basic response function for the surface energy was $\tilde{\mathbf{f}}$, expressing the surface stored energy as a function of $\mathbb{F}$ and $\mathbb{K}$. The next proposition expresses the tensor $\checkmark$ occurring in (10) and $(15)_{3}$ in terms of the derivatives of $\tilde{\mathbf{f}}$.

Proposition 5.4. We have the following relation for the tensor $『$ in (11):

$$
\mho=\partial_{\mathbb{F}} \tilde{\mathbf{f}}-\operatorname{div}\left(\overline{\bar{x}} \otimes \mathbb{F} \partial_{\mathbb{R}} \tilde{\mathbf{f}}\right) \mathbb{F}^{-\mathrm{T}} .
$$

Proof. Let us show that the partial derivatives of the functions $\hat{\mathbf{f}}$ and $\tilde{\mathrm{f}}$ are related by

$$
\partial_{\mathbb{F}} \hat{\mathbf{f}}=\partial_{\mathbb{F}} \tilde{\mathbf{f}}-\bar{\varpi} \otimes\left(\mathbb{F}^{-1} \mathbb{G} \cdot \partial_{\mathbb{K}} \tilde{\mathbf{f}}\right), \quad \partial_{\mathbb{G}} \hat{\mathbf{f}}=\bar{\varpi} \otimes \partial_{\mathbb{K}} \tilde{\mathbf{f}}
$$

at the corresponding arguments, where $\mathbb{F}^{-1} \mathbb{G} \cdot \partial_{\mathbb{R}} \tilde{\mathfrak{f}}$ is a vector satisfying

$$
\mathbf{a} \cdot\left(\mathbb{F}^{-1} \mathbb{G} \cdot \partial_{\mathbb{K}} \tilde{\mathbf{f}}\right)=\mathbb{R} \cdot \partial_{\mathbb{K}} \tilde{\mathbf{f}}
$$

for all $\mathbf{a} \in \mathrm{R}^{n}$, where $\mathrm{R}$ is a second-order tensor satisfying

$$
\mathbb{R}(\mathbf{p}, \mathbf{q})=\mathbf{a} \cdot \mathbb{F}^{-1} \mathbb{G}(\mathbf{p}, \mathbf{q})
$$

for all $\mathbf{p}, \mathbf{q} \in \mathrm{R}^{n}$. Indeed, we interpret $\bar{x}$ as a function of $F$ determined locally uniquely by the equations $\vec{F}^{\mathrm{T}} \overline{\bar{x}}=\mathbf{0},|\bar{\infty}|=1$. This functional interpretation of $\bar{\pi}$ makes $\mathbb{K}$ a function of $\mathbb{G}$ and $\bar{x}$. Differentiating the relation

$$
\hat{\mathbf{f}}(\mathbb{F}, \mathbb{G})=\tilde{\mathbf{f}}(\mathbb{F}, \overline{\mathfrak{m}}(\mathbb{F}) \cdot \mathbb{G})
$$

with respect to $\mathbb{G}$ we obtain $(18)_{2}$. To obtain $(18)_{1}$, we first note that interpreting $\overline{0}$ as a function of $\mathbb{F}$, we have the relation

$$
\partial_{\text {F }} \overline{\overline{0}} \mathbf{A}=-F^{-\mathrm{T}} \mathbf{A}^{\mathrm{T}} \overline{\bar{\pi}}
$$

for each $\mathbf{A} \in$ Lin, where we interpret $\partial_{\mathbb{F}} \overline{\bar{D}}$ as a linear transformation from Lin to $\mathrm{R}^{n}$. Differentiating (19) with respect to $\mathbb{F}$ in the direction of $\mathbf{A} \in$ Lin and using the above relation for the derivative on $\overline{\tilde{n}}$, we obtain

$$
\partial_{\mathbb{F}} \hat{\mathbf{f}} \cdot \mathbf{A}=-\left(\mathbb{F}^{-\mathrm{T}} \mathbf{A}^{\mathrm{T}} \overline{\boldsymbol{m}} \cdot \mathbb{G}\right) \cdot \partial_{\mathbb{K}} \tilde{\mathbf{f}}+\partial_{\mathbb{F}} \tilde{\mathbf{f}} \cdot \mathbf{A},
$$


where for any vector a the symbol a $\cdot \mathbb{G}$ denotes a second-order tensor defined by

$$
(\mathbf{a} \cdot \mathbb{G})(\mathbf{p}, \mathbf{q})=\mathbf{a} \cdot \mathbb{G}(\mathbf{p}, \mathbf{q})
$$

for any $\mathbf{p}, \mathbf{q} \in \mathrm{R}^{n}$. We have the following rearrangements

$$
\begin{aligned}
\left(\mathbb{F}^{-\mathrm{T}} \mathbf{A}^{\mathrm{T}} \overline{\overline{0}} \cdot \mathbb{G}\right) \cdot \partial_{\mathbb{K}} \tilde{\mathbf{f}} & =\left(\mathbb{F}^{-\mathrm{T}} \mathbf{A}^{\mathrm{T}} \overline{\bar{n}}\right) \cdot\left(\mathbb{G} \cdot \partial_{\mathbb{R}} \tilde{\mathfrak{f}}\right) \\
& =\left(\mathbf{A}^{\mathrm{T}} \overline{\overline{0}}\right) \cdot\left(\mathbb{F}^{-1} \mathbb{G} \cdot \partial_{\mathbb{K}} \tilde{\mathbf{f}}\right) \\
& =\left(\overline{\bar{\infty}} \otimes\left(\mathbb{F}^{-1} \mathbb{G} \cdot \partial_{\mathbb{K}} \tilde{\mathbf{f}}\right)\right) \cdot \mathbf{A}
\end{aligned}
$$

which reduces (20) to

$$
\partial_{\mathbb{F}} \hat{\mathbf{f}} \cdot \mathbf{A}=-\left(\overline{\overline{0}} \otimes\left(\mathbb{F}^{-1} \mathbb{G} \cdot \partial_{\mathbb{K}} \tilde{\mathbf{f}}\right)\right) \cdot \mathbf{A}+\partial_{\mathbb{F}} \tilde{\mathbf{f}} \cdot \mathbf{A}
$$

and the arbitrariness of $\mathbf{A}$ gives (18) 1 .

Using relations (18) one finds from the definition (11) of $\nabla$ that

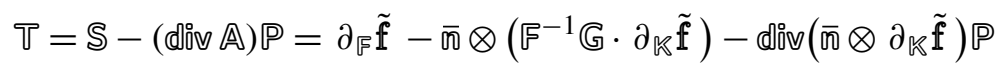

and the proof of (17) is completed by noting the following easily provable identity

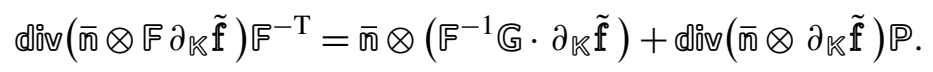

\section{The spatial form of equilibrium equations}

The spatial form of the equilibrium equations (i.e., that on the deformed configuration of the film) to be derived below admits a splitting into the tangential and normal components with the tangential component given by a second-order equation and the normal component a fourth-order equation with the iterated surface divergence.

Proposition 6.1. Assume that the stored energy $\tilde{\mathrm{f}}$ is objective in the sense that

$$
\tilde{\mathfrak{f}}(\mathbf{Q} F, \mathbb{K})=\tilde{\mathfrak{f}}(\mathbb{F}, \mathbb{K})
$$

for all orthogonal tensors $\mathbf{Q}$ and all arguments $\mathbb{F}$ and $\mathbb{R}$ from the domain of $\tilde{\mathfrak{f}}$. Then $(15)_{3}$ is equivalent to

$$
\overline{\operatorname{div}} \bar{\nabla}+\mathbf{j}^{-1} \mathbb{p}=\mathbf{0},
$$

where $\mathrm{j}=|\operatorname{cof} F|$ is the jacobian of the transformation $\mathbb{y}: \mathcal{S} \rightarrow \overline{\mathcal{S}}:=\mathbb{g}(\mathcal{S})$, बiv is the divergence on the actual configuration $\overline{\mathcal{S}}$, and

$$
\bar{\nabla}=j^{-1} \nabla F^{\mathrm{T}} \equiv \overline{\mathbb{N}}-\overline{\mathbb{L}} \overline{\mathbb{M}}-\overline{\mathbb{N}} \otimes(\overline{\mathbb{P}} \overline{\text { div }} \overline{\mathbb{M}}),
$$

where

$$
\overline{\mathbb{N}}=\mathfrak{j}^{-1} \partial_{\mathbb{F}} \tilde{\mathbf{f}} \mathbb{F}^{\mathrm{T}}, \quad \overline{\mathbb{M}}=\mathfrak{j}^{-1} \mathbb{F} \partial_{\mathbb{K}} \tilde{\mathbf{f}} \mathbb{F}^{\mathrm{T}}
$$


and $\overline{\mathrm{L}}=\bar{\nabla} \overline{\mathrm{w}}$ is the curvature of the deformed configuration of the film. Equations $(15)_{4}$ are equivalent to

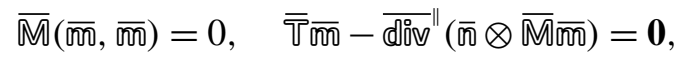

where $\overline{\bar{n}}$ is the unit normal to $\partial \overline{\mathcal{S}}$ in the tangent space to $\overline{\mathcal{S}}$ and $\overline{\text { div }} \|$ is the divergence relative to $\partial \overline{\mathcal{S}}$. If $n=3$, then $(24)_{2}$ reads

$$
\bar{\nabla} \overline{N_{0}}-(\bar{\infty} \overline{\mathbb{M}}(\overline{\mathfrak{L}}, \bar{\infty}))^{\prime}=\mathbf{0} \text {, }
$$

where $\overline{\mathbb{B}}$ is the counterclockwise unit vector tangent to $\partial \overline{\mathcal{S}}$ and the superscript ${ }^{\prime}$ denotes the derivative with respect to the arc length parameter on $\partial \overline{\mathcal{S}}$.

Here and below in this section we distinguish the objects related to the deformed configuration by a superimposed bar. Here $\overline{\mathbb{N}}$ is the normal stress and $\overline{\mathbb{N}}$ the couple stress in the film.

Proof. Note first that a standard argument based on the objectivity implies that $\overline{\mathbb{N}}$, given by $(23)_{1}$, is a symmetric tensor. Furthermore, the definition $(23)_{1}$ immediately implies that $\overline{\mathbb{N}} \overline{\mathbb{N}}=\mathbf{0}$ and $\overline{\mathbb{N}} \overline{\mathbb{P}}=\overline{\mathbb{N}}$, where $\bar{P}$ is the orthogonal projection from $\mathrm{R}^{n}$ onto the tangent space to $\overline{\mathcal{S}}$, which by the symmetry implies that $\overline{\mathbb{P}} \overline{\mathbb{N}}=\overline{\mathbb{N}}$.

We note further that if $\bar{\nabla}$ is given by $(22)_{1}$ then (21) is equivalent to $(15)_{3}$ by the surface version of the Piola transformation (5). To obtain the equivalent form $(22)_{2}$, we note that by (17) we have

$$
\overline{\mathbb{V}}=\overline{\mathbb{N}}-\mathfrak{j}^{-1} \operatorname{din}\left(\bar{\infty} \otimes F \partial_{\mathbb{R}} \tilde{\mathbf{f}}\right) \bar{P} .
$$

Employing the surface version of the Piola transformation once more and invoking the formula for the divergence of a tensor product, we find that

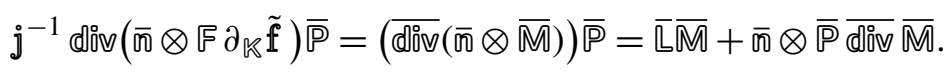

The insertion into (25) yields $(22)_{2}$.

Equation $(24)_{1}$ is clearly equivalent to the first equation in $(15)_{4}$. To obtain the equivalence of $(24)_{2}$ and the second equation in $(15)_{4}$, we employ the Piola transformation to the passage from $\partial \mathcal{S}$ to $\partial \overline{\mathcal{S}}$. We note that the jacobian of this transformation is

$$
j=j \mid F^{-T} \text { เั0 } \mid
$$

and the unit normal $\overline{\bar{m}}$ to $\partial \overline{\mathcal{S}}$ is given by

$$
\overline{\bar{m}}=F^{-\mathrm{T}} \text { mo } / \mid F^{-\mathrm{T}} \text { ஸั0 } \mid \text {. }
$$

The basic relation (5) of the Piola transformation is then

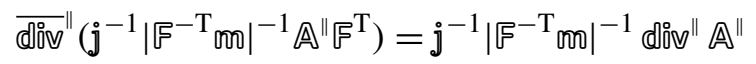


which reduces the second equation in $(15)_{4}$ to

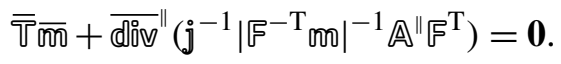

Recalling $(18)_{2}$, we note that

$$
\mathbb{A}=\bar{\pi} \otimes \partial_{\mathbb{R}} \tilde{f}
$$

and we use this in the following computation:

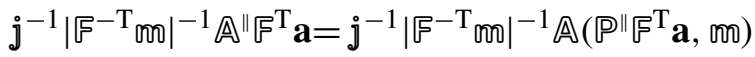

$$
\begin{aligned}
& =j^{-1} \mid \mathbb{A}\left(\mathbb{P}^{\|} \mathbb{F}^{\mathrm{T}} \mathbf{a}, \boldsymbol{F}^{\mathrm{T}} \overline{\overline{0}}\right) \\
& =\mathfrak{j}^{-1} \mid \mathbb{A}\left(F^{\mathrm{T}} \overline{\mathrm{P}} \| \mathbf{a}, \boldsymbol{F}^{\mathrm{T}} \overline{\overline{\mathbf{m}}}\right) \\
& =\overline{\mathbb{M}}(\overline{\mathbb{P}} \| \mathbf{a}, \bar{\infty}) \bar{\infty} \\
& =\overline{\mathbb{M}}((\overline{\mathbb{P}}-\bar{m} \otimes \bar{m}) \mathbf{a}, \bar{m}) \bar{m}
\end{aligned}
$$

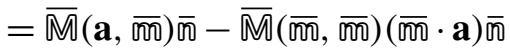

$$
\begin{aligned}
& =\overline{\mathbb{M}}(\mathbf{a}, \bar{\cdots}) \bar{\infty} \text {, }
\end{aligned}
$$

where we have used $(24)_{1}$. Thus we conclude that

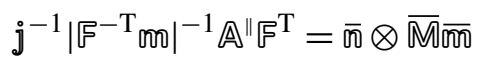

and the above computation also shows that $\overline{\mathbb{M}} \overline{\widetilde{m}}$ is a tangential vector on $\partial \overline{\mathcal{S}}$, i.e., $\overline{\mathrm{P}} \| \overline{\mathrm{M}} \overline{\bar{\infty}}=\overline{\mathbb{M}} \overline{\mathrm{N}}$. Thus we have $(24)_{2}$.

Proposition 6.2. The tangential and normal components of (21) read

$$
\left.\begin{array}{c}
\overline{\mathbb{P}} \overline{\operatorname{div}}(\overline{\mathbb{N}}-\overline{\mathbb{L}} \overline{\mathbb{M}})-\overline{\mathbb{L}} \overline{\operatorname{div}} \overline{\mathbb{M}}+\mathfrak{j}^{-1} \mathbb{P}^{\|}=\mathbf{0}, \\
\overline{\operatorname{div}}(\overline{\mathbb{P}} \overline{\operatorname{div}} \overline{\mathbb{M}})+\overline{\mathbb{L}} \cdot \overline{\mathbb{N}}-\overline{\mathbb{L}}^{2} \cdot \overline{\mathbb{M}}-\mathfrak{j}^{-1} \mathbb{P}^{\perp}=0,
\end{array}\right\}
$$

where

$$
\mathbb{P}^{\|}=\overline{\mathbb{P}} \mathbb{P}, \quad \mathbb{P}^{\perp}=\overline{\boldsymbol{m}} \cdot \mathbb{P} .
$$

The tangential and normal components of $(24)_{2}$ read

$$
\left.\begin{array}{c}
(\overline{\mathbb{N}}-2 \overline{\mathbb{L}} \overline{\mathbb{M}}) \overline{\bar{m}}=\mathbf{0}, \\
\bar{m} \cdot \overline{\operatorname{div}} \overline{\mathbb{M}}+\overline{\operatorname{div}} \|(\bar{\Phi} \| \overline{\mathbb{M}} \bar{m})=0
\end{array}\right\}
$$

on $\partial \overline{\mathcal{S}}$. If $n=3$ then $(27)_{2}$ reads

$$
\overline{\pi_{0}} \cdot \overline{\operatorname{div}} \overline{\mathbb{M}}+(\overline{\mathbb{M}}(\overline{\mathfrak{L}}, \overline{\bar{N}}))^{\prime}=0 \text {. }
$$

We recall that $\overline{\mathbb{N}}$ and $\overline{\mathbb{N}}$ depend on the first and second gradients of the surface deformation, which gives that $(26)_{1}$ is of the second order in the deformation and $(26)_{2}$ of the fourth order. 
Proof. To obtain the tangential component of (21), we use the identity

$$
\overline{\mathrm{P}} \overline{\operatorname{div}} \bar{\nabla}=\overline{\mathrm{P}}^{2} \overline{\operatorname{div}} \overline{\mathrm{V}}=\overline{\mathrm{P}} \overline{\operatorname{div}}(\overline{\mathrm{P}} \overline{\mathrm{V}})-\overline{\mathrm{P}} \overline{\mathrm{P}} \overline{\mathrm{P}} \overline{\mathrm{V}}
$$

where we note that employing the formula $\overline{\mathbb{P}} \overline{\mathbb{N}}=\overline{\mathbb{N}}$ we obtain

$$
\overline{\mathrm{P}}=\overline{\mathbb{N}}-\overline{\mathrm{L}} \overline{\mathbb{M}} .
$$

Furthermore, differentiating $\bar{\Phi}=\mathbf{1}-\bar{\oplus} \otimes \bar{\infty}$ one finds that the directional gradient in $\overline{\mathcal{S}}$ of $\bar{\Phi}$ in the direction a satisfies

$$
\bar{P}_{\bar{\nabla}} \bar{P} \mathbf{b}=-\bar{L} \mathbf{a}(\overline{0} \cdot \mathbf{b})
$$

for each $\mathbf{a}, \mathbf{b} \in \mathrm{R}^{n}$; it follows that

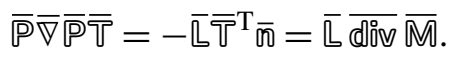

Inserting (30) and (31) into (29), one obtains (26) 1 .

To obtain the normal component of (21), we employ the identity

$$
\overline{\mathfrak{n}} \cdot \overline{\operatorname{div}} \bar{\nabla}=\overline{\operatorname{div}}\left(\bar{\nabla}^{\mathrm{T}} \overline{\mathrm{n}}\right)-\bar{\nabla} \cdot \overline{\mathrm{L}} \text {. }
$$

Since $\overline{\mathbb{N}}$ is symmetric, we have $\overline{\mathbb{N}}^{T} \overline{\overline{0}}=\overline{\mathbb{N}} \overline{\mathbb{N}}=\mathbf{0}$; combining with $\bar{\complement} \overline{\overline{0}}=\mathbf{0}$, we find

$$
\bar{\nabla}^{\mathrm{T}} \overline{\mathrm{D}}=-\overline{\mathrm{P}} \overline{\operatorname{div}} \overline{\mathbb{M}} .
$$

Also

$$
\bar{\nabla} \cdot \bar{L}=\overline{\mathbb{N}} \cdot \bar{L}-\bar{L} \overline{\mathbb{M}} \cdot \bar{L} .
$$

Inserting (33) and (34) into (32), we obtain (26) 2 .

To obtain (27), we invoke the identity

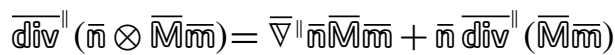

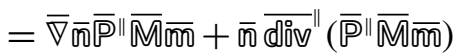

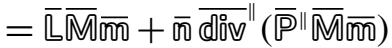

and combining with the second expression in (22) we conclude that $(24)_{2}$ is equivalent to

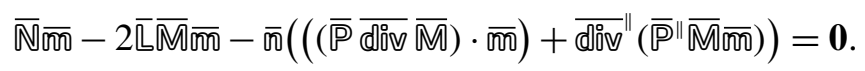

Taking the tangential and normal components, we obtain (27). 


\section{Coordinate expressions}

For the purpose of comparison with the existing literature, we now establish the component expressions of the main formulas.

Throughout this section we use the convention that the Greek indices $\alpha, \beta, \gamma$ run from 1 to $n-1$ while the Latin indexes $i, j, k$ run from 1 to $n$. We use the Einstein summation convention for repeated indices.

We assume that $\mathcal{S}$ is parametrized by a map $\Phi: D \rightarrow \mathcal{S}$, where $D \subset \mathrm{R}^{n-1}$ and note that we can express the general maps $m$ defined on $\mathcal{S}$ as functions $\tilde{m}$ of the variables $\left(\theta^{1}, \ldots, \theta^{n-1}\right) \in D$ of the parametrization $\boldsymbol{\Phi}\left(\theta^{1}, \ldots, \theta^{n-1}\right)$. The maps $m$ and $\tilde{m}$ are related by $\tilde{m}=m \circ \boldsymbol{\Phi}$ but below we denote both $\tilde{m}$ and $m$ by the same letter $m$. A Greek subscript following a comma denotes the partial differentiation with respect to the corresponding variable in the collection $\left(\theta^{1}, \ldots, \theta^{n-1}\right)$.

We denote by $\mathbf{e}_{i} \equiv \mathbf{e}^{i}$ the canonical basis in $\mathrm{R}^{n}$ and introduce the coordinate vectors $\Theta_{\alpha}$ in the tangent space of $\mathcal{S}$ by

$$
\Theta_{\alpha}=\boldsymbol{\Phi}_{, \alpha} .
$$

We denote by $\AA^{\beta}$ the dual basis in the tangent space, satisfying

$$
\mathrm{e}_{\alpha} \cdot \mathrm{e}^{\beta}=\delta_{\alpha}^{\beta}
$$

and the Christoffel symbols $\Gamma_{\alpha \beta}^{\gamma}$ defined by

$$
\Gamma_{\alpha \beta}^{\gamma}=\mathrm{e}^{\gamma} \cdot \mathrm{e}_{\alpha, \beta} .
$$

We then have

$$
\mathrm{e}_{\alpha, \beta}=\Gamma_{\alpha \beta}^{\gamma} \mathrm{e}_{\gamma}-\mathfrak{L}_{\alpha \beta} \boldsymbol{\infty}, \quad \mathrm{e}_{, \beta}^{\gamma}=-\Gamma_{\alpha \beta}^{\gamma} \mathrm{e}^{\alpha}-\mathfrak{L}_{\beta}^{\gamma} \boldsymbol{\gamma},
$$

where $\mathfrak{L}_{\alpha \beta}=\mathfrak{L}_{\mathbb{e}_{\alpha}} \cdot \mathbb{e}_{\beta}$ and $\mathfrak{L}_{\beta}^{\gamma}=\mathfrak{L}^{\gamma} \cdot \mathbb{e}_{\beta}$, and where $\mathfrak{L}$ is the curvature tensor of the reference configuration of the film, see (1). (We note in passing that the second fundamental form of $\mathcal{S}$ is given by $b_{\alpha \beta}=-\left\llcorner_{\alpha \beta}\right.$.) If $V$ is a finite-dimensional vector space and $f: \mathcal{S} \rightarrow V$ a class 2 mapping then

$$
\nabla f=f_{, \alpha} \otimes e^{\alpha}
$$

and consequently

$$
\nabla^{2} f=\left(f_{, \alpha} \otimes \Theta^{\alpha}\right)_{, \beta} \otimes \Theta^{\beta} .
$$

Differentiating the above product by the product rule and employing the formula for $\Theta_{, \beta}^{\alpha}$ given above we obtain

$$
\nabla^{2} f=f_{, \alpha \beta} \otimes \Theta^{\alpha} \otimes \Theta^{\beta}-f_{, \alpha} \otimes \Gamma_{\gamma \beta}^{\alpha} \Theta^{\gamma} \otimes e^{\beta}-\left\llcorner_{\beta}^{\alpha} f_{, \alpha} \otimes \infty \otimes \Theta^{\beta} .\right.
$$


It follows that

$$
\nabla^{2} f \circ(\bigoplus, P)=f_{, \alpha \beta} \otimes e^{\alpha} \otimes e^{\beta}-f_{, \alpha} \otimes \Gamma_{\gamma \beta}^{\alpha} e^{\gamma} \otimes e^{\beta} .
$$

If $V$ is a finite-dimensional vector space and $@: S \rightarrow \operatorname{Lin}\left(\mathrm{R}^{n}, V\right)$ a superficial map then we have the formula

$$
\operatorname{div} \mathbb{Q}=\mathbb{J}^{-1}\left(\mathbb{J} \mathbb{Q}^{\alpha}\right), \alpha,
$$

where $\mathbb{Q}^{\alpha}=\mathbb{Q}^{\alpha}$ and $\mathbb{J}=\left(\operatorname{det} \nabla \boldsymbol{\Phi}^{\mathrm{T}} \nabla \boldsymbol{\Phi}\right)^{1 / 2}$ is the jacobian of $\boldsymbol{\Phi}$. This formula coincides with the well known expression for the divergence based on covariant derivatives of tangential vector fields. However, with the divergence defined in (3), Formula (37) holds for an arbitrary superficial field $\mathbb{Q}: \mathcal{S} \rightarrow \operatorname{Lin}\left(\mathrm{R}^{n}, V\right)$, where $V$ is arbitrary finite-dimensional vector space with inner product, in particular also for second and third-order tensor fields, whereas (37) does not hold for divergence based on the covariant derivative of tensor fields of order $\geq 2$. See also (43) (below).

By (35) and (36), the first and second surface deformation gradients are determined by the components of the deformation function $y^{i}:=y \cdot \mathbf{e}^{i}$ as follows:

$$
\begin{gathered}
\mathbb{F}=\nabla \mathbb{y}=F_{\alpha}^{i} \mathbf{e}_{i} \otimes \Theta^{\alpha}, \\
\nabla^{2} y=F_{\alpha, \beta}^{i} \mathbf{e}_{i} \otimes \Theta^{\alpha} \otimes \Theta^{\beta}-F_{, \alpha}^{i}\left(\Gamma_{\gamma \beta}^{\alpha} \mathbf{e}_{i} \otimes \Theta^{\gamma} \otimes \Theta^{\beta}+\left\llcorner_{\beta}^{\alpha} \mathbf{e}_{i} \otimes \infty \otimes \Theta^{\beta}\right),\right.
\end{gathered}
$$

where

$$
F_{\alpha}^{i}=\mathbb{Y}_{, \alpha}^{i}
$$

It follows that

$$
\mathbb{G}=\nabla^{2} \mathbb{g} \circ(\mathbb{P}, \mathbb{P})=\left(\mathbb{F}_{\alpha, \beta}^{i}-\mathbb{F}_{, \gamma}^{i} \Gamma_{\alpha \beta}^{\gamma}\right) \mathbf{e}_{i} \otimes \Theta^{\alpha} \otimes \Theta^{\beta} .
$$

Let us express the energy as a function of $F_{\alpha}^{i}$ and $F_{\alpha, \beta}^{i}$, viz.,

$$
\hat{\mathbf{f}}(\mathbb{F}, \mathbb{G})=\overline{\mathfrak{f}}\left(\mathbb{F}_{\alpha}^{i}, F_{\alpha, \beta}^{i}\right),
$$

where $\mathbb{F}, \mathbb{G}$ and $\mathbb{F}_{\alpha}^{i}, F_{\alpha, \beta}^{i}$ are related by the formulas established above. This is the assumption employed in [Steigmann and Ogden 1999].

Proposition 7.1. We have

$$
\delta \mathrm{E}_{\mathrm{c}}(\mathbb{Y}, \mathbb{v})=-\int_{\mathcal{S}} \mathbb{J}^{-1}\left(\mathbb{J} \nabla_{i}^{\alpha}\right)_{, \alpha} \mathbb{v}^{i} d \mathcal{H}^{n-1}+\int_{\partial \mathcal{S}}\left(\mathbb{M}_{i}^{\alpha \beta} \mathbb{v}_{, \alpha}^{i} \varlimsup_{\beta}+\mathbb{\nabla}_{i}^{\alpha} \boldsymbol{\infty}_{\alpha} \mathbb{v}^{i}\right) d \mathcal{H}^{n-2}
$$

for each $\mathbf{v} \in C^{2}\left(\mathrm{cl} \mathcal{S}, \mathrm{R}^{n}\right)$, where

$$
\mathbb{\nabla}_{i}^{\alpha}=\partial_{\mathbb{F}_{\alpha}^{i}} \overline{\mathbf{f}}-\mathbb{J}^{-1}\left(\mathbb{J} \mathbb{M}_{i}^{\alpha, \beta}\right)_{, \beta}, \quad \mathbb{M}_{i}^{\alpha \beta}=\partial_{\mathbb{F}_{\alpha, \beta}^{i}} \overline{\mathfrak{f}} ;
$$

furthermore $\nabla_{i}^{\alpha}$ and $\mathbb{M}_{i}^{\alpha \beta}$ are the components of the tensors $\nabla$ and $\mathbb{M}$, satisfying

$$
\nabla=\nabla_{i}^{\alpha} \mathbf{e}^{i} \otimes \Theta_{\alpha}, \quad \mathbb{M}=\mathbb{M}_{i}^{\alpha \beta} \mathbf{e}^{i} \otimes \Theta_{\alpha} \otimes \Theta_{\beta} .
$$


Up to a change of notation, (39) coincides with Equation (4.8) of [Steigmann and Ogden 1999].

Proof. If $\nabla_{i}^{\alpha}$ and $\mathbb{M}_{i}^{\alpha \beta}$ are the components of $\nabla$ and $\mathbb{M}$, we see that (10) reduces to (39). Thus it remains to prove (40). Let the components $\mathbb{S}_{i}^{\alpha}$ of $\mathbb{S}$ be defined by

$$
\mathrm{S}=\mathbb{S}_{i}^{\alpha} \mathbf{e}^{i} \otimes \mathbb{e}_{\alpha} .
$$

The components of the partial derivatives $\hat{\mathbb{f}}$ and the partial derivatives of $\bar{f}$ are related as follows:

$$
\left.\mathbb{S}_{i}^{\alpha} \equiv \partial_{\mathbb{F}} \hat{\mathbf{f}}\right|_{i} ^{\alpha}=\partial_{\mathbb{F}_{\alpha}^{i}} \overline{\mathbf{f}}+\partial_{\mathbb{F}_{\gamma, \beta}^{i}} \overline{\mathfrak{f}} \Gamma_{\gamma \beta}^{\alpha},\left.\quad \mathbb{M}_{i}^{\alpha \beta} \equiv \partial_{\mathbb{G}} \hat{\mathbf{f}}\right|_{i} ^{\alpha \beta}=\partial_{\mathbb{F}_{\alpha, \beta}^{i}, \overline{\mathbf{f}}},
$$

where we have used (38). Equation $(40)_{2}$ then follows immediately from $(41)_{2}$. To prove $(40)_{1}$, we note that by (37), we have

$$
\begin{aligned}
(\operatorname{div} \mathbb{M})_{i} & =\mathbb{J}^{-1}\left(\mathbb{J M}_{i}^{\alpha \beta} \mathbb{e}_{\alpha}\right)_{, \beta} \\
& =\mathbb{J}^{-1}\left(\mathbb{J} \mathbb{M}_{i}^{\alpha \beta}\right)_{, \beta} \Theta_{\alpha}+\mathbb{M}_{i}^{\alpha \beta} \Theta_{\alpha, \beta} \\
& =\mathbb{J}^{-1}\left(\mathbb{J}_{i}^{\alpha \beta}\right)_{, \beta} \Theta_{\alpha}+\mathbb{M}_{i}^{\alpha \beta}\left(\Gamma_{\alpha \beta}^{\gamma} \mathbb{e}_{\gamma}-\mathbb{L}_{\alpha \beta} \varpi\right),
\end{aligned}
$$

where $\left.(\operatorname{div} \mathbb{N})_{i}=(\operatorname{div} \mathbb{M})\right)^{\mathrm{T}} \mathbf{e}_{i}$. Thus

$$
((\text { div } \mathbb{M}) \mathbb{P})_{i}=\mathbb{J}^{-1}\left(\mathbb{J} \mathbb{M}_{i}^{\alpha \beta}\right)_{, \beta} \mathbb{e}_{\alpha}+\mathbb{\mathbb { N } _ { i } ^ { \alpha \beta }} \Gamma_{\alpha \beta}^{\gamma} \mathbb{e}_{\gamma},
$$

where $((\operatorname{div} \mathbb{M}) \mathbb{P})_{i}=((\operatorname{div} \mathbb{M}) \mathbb{P})^{\mathrm{T}} \mathbf{e}_{i}$. Combining (42) with $(41)_{1}$, we obtain $(40)_{1}$.

Next, let us express the energy as a function $\overline{\bar{f}}$ of $\mathbb{F}_{\alpha}^{i}$ and $\mathbb{K}_{\alpha \beta}:=\mathbb{K}\left(\Theta_{\alpha}, e_{\beta}\right)$. We have

$$
\mathbb{K}_{\alpha \beta}=\overline{\mathfrak{N}}_{i} \mathbb{G}_{\alpha \beta}^{i},
$$

where

$$
\mathbb{G}_{\alpha \beta}^{i}=\mathbf{e}^{i} \cdot \mathbb{G}\left(\mathbb{e}_{\alpha}, \mathbb{e}_{\beta}\right)=\mathbb{F}_{\alpha, \beta}^{i}-\mathbb{F}_{, \gamma}^{i} \Gamma_{\alpha \beta}^{\gamma},
$$

where we have used (38). Using the relation $\bar{\varpi}_{i} F_{,}^{i}, \gamma=0$ we obtain

$$
\mathfrak{K}_{\alpha \beta}=\overline{\mathfrak{N}}_{i} \mathbb{F}_{\alpha, \beta}^{i} .
$$

The function $\overline{\overline{\mathrm{f}}}$ satisfies the relation

$$
\overline{\overline{\mathrm{f}}}\left(\mathbb{F}_{\alpha}^{i}, \mathbb{K}_{\alpha \beta}\right)=\overline{\mathbf{f}}\left(\mathbb{F}_{\alpha}^{i}, \mathbb{F}_{\alpha, \beta}^{i}\right) .
$$

Proposition 7.2. In terms of the partial derivatives of $\overline{\overline{\mathbf{f}}}$ we have

$$
\nabla_{i}^{\alpha}=\partial_{\mathbb{F}_{\alpha} i} \overline{\overline{\mathbb{f}}}-\mathbb{J}^{-1}\left(\mathbb{F}^{-1}\right)_{j}^{\alpha}\left(\mathbb{J} \overline{\mathfrak{w}}_{i} \mathbb{F}_{\beta}^{j} \partial_{\mathbb{K}_{\beta \gamma}} \overline{\overline{\mathbb{f}}}\right)_{, \gamma},
$$


where the components $\left(\mathbb{F}^{-1}\right)_{j}^{\alpha}$ are defined via the identification

$$
F^{-1}=\left(F^{-1}\right)_{j}^{\alpha} \Theta_{\alpha} \otimes \mathbf{e}^{j},
$$

where $F^{-1}$ is the pseudoinverse of $F^{5}$.

Proof. This follows immediately from Proposition 5.4.

We note that the parametrization $\boldsymbol{\Phi}$ of the referential surface $\mathcal{S}$ which introduces a coordinate system $\theta^{1}, \ldots, \theta^{n-1}$ on $\mathcal{S}$ gives, via the composition with the deformation $\mathrm{y}$ a parametrization $\overline{\boldsymbol{\Phi}}:=$ y $\circ \boldsymbol{\Phi}$ of the deformed surface $\overline{\mathcal{S}}$ which introduces the coordinate system $\theta^{1}, \ldots, \theta^{n-1}$ on $\overline{\mathcal{S}}$. If $m$ is a function defined on $\overline{\mathcal{S}}$ with values in a finite-dimensional vector space, we use the subscript comma followed by the index $\alpha$ to denote the derivative of $m \circ \overline{\boldsymbol{\Phi}}$ with respect to $\theta^{\alpha}$. The coordinate vectors $\overline{\mathrm{e}}^{\alpha}$ corresponding to the coordinate system $\theta^{1}, \ldots, \theta^{n-1}$ on $\overline{\mathcal{S}}$ are given by $\overline{\mathrm{e}}^{\alpha}=F \mathrm{e}^{\alpha}$ and the dual vectors are $\overline{\mathrm{e}}_{\alpha}=F^{-\mathrm{T}} \Theta_{\alpha}$. We denote by $\bar{\Gamma}_{\alpha \beta}^{\gamma}$ the Christoffel symbols corresponding to the coordinate system $\theta^{1}, \ldots, \theta^{n-1}$ on $\overline{\mathcal{S}}$, given by

$$
\bar{\Gamma}_{\alpha \beta}^{\gamma}=\overline{\mathrm{e}}^{\gamma} \cdot \overline{\mathrm{e}}_{\alpha, \beta} .
$$

We denote by a vertical bar followed by an index $\alpha$ the covariant differentiation on $\overline{\mathcal{S}}$ using the Christoffel symbols $\bar{\Gamma}_{\alpha \beta}^{\gamma}$, i.e., if $\overline{\mathrm{v}}=\overline{\mathrm{v}}^{\alpha} \overline{\mathrm{e}}_{\alpha}$ and $\overline{\mathbb{A}}=\overline{\mathbb{A}}^{\alpha \beta} \overline{\mathrm{e}}_{\alpha} \otimes \overline{\mathrm{e}}_{\beta}$ is a tangential vector and superficial tensor defined on $\overline{\mathcal{S}}$ then

$$
\begin{gathered}
\left.\overline{\mathbf{v}}^{\alpha}\right|_{\beta}=\overline{\mathbb{v}}_{, \beta}^{\alpha}-\bar{\Gamma}_{\beta \gamma}^{\alpha} \overline{\mathbf{v}}^{\gamma}, \\
\left.\overline{\boldsymbol{A}}^{\alpha \beta}\right|_{\gamma}=\overline{\mathbf{A}}_{, \gamma}^{\alpha \beta}-\bar{\Gamma}_{\gamma \delta}^{\alpha} \overline{\boldsymbol{A}}^{\delta \beta}-\bar{\Gamma}_{\gamma \delta}^{\beta} \overline{\mathbf{A}}^{\alpha \delta} .
\end{gathered}
$$

We shall also employ the divergences based on the covariant differentiation, i.e., the objects $\left.\overline{\mathbf{v}}^{\alpha}\right|_{\alpha}$ and $\left.\overline{\mathbb{A}}^{\alpha \beta}\right|_{\beta}$. It is easy to see that the superficial derivative is related to the just mentioned divergences by

$$
\operatorname{div} \overline{\mathbb{A}}=\left.\overline{\mathbf{v}}^{\alpha}\right|_{\alpha}, \quad \bar{P} \operatorname{div} \overline{\mathbb{A}}=\left.\overline{\mathbb{A}}^{\alpha \beta}\right|_{\beta} \bar{\Theta}_{\alpha} .
$$

For the subsequent discussion we define the superficial right Cauchy-Green tensor components $\mathbb{C}_{\alpha \beta}=\mathbb{F}_{\alpha}^{i} \mathbb{F}_{\beta}^{i}$. Furthermore, assume that the stored energy $\tilde{\mathrm{f}}$ is objective and let us express the energy as a function $\hat{\hat{f}}$ of $\mathbb{C}_{\alpha \beta}$ and $\mathbb{K}_{\alpha \beta}$, i.e.,

$$
\hat{\hat{\mathfrak{f}}}\left(\mathbb{C}_{\alpha \beta}, \mathbb{K}_{\alpha \beta}\right)=\tilde{\mathfrak{f}}(\mathbb{F}, \mathbb{K})=\overline{\overline{\mathbf{f}}}\left(\mathbb{F}_{\alpha}^{i}, \mathbb{K}_{\alpha \beta}\right) .
$$

We note that this is possible by the objectivity.

Proposition 7.3. Assume that the stored energy $\tilde{\mathbf{f}}$ is objective and denote by $\overline{\mathbb{N}}^{\alpha \beta}$ and $\overline{\mathbb{N}}^{\alpha \beta}$ the components of $\overline{\mathbb{N}}$ and $\overline{\mathbb{M}}$ identified by

$$
\overline{\mathbb{N}}=\overline{\mathbb{N}}^{\alpha \beta} \bar{\Theta}_{\alpha} \otimes \bar{\Theta}_{\beta}, \quad \overline{\mathbb{M}}=\overline{\mathbb{M}}^{\alpha \beta}{\overline{\Theta_{\alpha}}}_{\alpha} \otimes \bar{\Theta}_{\beta} .
$$


In terms of these components, Equations (26) read

$$
\left.\begin{array}{c}
\left.\left(\overline{\mathbb{N}}^{\alpha \beta}-\overline{\mathbb{L}}_{\gamma}^{\alpha} \overline{\mathbb{M}}^{\gamma \beta}\right)\right|_{\beta}-\left.\overline{\mathbb{L}}_{\gamma}^{\alpha} \overline{\mathbb{M}}^{\gamma \beta}\right|_{\beta}+\mathfrak{j}^{-1} \mathbb{P}^{\alpha}=0, \\
\left.\overline{\mathbb{N}}^{\alpha \beta}\right|_{\alpha \beta}+\overline{\mathbb{L}}_{\alpha \beta} \overline{\mathbb{N}}^{\alpha \beta}-\overline{\mathbb{L}}_{\alpha \gamma} \overline{\mathbb{L}}_{\beta}^{\gamma} \cdot \overline{\mathbb{N}}^{\alpha \beta}-\mathfrak{j}^{-1} \mathbb{P}^{\perp}=0,
\end{array}\right\}
$$

where

$$
\mathbb{P}^{\|}=\mathbb{P}^{\alpha} \bar{\Theta}_{\alpha} .
$$

If $n=3$, the system of boundary conditions $(24)_{1},(27)_{1}$, and (28) is equivalent to

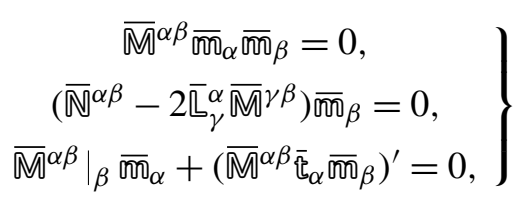

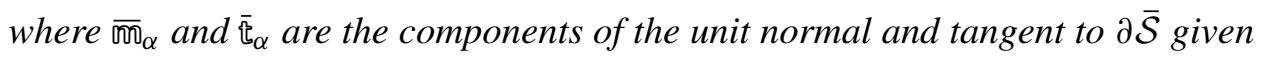
by

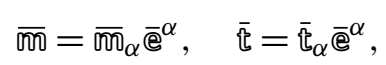

and the superscript' denotes the derivative with respect to the arc length parameter on $\partial \overline{\mathcal{S}}$. One has

$$
\overline{\mathbb{N}}^{\alpha \beta}=\mathfrak{j}^{-1} \partial_{\mathbb{C}_{\alpha \beta}} \hat{\hat{\mathbf{f}}}, \quad \overline{\mathbb{N}}^{\alpha \beta}=\mathfrak{j}^{-1} \partial_{\mathbb{K}_{\alpha \beta}} \hat{\hat{\mathbf{f}}} .
$$

Apart from differences in notation, Equations (44) coincide with Equations (4.37) of [Steigmann and Ogden 1999]. They also coincide with the first and second of equations (9.47) of [Naghdi 1971] when the latter are specialized to the case of equilibrium of a shell, and with the equations in Theorem 7.1-3 of [Ciarlet 2000]. Proof. Equations (44) follow from (26) and the identities (43).

To prove (46), we note that differentiating the relation

$$
\tilde{\mathbf{f}}\left(\mathbb{F}_{\alpha}^{i} \mathbf{e}_{i} \otimes e^{\alpha}, \mathbb{K}_{\alpha \beta} \Theta^{\alpha} \otimes \Theta^{\beta}\right)=\overline{\overline{\mathbf{f}}}\left(\mathbb{F}_{\alpha}^{i}, \mathfrak{K}_{\alpha \beta}\right)
$$

one obtains

$$
\partial_{\mathbb{F}} \tilde{\mathbf{f}}=\partial_{F_{\alpha} i} \overline{\overline{\mathbf{f}}} \mathbf{e}^{i} \otimes \Theta_{\alpha}, \quad \partial_{\mathbb{K}} \tilde{\mathbf{f}}=\partial_{\mathbb{K}_{\alpha \beta}} \overline{\overline{\mathbf{f}}} \Theta_{\alpha} \otimes \Theta_{\beta} .
$$

From (47) ${ }_{1}$ follows that

$$
\overline{\mathbb{N}}_{i j}=\mathfrak{j}^{-1} \partial_{\mathbb{F}_{\alpha}^{i}} \overline{\overline{\mathrm{f}}} \vec{F}_{\alpha}^{j},
$$

where $\overline{\mathbb{N}}_{i j}$ are the components of $\overline{\mathbb{N}}$ is the orthonormal basis $\mathbf{e}^{i} \equiv \mathbf{e}_{i}$. The components of $\overline{\mathbb{N}}$ in the basis $\bar{\Theta}_{\alpha}=F_{\alpha}^{i} \mathbf{e}_{i}$ are then related by $\overline{\mathbb{N}}_{i j}=\overline{\mathbb{N}}^{\alpha} \vec{F}_{\alpha}^{i} \mathbb{F}_{\beta}^{j}$, which gives

$$
\overline{\mathbb{N}}^{\alpha \beta}=\overline{\mathbb{N}}_{i j}\left(\mathbb{F}^{-1}\right)_{i}^{\alpha}\left(\mathbb{F}^{-1}\right)_{j}^{\beta}=\mathfrak{j}^{-1}\left(\mathbb{F}^{-1}\right)_{i}^{\alpha} \partial_{\mathbb{F}_{\beta}^{i}} \overline{\overline{\mathfrak{f}}} .
$$

Likewise, from $(47)_{2}$ follows that

$$
\overline{\mathbb{M}}_{i j}=\mathfrak{j}^{-1} \vec{F}_{\alpha}^{i} \mathbb{F}_{\beta}^{j} \partial_{\mathbb{K}_{\alpha \beta}} \overline{\overline{\mathbf{f}}}
$$


where $\overline{\mathbb{M}}_{i j}$ are the components of $\overline{\mathbb{M}}$ in the basis $\mathbf{e}^{i} \equiv \mathbf{e}_{i}$. It follows as above that the components $\overline{\mathbb{N}}^{\alpha \beta}$ in the basis $\overline{\mathrm{e}}_{\alpha}$ are

$$
\overline{\mathbb{M}}^{\alpha \beta}=\overline{\mathbb{M}}_{i j}\left(\mathbb{F}^{-1}\right)_{i}^{\alpha}\left(\mathbb{F}^{-1}\right)_{j}^{\beta}=\mathfrak{j}^{-1} \partial_{\mathbb{K}_{\alpha \beta}} \overline{\overline{\mathbb{f}}} .
$$

Differentiating the relation

$$
\hat{\hat{f}}\left(\mathbb{F}_{\alpha}^{i} \mathbb{F}_{\beta}^{i}, \mathbb{K}_{\alpha \beta}\right)=\overline{\overline{\mathfrak{f}}}\left(\mathbb{F}_{\alpha}^{i}, \mathbb{K}_{\alpha \beta}\right)
$$

we obtain

$$
\partial_{\mathbb{C}_{\alpha \beta}} \hat{\hat{f}}_{F_{\beta}^{i}}^{i}=\partial_{F_{\alpha}^{i}} \overline{\overline{\mathbf{f}}}, \quad \partial_{\mathbb{K}_{\alpha \beta}} \hat{\hat{\mathbf{f}}}=\partial_{\mathbb{K}_{\alpha \beta}} \overline{\overline{\mathrm{f}}} .
$$

A combination of (48) with (50) 1 provides $(46)_{1}$ and a combination of (49) with $(50)_{2}$ provides $(46)_{2}$.

The equivalence of the system $(24)_{1},(27)_{1}$, and (28) with (45) is proved similarly.

\section{References}

[Ciarlet 2000] P. G. Ciarlet, Mathematical elasticity, III: Theory of shells, Studies in Mathematics and its Applications 29, North-Holland, Amsterdam, 2000.

[Cohen and De Silva 1966] H. Cohen and C. N. De Silva, "Nonlinear theory of elastic surfaces", J. Mathematical Phys. 7 (1966), 246-253.

[Delfour and Zolésio 1997] M. C. Delfour and J.-P. Zolésio, "Differential equations for linear shells: comparison between intrinsic and classical models", pp. 41-124 in Advances in mathematical sciences: CRM's 25 years (Montreal, 1994), edited by L. Vinet, CRM Proc. Lecture Notes 11, Amer. Math. Soc., Providence, RI, 1997.

[Favata and Podio-Guidugli 2011] A. Favata and P. Podio-Guidugli, "A new CNT-oriented shell theory”, Eur. J. Mech. A Solids 35 (2011), 75-96.

[Federer 1969] H. Federer, Geometric measure theory, Grundlehren math. Wiss. 153, Springer, New York, 1969.

[Fried and Todres 2005] E. Fried and R. E. Todres, "Mind the gap: the shape of the free surface of a rubber-like material in proximity to a rigid contactor", J. Elasticity 80:1-3 (2005), 97-151.

[Gurtin 2000] M. E. Gurtin, Configurational forces as basic concepts of continuum physics, Applied Mathematical Sciences 137, Springer, New York, 2000.

[Gurtin and Murdoch 1975] M. E. Gurtin and A. I. Murdoch, "A continuum theory of elastic material surfaces", Arch. Rational Mech. Anal. 57 (1975), 291-323.

[Hilgers and Pipkin 1992] M. G. Hilgers and A. C. Pipkin, "Elastic sheets with bending stiffness", Quart. J. Mech. Appl. Math. 45:1 (1992), 57-75.

[Marsden and Hughes 1983] J. E. Marsden and T. J. R. Hughes, Mathematical foundations of elasticity, Prentice-Hall, Englewood Cliffs, NJ, 1983.

[Murdoch and Cohen 1979/80] A. I. Murdoch and H. Cohen, "Symmetry considerations for material surfaces", Arch. Rational Mech. Anal. 72:1 (1979/80), 61-98.

[Naghdi 1971] P. M. Naghdi, The theory of shells and plates, edited by C. Truesdell, Handbuch der Physik VIa/2, Springer, Berlin, 1971. 
[Pietraszkiewicz 1989] W. Pietraszkiewicz, "Geometrically nonlinear theories of thin elastic shells", Adv. in Mech. 12:1 (1989), 51-130.

[Podio-Guidugli 1988] P. Podio-Guidugli, "A variational approach to live loadings in finite elasticity", J. Elasticity 19:1 (1988), 25-36.

[Podio-Guidugli and Vergara-Caffarelli 1990] P. Podio-Guidugli and G. Vergara-Caffarelli, "Surface interaction potentials in elasticity", Arch. Rational Mech. Anal. 109:4 (1990), 343-383.

[Sanders 1963] J. L. Sanders, Jr., "Nonlinear theories for thin shells", Quart. Appl. Math. 21 (1963), 21-36.

[Šilhavý 2011] M. Šilhavý, "Equilibrium of phases with interfacial energy: a variational approach", J. Elasticity 105:1-2 (2011), 271-303.

[Steigmann and Li 1995] D. J. Steigmann and D. Li, "Remarks on a theory of elasticity for fluid films", Proc. Royal Soc. London 449 (1995), 223-231.

[Steigmann and Ogden 1997a] D. J. Steigmann and R. W. Ogden, "Plane deformations of elastic solids with intrinsic boundary elasticity", Proc. Roy. Soc. London Ser. A 453:1959 (1997), 853877.

[Steigmann and Ogden 1997b] D. J. Steigmann and R. W. Ogden, "A necessary condition for energyminimizing plane deformations of elastic solids with intrinsic boundary elasticity", Math. Mech. Solids 2:1 (1997), 3-16.

[Steigmann and Ogden 1999] D. J. Steigmann and R. W. Ogden, "Elastic surface-substrate interactions”, R. Soc. Lond. Proc. Ser. A Math. Phys. Eng. Sci. 455:1982 (1999), 437-474.

[Steinmann 2008] P. Steinmann, "On boundary potential energies in deformational and configurational mechanics", J. Mech. Phys. Solids 56:3 (2008), 772-800.

Received 29 Feb 2012. Revised 17 Sep 2012. Accepted 20 Oct 2012.

MIROSLAV ŠILHAVÝ: silhavy@math.cas.cz

Institute of Mathematics, Academy of Sciences of the Czech Republic, Žitná 25, 11567 Prague 1, Czech Republic 


\title{
A SUFFICIENT CONDITION FOR A DISCRETE SPECTRUM OF THE KIRCHHOFF PLATE WITH AN INFINITE PEAK
}

\author{
Fedor L. Bakharev, Sergey A. Nazarov and Guido H. Sweers \\ Sufficient conditions for a discrete spectrum of the biharmonic equation in a two- \\ dimensional peak-shaped domain are established. Different boundary conditions \\ from Kirchhoff's plate theory are imposed on the boundary and the results de- \\ pend both on the type of boundary conditions and the sharpness exponent of the \\ peak.
}

\section{Motivation}

Elliptic boundary value problems on domains which have a Lipschitz boundary and a compact closure, in particular when they generate positive self-adjoint operators, have fully discrete spectra. However, if the domain loses the Lipschitz property or compactness, other situations may occur. It is well-known that for the Dirichlet case boundedness is sufficient but not necessary for having discrete spectrum. See the famous paper [Rellich 1948] or the more recent [Rozenbljum 1972; van den Berg 1984]. On the other hand, for the Neumann problem of the Laplace operator there exist numerous examples of bounded domains such that the spectrum gets a nonempty continuous component (see e.g. [Courant and Hilbert 1953; Maz'ya and Poborchii 2006; 1997; Simon 1992; Hempel et al. 1991]).

The literature on the spectra for the Laplace operator with various boundary conditions on special domains is focused on domains that have a cusp, a finite or infinite peak or horn [van den Berg 1984; Hempel et al. 1991; Jakšić et al. 1992; Davies and Simon 1992; Jakšić 1993; Ivrii 1999; Boyarchenko and Levendorskii 2000; van den Berg and Lianantonakis 2001; Kovař́k 2011] or even a rolled horn [Simon 1992].

The criteria in [Adams and Fournier 2003] and [Evans and Harris 1987] for the embedding $H^{1}(\Omega) \subset L_{2}(\Omega)$ to be compact show that the Neumann-Laplace

This work is supported by the Russian Foundation for Basic Research (project 12-01-00348). Bakharev is also supported by the Chebyshev Laboratory (Department of Mathematics and Mechanics, Saint Petersburg State University) under the grant 11.G34.31.0026 of the Government of the Russian Federation and grant 6.38.64.2012 of Saint Petersburg State University.

MSC2010: 35P05, 47A10, 74K20.

Keywords: Kirchhoff plate, cusp, peak, discrete and continuous spectra. 
problem on a domain $\Omega$ with the infinite peak

$$
\Pi_{R}=\left\{x=\left(x_{1}, x_{2}\right) \in \mathbb{R}^{2}: x_{1}>R,-H\left(x_{1}\right)<x_{2}<H\left(x_{1}\right)\right\},
$$

where the function $H>0$ is smooth and monotone decreasing, has discrete spectrum if and only if

$$
\lim _{y \rightarrow+\infty} \int_{y}^{+\infty} \frac{H(\eta)}{H(y)} d \eta=0 \quad\left(\Longleftrightarrow \lim _{y \rightarrow+\infty} \frac{H(y+\epsilon)}{H(y)}=0 \text { for any } \epsilon>0\right) .
$$

The function $H$ is assumed to have a first derivative that tends to zero and a bounded second derivative. It will be convenient to use the notation $\Upsilon(y)=(-H(y), H(y))$. Here is an image of such a domain:

The simplest boundary irregularity violating the Lipschitz condition is just the (finite) peak

$$
\varpi_{R}=\left\{x: 0<x_{1}<R,-h\left(x_{1}\right)<x_{2}<h\left(x_{1}\right)\right\},
$$

where $h\left(x_{1}\right)=h_{0} x_{1}^{1+\alpha}, h_{0}>0$ and $\alpha>0$. Nevertheless, the spectrum of the Neumann problem in the domain with this peak stays discrete. See Remark 5.1.

A criterion ([Nazarov 2009]) for having essential spectrum in the Neumann problem for elliptic systems of second order differential equations with a polynomial property is derived in [Nazarov 1999]. In particular it shows that the continuous spectrum of an elastic body with $\alpha \geq 1$ for the peak (3) is nonempty (see [Nazarov 2008; Bakharev and Nazarov 2009]). This phenomenon of generating wave processes in a finite volume, is known experimentally and used in the engineering practice to construct wave dampers, "black holes", for elastic oscillations (see [Mironov 1988; Krylov and Tilman 2004], etc.).

In this paper we study the spectra of boundary value problems for the Kirchhoff model of a thin elastic plate described by the biharmonic operator $\Delta^{2}$. The boundary conditions that we consider model the three mechanically most reasonable cases, namely where the lateral sides of the peak are supplied with one of the following three types of the boundary conditions: clamped edge (Dirichlet), traction-free edge (Neumann) and hinged edge (Mixed). In all these cases the spectrum of the problem in a bounded domain with the peak as in (3) is discrete. We derive sufficient conditions for the spectrum to be discrete for the boundary value problem on an unbounded domain with a peak as in (1).

If a sufficient number of Dirichlet conditions are imposed on the lateral sides of the peak (the cases $\boldsymbol{D}-\boldsymbol{N}, \boldsymbol{M}-\boldsymbol{M}, \boldsymbol{D}-\boldsymbol{M}$, and $\boldsymbol{D}-\boldsymbol{D}$; see formulas (5)-(7) and 
(11), (12)), then the proof that the spectrum is discrete becomes rather simple (Theorem 5). Indeed, it suffices to apply the weighted Friedrich's inequality (13) and to take into account the decay of the quantity $H(y)$ as $y \rightarrow+\infty$.

Our main interest concerns the cases $\boldsymbol{M}-\boldsymbol{N}$ and $\boldsymbol{N}-\boldsymbol{N}$. By applying weighted inequalities of Hardy type (Lemmas 7 and 8) we obtain a sufficient condition for the case $\mathbf{N}-\boldsymbol{N}$ to have discrete spectrum (Proposition 4). Indeed, as shown in [Adams and Fournier 2003], the second condition in (2) implies a criterion for the compact embedding $H^{m}(\Omega) \subset L_{2}(\Omega)$ for all $m$ (we just need $m=2$ ). One can also use that approach (Theorem 12) for the case $\boldsymbol{M}-\boldsymbol{N}$. This approach differs from the one used in [Adams and Fournier 2003; Evans and Harris 1987]. The different argument allows to obtain a condition for having discrete spectrum if one of the peak's edges is traction-free and the other one is hinged (the case $\boldsymbol{N}-\boldsymbol{M}$; see Theorem 13).

The obtained results essentially differ from each other: under the conditions (11) and also under (12) any decay of $H$ is enough, the case $\boldsymbol{M}-\boldsymbol{N}$ needs a power decay rate with the exponent $\alpha>1$, while the case $N-N$ needs a superexponential decay rate. See Remark 12.1 and 13.1.

\section{The Kirchhoff plate model}

Assumption 1. Let $\Omega$ be a domain in the plane $\mathbb{R}^{2}$ with a smooth (of class $C^{\infty}$ ) boundary $\Gamma$ such that, for some $R>0$ and some monotone decreasing function $H:[R, \infty) \rightarrow \mathbb{R}^{+}$with $\lim _{t \rightarrow \infty} H(t)=0$,

(1) $\left\{\left(x_{1}, x_{2}\right) \in \Omega ; x_{1}>R\right\}=\left\{\left(x_{1}, x_{2}\right) ; x_{1}>R\right.$ and $\left.\left|x_{2}\right|<H\left(x_{1}\right)\right\}$ and

(2) $\left\{\left(x_{1}, x_{2}\right) \in \Omega ; x_{1}<R\right\}$ is bounded.

We regard $\Omega$ as the projection of a thin isotropic homogeneous plate and apply the Kirchhoff theory (see [Mikhlin 1970, §30], [Nazarov 2002, Chapter 7], and so on). So we arrive at the fourth-order differential equation

$$
\Delta^{2} u(x)=\lambda u(x), \quad x \in \Omega,
$$

which describes transverse oscillations of the plate. Here, $u(x)$ is the plate deflection, and $\lambda$ a spectral parameter proportional to the square of the oscillation frequency.

The following sets of boundary conditions have a clear physical interpretation (see [Mikhlin 1970, §30], [Gazzola et al. 2010, §1.1], and so on):

(D): Dirichlet for a clamped edge:

$$
u(x)=\partial_{n} u(x)=0, \quad x \in \Gamma_{D} .
$$


$(N)$ : Neumann for a traction-free edge:

$$
\left\{\begin{array}{c}
\partial_{n} \Delta u(x)-(1-v)\left(\partial_{s} \varkappa(x) \partial_{s} u(x)-\partial_{s}^{2} \partial_{n} u(x)\right)=0, \\
\Delta u(x)-(1-v)\left(\partial_{s}^{2} u(x)+\varkappa(x) \partial_{n} u(x)\right)=0,
\end{array} \quad x \in \Gamma_{N} .\right.
$$

$(M)$ : Mixed for a hinged edge:

$$
u=\Delta u(x)-(1-v) \varkappa(x) \partial_{n} u(x)=0, \quad x \in \Gamma_{M} .
$$

Here, $\partial_{n}$ and $\partial_{s}$ stand for the normal and tangential derivatives, $\varkappa(x)$ is the signed curvature of the contour $\Gamma$ at the point $x \in \Gamma$ positive for convex boundary parts, and $v \in[0,1 / 2)$ is the Poisson ratio. Finally, $\Gamma_{D}, \Gamma_{N}$, and $\Gamma_{M}$ are the unions of finite families of open curves and $\Gamma=\overline{\Gamma_{D}} \cup \overline{\Gamma_{N}} \cup \overline{\Gamma_{M}}$, two of which may be empty. In what follows it is convenient to use the notation $y=x_{1}$ and $z=x_{2}$.

The general properties of the spectra depend on which of the boundary conditions (5)-(7) are imposed on the upper (+) and lower (-) sides,

$$
\Sigma_{R}^{ \pm}=\{x: y>R, z= \pm H(y)\}
$$

of the peak. Let us give a precise statement. We define a symmetric bilinear form on $H^{2}(\Omega)$ by

$$
a(u, u)=\int_{\Omega}\left(\left|\frac{\partial^{2} u}{\partial x_{1}^{2}}\right|^{2}+\left|\frac{\partial^{2} u}{i} \partial x_{2}^{2}\right|^{2}+2(1-v)\left|\frac{\partial^{2} u}{\partial x_{1} \partial x_{2}}\right|^{2}+2 v \frac{\partial^{2} u}{\partial x_{1}^{2}} \frac{\partial^{2} u}{\partial x_{2}^{2}}\right) d x
$$

and $a(u, v)=\frac{1}{4} a(u+v, u+v)-\frac{1}{4} a(u-v, u-v)$. Then $\frac{1}{2} a(u, u)$ is the elastic energy stored in the plate. Since one directly verifies that

$$
a(u, u) \geq(1-v) \sum_{j, k=1}^{2} \int_{\Omega}\left|\frac{\partial^{2} u}{\partial x_{j} \partial x_{k}}\right|^{2} d x,
$$

the bilinear form $a(\cdot, \cdot)$ is nonnegative.

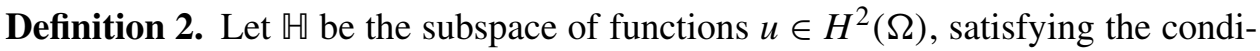
tions (5) on $\Gamma_{D}$ and $u=0$ on $\Gamma_{M}$ in the sense of traces.

By [Birman and Solomyak 1980, §10.1] $\mathbb{W}$ is a Hilbert space with scalar product $a(\cdot, \cdot)+(\cdot, \cdot)_{\Omega}$, where $(\cdot, \cdot)_{\Omega}$ is the standard scalar product in the Lebesgue space $L_{2}(\Omega)$. Moreover, Theorem 2 of [Birman and Solomyak 1980, §10.1] implies that there exists a unique (unbounded) self-adjoint operator $A: D(A) \subset$ $L_{2}(\Omega) \rightarrow L_{2}(\Omega)$ with $D(A) \subset D\left(A^{1 / 2}\right)=\mathbb{H}$ such that

$$
(A u, v)_{\Omega}=a(u, v) \text { for all } v \in \mathbb{H} .
$$


Note that the eventually remaining boundary conditions in (5)-(7) appear in $D(A) \subset$ $H^{4}(\Omega)$ as intrinsic natural boundary conditions from (9)-(8), see again [Mikhlin 1970, §30], [Gazzola et al. 2010, §1.1] etc.

Definition 3. By the spectrum for (4)-(7) we will mean $\sigma(A)$ with $A$ defined in (10).

Since $a$ is nonnegative, the spectrum $\sigma(A)$ belongs to $[0, \infty)$.

As a direct consequence of known results we may state the following.

Proposition 4. If (2) holds, then the spectrum of the Equation (4), with either of the above boundary condition on the sides of the peak, is discrete.

Proof. By [Birman and Solomyak 1980, $\S 10.1$ Theorem 5] the spectrum is discrete if and only if the embedding $\mathbb{U} \hookrightarrow L_{2}(\Omega)$ is compact. By [Adams and Fournier 2003; Evans and Harris 1987] one knows that $H^{2}(\Omega) \hookrightarrow L_{2}(\Omega)$ is compact whenever (2) holds true and $\mathbb{H} \subset H^{2}(\Omega)$.

\section{Simple cases: $D-N, M-M, D-M$ and $D-D$,}

Theorem 5. Suppose $\Omega$ is as in Assumption 1 and suppose that the boundary conditions for problem (4), as given in (5)-(7) contain one of the cases (11) or (12). Then the spectrum is discrete.

Remark 5.1. By a similar reasoning, we may conclude that in the bounded domain $\omega$, with the peak as in (3), Equation (4) has discrete spectrum for any set of conditions (5)-(7) on the arc $\partial \omega \backslash 0$. This fact follows from the inequality (see [Nazarov and Taskinen 2008]):

$$
\left\||x|^{-1} u ; L_{2}(\omega)\right\|^{2} \leq c\left(\left\|\nabla u ; L_{2}(\omega)\right\|^{2}+\left\|u ; L_{2}\left(\omega \backslash \varpi_{R}\right)\right\|^{2}\right) .
$$

Proof. By assumption the boundary conditions provide at least one of the following two groups of relations:

$$
\begin{array}{ll}
u=0 & \text { on } \Sigma_{R}^{+} \cup \Sigma_{R}^{-} ; \\
u=\partial_{n} u=0 & \text { on } \Sigma_{R}^{+} \text {or on } \Sigma_{R}^{-} .
\end{array}
$$

In both cases (11) and (12) the following version of Friedrich's inequality is valid:

$$
\int_{\Upsilon(y)}\left|\frac{\partial^{2} u}{\partial z^{2}}(y, z)\right|^{2} d z \geq \frac{c}{H(y)^{4}} \int_{\Upsilon(y)}|u(y, z)|^{2} d z .
$$

Therefore,

$$
a(u, u) \geq c \int_{\Omega} H(y)^{-4}|u(x)|^{2} d x .
$$


The embedding operator $\gamma: \mathbb{H} \rightarrow L_{2}(\Omega)$ can be represented as the sum $\gamma_{0}+\gamma_{\rho}$, where $\rho \geq R$ is large and positive, $\gamma_{0}=\gamma-\gamma_{\rho}$, and $\gamma_{\rho}$ contains the operator of multiplication by the characteristic function of $\Pi_{\rho}$. The operator $\gamma_{0}$ is compact, and the norm of $\gamma_{\rho}$, in view of (14), does not exceed $c \max \left\{H\left(x_{1}\right)^{-2} ; x_{1} \geq \rho\right\}$. Since the function $H$ decays, this quantity goes to zero when $\rho \rightarrow+\infty$, i.e., the operator $\gamma$ can be approximated by compact operators in the operator norm. Thus $\gamma$ is compact and the result is proved.

\section{Auxiliary inequalities}

First of all we prove some one-dimensional weighted inequalities, two of which are of Hardy type involving a weight function $h$ as follows.

Assumption 6. Let h be a positive weight function of class $C^{2}$ on $[0,+\infty)$ such that

$\cdot \int_{0}^{\infty} h(s) d s<\infty$ and

- for some large $T$, we have $h^{\prime}(t)<0$ and $h^{\prime \prime}(t)>0$ for $t \in(T, \infty)$.

Throughout this section $h$ is supposed to satisfy this assumption.

Lemma 7. If $U$ is differentiable for $y \geq R$ and $U(R)=0$, then

$$
\int_{R}^{+\infty} h(y)|U(y)|^{2} d y \leq \int_{R}^{+\infty} F_{h}(y)\left|\partial_{y} U(y)\right|^{2} d y
$$

where

$$
F_{h}(y)=\frac{4}{h(y)}\left(\int_{y}^{+\infty} h(\tau) d \tau\right)^{2} .
$$

Proof. Using the Cauchy-Bunyakovsky-Schwarz inequality, we have

$$
\begin{aligned}
& \int_{R}^{+\infty} h(y)|U(y)|^{2} d y \\
& \quad=2 \int_{R}^{+\infty} h(y) \int_{R}^{y} \partial_{t} U(t) U(t) d t d y \\
& \quad \leq 2 \int_{R}^{+\infty} \int_{t}^{+\infty} h(y)\left|\partial_{t} U(t) U(t)\right| d y d t \\
& \quad \leq 2\left(\int_{R}^{+\infty} h(t)|U(t)|^{2} d t\right)^{1 / 2}\left(\int_{R}^{+\infty} h(t)^{-1}\left(\int_{t}^{+\infty} h(y) d y\right)^{2}\left|\partial_{t} U(t)\right|^{2} d t\right)^{1 / 2},
\end{aligned}
$$

and the result follows through division by a common factor. 
Lemma 8. If $U$ is differentiable for $y \geq R$ and $U(R)=0$, then

$$
\int_{R}^{+\infty} h(y)\left|\partial_{y} U(y)\right|^{2} d y \geq \int_{R}^{+\infty} G_{h}(y)|U(y)|^{2} d y,
$$

where

$$
G_{h, R}(y)=\frac{1}{4 h(y)}\left(\int_{R}^{y} h(\tau)^{-1} d \tau\right)^{-2} .
$$

Proof. For functions $v$ with $v(0)=0$ the Hardy inequality tells us that

$$
\int_{0}^{+\infty} t^{-2}|v(t)|^{2} d t \leq 4 \int_{0}^{+\infty}\left|\partial_{t} v(t)\right|^{2} d t
$$

We make the change $t \mapsto y \in[R,+\infty)$ where $t=\int_{R}^{y} h(\tau)^{-1} d \tau$, and set $U(y)=$ $v(t)$. Then $\partial_{t} v(t)=h(y) \partial_{y} U(y)$ leads to the desired estimate.

Corollary 9. If the function $U$ is twice differentiable for $y \geq R$ and $U(R)=$ $U^{\prime}(R)=0$, then

$$
\int_{R}^{+\infty} h(t)\left|\partial_{t}^{2} U(t)\right|^{2} d t \geq W_{h}(R) \int_{R}^{+\infty} h(t)|U(t)|^{2} d t
$$

is valid with

$$
\begin{aligned}
W_{h}(R) & :=\inf _{t \in[R,+\infty)} \frac{G_{h, R}(t)}{F_{h}(t)} \\
& =\inf _{t \in[R,+\infty)} \frac{1}{16}\left(\int_{R}^{t} h(\tau)^{-1} d \tau\right)^{-2}\left(\int_{t}^{\infty} h(\tau) d \tau\right)^{-2} .
\end{aligned}
$$

Lemma 10. We have

$$
\inf _{t \in[R,+\infty)} \frac{G_{h^{3}, R}(t)}{h(t) h^{\prime}(t)^{2}} \geq 1
$$

Suppose moreover that

$$
\lim _{t \rightarrow+\infty} \partial_{t}(\log h(t))=-\infty
$$

Then

$$
W_{h}(R) \rightarrow+\infty \quad \text { and } \quad W_{h^{3}}(R) \rightarrow+\infty \quad \text { for } R \rightarrow \infty .
$$

Proof. Since $-h^{\prime}(\tau) \geq-h^{\prime}(t)>0$ for $\tau<t$, we find

$$
\begin{aligned}
\frac{G_{h^{3}, R}(t)}{h(t) h^{\prime}(t)^{2}} & =\frac{1}{4 h^{\prime}(t)^{2} h(t)^{4}}\left(\int_{R}^{t} h(\tau)^{-3} d \tau\right)^{-2} \geq \frac{1}{4 h(t)^{4}}\left(-\int_{R}^{t} h^{\prime}(\tau) h(\tau)^{-3} d \tau\right)^{-2} \\
& =\frac{1}{4 h(t)^{4}}\left(\frac{1}{2 h(t)^{2}}-\frac{1}{2 h(R)^{2}}\right)^{-2} \geq 1
\end{aligned}
$$


Since (18) equals $h^{\prime}(t) / h(t) \rightarrow-\infty$ for $t \rightarrow \infty$, we find that for $t \rightarrow \infty$ both

$$
h(t)\left(\int_{t}^{\infty} h(\tau) d \tau\right)^{-1} \rightarrow \infty \quad \text { and } \quad \frac{1}{h(t)}\left(\int_{R}^{t} \frac{1}{h(\tau)} d \tau\right)^{-1} \rightarrow \infty .
$$

Hence

$$
\frac{G_{h, R}(t)}{F_{h}(t)} \rightarrow \infty \quad \text { as } t \rightarrow \infty,
$$

and since the quotient also goes to infinity for $t \downarrow R$, it has a minimum in some $t_{R} \in(R, \infty)$. Calculating $\left(G_{h, R}(t) / F_{h}(t)\right)^{\prime}=0$ we find

$$
\frac{1}{h(t)} \int_{t}^{\infty} h(\tau) d \tau-h(t) \int_{R}^{t} h(\tau)^{-1} d \tau=0 .
$$

Hence

$$
\inf _{t \in[R, \infty)}\left(\int_{R}^{t} h(\tau)^{-1} d \tau \int_{t}^{\infty} h(\tau) d \tau\right)^{-1}=h\left(t_{R}\right)^{2}\left(\int_{t_{R}}^{\infty} h(\tau) d \tau\right)^{-2},
$$

which goes to infinity for $R \rightarrow \infty$ since $t_{R}>R$. The claim for $W_{h}(R)$ follows. The same argument holds true for $W_{h^{3}}(R)$.

\section{Estimates for a traction-free boundary}

We assume that the Neumann boundary conditions (6) are imposed at the both sides of the peak (1). Let us describe for $\rho \rightarrow+\infty$ the behavior of the multiplier $K(\rho)$ in the inequality

$$
K(\rho) \int_{\Pi_{\rho}}|u(y, z)|^{2} d y \leq\left\|u ; H^{2}(\Omega)\right\|^{2}, \quad u \in H^{2}(\Omega) .
$$

If $K(\rho)$ increases unboundedly as $\rho \rightarrow+\infty$ then, as above, Theorem 10.1.5 of [Birman and Solomyak 1980] ensures that the spectrum of the equation (4)-(7) stays discrete even in the case both sides of the peak are supplied with the tractionfree boundary conditions $(\mathrm{N})$ and, moreover, for any other boundary conditions from the list (5)-(7).

Proposition 11. Suppose that Assumption 6 is satisfied for $h=H$ and that

$$
\lim _{t \rightarrow \infty} \partial_{t}(\log H(t))=-\infty .
$$

Then for $\rho$ sufficiently large (19) holds true with

$$
K(\rho)=c \min \left\{H^{-4}(\rho), W_{H}(\rho), W_{H^{3}}(\rho)\right\} .
$$


Proof. It is sufficient to check the inequality (19) for smooth functions which vanish for $y<\rho$. We use the representation

$$
u(x)=u(y, z)=u_{0}(y)+z u_{1}(y)+u^{\perp}(y, z)
$$

where, for $y>\rho$, the component $u^{\perp}$ is subject to the orthogonality conditions

$$
\begin{aligned}
& \int_{\Upsilon(y)} u^{\perp}(y, z) d z=0, \\
& \int_{\Upsilon(y)} \partial_{z} u^{\perp}(y, z) d z=u^{\perp}(y, H(y))-u^{\perp}(y,-H(y))=0 .
\end{aligned}
$$

Let us process the integrals on the right-hand side of

$$
\int_{\Pi_{\rho}}\left|\nabla_{x}^{2} u(x)\right|^{2} d x=I_{1}+4 I_{2}+I_{3}
$$

where

$$
I_{1}:=\int_{\Pi_{\rho}}\left|\partial_{z}^{2} u(x)\right|^{2} d x, \quad I_{2}:=\int_{\Pi_{\rho}}\left|\partial_{y} \partial_{z} u(x)\right|^{2} d x, \quad I_{3}:=\int_{\Pi_{\rho}}\left|\partial_{y}^{2} u(x)\right|^{2} d x .
$$

Since $I_{1}=\int_{\rho}^{+\infty} \int_{\Upsilon(y)}\left|\partial_{z}^{2} u^{\perp}(y, z)\right|^{2} d z d y$ and since, by the orthogonality conditions in (21), inequality (13) holds here also, we find that

$$
I_{1} \geq c \int_{\Pi_{\rho}} H(y)^{-4}\left|u^{\perp}(x)\right|^{2} d x .
$$

For the last term in (22) we have

$$
I_{3}=\int_{\Pi_{\rho}}\left|\partial_{y}^{2} u_{0}(y)+z \partial_{y}^{2} u_{1}(y)+\partial_{y}^{2} u^{\perp}(y, z)\right|^{2} d x \geq J_{1}+J_{2}+2 J_{3}+2 J_{4},
$$

where

$$
\begin{array}{ll}
J_{1}=g \int_{\Pi_{\rho}}\left|\partial_{y}^{2} u_{0}(y)\right|^{2} d x, & J_{3}=g \int_{\Pi_{\rho}} \partial_{y}^{2} u_{0}(y) \partial_{y}^{2} u^{\perp}(y, z) d x \\
J_{2}=g \int_{\Pi_{\rho}}\left|z \partial_{y}^{2} u_{1}(y)\right|^{2} d x, & J_{4}=g \int_{\Pi_{\rho}} z \partial_{y}^{2} u_{1}(y) \partial_{y}^{2} u^{\perp}(y, z) d x
\end{array}
$$

We readily notice that according to the inequality (15) the estimates

$$
J_{1} \geq W_{H}(\rho) \int_{\rho}^{+\infty} 2 H(y)\left|u_{0}(y)\right|^{2} d y=W_{H}(\rho) \int_{\Pi_{\rho}}\left|u_{0}(y)\right|^{2} d x
$$


and

$$
\begin{aligned}
J_{2} & =\frac{2}{3} \int_{\rho}^{+\infty} H^{3}(y)\left|\partial_{y}^{2} u_{1}(y)\right|^{2} d y \geq \frac{2}{3} W_{H^{3}}(\rho) \int_{\rho}^{+\infty} H^{3}(y)\left|u_{1}(y)\right|^{2} d y \\
& =W_{H^{3}}(\rho) \int_{\Pi_{\rho}}\left|z u_{1}(y)\right|^{2} d x
\end{aligned}
$$

are fulfilled. For our purpose we need $W_{H}(\rho) \rightarrow+\infty$ and $W_{H^{3}}(\rho) \rightarrow+\infty$ for $\rho \rightarrow \infty$ and this we will assume.

Besides, by the Cauchy-Bunyakovsky-Schwarz inequality, we have

$$
\left|J_{3}\right| \leq J_{1}^{1 / 2}\left(\int_{\rho}^{+\infty} \frac{1}{2 H(y)}\left(\int_{\Upsilon(y)} \partial_{y}^{2} u^{\perp}(y, z) d z\right)^{2} d y\right)^{1 / 2} .
$$

We now deal with the inner integral in $z$ in the last expression. To this end, we take into account the orthogonality conditions (21) and the trace inequality. We differentiate the first equality in (21) twice with respect to $y$ and obtain

$$
\begin{array}{r}
\sum_{ \pm}\left(2 \partial_{y} u^{\perp}(y, \pm H(y)) \partial_{y} H(y)+u^{\perp}(y, \pm H(y)) \partial_{y}^{2} H(y) \pm \partial_{z} u^{\perp}(y, \pm H(y))\left(\partial_{y} H(y)\right)^{2}\right) \\
+\int_{\Upsilon(y)} \partial_{y}^{2} u^{\perp}(y, z) d z=0 .
\end{array}
$$

Thus,

$$
\begin{aligned}
& \left(\int_{\Upsilon(y)} \partial_{y}^{2} u^{\perp}(y, z) d z\right)^{2} \\
& \quad \leq c \sum_{ \pm}\left(\left|\partial_{z} u^{\perp}(y, \pm H(y))\right|^{2}\left|\partial_{y} H(y)\right|^{4}+\left|u^{\perp}(y, \pm H(y))\right|^{2}\left|\partial_{y}^{2} H(y)\right|^{2}\right. \\
& \left.+\left|\partial_{y} u^{\perp}(y, \pm H(y))\right|^{2}\left|\partial_{y} H(y)\right|^{2}\right) .
\end{aligned}
$$

For the first two terms between the brackets we use the trace inequality

$$
\left|\partial_{z} u^{\perp}(y, \pm H(y))\right|^{2}|H(y)|^{2}+\left|u^{\perp}(y, \pm H(y))\right|^{2} \leq c|H(y)|^{3} \int_{\Upsilon(y)}\left|\partial_{z}^{2} u^{\perp}(y, z)\right|^{2} d z .
$$

For the third term, we write down the chain of inequalities

$$
\begin{aligned}
& \left|\partial_{y} u^{\perp}(y, \pm H(y))\right|^{2} \\
& \leq c H(y) \int_{\Upsilon(y)}\left|\partial_{y} \partial_{z} u^{\perp}(y, z)\right|^{2} d z+c|H(y)|^{-2}\left(\int_{\Upsilon(y)} \partial_{y} u^{\perp}(y, z) d z\right)^{2} \\
& \leq c H(y) \int_{\Upsilon(y)}\left|\partial_{y} \partial_{z} u^{\perp}(y, z)\right|^{2} d z \\
& \quad+2 c\left(\frac{\partial_{y} H(y)}{H(y)}\right)^{2}\left(\left|u^{\perp}(y, H(y))\right|^{2}+\left|u^{\perp}(y,-H(y))\right|^{2}\right) .
\end{aligned}
$$


As a result, we find that

$$
\begin{array}{r}
\left|\int_{\Upsilon(y)} \partial_{y}^{2} u^{\perp}(y, z) d z\right|^{2} \leq c\left(\left|\partial_{y} H(y)\right|^{4} H(y)+\left|\partial_{y}^{2} H(y)\right|^{2}|H(y)|^{3}\right) \int_{\Upsilon(y)}\left|\partial_{z}^{2} u^{\perp}(y, z)\right|^{2} d z \\
+c\left|\partial_{y} H(y)\right|^{2}|H(y)| \int_{\Upsilon(y)}\left|\partial_{y} \partial_{z} u^{\perp}(y, z)\right|^{2} d z .
\end{array}
$$

The final inequality for the integral $J_{3}$ takes the form

$$
\left|J_{3}\right| \leq c_{1}(\rho) J_{1}^{1 / 2} I_{1}^{1 / 2}+c_{2}(\rho) J_{1}^{1 / 2} K_{1}^{1 / 2}
$$

where $K_{1}=\left\|\partial_{y z}^{2} u^{\perp} ; L_{2}\left(\Pi_{\rho}\right)\right\|^{2}$ and

$$
\begin{aligned}
& c_{1}(\rho)=c \sup _{y \in[\rho,+\infty)}\left(\left|\partial_{y} H(y)\right|^{2}+\left|\partial_{y}^{2} H(y)\right||H(y)|\right), \\
& c_{2}(\rho)=c \sup _{y \in[\rho,+\infty)}\left|\partial_{y} H(y)\right| .
\end{aligned}
$$

Both suprema tend to 0 for $\rho \rightarrow+\infty$. A similar argument shows that

$$
\left|J_{4}\right| \leq c_{1}(\rho) J_{2}^{1 / 2} I_{1}^{1 / 2}+c_{2}(\rho) J_{2}^{1 / 2} K_{1}^{1 / 2} .
$$

It remains to process the second term in (22), that is,

$$
\begin{aligned}
I_{2} & =\int_{\Pi_{\rho}}\left|z \partial_{y} u_{1}(y)+\partial_{y} \partial_{z} u^{\perp}(y, z)\right|^{2} d x \\
& =\int_{\Pi_{\rho}}\left|\partial_{y} \partial_{z} u^{\perp}(y, z)\right|^{2} d x+\int_{\Pi_{\rho}}\left|\partial_{y} u_{1}(y)\right|^{2} d x+2 \int_{\Pi_{\rho}} \partial_{y} u_{1}(y) \partial_{y} \partial_{z} u^{\perp}(y, z) d x . \\
K_{1} & \aleph_{K_{2}}
\end{aligned}
$$

So it follows that

$$
K_{1}=I_{2}-K_{2}-2 K_{3} \leq I_{2}+2\left|K_{3}\right| .
$$

We continue by estimating the integral $K_{3}$ :

$$
\begin{aligned}
& \left|K_{3}\right|=\left|\int_{\rho}^{+\infty} \partial_{y} u_{1}(y) \int_{\Upsilon(y)} \partial_{y} \partial_{z} u^{\perp}(y, z) d z d y\right| \\
& \leq\left(\int_{\rho}^{+\infty} G_{H^{3}, \rho}(y)\left|\partial_{y} u_{1}(y)\right|^{2} d y\right)^{\frac{1}{2}}\left(\int_{\rho}^{+\infty} G_{H^{3}, \rho}(y)^{-1}\left|\int_{\Upsilon(y)} \partial_{y} \partial_{z} u^{\perp}(y, z) d z\right|^{2} d y\right)^{\frac{1}{2}} \\
& \leq c J_{2}^{1 / 2}\left(\int_{\rho}^{+\infty} G_{H^{3}, \rho}(y)^{-1}\left|\int_{\Upsilon(y)} \partial_{y} \partial_{z} u^{\perp}(y, z) d z\right|^{2} d y\right)^{1 / 2} .
\end{aligned}
$$

Differentiating the second formula (21) with respect to $y$ yields

$$
\int_{\Upsilon(y)} \partial_{y} \partial_{z} u^{\perp}(y, z) d z+\sum_{ \pm} \partial_{z} u^{\perp}(y, \pm H(y)) \partial_{y} H(y)=0 .
$$


By the trace inequality we find that

$$
\begin{aligned}
\left|\int_{\Upsilon(y)} \partial_{y} \partial_{z} u^{\perp}(y, z) d z\right|^{2} & =\left(\sum_{ \pm} \partial_{z} u^{\perp}(y, \pm H(y))\right)^{2}\left|\partial_{y} H(y)\right|^{2} \\
& \leq c\left|H(y) \| \partial_{y} H(y)\right|^{2} \int_{\Upsilon(y)}\left|\partial_{z}^{2} u^{\perp}(y, z)\right|^{2} d z .
\end{aligned}
$$

Thus, from the relation (18), which implies (17), we get

$$
\left|K_{3}\right| \leq c \sup _{y \in[\rho,+\infty)}\left\{\left|G_{H^{3}, \rho}(y)\right|^{-1 / 2}\left|\partial_{y} H(y)\right||H(y)|^{1 / 2}\right\} J_{2}^{1 / 2} I_{1}^{1 / 2} \leq c J_{2}^{1 / 2} I_{1}^{1 / 2} .
$$

We find by combining (29) and (30) that

$$
K_{1} \leq I_{2}+c J_{2}^{1 / 2} I_{1}^{1 / 2}
$$

and so (27) and (28) yield, respectively,

$$
\begin{aligned}
& \left|J_{3}\right| \leq c_{1}(\rho) J_{1}^{1 / 2} I_{1}^{1 / 2}+c_{2}(\rho) J_{1}^{1 / 2}\left(I_{2}+c J_{2}^{1 / 2} I_{1}^{1 / 2}\right)^{1 / 2}, \\
& \left|J_{4}\right| \leq c_{1}(\rho) J_{2}^{1 / 2} I_{1}^{1 / 2}+c_{2}(\rho) J_{2}^{1 / 2}\left(I_{2}+c J_{2}^{1 / 2} I_{1}^{1 / 2}\right)^{1 / 2} .
\end{aligned}
$$

Using first (22) and (24), next (31) and (32) for $\rho$ large enough, and finally (23), (25) and (26) we conclude that indeed

$$
\begin{aligned}
\left\|\nabla_{x}^{2} u ; L_{2}\left(\Pi_{\rho}\right)\right\|^{2} & =I_{1}+4 I_{2}+I_{3} \\
& \geq I_{1}+4 I_{2}+J_{1}+J_{2}+2 J_{3}+2 J_{4} \\
& \geq \frac{1}{2}\left(I_{1}+4 I_{2}+J_{1}+J_{2}\right) \geq \frac{1}{2}\left(I_{1}+J_{1}+J_{2}\right) \\
& \geq c \min \left\{H^{-4}(\rho), W_{H}(\rho), W_{H^{3}}(\rho)\right\}\left\|u ; L_{2}\left(\Pi_{\rho}\right)\right\|^{2},
\end{aligned}
$$

whenever $\rho$ is large enough.

\section{Traction-free boundaries: $N-N$}

Theorem 12. Suppose that $H$ satisfies Assumption 6 with $h=H$ and that

$$
\lim _{t \rightarrow \infty} \partial_{t}(\log H(t))=-\infty .
$$

Then the embedding $H^{2}(\Omega) \hookrightarrow L_{2}(\Omega)$ is compact and the spectrum of the problem (4) with the Neumann boundary conditions (6) on both sides of the peak is discrete. Proof. By Proposition 11, $K(\rho)$ can be estimated as in (20). Assumption 6 implies that Lemma 10 holds true and hence $K(\rho) \rightarrow+\infty$ for $\rho \rightarrow+\infty$. One concludes as in the proof of Theorem 5 through an approximation by compact operators. 
Remark 12.1. Note that the functions $H(y)=y^{-\alpha}$ and $H(y)=\exp (-\alpha y), \alpha>0$, do not satisfy the requirement in (2) or (33). The functions $H(y)=\exp \left(-y^{1+\alpha}\right)$ with $\alpha>0$ however do.

\section{An incomplete Dirichlet condition: $M-N$}

In this section the boundary conditions only contain a single stable condition

$$
u(x)=0, \quad x \in \Sigma_{\rho}^{+}\left(\text {or } x \in \Sigma_{\rho}^{-}\right) .
$$

Theorem 13. Suppose that $\lim _{y \rightarrow \infty} H(y)^{-3} G_{H}(y)=+\infty$. Then the problem in (4) with the boundary condition as in (34) has discrete spectrum.

Remark 13.1. The functions $H(y)=y^{-1-\alpha}$ with $\alpha>0$ meet the condition in Theorem 13.

Proof. By (34), Friedrich's inequality holds on the section $\Upsilon(y)$ and, consequently,

$$
\left\|\mathscr{A} \partial_{z} u ; L_{2}\left(\Pi_{\rho}\right)\right\|^{2} \geq c\left\|\mathscr{A} H^{-2} u ; L_{2}\left(\Pi_{\rho}\right)\right\|^{2}
$$

for every positive weight function $y \mapsto \mathscr{A}(y)$. The function $v=\partial_{z} u$ can be represented as the sum $v(x)=v_{0}(y)+v^{\perp}(x)$ where, for $y>\rho$, the component $v^{\perp}$ satisfies the first condition in (21). Therefore,

$$
\begin{aligned}
& \int_{\Pi_{\rho}}\left|\nabla_{x}^{2} u(x)\right|^{2} d x \\
& \geq \int_{\Pi_{\rho}}\left|\nabla_{x} v(x)\right|^{2} d x \\
& \geq \int_{\rho}^{+\infty} 2 H(y)\left|\partial_{y} v_{0}(y)\right|^{2} d y+\int_{\Pi_{\rho}}\left|\partial_{z} v^{\perp}(y, z)\right|^{2} d x+2 \int_{\Pi_{\rho}} \partial_{y} v_{0}(y) \partial_{y} v^{\perp}(y, z) d x \\
& =: I_{4}+I_{5}+2 I_{6} .
\end{aligned}
$$

Setting $Z_{H}(y)=H(y)^{-1} G_{H}(y)$, we get

$$
\begin{aligned}
I_{4} \geq \int_{\rho}^{+\infty} 2 G_{H}(y)\left|v_{0}(y)\right|^{2} d y & \geq \int_{\rho}^{+\infty} 2 Z_{H}(y) H(y)\left|v_{0}(y)\right|^{2} d y \\
& =\left\|Z_{H} v_{0} ; L_{2}\left(\Pi_{\rho}\right)\right\|^{2} .
\end{aligned}
$$

Friedrich's inequality implies

$$
I_{5}=\left\|\partial_{z} v^{\perp} ; L_{2}\left(\Pi_{\rho}\right)\right\| \geq c\left\|H^{-2} v^{\perp} ; L_{2}\left(\Pi_{\rho}\right)\right\|^{2} .
$$


Furthermore,

$$
\begin{aligned}
I_{6} & =\int_{\Pi_{\rho}} \partial_{y} v_{0}(y) \partial_{y} v^{\perp}(y, z) d x \leq I_{5}^{1 / 2}\left(\int_{\rho}^{+\infty} \frac{1}{2 H(y)}\left|\int_{\Upsilon(y)} \partial_{y} v^{\perp}(y, z) d z\right|^{2}\right)^{1 / 2} \\
& \leq I_{5}^{1 / 2}\left(\int_{\rho}^{+\infty} \frac{1}{2 H(y)}\left|v^{\perp}(y, H(y)) \partial_{y} H(y)-v^{\perp}(y,-H(y)) \partial_{y} H(y)\right|^{2}\right)^{1 / 2} .
\end{aligned}
$$

Thus $I_{6} \leq c\left|\partial_{y} H(\rho)\right| I_{5}^{1 / 2} I_{6}^{1 / 2}$ holds and hence

$$
\left\|\nabla_{x}^{2} u ; L_{2}\left(\Pi_{\rho}\right)\right\|^{2} \geq c\left\|\min \left\{H^{-4} ; H^{-3} G_{H}\right\} u ; L_{2}\left(\Pi_{\rho}\right)\right\|^{2} .
$$

Compactness and hence the discrete spectrum follow from the assumption on $H$.

\section{References}

[Adams and Fournier 2003] R. A. Adams and J. J. F. Fournier, Sobolev spaces, 2nd ed., Pure and Applied Mathematics 140, Elsevier/Academic Press, Amsterdam, 2003.

[Bakharev and Nazarov 2009] F. L. Bakharev and S. A. Nazarov, "On the structure of the spectrum of a problem in the theory of elasticity for a body with a supersharp peak", Sibirsk. Mat. Zh. 50:4 (2009), 746-756. In Russian; translated in Sib. Math. J. 50:4 (2009), 587-595.

[van den Berg 1984] M. van den Berg, "On the spectrum of the Dirichlet Laplacian for horn-shaped regions in $\mathbf{R}^{n}$ with infinite volume”, J. Funct. Anal. 58:2 (1984), 150-156.

[van den Berg and Lianantonakis 2001] M. van den Berg and M. Lianantonakis, "Asymptotics for the spectrum of the Dirichlet Laplacian on horn-shaped regions", Indiana Univ. Math. J. 50:1 (2001), 299-333.

[Birman and Solomyak 1980] M. S. Birman and M. Z. Solomyak, Спектралная теория самосопряженных операторов в гилбертовом пространстве, Leningrad Univ., 1980. Translated as Spectral theory of selfadjoint operators in Hilbert space, Reidel, Dordrecht, 1987.

[Boyarchenko and Levendorskii 2000] S. I. Boyarchenko and S. Z. Levendorskii, "Spectral asymptotics of the Neumann Laplacian on oscillating horns", Russ. J. Math. Phys. 7:1 (2000), 1-14.

[Courant and Hilbert 1953] R. Courant and D. Hilbert, Methods of mathematical physics, vol. 1, Interscience, New York, 1953.

[Davies and Simon 1992] E. B. Davies and B. Simon, "Spectral properties of Neumann Laplacian of horns", Geom. Funct. Anal. 2:1 (1992), 105-117.

[Evans and Harris 1987] W. D. Evans and D. J. Harris, "Sobolev embeddings for generalized ridged domains", Proc. London Math. Soc. (3) 54:1 (1987), 141-175.

[Gazzola et al. 2010] F. Gazzola, H.-C. Grunau, and G. Sweers, Polyharmonic boundary value problems: Positivity preserving and nonlinear higher order elliptic equations in bounded domains, Lecture Notes in Mathematics 1991, Springer, Berlin, 2010.

[Hempel et al. 1991] R. Hempel, L. A. Seco, and B. Simon, "The essential spectrum of Neumann Laplacians on some bounded singular domains", J. Funct. Anal. 102:2 (1991), 448-483.

[Ivrii 1999] V. Ivrii, "Precise spectral asymptotics for Neumann Laplacian in domains with cusps", Appl. Anal. 71:1-4 (1999), 139-147.

[Jakšić 1993] V. Jakšić, "On the spectrum of the Neumann Laplacian of long-range horns: a note on the Davies-Simon theorem”, Proc. Amer. Math. Soc. 119:2 (1993), 663-669. 
[Jakšić et al. 1992] V. Jakšić, S. Molčanov, and B. Simon, "Eigenvalue asymptotics of the Neumann Laplacian of regions and manifolds with cusps", J. Funct. Anal. 106:1 (1992), 59-79.

[Kovařík 2011] H. Kovařík, "Eigenvalue asymptotic of Robin Laplace operators on two-dimensional domains with cusps", J. Lond. Math. Soc. (2) 83:1 (2011), 256-271.

[Krylov and Tilman 2004] V. V. Krylov and F. J. B. S. Tilman, "Acoustic 'black holes' for flexural waves as effective vibration dampers", J. Sound Vib. 274 (2004), 605-619.

[Maz'ya and Poborchi 1997] V. G. Maz'ya and S. V. Poborchi, Differentiable functions on bad domains, World Scientific, River Edge, NJ, 1997.

[Maz'ya and Poborchii 2006] V. G. Maz'ya and S. V. Poborchii, “Theorems for embedding Sobolev spaces on domains with a peak and on Hölder domains", Algebra i Analiz 18:4 (2006), 95-126.

[Mikhlin 1970] S. G. Mikhlin, Вариационные методы в математической физике, 2nd ed., Nauka, Moscow, 1970. First edition translated as Variational methods in mathematical physics, Pergamon Press, Oxford, 1964.

[Mironov 1988] M. A. Mironov, "Propagation of a flexural wave in a plate whose thickness decreases smoothly to zero in a finite interval”, Soviet Phys. Acoust. 34 (1988), 318-319.

[Nazarov 1999] S. A. Nazarov, "Polynomial property of selfadjoint elliptic boundary value problems, and the algebraic description of their attributes", Uspekhi Mat. Nauk 54:5(329) (1999), 77142. In Russian; translated in Russian Math. Surveys 54:5 (1999), 947-1014.

[Nazarov 2002] S. A. Nazarov, Asymptotic theory of thin plates and rods, vol. 1: Dimension reduction and integral estimates, Nauchnaya Kniga, Novosibirsk, 2002.

[Nazarov 2008] S. A. Nazarov, "On the spectrum of a problem in the theory of elasticity for a spiked body”, Sibirsk. Mat. Zh. 49:5 (2008), 1105-1127.

[Nazarov 2009] S. A. Nazarov, "On the essential spectrum of boundary value problems for systems of differential equations in a bounded domain with a cusp", Funktsional. Anal. i Prilozhen. 43:1 (2009), 55-67. In Russian; translated in Funct. Anal. Appl. 43:1 (2009), 44-54.

[Nazarov and Taskinen 2008] S. A. Nazarov and J. Taskinen, "On the spectrum of the Steklov problem in a domain with a peak", Vestnik Sankt-Pet. Univ. Ser. 1 Mat. Mekh. Astr. 2008:1 (2008), 56-65. In Russian; translated in Vestnik St. Petersburg Univ. Math. 41:1 (2008), 45-52.

[Rellich 1948] F. Rellich, "Das Eigenwertproblem von $\Delta u+\lambda u=0$ in Halbröhren", pp. 329-344 in Studies and essays presented to R. Courant on his 60th birthday, Interscience, New York, 1948.

[Rozenbljum 1972] G. V. Rozenbljum, "The eigenvalues of the first boundary value problem in unbounded domains", Mat. Sb. (N.S.) 89:(131) (1972), 234-247. In Russian; translated in Math. USSR Sb. 18 (1972), 235-248 (1973).

[Simon 1992] B. Simon, “The Neumann Laplacian of a jelly roll”, Proc. Amer. Math. Soc. 114:3 (1992), 783-785.

Received 7 Mar 2012. Revised 21 Jul 2012. Accepted 28 Aug 2012.

FEDOR L. BAKHAREV: fbakharev@yandex.ru

Mathematics and Mechanics Faculty, St. Petersburg State University, 198904 St. Petersburg, Russia

SERGEY A. NAZARov: srgnazarov@yahoo.co.uk

Institute of Problems of Mechanical Engineering, Russian Academy of Sciences,

199178 St. Petersburg, Russia

Current address: Mathematics and Mechanics Faculty, St. Petersburg State University,

Universitetsky pr., 28, 198504, Stary Peterhof, Russia

GUIDO H. SWEERS: gsweers@math.uni-koeln.de

Mathematisches Institut, Universität zu Köln, D-50931 Cologne, Germany

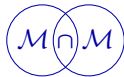




\section{Guidelines for Authors}

Authors may submit manuscripts in PDF format on-line at the submission page.

Originality. Submission of a manuscript acknowledges that the manuscript is original and and is not, in whole or in part, published or under consideration for publication elsewhere. It is understood also that the manuscript will not be submitted elsewhere while under consideration for publication in this journal.

Language. Articles in MEMOCS are usually in English, but articles written in other languages are welcome.

Required items. A brief abstract of about 150 words or less must be included. It should be selfcontained and not make any reference to the bibliography. If the article is not in English, two versions of the abstract must be included, one in the language of the article and one in English. Also required are keywords and a Mathematics Subject Classification or a Physics and Astronomy Classification Scheme code for the article, and, for each author, postal address, affiliation (if appropriate), and email address if available. A home-page URL is optional.

Format. Authors are encouraged to use $\mathrm{IAT}_{\mathrm{E} X}$ and the standard amsart class, but submissions in other varieties of $\mathrm{T}_{\mathrm{E}} \mathrm{X}$, and exceptionally in other formats, are acceptable. Initial uploads should normally be in PDF format; after the refereeing process we will ask you to submit all source material.

References. Bibliographical references should be complete, including article titles and page ranges. All references in the bibliography should be cited in the text. The use of $\mathrm{BIBT}_{\mathrm{E}} \mathrm{X}$ is preferred but not required. Tags will be converted to the house format, however, for submission you may use the format of your choice. Links will be provided to all literature with known web locations and authors are encouraged to provide their own links in addition to those supplied in the editorial process.

Figures. Figures must be of publication quality. After acceptance, you will need to submit the original source files in vector graphics format for all diagrams in your manuscript: vector EPS or vector PDF files are the most useful.

Most drawing and graphing packages - Mathematica, Adobe Illustrator, Corel Draw, MATLAB, etc. - allow the user to save files in one of these formats. Make sure that what you are saving is vector graphics and not a bitmap. If you need help, please write to graphics@msp.org with as many details as you can about how your graphics were generated.

Bundle your figure files into a single archive (using zip, tar, rar or other format of your choice) and upload on the link you been provided at acceptance time. Each figure should be captioned and numbered so that it can float. Small figures occupying no more than three lines of vertical space can be kept in the text ("the curve looks like this:"). It is acceptable to submit a manuscript with all figures at the end, if their placement is specified in the text by means of comments such as "Place Figure 1 here". The same considerations apply to tables.

White Space. Forced line breaks or page breaks should not be inserted in the document. There is no point in your trying to optimize line and page breaks in the original manuscript. The manuscript will be reformatted to use the journal's preferred fonts and layout.

Proofs. Page proofs will be made available to authors (or to the designated corresponding author) at a Web site in PDF format. Failure to acknowledge the receipt of proofs or to return corrections within the requested deadline may cause publication to be postponed. 
Mathematics and Mechanics of Complex Systems vol. 1 no. 2

Self-organized stochastic tipping in slow-fast dynamical systems

Mathias Linkerhand and Claudius Gros

Well-posedness for dislocation-based gradient

viscoplasticity, II: general nonassociative monotone plastic flows

Sergiy Nesenenko and Patrizio Neff

Symmetry classes for even-order tensors

Marc Olive and Nicolas Auffray

A direct approach to nonlinear shells with application to surface-substrate interactions

Miroslav Šilhavý

A sufficient condition for a discrete spectrum of the Kirchhoff plate with an infinite peak

Fedor L. Bakharev, Sergey A. Nazarov and Guido H. Sweers

MEMOCS is a journal of the International Research Center for the Mathematics and Mechanics of Complex Systems at the Università dell' Aquila, Italy.

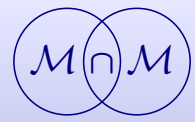

